\title{
Modelling the Melting of Gallium Clusters: A Path to Understanding Molecular Solids
}

\author{
by \\ Krista Grace Steenbergen
}

\author{
A thesis \\ submitted to the Victoria University of Wellington \\ in fulfilment of the \\ requirements for the degree of \\ Doctor of Philosophy \\ in Physics.
}

Victoria University of Wellington 2013 



\begin{abstract}
Gallium is a molecular solid with many unique properties. Comprised of $\mathrm{Ga}_{2}$ dimers but exhibiting metal-like electronic characteristics, gallium may be deemed a molecular metal. The role of this dual covalent-metallic nature may explain gallium's fascinating thermodynamic behaviour. While bulk gallium melts at $303 \mathrm{~K}$, clusters with only 10's of atoms melt at temperatures between 500 and $800 \mathrm{~K}$, according to experiment. The measured specific heat curves exhibit a strong size-sensitivity, where the addition of a single atom can alter the melting temperature by up to $100 \mathrm{~K}$. This research addresses the relationship of electronic structure to the melting behaviour in small gallium clusters through a parallel tempering implementation of first-principles molecular dynamics simulations. These simulations cover 11 cluster sizes and two charge states, including neutral clusters sized 7-12 atoms and cationic clusters sized 32-35 atoms. The modelling of small clusters sets a lower size limit for melting and illustrates that greater-than-bulk melting is not universal for small gallium clusters. The larger cluster sizes allow for a direct comparison to experimental data. Each simulation reveals that the clusters have a non-covalent nature more similar to the metallic surface structure of bulk gallium than its covalently bonded interior. The dramatic lowering of melting temperatures and cluster stabilities with single atom additions supports the conclusion that the difference in the nature of bonding between bulk and clusters accounts for the melting temperature discrepancy. Finally, in order to gain additional insight into the nature of bonding in molecular solids, the cohesive energies of the solid halogens are calculated by the method of increments. These calculations investigate the relative N-body correlation energy contributions to the total cohesive energy for solid $\mathrm{Cl}_{2}, \mathrm{Br}_{2}$ and $\mathrm{I}_{2}$.
\end{abstract}




\section{Acknowledgments}

To my friends and colleagues here in New Zealand ... Shrividya, Dion, Dmitri, Amanda, Nat, Laurie, and Frenchies Francois and Gregory: Thank you for your friendship, support and laughter over these past three years.

To my wonderful friends and family in the northern hemisphere... mom, Junebug, Beth, Mags, Angie, Maiya, Dr. Bailey, Ron, Jim, Pete: Thank you for everything you've added to my life over all these years.

To Andrew ... from my decision to look into the PhD to my oral defense, you have supported me in this pursuit - adding insight, perspective and laughter to my life. You are a marvelous instructor ... and you have been such a wonderful friend. I am so very grateful to have you in my life.

To dad ... for all the math games on the way to the lake, for your enthusiasm and pride as I pursued math and science, for being the calm voice of logic and solid ground for all of my 37 years - thank you so incredibly much. You've been the best father a girl could possibly have and I love you!

To the HPC system administrators, particularly Tony Dale (BlueFern), Michael Sternberg (Argonne) and Benjamin Roberts (Auckland): Thank you for sharing your knowledge, patience and support.

To my secondary supervisors, Prof. Shaun Hendy, Prof. Beate Paulus and Dr. Carsten Müller: Thank you for your time, support, discussion and encouragement. I have learned and grown so much during this PhD. Your guidance and mentorship have been invaluable.

Finally, and most importantly, to my supervisor, Dr. Nicola Gaston: I 
have so thoroughly enjoyed working with you to explore the riddles of gallium (and other elements). Your kindness, patience, encouragement, and enthusiasm have turned an interesting research topic into a captivating adventure. Our interactions and your wonderful mentorship have helped me to grow both as a scientist and person. I have to chuckle as I'm reminded of my comment during my first presentation of this $\mathrm{PhD}$, 3 years ago at Cluster Day 2009. I was right: You truly are br. and bt.! You have made this $\mathrm{PhD}$ an exceptional experience ... and I am so very grateful. 


\section{Contents}

1 Molecular Solids 1

1.1 Gallium ....................... 2

1.1 .1 Bulk .................. 3

1.1.2 Small Clusters . . . . . . . . . . . . . . 8

1.1.3 Experimental measurements . . . . . . . . . . 11

1.1.4 Classical simulations . . . . . . . . . . . 13

1.1.5 First-principles and ab initio modelling . . . . . . . 15

1.2 Solid Halogens . . . . . . . . . . . . . . . . . . . . . 21

2 Theory 25

2.1 Born-Oppenheimer Approximation . . . . . . . . . . 27

2.2 Mathematical Background . . . . . . . . . . . . . . . . . 29

2.2.1 Antisymmetry . . . . . . . . . . . . . 30

2.2 .2 Spin Orbitals . . . . . . . . . . . . . . . 30

2.2 .3 Hartree Product . . . . . . . . . . . . . . . . . . . 31

2.2.4 Slater Determinants . . . . . . . . . . . . 33

2.3 Hartree-Fock . . . . . . . . . . . . . . . . . . . . 35

2.4 Density Functional Theory . . . . . . . . . . . . . . . . . 40

2.4.1 Born Oppenheimer Molecular Dynamics . . . . . . . 45

2.5 Method of Increments . . . . . . . . . . . . . . . . 48

3 DFT for Gallium $\quad 55$

3.1 Symmetric Octahedron . . . . . . . . . . . . . . . 55 
3.2 Basis Sets and Functionals . . . . . . . . . . . . . 57

3.3 Gallium Dimer . . . . . . . . . . . . . . . . . . . 57

3.4 N-body Increments . . . . . . . . . . . . . . . . . 59

3.5 6-atom Gallium . . . . . . . . . . . . . . 61

4 Melting $\quad 65$

4.1 Initial structures . . . . . . . . . . . . . . . 65

4.2 Classical MD . . . . . . . . . . . . . . . 67

4.3 First-Principles MD . . . . . . . . . . . . . . 70

4.3.1 Ensemble selection . . . . . . . . . . . . 70

4.3 .2 VASP Settings . . . . . . . . . . . . . . . 71

4.4 Parallel Tempering . . . . . . . . . . . . . . 73

4.4.1 VASP Swapping . . . . . . . . . . . 75

4.5 Methods of Atomic Structure Analysis . . . . . . . . . . . 78

4.5.1 Radial distribution functions . . . . . . . . . . . 78

4.5.2 Root-mean square deviation . . . . . . . . . . . . . 79

4.5.3 Mean square displacement . . . . . . . . . . . 81

4.6 Methods of Electronic Structure Analysis . . . . . . . . . . . 82

4.6.1 Bader analysis . . . . . . . . . . . . . . 82

4.6.2 Projected electronic density of states . . . . . . . . 83

4.6.3 Electron localization function . . . . . . . . . . . 84

4.7 Methods of Thermodynamic Analysis . . . . . . . . . . . . 85

$4.7 .1 \quad$ Discrete Analysis . . . . . . . . . . . . . . . . 85

4.7.2 Multiple Histogram Analysis . . . . . . . . . . . 86

5 20-atom Gallium $\quad 97$

5.1 Initial settings . . . . . . . . . . . . . 97

5.2 Atomic structure analysis . . . . . . . . . . . . . . . 99

5.3 Effect of parallel tempering . . . . . . . . . . . . . . 101

5.4 Convergence analysis . . . . . . . . . . . . . 106

5.5 Thermodynamics . . . . . . . . . . . . . . 106

5.6 Structural dynamics . . . . . . . . . . . . . . . . . . 112 
5.7 Electronic structure . . . . . . . . . . . . . . . . . . 113

5.8 Summary . . . . . . . . . . . . . . . . . . . 119

6 Microclusters 123

6.1 Background . . . . . . . . . . . . . . . . 123

6.2 Microcluster Simulations . . . . . . . . . . . . . . . . 125

6.3 Convergence . . . . . . . . . . . . . . . . 131

6.4 Thermodynamics . . . . . . . . . . . . . . . . . 131

6.5 Structural dynamics . . . . . . . . . . . . . . . . . . . 135

6.6 Electronic structure . . . . . . . . . . . . . . . . 141

6.7 Summary . . . . . . . . . . . . . . . . . 149

7 Large Gallium Clusters 153

7.1 Structures . . . . . . . . . . . . . . . . . 153

7.2 Specific Heat Comparisons . . . . . . . . . . . . . . . . . . 154

7.3 PT-derived Structures _. . . . . . . . . . . . . . . . 162

7.4 Convergence . . . . . . . . . . . . . . . . . 167

7.5 Electronic structure . . . . . . . . . . . . . . . . 168

7.6 Summary . . . . . . . . . . . . . . . 173

8 Solid Halogens $\quad 175$

8.1 DFT for Solid Halogens . . . . . . . . . . . . . . . . . . 175

8.2 MI Incremental Calculations . . . . . . . . . . . . . . . . 177

8.2.1 Crystal structure . . . . . . . . . . . . . . 178

8.2.2 Basis sets and pseudopotentials . . . . . . . . . . 181

8.2 .3 Embedding .................. 183

8.2.4 Orbital domains . . . . . . . . . . . . . . . 187

8.2.5 Coupled-cluster excitations . . . . . . . . . . . . 189

8.2.6 Incremental Calculations . . . . . . . . . . . . . 190

8.2.7 LC Model Results . . . . . . . . . . . . . . . . . . . . . 191

8.2.8 SC Model Results . . . . . . . . . . . . . . . . . . . 192

8.3 Zero-point energies . . . . . . . . . . . . . . . . 198 
8.4 Bulk Hartree-Fock Calculations . . . . . . . . . . . . . . 198

8.4.1 Dual Basis Set Scheme . . . . . . . . . . . . . 199

8.4.2 Bulk $\mathrm{Cl}_{2}$ results ............... . 201

8.5 Summary........................ 203

9 Conclusions $\quad 207$

9.1 Gallium Clusters . . . . . . . . . . . . . . . . . 207

9.2 Solid Halogens . . . . . . . . . . . . . . . . . . . 212

9.3 Molecular Solids . . . . . . . . . . . . . . . . . . 213

$\begin{array}{ll}\text { Appendices } & 215\end{array}$

A Multiple-Histogram Mathematics 217

A.1 Deriving $S_{j} \ldots \ldots \ldots \ldots \ldots \ldots \ldots \ldots$

A.2 Deriving $A_{i} \ldots \ldots \ldots \ldots \ldots$. . . . . . . . . . . . . . . . . .

B Atomic Coordinates 223

$\begin{array}{ll}\text { C Basis Sets } & 255\end{array}$ 


\section{Chapter 1}

\section{Molecular Solids}

"The underlying physical laws necessary for the mathematical theory of a large part of physics and the whole of chemistry are thus completely known, and the difficulty is only that the exact application of these equations leads to equations much too complicated to be soluble. It therefore becomes desirable that approximate practical methods of applying quantum mechanics should be developed, which can lead to an explanation of the main features of complex atomic systems without too much computation." [1]

- Paul Dirac, 1929

The laws that dictate bonding in chemical systems are incredibly complex. Given that these laws govern every part of our lives, from how our bodies function to how our universe was formed, much of chemistry and physics research is directed at gaining a better understanding of how and why atoms coalesce to form molecules and matter. The emergence and development of quantum mechanics in the 20th century has led many scientists to believe that we now possess all the tools necessary to mathematically describe all of chemistry. And yet, over the nearly 100 years since the birth of quantum theory, the chemistry underlying the various bulk structures observed for different elemental solids in the periodic table remains largely unknown. As outlined above in Dirac's quote, this limita- 
tion arises due to the incredible mathematical complexity required to exactly describe even the simplest of systems. This complexity necessitates the approximate solutions to some of the most fundamental questions in physics and chemistry, to include how and why matter bonds, as well as how that bonding affects material properties and behavior.

This work aims to further our understanding of the material properties of two classes of molecular solids: gallium and the solid halogens, chlorine, bromine and iodine. In the bulk, solid phase, each of these elements is comprised of $X_{2}$ subunits ( $\mathrm{X}=\mathrm{Ga}, \mathrm{Cl}, \mathrm{Br}$ or $\left.\mathrm{I}\right)$, as opposed to the atomic building blocks comprising most other elements in the periodic table. The nature of bonding underlying this unique molecular structure is, at present, not well-understood, which has led to questions as to its role in several unique properties exhibited by these elements. This research addresses the intriguing greater-than-bulk melting temperatures observed in small gallium clusters and the correlation energy contributions to bonding in the solid halogens. The following sections introduce both gallium and the solid halogens, providing an overview of the specific questions to be addressed in body of the thesis.

\subsection{Gallium}

Gallium is a unique element, exhibiting a wealth of anomalous properties that continue to intrigue scientists even today, more than 130 years since its discovery. The element is interesting historically in that Dmitri Mendeleev predicted its existence, using trends in the periodic table to identify gaps in the elemental progression, nearly 4 years before it was spectroscopically verified. Mendeleev called this "missing element" eka-aluminum, and even managed to predict its atomic mass and density to a surprising degree of accuracy.

Gallium has become an important element in today's technology-dominated world. A primary component of many leading semiconductor technolo- 
gies, bits of gallium are loaded into devices ranging from satellites to cell phones. Combined with arsenic or nitrogen, it serves as an integral constituent of LEDs, multi-junction photovoltaic cells or high-speed transistors [2]. Alloyed with aluminum, it can generate hydrogen from water [3], which could play an incredibly important role in the development of a hydrogen economy. The cutting-edge applications involving this interesting metal are nearly endless and lend particular impetus to understanding its electronic and structural properties. Beginning with a description of gallium in its bulk form, this section covers the many unique properties of gallium, to include the intriguing thermodynamic behavior observed in small clusters.

\subsubsection{Bulk}

Although periodic trends in the known elements led Mendeleev to predict gallium's existence, many of its properties are far from conventional. Observing the structural motifs of the elemental metals, as illustrated in Fig. 1.1, gallium is the only elemental metal exhibiting an orthorhombic structure in its normal pressure, normal temperature bulk phase. This $\alpha$-gallium orthorhombic unit cell consists of a series of strongly-buckled planes of $\mathrm{Ga}_{2}$ dumbbell with a bond distance of $2.45 \AA$, which is $10 \%$ shorter than the second nearest neighbor at $2.71 \AA$ and leads to the label "molecular metal" [4]. Fig. 1.2 illustrates gallium's unique unit cell with dimers arranged in buckled planes [5], as well as its molecular, facecentered cubic (FCC) nature [6].

The unique properties of gallium also extend to low-temperature and high-pressure modifications, as the element possesses one of the most diverse phase diagrams of any element in the periodic table. Gallium is extremely polymorphic, with solid-state phases taking on a variety of structures as shown in Fig. 1.3. Similar to $\alpha$-gallium, the low temperature $\beta$-, $\gamma$ - and $\delta$-phases are pseudomolecular (as coined by [4]) with the persis- 


\section{Metals by lattice structure}

\begin{tabular}{|c|c|c|c|c|c|c|c|c|c|c|c|c|c|c|c|c|c|c|}
\hline $\mathrm{H}$ & & \multirow{3}{*}{\multicolumn{5}{|c|}{$\begin{array}{r}\text { Hexagonal (HCP } \\
\text { Cubic (BCC } \\
\text { Cubic (FCC } \\
\text { Hybrid HCP/FCC }\end{array}$}} & & & \multirow{3}{*}{\multicolumn{4}{|c|}{$\begin{array}{l}\text { Rhombohedral } \\
\text { Simple cubic } \\
\text { |Tetragonal } \\
\text { Orthorhombic }\end{array}$}} & & & & & & \multirow{2}{*}{\begin{tabular}{|l|}
$\mathrm{He}$ \\
$\mathrm{Ne}$
\end{tabular}} \\
\hline $\mathrm{Li}$ & $\mathrm{Be}$ & & & & & & & & & & & & B & C & $N$ & 0 & $F$ & \\
\hline $\mathrm{Na}$ & $\mathrm{Mg}$ & & & & & & & & & & & & $\mathrm{Al}$ & $\mathrm{Si}$ & $P$ & $S$ & $\mathrm{Cl}$ & $\mathrm{Ar}$ \\
\hline $\mathrm{K}$ & $\mathrm{Ca}$ & $\mathrm{Sc}$ & $\mathrm{Ti}$ & v & $\mathrm{Cr}$ & & & $\mathrm{Fe}$ & Co & $\mathrm{Ni}$ & $\mathrm{Cu}$ & $Z_{n}$ & $\mathrm{Ga}$ & $\mathrm{Ge}$ & As & $\mathrm{Se}$ & $\mathrm{Br}$ & $\mathrm{Kr}$ \\
\hline $\mathrm{Rb}$ & $\mathrm{Sr}$ & $Y$ & $\mathrm{Zr}$ & $\mathrm{Nt}$ & $M c$ & & & Ru & $\mathrm{Rh}$ & Pd & $\mathrm{Ag}$ & $\mathrm{Cd}$ & In & Sn & $\mathrm{Sb}$ & $\mathrm{Te}$ & I & $X e$ \\
\hline Cs & $\mathrm{Ba}$ & $\mathrm{La}$ & $\mathrm{Hf}$ & $\mathrm{Ta}$ & W & & & Os & Ir & $\mathrm{Pt}$ & $\mathrm{Au}$ & $\mathrm{Hg}$ & $\mathrm{TI}$ & $\mathrm{Pb}$ & $\mathrm{Bi}$ & Po & At & $\mathrm{Rn}$ \\
\hline $\mathrm{Fr}$ & $\mathrm{Ra}$ & $A c$ & Rf & $\mathrm{Dt}$ & $\mathrm{Sg}$ & & & $\mathrm{Hs}$ & $\mathrm{Mt}$ & Ds & $\mathrm{Rg}$ & $\mathrm{Cn}$ & Uut & Uuq & Uup & Uuh & Uus & Uno \\
\hline
\end{tabular}

\begin{tabular}{|l|l|l|l|l|l|l|l|l|l|l|l|l|l|}
\hline $\mathrm{Ce}$ & $\mathrm{Pr}$ & $\mathrm{Nd}$ & $\mathrm{Pm}$ & $\mathrm{Sm}$ & $\mathrm{Eu}$ & $\mathrm{Gd}$ & $\mathrm{Tb}$ & $\mathrm{Dy}$ & $\mathrm{Ho}$ & $\mathrm{Er}$ & $\mathrm{Tm}$ & $\mathrm{Yb}$ & $\mathrm{Lu}$ \\
\hline $\mathrm{Th}$ & $\mathrm{Pa}$ & $\mathrm{U}$ & $\mathrm{Np}$ & $\mathrm{Pu}$ & $\mathrm{Am}$ & $\mathrm{Cm}$ & $\mathrm{Bk}$ & $\mathrm{Cf}$ & $\mathrm{Es}$ & $\mathrm{Fm}$ & $\mathrm{Md}$ & $\mathrm{No}$ & $\mathrm{Lr}$ \\
\hline
\end{tabular}

Figure 1.1. Periodic table showing structural trends for the elemental metals.

tence of the $\mathrm{Ga}_{2}$ dimers, indicating a high degree of covalent bonding. In fact, the $\gamma$-phase crystal structure of gallium resembles that of covalently bonded boron. On the other hand, the high-pressure modifications, Ga-II and Ga-III, have high coordination numbers and packing schemes more analogous to metallic elements. In keeping with this trend, a more recent discovery has demonstrated that bulk gallium modifies to a stable FCC structure between 120 and 150 GPa (Ga-IV) [7].

Belonging to Group IIIA in the periodic table, gallium has a $4 s^{2} 3 d^{10} 4 p^{1}$ open shell electronic structure. Bordering both the metallic and covalent elements, bonding in $\alpha$-gallium is also unusual. The dimers present in bulk gallium are covalently bonded. In fact, its dimeric FCC crystal structure, as shown in Fig. 1.2 (b), is identical to that of covalently bonded halogens $\mathrm{Cl}_{2}, \mathrm{Br}_{2}$ and $I_{2}$. This covalency is also hinted at by several experimental studies measuring a pseudogap in the density of states (DOS) that begins just above the Fermi level $[8,9,10]$, with the low number of conducting states defining the gap. This pseudogap has been characterized by 


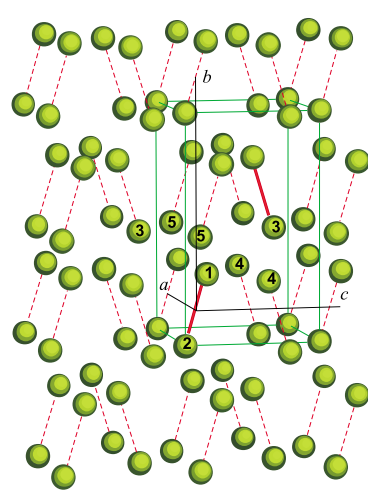

(a)

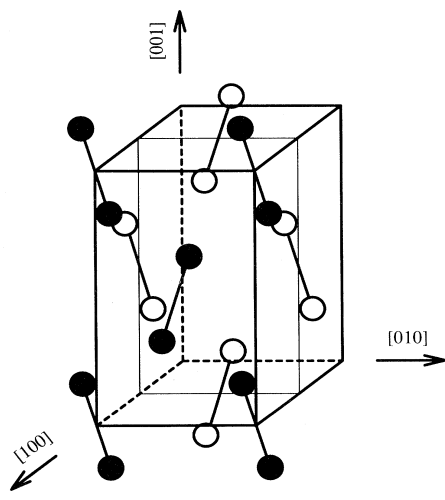

(b)

Figure 1.2. Gallium's unique unit cell, illustrating: (a) the arrangement within the buckled planes of dimers (figure reproduced from [5]) and (b) its molecular face-centered cubic nature, with the dark atoms lying in the ' $a$ ' plane, the white atoms in the a/2 plane, while the ' $a$ '-plane atoms are repeated in the 0-plane (not shown) (figure reproduced from [6]).

a series of theoretical investigations $[11,12,13,6]$, each pointing to the covalent dimers as the culprit. A first-principles investigation completed by Voloshina et al., however, demonstrated that flattening the buckled planes of dimers eliminated the pseudogap, indicating the gap was more strongly influenced by the arrangement of the atoms in the buckled planes [5]. This study clearly demonstrated that the relationship of the electronic structure and lattice structure in gallium is still not fully understood. In contrast to these covalent characteristics, its metallic nature is evident in several macroscopic properties of gallium, such as its luster. Photoemission spectral measurements have confirmed that gallium is a metal, although it possesses the previously mentioned low density of states at the Fermi level $[8,9,10]$. The results of an ab initio study of $\alpha$-gallium indicated that the conducting states present in the gap are formed by the overlap of the wavefunctions along the buckled planes, making these planes metallic in nature [6]. 

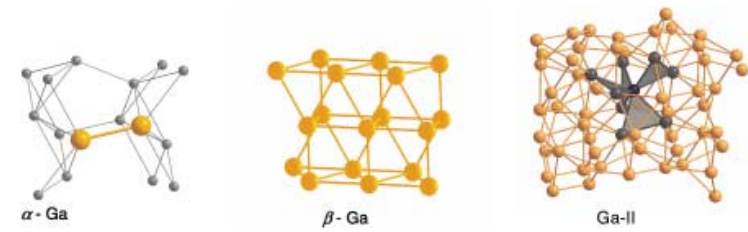

Ga-II
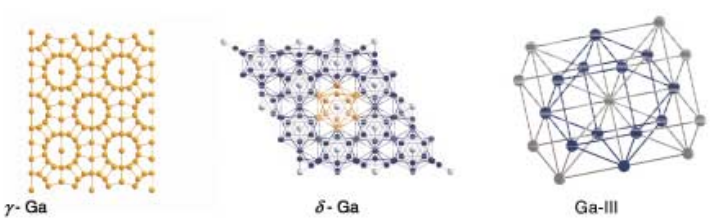

Figure 1.3. Solid-state phases of bulk gallium, reproduced from [4].

Gallium's dual covalent-metallic nature has led to the question of whether bulk gallium's fundamental building blocks consist of the covalent dimers or metallic planes. Experimental studies on the surface properties of $\alpha$ gallium reveal evidence of both, as one low energy electron diffraction study finds a combination of 'short' covalent and 'medium' metallic bonded dimers [14], while x-ray diffraction determined the surface to be comprised of metallic, planar bi-layers [15]. In research on the liquid structure of gallium, one group found evidence of covalency in very short-lived $\mathrm{Ga}_{2}$ dimers upon melting in their first-principles research [16]. These results have been subsequently disputed by two theoretical groups [17, 18], who find evidence of only a small number of unstable dimers in their firstprinciples studies. This result is supported by most experimental [8] and theoretical $[12,19]$ studies on liquid gallium that demonstrate a destruction of short range order and free electron-like behavior.

In argument for dimeric building blocks, a simple study of the bulk cohesive energy as a function of the melting temperature [20] revealed that treating gallium as a metal composed of $\mathrm{Ga}_{2}$ dimers more closely matched the linear trend, as illustrated in Figure 1.4. Treating bulk gallium as metallic-like, composed of Ga-monomers, shows a significantly greater deviation from this trend than the covalent $\mathrm{Ga}_{2}$ model. Overall, it is apparent that gallium takes on properties of both covalent and metal- 
lic systems. This coexisting dual nature is evident in several macroscopic characteristics as well, such as the highly anisotropic thermal and electrical conductivity, with respective ratios of 9:1 and 7:1 from in-plane to along-dimer conductance $[21,22]$.

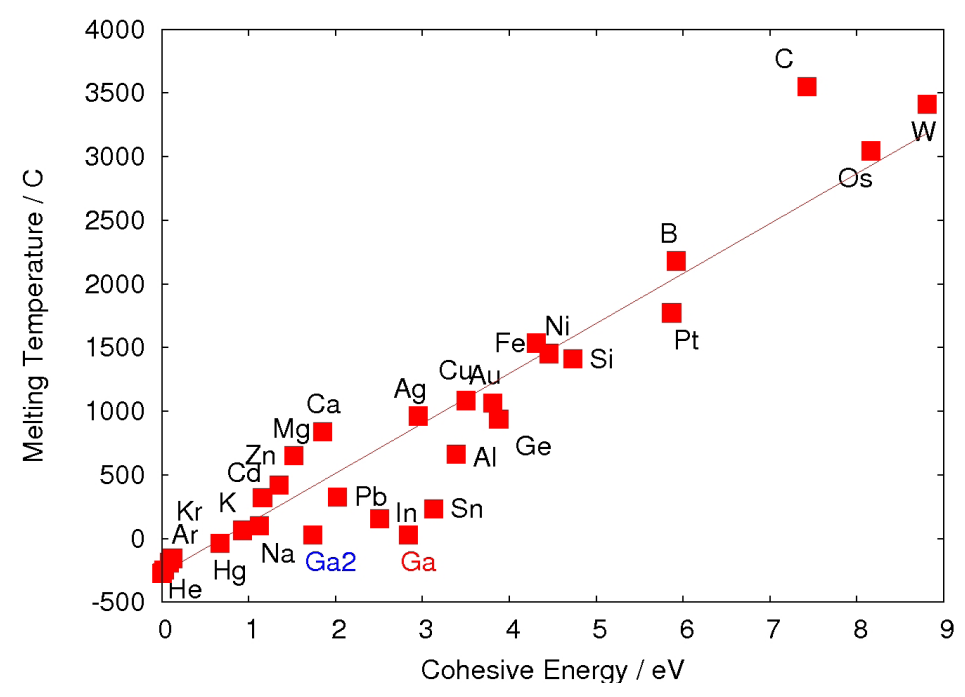

Figure 1.4. Cohesive energy vs. bulk melting temperature for periodic elements, reproduced from [20].

Gallium's thermodynamic properties are also distinct among its metallic neighbors in the periodic table, with an open question as to whether its dual nature factors into these anomalies. With eight atoms per unit cell, gallium has a relatively low atomic density that increases upon melting (like ice). Most bulk systems melt from the surface inward, as surface atoms are less strongly bound than interior (bulk) atoms; however, scanning tunneling microscopic measurements on gallium have found evidence that at least one surface facet of gallium reverses this trend, with its bulk atoms transitioning to a liquid state before the (001) surface atoms $[23,24]$.

Gallium has a high cohesive energy, similar to that of silver, yet the melting temperature is abnormally low for an elemental metal. At $303 \mathrm{~K}$, 
$\alpha$-gallium will melt in a person's hand, as compared to more typical metals such as silver (with similar cohesive energy) or gold with melting temperatures at 1234 and $1337 \mathrm{~K}$, respectively. The origin of the low melting temperature of Ga remains unanswered, with some question as to the role of covalent bonding. Finally, while bulk gallium melts at an intriguingly low $303 \mathrm{~K}$, very small clusters of gallium (around tens of atoms) counter this behavior with melting temperatures between double and triple this temperature. This interesting phenomenon is discussed in greater detail in the following section.

\subsubsection{Small Clusters}

A renewed interest in this fascinating metal came about in 2003 when Breaux et al. published the results of a small cluster melting experiment on gallium. These measurements demonstrated that, when diminished to the size of 10 's of atoms, gallium clusters melt at temperatures up to 2.5 times the bulk melting temperature [25]. This phenomenon is completely contrary to classical behavior as clusters are generally expected to be thermodynamically less stable than their bulk counterparts. Classically, a cluster's melting temperature should decrease with an inverse dependence on cluster radius, clearly demonstrated in Figure 1.5 illustrating the results of a well-known experimental study on gold clusters [26]. This "normal" melting trend can be attributed to the surface atoms being less strongly bound than bulk atoms. As the size of the particles decrease, the surfaceto-bulk ratio increases, lowering the melting temperature accordingly.

While greater-than-bulk melting temperatures have also been observed in isolated sizes of aluminum and tin clusters [27, 28, 29], gallium clusters exhibit the behavior more systematically over a far wider range of cluster sizes. The experiments, outlined in more detail in Section 1.1.3, measured the specific heat curves for gallium cluster cations sized 17, 20, 30-50, and 55 atoms [30, 25, 31, 32], with Fig. 1.6 illustrating the results for the largest 


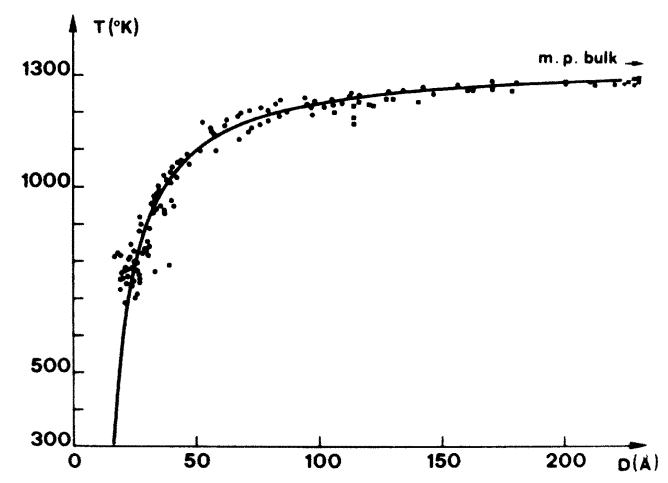

Figure 1.5. Gold clusters following the expected melting temperature trend, $\sim 1 / \mathrm{r}$ (figure reproduced from [26]).

clusters. The melting phase transition is defined by the peak in the specific heat curve, with the height and width of the curve depend on the way in which the cluster responds to increasing temperatures. The melting transition generally occurs with either a sharp increase in the latent heat over a short temperature range or more slowly giving off latent heat over a broader temperature range. While the specific heat melting peak in a bulk system is expected to be nearly a $\delta$ function, finite size effects broaden the peak in clusters [33]. For cluster sizes without a sharp peak, such as the 30or 50-atom cluster, additional measurements of the cluster's cross sectional area, as illustrated in Fig. 1.7, show a deviation from the low-temperature trends very near where the specific heat is at a maximum. This inflection in cross section is associated with the cluster transitioning to a liquid, as a liquid has a larger coefficient of expansion [31]. It is expected that these clusters do, in fact, melt but do so without a significant release of latent heat [31].

Each of the experimentally measured clusters exhibits a specific heat peak and/or cross sectional change between 500 and $800 \mathrm{~K}$. Additionally, within the size regime of 30-50 atoms, the addition or removal of a single atom can alter the cluster melting temperature by more than $100 \mathrm{~K}$, as demonstrated in Figure 1.8 [34]. These one-atom changes often trans- 


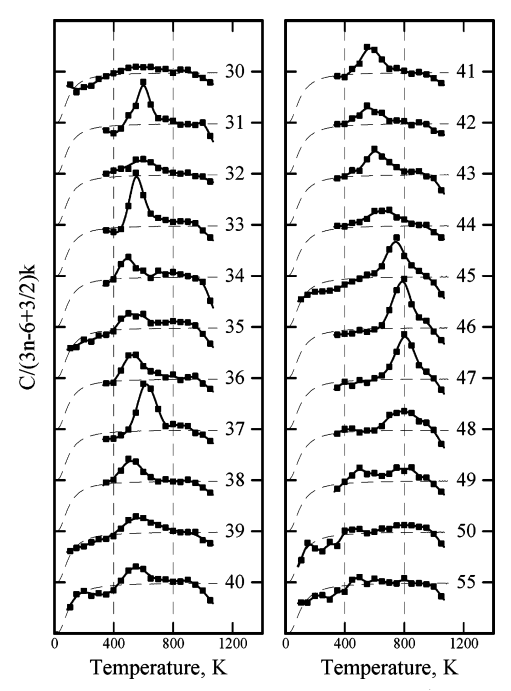

Figure 1.6. Experimental specific heat curves for $\mathrm{Ga}_{n}(\mathrm{n}=30-50,55)$ (reproduced from [34]).

form a cluster from a "magic melter" (as coined in Ref. [30]) exhibiting a finely-peaked specific heat curve with a clear melting transition, into a "non-melter" with a widely-broadened, poorly defined peak. For the size range of 30-34 atoms, there appears to be an odd-even oscillation to the specific heat natures (Fig. 1.6), while this oscillation appears in the melting temperatures between 36-43 atoms (Fig. 1.8).

While experimental results have clearly demonstrated the greater-thanbulk melting and size-sensitivity for these small, interesting clusters, computational and theoretical research has yet to uncover a firm basis for the effect. The role of gallium's dual covalent-metallic nature in this anomalous thermodynamic behavior remains an open question. The source of the melting temperature anomalies observed for this element has been attributed to a difference in the nature of bonding between the bulk and clusters [35], although the nature of this difference has not been clearly established. An overview of the theoretical and computational efforts directed toward understanding this phenomenon will be outlined in greater 


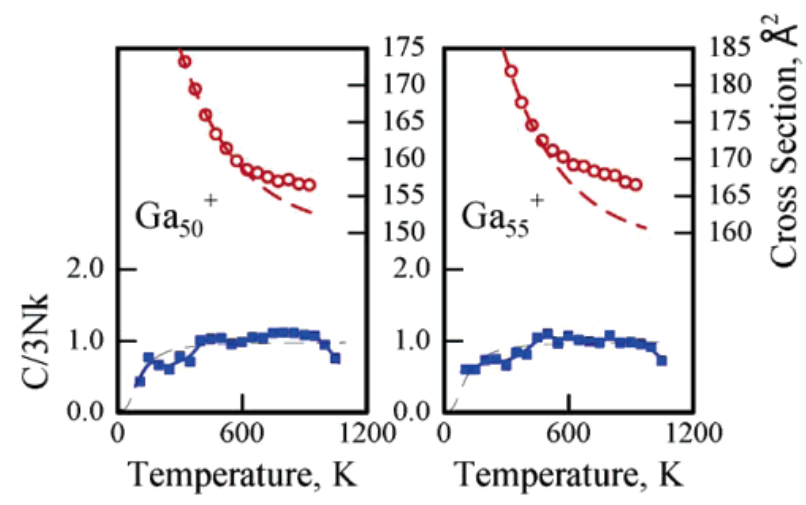

Figure 1.7. Experimental measurements for $\mathrm{Ga}_{50}$ and $\mathrm{Ga}_{55}$, illustrating the specific heat measurements as well as cross sectional changes, with the red dashed lines representing fits to the cross sectional curves using a simple exponential model. Although the specific heat curves do not exhibit a well-defined peak, the cross sectional areas indicate that a melting transition has occurred (figure reproduced from [31]).

depth in Section 1.1.5, while the following section outlines the experimental methods leading to this intriguing discovery.

\subsubsection{Experimental measurements}

While the experimental procedures are outlined in great depth in Refs. [36, 37] and summarized as they apply to small clusters in Refs. [25, 34, 31, 32], a brief overview will be provided here. The specific heat measurement methods employed by the Jarrold group for the small gallium clusters can generally be broken down into 5 basic phases: cluster generation, heating, size selection, helium bombardment and a second size selection. In order to generate the clusters, the group uses a pulsed laser striking bulk gallium contained in a vacuum chamber. The clusters are then moved through a variable temperature extension. In reaching thermal equilibrium with the walls of the extension, the temperature of each cluster is set. They then pass through a quadrupole mass spectrometer in order to select the size of 


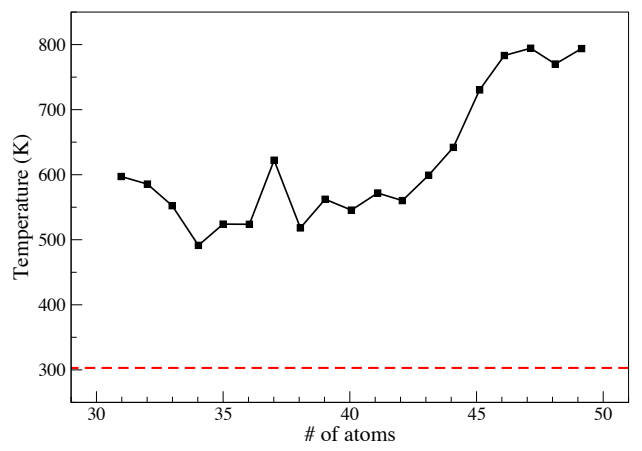

Figure 1.8. A summary of the melting temperatures of gallium clusters sized 30-50 atoms (black solid) [34], relative to the melting temperature of bulk gallium (red dashed).

the clusters to be measured. The clusters are accelerated through an electric field to set their kinetic energy, into a collision cell where they are thermalized by high pressure helium gas. When the cluster enters the collision cell, it undergoes numerous collisions with the helium gas, whereby small portions of its kinetic energy are translated into internal energy. Given a sufficient translational (kinetic) energy, some portion of clusters will gain enough internal energy to dissociate. After a number of collisions, both the ions as well as their dissociated fragments lose all translational energy (are thermalized), at which point they are drawn across the collision cell by a weak electric field. Finally, they pass through a second quadrupole mass spectrometer where they are size-selected a second time for measurement.

Overall, the experiments measure internal energy as a function of temperature. Given the statistical nature of the measurements and the acknowledgement that clusters inherently have a broadened specific heat peak due to finite size effects, the Jarrold group uses a measurement of the kinetic energy at which $50 \%$ of the clusters dissociate in order to quantify the specific heats. Fig. 1.9 gives a cartoon overview of the procedure; however, several steps will be outlined in greater detail here.

After generating the clusters with the pulsed laser and size selecting 
with the first mass spectrometer, the temperature of each cluster is set within the variable temperature extension. The cluster is then accelerated to a particular kinetic energy into a high pressure helium collision cell. At each kinetic energy, the percentage of clusters that have dissociated is measured, as illustrated in Fig. 1.9 (b). These \%-dissociation values create a linear trend, illustrated in (c), from which the kinetic energy yielding TE50\% dissociation (translational energy for $50 \%$ dissociation) can be obtained. The temperature within the variable temperature extension is then changed and the series of kinetic energy measurements are repeated yielding a series of $50 \%$ dissociation values, each corresponding to a temperature, as shown in (d). This curve is analogous to a caloric curve, and its derivative is directly proportional to the specific heat measurements, as illustrated in (e). The proportionality constant, outlined in greater depth in Ref. [37].

The Jarrold group completed extensive measurements for gallium, repeating the procedure several times for each cluster cation sized 17, 20, 30-50 and 55 atoms. The greater-than-bulk melting behavior was observed for all cluster sizes measured, firmly establishing the trend across a large range of sizes. While this thermodynamic phenomenon has been well documented experimentally, a theoretical understanding of the underlying physics and chemistry governing this behavior has yet to be established. Generally, thermodynamic calculations are completed using either Monte Carlo or molecular dynamics simulations. As this research utilizes molecular dynamics (MD), the following section introduces classical MD modeling of greater-than-bulk melting.

\subsubsection{Classical simulations}

Much research has been completed using classical molecular dynamics to model cluster melting, yet very few simulations have been able to classically capture "greater-than-bulk" melting temperatures. Nearly all Newtonian- 
a)

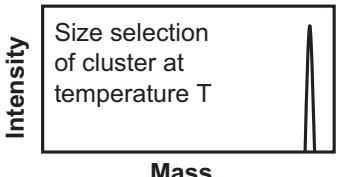

b)

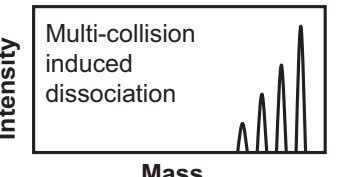

c)

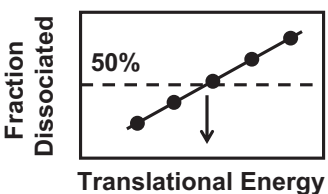

d)

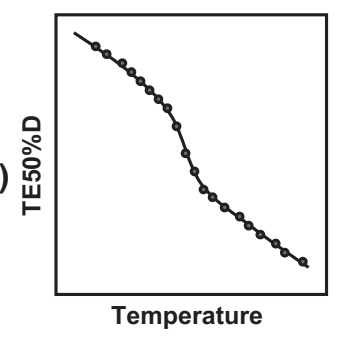

e)

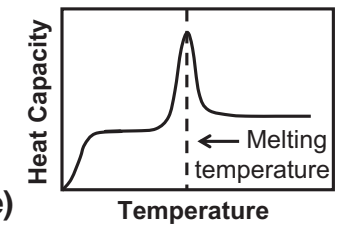

Figure 1.9. A cartoon reproduced from Ref. [37] in order to illustrate the phases of the specific heat measurements via ion drift tube measurement techniques: a) the size selection by the first mass spectrometer; b) the dissociation process by which a percentage of the original size-selected clusters are fragmented into smaller cluster sizes; c) a representation of the $\%$-dissociation values for a range of kinetic energies at a given temperature; d) a representative plot of the TE50\% dissociation values for a range of temperatures; and e) the derivative of the TE50\% dissociation values, which is proportional to the specific heat.

based MD simulations yield the classic 1 / $\mathrm{r}$ cluster melting point depression illustrated in Figure 1.5. This is understandable given that most classical MD potentials are empirically developed, fitted to experimental data describing macroscopic properties. For this reason, classical MD simulations are more commonly used for larger clusters, as classically-derived potentials are generally considered inadequate for the smaller cluster regime.

Over the past couple of decades, the embedded atom method (EAM) potentials $[38,39,40]$ have gained increasing popularity for cluster melting simulations in the classical realm, particularly for metallic systems. 
Generally, EAM potentials can be described by the equation

$$
E_{\text {tot }}=\sum_{i} F_{i}\left\{\rho_{i}\left(R_{i}\right)\right\}+\frac{1}{2} \sum_{i, j} \varphi\left(R_{i j}\right)
$$

where $\rho_{i}$ is the electron density of the host without atom $i, F_{i}$ is a functional of that electron density and $\varphi\left(R_{i j}\right)$ is the short-range electrostatic pair potential. With the additional functional $F_{i}$, EAM potentials treat each electron as an impurity within the sea of electron density, thus creating something between a simple pair potential and a more complex many-body potential accounting for electron-electron interactions.

Using one such EAM potential, superheating was identified in aluminum clusters sized 586 to 37,786 atoms [41, 42]. Their microcanonical simulations employed an EAM glue potential, developed specifically for aluminum by numerically fitting large amounts of first-principles data [43]. Through these simulations, Schebarchov et al. were able to delineate a finite size range for superheating in aluminum clusters of 5-9 $\mathrm{nm}$. While the glue potential served well in simulating superheating for larger aluminum clusters, both the glue potential and second EAM potential (the Gupta potential) proved insufficient when Noya et al. attempted to model the melting behavior of smaller $\mathrm{Al}_{n}$ clusters $(\mathrm{n}=49-62)$ [44].

Given the small system size and inherent complexities, previous research on greater-than-bulk melting in small gallium clusters turned to a quantum mechanical-based model. First-principles and ab initio modeling, introduced in the following section, represent two such models.

\subsubsection{First-principles and $a b$ initio modelling}

Given the small size regime and element-specific behaviors, most studies have tried to address the issues of size-sensitivity and greater-thanbulk melting temperatures in small gallium clusters using density functional theory (DFT or first-principles) or Hartree Fock (HF)/post-HF ( $a b$ initio) calculations. These investigations have probed both finite tempera- 
ture atomic and electronic structure, the dynamics of the phase transition through first-principles molecular dynamics (FPMD) simulations as well as extensive global minimum (GM) searches.

In summary, most small gallium FPMD studies have concluded that the greater-than-bulk melting temperatures arise from the difference in the nature of bonding between bulk and small clusters [35, 45, 32]. The bulk of the FPMD research has pointed to a covalency within the clusters, claiming that networks of covalently bonded atoms are responsible for the increased cluster melting temperatures. However, evidence supporting this covalency has been subsequently questioned [46].

The size-sensitivity has been attributed to a relative measure of (local) order versus disorder in the ground state cluster structure [47, 32]. As an example, Joshi et al. completed a first-principles based molecular dynamics study simulating the specific heat curves for $\mathrm{Ga}_{30}$ and $\mathrm{Ga}_{31}$ [47]. They attempt to explain the differences between the natures of the melting transitions using a relative measure of order between the ground state structures of the clusters. Their argument is based on the claim that $\mathrm{Ga}_{31}$ is more "highly ordered" than $\mathrm{Ga}_{30}$. Being more ordered, $\mathrm{Ga}_{31}$ has a lower number of accessible states than $\mathrm{Ga}_{30}$ at low temperatures and, therefore, demonstrates a more finely peaked melting transition. The modelled specific heat curves as well as their GM structures are illustrated in Fig. 1.10.

Generally speaking, this emphasis on relative order and disorder in ground state structures is problematic for gallium due to the extreme polymorphism known for bulk gallium [4]. A diverse range of structures has also been noted in small gallium clusters [48, 49, 50, 51], implying complicated potential energy surfaces for these small systems. While ground state structural analysis has been employed to explain the anomalous thermodynamic behavior observed in small sodium $[52,53,54,55,56]$ and aluminum structures $[57,58,35]$, it presents several unique challenges due to the low numbers of atoms in these small clusters. Structural measures of order more commonly seen for larger clusters, such as common neighbor 


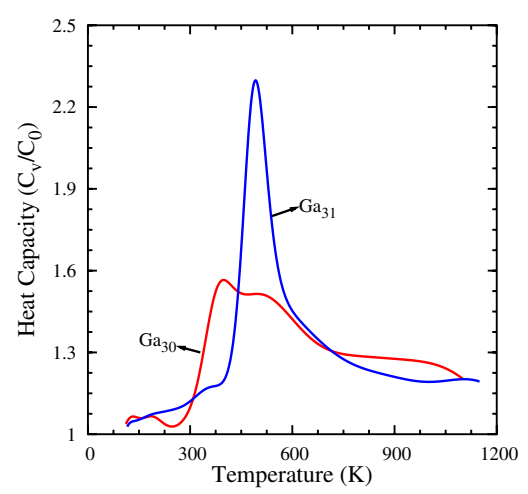

(a)

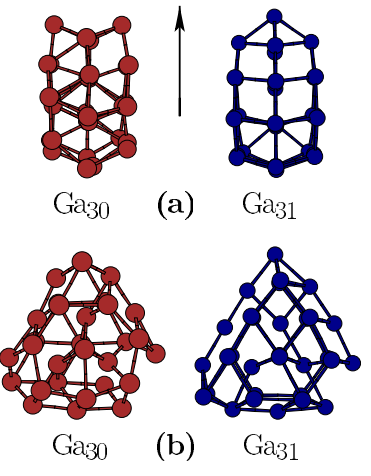

(b)

Figure 1.10. (a) First-principles molecular dynamics simulation results for $\mathrm{Ga}_{30}$ and $\mathrm{Ga}_{31}$; and (b) global minimum (GM) structures for the two clusters. Both figures are reproduced from [47].

analysis $[59,60,61]$ or the Steinhardt order parameter $[62,63]$ typically break down with so few atoms where a bulk-like environment can not be defined. Without the aid of such quantitative measures, explaining the size-sensitivity in small gallium cluster thermodynamics by measures of structural order versus disorder becomes a largely qualitative assessment.

The size-sensitivity observed for small gallium clusters has also been attributed to the number and energy distribution of the low-energy isomers of a cluster, as identified during extensive searches for GM structures [32]. The isomer contribution to the melting signature is more clearly illustrated in Fig. 1.11, which shows the experimental results for the 17- and 20atom cations as well as low-energy isomer distributions for these two clusters. The specific heat curve for $\mathrm{Ga}_{17}^{+}$has a non-melting character, without a clearly defined peak while $\mathrm{Ga}_{20}^{+}$exhibits a clear melting transition at $\sim 700 \mathrm{~K}$. The isomer energies for the 17-atom cation increase only slightly, with a large number of structures falling within $0.2 \mathrm{eV}$ of the ground state, while the low-energy isomers for the 20-atom cation progress in large energy steps. This leads to the conclusion that as the 17-atom cation melts, it 
transitions easily between each of the isomers, thus melting more gradually without a significant increase in latent heat, while the 20-atom cation transitions more abruptly between isomers with far more latent heat [32].

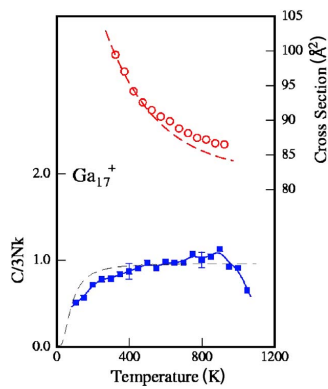

(a)

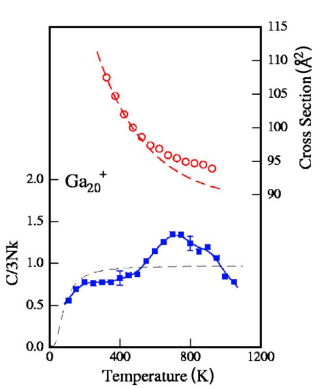

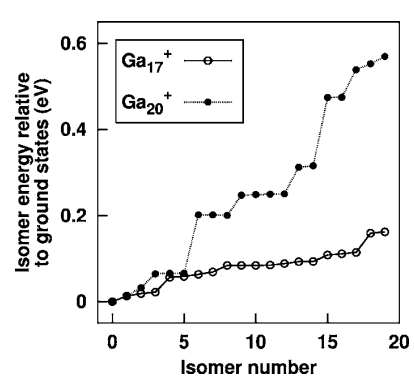

(b)

Figure 1.11. A combined experimental and first-principles study relating (a) the nature of the experimental specific heat curve to (b) the number and distribution of low-energy isomers. Both figures are reproduced from [32].

Many of the FPMD calculations completed to date have been performed for neutral gallium clusters [45, 47, 64], drawing direct comparisons to experimental measurements completed for charged gallium cluster. These comparisons are made on the basis of several theoretical studies employing classical potentials to probe the effect of charge on the overall thermodynamic behavior of small clusters [53]. To summarize an often cited study, Calvo and Spiegelmann completed a thorough theoretical investigation of small $\mathrm{Na}_{n}$ and $\mathrm{Na}_{n}^{+}$clusters, including simulations for 12 cluster sizes ranging from 8 to 147 atoms, demonstrating that the effect of charge was negligible for both the melting temperatures and overall nature of the melting transition for most cluster sizes within the framework of their model [53]. However, a first-principles study completed for small sodium clusters demonstrated that the addition of just one electron could alter the melting temperature by as much as $40 \mathrm{~K}$ as well as change the overall nature of the specific heat curve from a low, broad melting transition to a 
finely-peaked one [65], attributing these differences to the electronic structure as well as the distribution of low-energy isomers.

An experimental study measuring the melting temperatures of small aluminum cations and anions verified that, for many cluster sizes, charge had very little effect on both the melting temperature and overall nature of the specific heat curves [57]. This same study, however, clearly demonstrated that the effect of charge was profound for certain cluster sizes, altering the melting temperature by as much as $500 \mathrm{~K}$. To date, the only FPMD studies addressing the effect of charge on small gallium cluster melting models include the $\mathrm{Ga}_{17}$ [45] and $\mathrm{Ga}_{17}^{+}$[32] simulations. According to these studies, both clusters have a similar ground state geometry and the low-lying isomers span a similar range of $0.8 \mathrm{eV}$, leading to the expectation that the cationic and neutral clusters would exhibit similar melting transitions [45]. These simulations, however, yield notably different specific heat curves, with the charged cluster as a non-melter exhibiting no clear specific heat curve peak while the neutral cluster had a distinct peak around $600 \mathrm{~K}$. No direct comparison drawn between the two simulations to explain these differences.

A series of global minimum (GM) studies on small gallium clusters have also been completed, attempting to investigate the electronic and structural contributions to the melting anomalies [48, 49, 50, 51, 46]. Ab initio and DFT geometry optimizations indicate that the dimeric nature observed in bulk gallium persists in small gallium clusters [48, 49, 50]. Figure 1.12 shows the ground state structures for $\mathrm{Ga}_{4}-\mathrm{Ga}_{8}$ neutral clusters and ions, as well as the non-ground state singlet structure for $\mathrm{Ga}_{4}$. Each of the clusters strongly favors structures composed of dimers [49]. This study theorizes that the dimeric trend for small gallium structures could factor into the odd-even melting temperature oscillation observed for larger gallium clusters.

Each of the previously mentioned studies has implicated various atomic and electronic structural contributions in greater-than-bulk melting and 

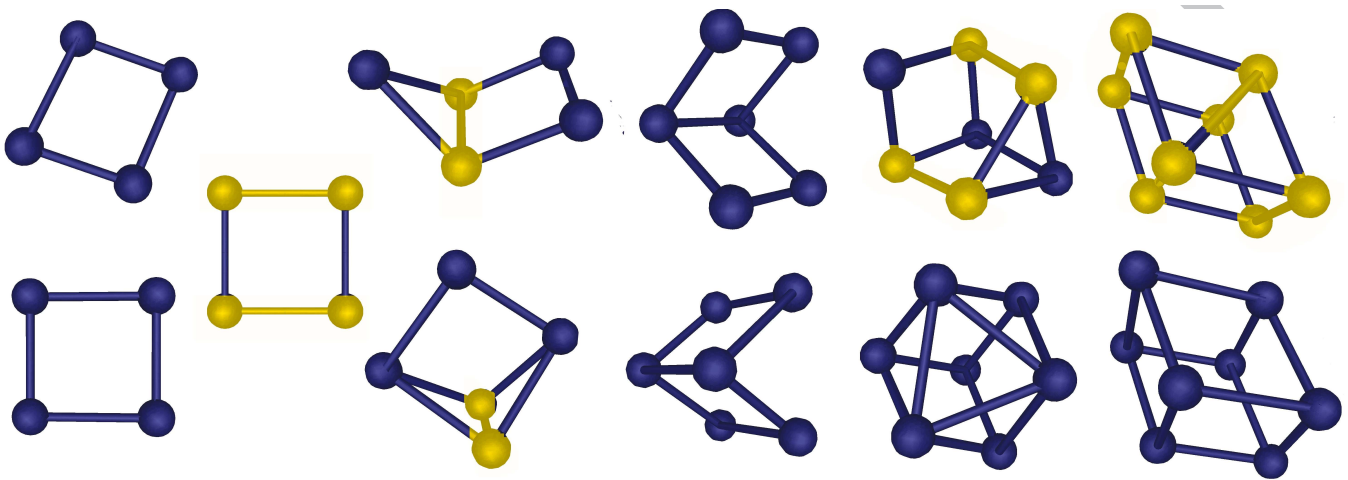

Figure 1.12. Ground state structures for gallium clusters for neutral (top row) and cationic (bottom row) clusters, reproduced from [49]. Pairs of atoms highlighted in yellow are within $5 \%$ of the nearest neighbor distance for bulk gallium.

size-sensitivity. The influence of gallium's dual metallic-covalent nature has been extensively investigated, with no conclusive evidence to support claims that either covalency or metallicity plays a role in either the elevated melting temperature of the clusters or the dramatic size-sensitivity. This research project utilizes extensive FPMD simulations coupled with atomic and electronic structural analysis to investigate these intriguing questions. Although this research was directed primarily at analyzing the structures and properties of the very smallest molecular systems, understanding the contributions to molecular structure at the other end of the size spectrum was important to formulating a complete picture of molecular solids. Calculations of N-body contributions to the cohesive energy of the solid halogens, as introduced in the following section, provided additional insight into the nature of bonding in molecular solids. 


\subsection{Solid Halogens}

Sharing gallium's unique molecular bulk structure are the solid halogens, chlorine, bromine and iodine. As group 17 elements, each of the solid halogens has seven valence electrons, just one electron shy of the noble gases. Under standard atmospheric conditions, these three elements exist in each of the three phases: chlorine as a gas (becoming solid below $88 \mathrm{~K}$ [66] or at high pressures), bromine as a liquid (becoming solid below 265 $\mathrm{K}$ [67] or at high pressures) and iodine as a solid.

In the solid form, each of these halogens are crystallographically isomorphic, with only changes in the length of the unit cell parameters $a$, $b$ and $c$ differentiating between the structures. With Cmca space group symmetry and two molecules per primitive unit cell, atomic nearest neighbor distances between diatomic pairs are nearly $60 \%$ shorter than nearest molecular neighbor distances. This makes the intramolecular pair bonding in the solid halogens quite strong in comparison to the van der Waals forces that govern the intermolecular bonds creating the solid. Further complicating bonding in the solid halogens, the intermolecular atom-atom distances are far shorter than the van der Waals radius, at $0.8 r_{v d W}$ for $\mathrm{I}_{2}$, $0.6 r_{v d W}$ for $\mathrm{Br}_{2}$ and $0.25 r_{v d W}$ for $\mathrm{Cl}_{2}$ [68].

This unique structure has generated extensive experimental interest over the past century. Similar to gallium, the role of covalency in enhancing the stability of the Cmca orthorhombic structure has been questioned. A series of theoretical studies have shown that models reproducing the Cmca structure indicate that both the inter- and intramolecular bonds are covalent in nature $[69,70,71]$. Much of the recent research on the solid halogens has related to structural modifications under pressure. Under high pressure conditions, each of the solid halogens undergoes a molecular dissociation phase transition, changing from a molecular to monatomic solid as the inter- and intramolecular distances become indistinguishable. A number of changes occur with the onset of this transition, to include 
a decrease in the band gap and increase in metallicity [72, 73, 74, 71, 75]. Combining $x$-ray diffraction with conductivity measurements, it was determined that iodine undergoes a transition to a fully metallic state at between 16-21 GPa [72,75]. Although this transition has not been observed for bromine or chlorine, it was found that bromine began to reflect light at $\sim 60 \mathrm{GPa}$, possibly indicating a change to a metallic state at that pressure [75].

Theoretical interest in the crystal structure of these solids has been extensive, with the first calculations attempting to capture their Cmca lattice structure dating back to the early 1960's. These studies have employed a variety of potentials and methods, to include classical models (citing only a few of many) [76, 77, 78, 79, 69, 68, 80], first-principles models [81, 82] as well as ab initio models $[83,84]$. Astoundingly, despite the substantial body of work over the past $60+$ years directed at trying to understand the molecular structure of the solid halogens, a solid theoretical model that accurately predicts all the properties of the solid halogens is still lacking.

In an attempt to probe the nature of bonding for the solid halogens, thereby gaining insight into gallium's unique molecular structure, method of increments calculations were completed for $\mathrm{Cl}_{2}, \mathrm{Br}_{2}$ and $\mathrm{I}_{2}$. This method calculates a series of $\mathrm{N}$-body correlation energies that serve as incremental corrections to an ab initio (Hartree-Fock) cohesive energy.

The following chapter provides an overview of the theory underlying the approximate quantum chemical methods used to investigate both greater-than-bulk melting in gallium clusters, as well as the N-body contributions to the cohesive energy of the solid halogens. Chapters 3 through 7 outline the initial testing completed for gallium clusters, methods utilized for thermodynamic modeling, as well as the results for $\mathrm{Ga}_{20}, \mathrm{Ga}_{n}$ microclusters $(n=7-12)$ and larger $\mathrm{Ga}_{N}$ clusters $(\mathrm{N}=32-35)$. Chapter 8 outlines the methods and results of method of increments calculations for the solid halogens, providing additional details on the nature of bonding in molecular solids. Finally, Chapter 9 provides a summary of the research, 
outlining how this research has furthered our understanding of bonding in molecular solids ranging from the smallest cluster sizes to bulk systems. 


\section{Chapter 2}

\section{Theory}

As introduced in the previous chapter, the methods used to calculate the properties of both gallium clusters and the bulk solid halogens include approximations to the exact mathematical formulation. An introduction to the exact equations must precede approximations and thus, this story begins with the full Hamiltonian, represented in atomic units as

$$
\begin{aligned}
\hat{H}_{e N}=-\sum_{i=1}^{N} \frac{1}{2} \nabla_{i}^{2}-\sum_{A=1}^{K} \frac{1}{2 M_{A}} \nabla_{A}^{2} & +\sum_{A=1}^{K} \sum_{B>A}^{K} \frac{Z_{A} Z_{B}}{\left|\vec{R}_{A}-\vec{R}_{B}\right|}-\sum_{i=1}^{N} \sum_{A=1}^{K} \frac{Z_{A}}{\left|\vec{r}_{i}-\vec{R}_{A}\right|} \\
& +\sum_{i=1}^{N} \sum_{j>i}^{N} \frac{1}{\left|\vec{r}_{i}-\vec{r}_{j}\right|}
\end{aligned}
$$

where the indices $i$ and $j$ represent electron terms, $A$ and $B$ represent ion (nuclear) terms, $N$ is the total number of electrons, $K$ is the total number of ionic cores, $M_{A}$ is ratio of the mass of ion $\mathrm{A}$ to the mass of an electron, $Z$ is the atomic number, $\vec{R}$ give the ionic coordinates and $\vec{r}$ represent the coordinates of the electrons. The first term represents the kinetic energy of the $i$ th electron and involves taking the gradient with respect to its position. The second term is the corollary for the $A$ th nucleus. The final three terms represent the Coulomb interactions between (in order of appearance): two nuclei, $A$ and $B$; nucleus $A$ and electron $i$; and two electrons, $i$ and $j$. To 
avoid confusion, it should be mentioned that the charge of the electrons as well as physical constants that would normally be seen in certain terms, such as $\epsilon_{0}$ (vacuum permittivity) and $\hbar$ (reduced Plank's constant), have been absorbed within the 'atomic units' representation in scaling $\vec{r}$ by the Bohr radius as

$$
\overrightarrow{r^{\prime}}=\frac{e^{2} m}{4 \pi \epsilon_{0} \hbar^{2}} \vec{r}
$$

The Hamiltonian given in Eq. (2.1) yields the non-relativistic Schrödinger equation,

$$
\begin{aligned}
\hat{H}_{e N} \Psi & =E_{T} \Psi \\
{\left[\hat{T}_{N}+\hat{T}_{e}+\hat{V}_{N N}+\hat{V}_{e N}+\hat{V}_{e e}\right] \Psi } & =E_{T} \Psi,
\end{aligned}
$$

with $\hat{T}$ designating kinetic energy operators, $\hat{V}$ representing potential energy operators, subscripts of $N$ for nuclear terms and $e$ for electron terms, while $E_{T}$ represents the total energy and $\Psi$ is the wavefunction.

Eq. (2.3) forms the foundation for all computational quantum chemistry approximations. The first two terms, $\hat{T}_{N}$ and $\hat{T}_{e}$, are inherently onebody terms. While the Born-Oppenheimer approximation (as summarized in the following section) gives a first step towards solving the full, non-relativistic Schrödinger equation in rendering the nuclear-nuclear and nuclear-electron Coulomb terms, $\hat{V}_{N N}$ and $\hat{V}_{e N}$, also one-body terms, the final electron-electron term remains a many-body problem that is theoretically and computationally arduous. Most computational quantum chemical algorithms have been developed in order to solve or, more often, approximate the total energy arising from electron-electron many-body interactions.

This chapter presents an overview of the many-body electron problem within computational quantum chemistry, as well as a general outline of several methods addressing the problem. Secs. 2.1 and 2.2 present 
the relevant mathematical background and framework, while Secs. 2.3 through 2.5 give an overview of three methods, Hartree-Fock, Density Functional Theory and the Method of Increments, employed here to address the electron-electron many-body problem.

\subsection{Born-Oppenheimer Approximation}

In observing any molecular system, two distinct classes of particles can be defined on the basis of mass: massive ionic cores and comparably light electrons. The cumbersome ionic cores move quite slowly compared to the highly mobile electrons, which react quickly in response to changes within the system. These distinct velocity profiles led Max Born and Robert Oppenheimer, in 1927, to devise a method that divides the solution to the full, non-relativistic Schrödinger equation into two simplified steps. In the first step, the ground-state electronic configuration is computed for a given ionic configuration. These ground-state electron positions define a potential energy surface by which the positions of the nuclear cores are updated in the subsequent step. Known as the Born-Oppenheimer (BO) approximation [85], this separation greatly simplifies the solution to the full Hamiltonian.

Following the development outlined in Refs. [86, 87], ${ }^{1}$ a general mathematical description begins with the full, non-relativistic Schrödinger equation for the electron-ion system as shown in Eq. (2.3). Explicitly designating the electron positions, $\vec{r}$, and the nuclear positions, $\vec{R}$, this equation becomes

$$
\left[\hat{T}_{N}+\hat{T}_{e}+\hat{V}_{N N}(\vec{R})+\hat{V}_{e N}(\vec{r}, \vec{R})+\hat{V}_{e e}(\vec{r})\right] \Psi(\vec{r}, \vec{R})=E \Psi(\vec{r}, \vec{R}) .
$$

As the $\hat{V}_{e N}$ coupling term is relatively large, it cannot be ignored and renders Eq. (2.4) inseparable. The BO approximation then makes use of the

\footnotetext{
${ }^{1}$ Ref. [86], pgs. 43-45.
} 
adiabatic theorem $[88,89]$, which states that if the external environment of a quantum system is varying slowly enough, the solution to the wavefunction will adopt its unperturbed form. As it applies to the BO approximation, this theorem is fulfilled as the ions are assumed to be moving significantly slower than the electrons due to their larger mass. The electronic wavefunction can then be solved assuming fixed ionic cores. In terms of Eq.(2.4), the adiabatic theorem means that $\vec{R}$ can now be treated parametrically, where the ground-state solution to the electronic wavefunction remains constant for a given $\vec{R}$ but will be distinct for each unique ionic configuration.

The combined electron-ion wavefunction can then be separated into two distinct components:

$$
\Psi(\vec{r}, \vec{R})=\Phi(\vec{r} ; \vec{R}) \chi(\vec{R}),
$$

with $\Phi(\vec{r} ; \vec{R})$ representing the electronic wavefunction, parametrically dependent on $\vec{R}$, and $\chi(\vec{R})$ as the wavefunction for the ionic cores. The decoupled electronic Schrödinger equation can now be written as

$$
\left[T_{e}+V_{e e}(\vec{r})+V_{e N}(\vec{r}, \vec{R})\right] \Phi(\vec{r} ; \vec{R})=\varepsilon_{e}(\vec{R}) \Phi(\vec{r} ; \vec{R})
$$

where $\varepsilon_{e}(\vec{R})$ gives the electronic energy eigenvalues depending parametrically on $\vec{R}$. These electronic energy eigenvalues directly define an effective electronic field,

$$
\varepsilon_{e}^{\mathrm{eff}}(\vec{R})=\varepsilon_{e}(\vec{R})
$$

by which the ionic positions can be updated. The decoupled nuclear Schrödinger equation can now be written as

$$
\left[T_{N}+V_{N N}(\vec{R})+\varepsilon_{e}^{\mathrm{eff}}(\vec{R})\right] \chi(\vec{R})=\mathscr{E} \chi(\vec{R}),
$$


where $\mathscr{E}$ represents the BO approximation to the total energy of the system.

The BO approximation greatly simplifies the solution to the full Schrödinger equation in transforming the electronic wavefunction into a problem of $N$ electrons operating in a field of $K$ fixed, positive point charges. However, it leaves the issue of the electron-electron many-body term unresolved. Although there are many algorithms addressing this troublesome term, one of the more basic, original methods still extensively used is the HartreeFock, or mean-field, approximation, to be addressed in Section 2.3. The following section offers an overview of the relevant mathematical background necessary for a basic understanding of each of the subsequent methods.

\subsection{Mathematical Background}

In order to properly understand any quantum chemical theory addressing the problem of accurately calculating the electron-electron interaction energy, a brief overview of mathematics and terminology is first required. Closely following the development outlined in Ref. [86], an outline of the mathematical background formulating the basis of many computational quantum chemistry methods begins with electron spin. A proper electronic wavefunction must address the issue of spin, which the previous equations neglect entirely. For example, the wavefunction for an $N$-electron system, as described by Eq. (2.6), would take on the form

$$
\Phi(\vec{r} ; \vec{R})=\Phi\left(\vec{r}_{1}, \vec{r}_{2}, \ldots, \vec{r}_{j}, \ldots, \vec{r}_{k}, \ldots \vec{r}_{N} ; \vec{R}\right),
$$

which varies solely according to the positions of the individual electrons, with $\vec{r}_{1}$ representing the position of electron 1 and so on. Accounting for spin, the derivation introduces a new variable, $\vec{x}$, which represents both

${ }^{2}$ Pgs. 45-53. 
the spin and position variables for each electron. Taking the parametric dependence on the nuclear positions, $\vec{R}$, as a given, Eq. (2.9) can be transformed into

$$
\Phi(\vec{x})=\Phi\left(\vec{x}_{1}, \vec{x}_{2}, \ldots, \vec{x}_{j}, \ldots, \vec{x}_{k}, \ldots \vec{x}_{N}\right) .
$$

With the introduction of coordinates accounting for the electron spin state, antisymmetry, spin orbitals, the Hartree product and Slater determinants can now be defined, as outlined in the following sections.

\subsubsection{Antisymmetry}

A proper description of any electronic wavefunction must include the property of antisymmetry, which means that the interchange of any two identical fermions (here, electrons with the same spin) must change the sign of the wavefunction. For example, assuming the $j$ th and $k$ th electrons from Eq. (2.10) are indistinguishable, exchanging them must yield

$$
\Phi\left(\vec{x}_{1}, \vec{x}_{2}, \ldots, \vec{x}_{j}, \ldots, \vec{x}_{k}, \ldots \vec{x}_{N}\right)=-\Phi\left(\vec{x}_{1}, \vec{x}_{2}, \ldots, \vec{x}_{k}, \ldots, \vec{x}_{j}, \ldots \vec{x}_{N}\right) .
$$

It is important to note that one can only exchange electrons of parallel spin, as two electrons with opposite spin are inherently non-identical.

\subsubsection{Spin Orbitals}

A spatial orbital is a well-known construct within the fields of quantum physics and chemistry. The spatial orbital $\phi_{i}(\vec{r})$ defines the spatial distribution for the $i$ th electron, such that $\left|\phi_{i}(\vec{r})\right|^{2} d \vec{r}$ yields the probability of finding the $i$ th electron in the volume of space defined by $d \vec{r}$. Spatial orbitals are both orthonormal,

$$
\int \phi_{i}(\vec{r}) \phi_{j}(\vec{r}) d \vec{r}=\delta_{i j}
$$


and normalized,

$$
\int \phi_{i}(\vec{r}) \phi_{i}(\vec{r}) d \vec{r}=1
$$

In order for the set of orbitals to be complete, they would need to span an infinite range. In reality, however, an infinite expansion is unrealistic and will always be truncated at some value, $M$, such that the set of spatial orbitals becomes $\left\{\phi_{i} \mid i=1,2, \ldots, M\right\}$.

Once again, spatial orbitals depend only on $\vec{r}$ and neglect spin. Introducing a spin coordinate, $\omega$, to define the spin up operator as $\alpha(\omega)$ and a spin down operator as $\beta(\omega)$, a set of $2 M$ spin orbitals, $\phi_{i}$, can be introduced such that

$$
\left.\begin{array}{rl}
\phi_{2 i-1}(\vec{x}) & =\phi_{i}(\vec{r}) \alpha(\omega) \\
\phi_{2 i}(\vec{x}) & =\phi_{i}(\vec{r}) \beta(\omega)
\end{array}\right\} i=1,2, \ldots, M .
$$

This set of spin orbitals also satisfy the requirement of orthonormality and normalization, as defined for the spatial orbitals in Eqs. (2.12) and (2.13).

\subsubsection{Hartree Product}

A full approximate solution to the electronic wavefunction can be simply derived with the application of one assumption: each of the $N$ electrons is non-interacting. The Hamiltonian for such as system would be defined as

$$
\hat{H}_{e}^{H P}=\sum_{i=1}^{N} \hat{h}_{i}
$$

where $\hat{H}_{e}^{H P}$ gives the full electronic Hamiltonian for a system of noninteracting electrons and $\hat{h}_{i}$ represents the simple, one-electron Hamiltonian giving the kinetic and potential energy for the $i$ th electron, in symbolic form as 


$$
\hat{h}_{i}=\hat{T}_{e}+\hat{V}_{e N}
$$

noting the electron-electron term vanishes given non-interacting electrons. The system is comprised of a set of spin orbitals, $\left\{\phi_{i}\left(\vec{x}_{1}\right), \phi_{j}\left(\vec{x}_{2}\right), \ldots, \phi_{k}\left(\vec{x}_{N}\right)\right\}$, where the total energy equates to the sum of the energies of each independent spin orbital, $\varepsilon$,

$$
E^{H P}=\varepsilon_{i}+\varepsilon_{j}+\ldots+\varepsilon_{k} .
$$

Given the form of the electronic Hamiltonian in Eq. (2.15), the total electronic wavefunction yielding the energy eigenvalues in Eq. (2.17) would be a simple product of the individual spin orbitals for each electron,

$$
\left|\Psi^{H P}\left(\vec{x}_{1}, \vec{x}_{2}, \ldots, \vec{x}_{N}\right)\right\rangle=\left|\phi_{1}\left(\vec{x}_{1}\right) \phi_{2}\left(\vec{x}_{2}\right) \ldots \phi_{k}\left(\vec{x}_{N}\right)\right\rangle
$$

This equation represents the Hartree product, where

$$
\hat{H}_{e}^{H P} \Psi^{H P}=E^{H P} \Psi^{H P} .
$$

One issue arising from the assumption of non-interacting electrons becomes readily apparent from further inspection of Eq. (2.18), in that the problem of electron correlation is completely ignored. The probability of finding electron 1 in the volume element and spin state defined by $d \vec{x}_{1}$ is entirely independent to that of finding electron 2 in $d \vec{x}_{2}$, calculated as $\left|\phi_{1}\left(\vec{x}_{1}\right)\right|^{2} d \overrightarrow{x_{1}}$ and $\left|\phi_{2}\left(\vec{x}_{2}\right)\right|^{2} d \overrightarrow{x_{2}}$ respectively. The total probability of finding electron 1 in $d \vec{x}_{1}$ and electron 2 in $d \vec{x}_{2}$ is simply the product of the individual probabilities, $\left|\phi_{1}\left(\vec{x}_{1}\right)\right|^{2}\left|\phi_{2}\left(\vec{x}_{2}\right)\right|^{2} d \overrightarrow{x_{1}} d \overrightarrow{x_{2}}$.

These probabilities completely disregard the electron-electron Coulomb repulsion, making it less probable that one would find two electrons in 
spatially adjacent volume elements than in two spatially segregated volume elements. An additional limitation of the Hartree product is that it places the restriction on electrons that each must occupy a specific spin orbital, i.e., electron 1 must occupy $\phi_{1}$, which disregards the indistinguishability of electrons. Herein, this model also ignores the requirement of antisymmetry, necessitating that the wavefunction be negated if two identical electrons are switched. In order to account for this final limitation, the Slater determinant was introduced.

\subsubsection{Slater Determinants}

The Hartree product for a simple two-electron system, where the electrons have parallel spin and are therefore indistinguishable, could take on two forms:

$$
\Psi_{12}^{H P}\left(\vec{x}_{1}, \vec{x}_{2}\right)=\phi_{i}\left(\vec{x}_{1}\right) \phi_{j}\left(\vec{x}_{2}\right)
$$

or

$$
\Psi_{12}^{H P}\left(\vec{x}_{1}, \vec{x}_{2}\right)=\phi_{i}\left(\vec{x}_{2}\right) \phi_{j}\left(\vec{x}_{1}\right) .
$$

As stated in the previous section, these two ways of writing the wavefunction distinguish between electron 1 at $\vec{x}_{1}$ and electron 2 at $\vec{x}_{2}$ being in a particular spin orbital, either $\phi_{i}$ or $\phi_{j}$, clearly violating antisymmetry for indistinguishable electrons. Reformulating the Hartree product as a linear combination of Eqs. (2.20) and (2.21), however, as

$$
\Psi\left(\vec{x}_{1}, \vec{x}_{2}\right)=\frac{1}{\sqrt{2}}\left[\phi_{i}\left(\vec{x}_{1}\right) \phi_{j}\left(\vec{x}_{2}\right)-\phi_{i}\left(\vec{x}_{2}\right) \phi_{j}\left(\vec{x}_{1}\right)\right],
$$

with the normalizing $(1 / \sqrt{2})$ pre-factor, yields a fully antisymmetric wavefunction where 


$$
\Psi\left(\vec{x}_{1}, \vec{x}_{2}\right)=-\Psi\left(\vec{x}_{2}, \vec{x}_{1}\right)
$$

Considering the case where $j=k$, this reformulation also clearly satisfies the Pauli exclusion principle, where the probability of finding both electron 1 and electron 2 in the same spin orbital is zero.

Eq. (2.22) can be rewritten in terms of a determinant:

$$
\Psi\left(\vec{x}_{1}, \vec{x}_{2}\right)=\frac{1}{\sqrt{2}}\left|\begin{array}{ll}
\phi_{i}\left(\vec{x}_{1}\right) & \phi_{j}\left(\vec{x}_{1}\right) \\
\phi_{i}\left(\vec{x}_{2}\right) & \phi_{j}\left(\vec{x}_{2}\right)
\end{array}\right|,
$$

which defines the Slater determinant for the two electron system. This determinant can be generalized to a system of $N$-electrons as

$$
\Psi\left(\vec{x}_{1}, \vec{x}_{2}, \ldots, \vec{x}_{N}\right)=\frac{1}{\sqrt{N !}}\left|\begin{array}{cccc}
\phi_{i}\left(\vec{x}_{1}\right) & \phi_{j}\left(\vec{x}_{1}\right) & \ldots & \phi_{k}\left(\vec{x}_{1}\right) \\
\phi_{i}\left(\vec{x}_{2}\right) & \phi_{j}\left(\vec{x}_{2}\right) & \ldots & \phi_{k}\left(\vec{x}_{2}\right) \\
\vdots & \vdots & \ddots & \vdots \\
\phi_{i}\left(\vec{x}_{N}\right) & \phi_{j}\left(\vec{x}_{N}\right) & \ldots & \phi_{k}\left(\vec{x}_{N}\right)
\end{array}\right|
$$

which satisfies the antisymmetric requirement, as interchanging two identical electrons equates to interchanging two rows of the determinant, which would also change its sign. The Pauli exclusion principle is also satisfied, as having two electrons occupying the same spin orbital would be equivalent to two equal rows of the Slater determinant, rendering the determinant zero.

Introducing a shorthand notation for the Slater determinant, Eq. (2.25) can be rewritten in terms of its diagonal elements (in Dirac notation) as

$$
\left|\Psi\left(\vec{x}_{1}, \vec{x}_{2}, \ldots, \vec{x}_{N}\right)\right\rangle=\left|\phi_{i}\left(\vec{x}_{1}\right) \phi_{j}\left(\vec{x}_{2}\right) \ldots \phi_{k}\left(\vec{x}_{N}\right)\right\rangle
$$

or choosing the ordering of spatial/spin coordinates to always be $\vec{x}_{1}, \vec{x}_{2}, \ldots, \vec{x}_{N}$ 


$$
|\Psi\rangle=\left|\phi_{i} \phi_{j} \ldots \phi_{k}\right\rangle
$$

Since the Slater determinant satisfies the antisymmetric requirement of the electronic wavefunction, exchange interactions between two electrons are fully accounted for within its formulation. This formulation, however, does not account for electron correlation, which becomes an important consideration when accounting for the limitations of the Hartree-Fock method outlined in the following section.

\subsection{Hartree-Fock}

The Hartree-Fock (HF) method [90, 91, 92] was introduced in 1928 by Douglas Hartree, with contributions from Vladimir Fock and John Slater made in 1930. It is also known as the mean-field approximation, postulating that each electron experiences a force due to a mean electric field induced by all other electrons. This mean-field dictates how the electrons ultimately arrange themselves in a ground-state configuration. In order to address the problem of electron-electron interactions, the HF method first employs the BO approximation and, therefore, addresses only the problem of the electronic wavefunction as defined by Eq. (2.6), leaving the solution to $\chi(\vec{R})$ for a second, simplified step.

A brief, mathematical description of the method generally follows those outlined in Refs. [86, 87]. ${ }^{3}$ The HF method is concerned with finding a set of spin orbitals yielding the closest approximation to the ground state of the electronic wavefunction, in Slater determinant form as

$$
\left|\Psi_{0}\right\rangle=\left|\phi_{1} \phi_{2} \ldots \phi_{N}\right\rangle
$$

\footnotetext{
${ }^{3}$ Ref. [86], pgs. 53-55, 111-122.
} 
Employing the variational principle, the mathematical details of which can be found in Ref. [86], ${ }^{4}$ it can be shown that the spin orbitals accomplishing this minimization also minimize the expectation value of the electronic energy,

$$
E_{0}=\left\langle\Psi_{0}\left|\hat{H}_{e}\right| \Psi_{0}\right\rangle .
$$

The form of the electronic Hamiltonian, $\hat{H}_{e}$, within the HF method takes on the form

$$
\begin{aligned}
\left\langle\Psi_{0}\left|\hat{H}_{e}\right| \Psi_{0}\right\rangle= & \sum_{a}\left\langle\phi_{a}\left|\hat{h}_{a}\right| \phi_{a}\right\rangle+ \\
& \frac{1}{2} \sum_{a b}\left[\left\langle\phi_{a} \phi_{a}\left|\frac{1}{\left|\vec{r}_{a}-\vec{r}_{b}\right|}\right| \phi_{b} \phi_{b}\right\rangle-\left\langle\phi_{a} \phi_{b}\left|\frac{1}{\left|\vec{r}_{a}-\vec{r}_{b}\right|}\right| \phi_{a} \phi_{b}\right\rangle\right],
\end{aligned}
$$

where the first term represents the one-electron energy expectation value defined by the one-electron Hamiltonian, $\hat{h}_{a}$, as shown in Eq. (2.3), summed over all electrons. The second term is the two-electron term approximating electron-electron interactions. Employing functional variation for Eq. (2.30), with the single constraint that the spin orbitals remain orthogonal, one can determine the equation defining a set of spin orbitals giving the best approximation to the ground state electronic wavefunction $[86,87] .{ }^{5}$ The equation defining each spin orbital in the set is given by

$$
\begin{aligned}
\hat{h}_{1} \phi_{a}\left(\vec{x}_{1}\right) & +\sum_{b \neq a}\left[\int \frac{\left|\phi_{b}\left(\vec{x}_{2}\right)\right|^{2}}{\left|\vec{r}_{1}-\vec{r}_{2}\right|} d \vec{x}_{2}\right] \phi_{a}\left(\vec{x}_{1}\right) \\
& -\sum_{b \neq a}\left[\int \frac{\phi_{b}^{*}\left(\vec{x}_{2}\right) \phi_{a}\left(\vec{x}_{2}\right)}{\left|\vec{r}_{1}-\vec{r}_{2}\right|} d \vec{x}_{2}\right] \phi_{b}\left(\vec{x}_{1}\right)=\varepsilon_{a} \phi_{a}\left(\vec{x}_{1}\right),
\end{aligned}
$$

where $\varepsilon_{a}$ is the single-electron energy.

\footnotetext{
${ }^{4}$ Pgs. 115-119.

${ }^{5}$ Ref. [86], pgs. 115-119.
} 
The first term contains the simple, single-electron Hamiltonian for only the kinetic energy and nuclear-electron Coulomb term for electron 1 at $\vec{x}_{1}$, defining the energy of electron 1 in a field of positive point charges as if there were no other electrons present. The second and third terms, however, are two-electron terms. The second is deemed the Coulomb term which can be written as an operator, $\hat{J}$, which for spin orbital $\phi_{a}\left(\vec{x}_{1}\right)$ would be defined as

$$
\hat{J}_{b}\left(\vec{x}_{1}\right) \phi_{a}\left(\vec{x}_{1}\right)=\left[\int \frac{\left|\phi_{b}\left(\vec{x}_{2}\right)\right|^{2}}{\left|\vec{r}_{1}-\vec{r}_{2}\right|} d \vec{x}_{2}\right] \phi_{a}\left(\vec{x}_{1}\right)
$$

While the Coulomb term is, in theory, a two-electron term, it actually represents one electron experiencing an average potential due to a second electron. The potential due to electron 2 at $\vec{x}_{2}$ is averaged over all space in taking the integral, which is then applied to electron 1 at $\vec{x}_{1}$.

The final term in Eq. (2.31), deemed the exchange term, arises from the antisymmetry of the wavefunction and can also take on an operator form, $\hat{K}$, which is defined in how it operates on a given spin orbital:

$$
\hat{K}_{b}\left(\vec{x}_{1}\right) \phi_{a}\left(\vec{x}_{1}\right)=\left[\int \frac{\phi_{b}^{*}\left(\vec{x}_{2}\right) \phi_{a}\left(\vec{x}_{2}\right)}{\left|\vec{r}_{1}-\vec{r}_{2}\right|} d \vec{x}_{2}\right] \phi_{b}\left(\vec{x}_{1}\right),
$$

where $\hat{K}_{b}$ operating on $\phi_{a}\left(\vec{x}_{1}\right)$ yields an expression in terms of $\phi_{b}\left(\vec{x}_{1}\right)$. Employing these two operators, Eq. (2.31) can now be rewritten in eigenvalue form as

$$
\left[\hat{h}_{1}+\sum_{b \neq a} \hat{J}_{b}\left(\vec{x}_{1}\right)-\sum_{b \neq a} \hat{K}_{b}\left(\vec{x}_{1}\right)\right] \phi_{a}\left(\vec{x}_{1}\right)=\varepsilon_{a} \phi_{a}\left(\vec{x}_{1}\right) .
$$

This previous equation defines the energy for a single spin orbital, $\phi_{a}$, which can be generalized to all spin orbitals, $a=1,2, \ldots, N$. Given the restricted summation limit of $b \neq a$, this set of equations will change for 
each new spin orbital making it difficult to generalize. Looking back to Eqs. (2.32) and (2.33), a trick can be introduced in that

$$
\begin{aligned}
\hat{J}_{a}\left(\vec{x}_{1}\right) & \phi_{a}\left(\vec{x}_{1}\right)-\hat{K}_{a}\left(\vec{x}_{1}\right) \phi_{a}\left(\vec{x}_{1}\right) \\
& =\left[\int \frac{\left|\phi_{a}\left(\vec{x}_{2}\right)\right|^{2}}{\left|\vec{r}_{1}-\vec{r}_{2}\right|} d \vec{x}_{2}\right] \phi_{a}\left(\vec{x}_{1}\right)-\left[\int \frac{\phi_{a}^{*}\left(\vec{x}_{2}\right) \phi_{a}\left(\vec{x}_{2}\right)}{\left|\vec{r}_{1}-\vec{r}_{2}\right|} d \vec{x}_{2}\right] \phi_{a}\left(\vec{x}_{1}\right) \\
& =0 .
\end{aligned}
$$

The restriction on the sum can then be dropped and Eq. (2.31) is generalized according to

$$
\left[\hat{h}_{1}+\sum_{b}\left(\hat{J}_{b}\left(\vec{x}_{1}\right)-\hat{K}_{b}\left(\vec{x}_{1}\right)\right)\right] \phi_{a}\left(\vec{x}_{1}\right)=\varepsilon_{a} \phi_{a}\left(\vec{x}_{1}\right) .
$$

Shifting to Dirac representation and equating the operators within the large brackets to the single operator $\hat{f}$, this equation becomes

$$
\hat{f}\left|\phi_{a}\left(\vec{x}_{1}\right)\right\rangle=\varepsilon_{a}\left|\phi_{a}\left(\vec{x}_{1}\right)\right\rangle
$$

where $\hat{f}$ defines the Fock operator and Eq. (2.37) is the Hartree Fock equation. This calculation is repeated for each of the $N$ spin orbitals represented within the original Slater determinant to yield a new set of spin orbitals approximating the electronic ground state of the system. It is interesting to note that the term within the interior brackets of Eq. (2.36), containing the coulomb and exchange operators, defines a potential operator which yields the mean-field that each electron experiences in the presence of the (N-1) other electrons. This Hartree potential gives the HF method its mean-field designation.

In practice, the HF method is an iterative method. An initial guess is made for the spin orbitals comprising the original Slater determinant. The steps outlined above are then taken in order to generate a new set of spin 
orbitals. With each iteration, the Hartree potential is compared with the previous potential. The iterations are stopped when the potential stops changing, or when the mean-field defined by each new set of spin orbitals becomes self-consistent. For this reason, the HF method is sometimes also referred to as the self-consistent field (SCF) method.

Overall, the HF method yields a good approximation to the electronic ground state of a system. There is, however, one significant weakness: while HF theory addresses the issue of exchange exactly, it does not account for electron correlation. This contributes to a sizable error when calculating the total electronic energy. In order to better account for electron correlation energy, a number of methods have been developed to extend HF theory. These methods are branded post-HF methods as they use the HF single Slater determinant as a starting point. Configuration interaction, coupled-cluster and Møller-Plesset perturbation theory improve upon HF by adding increasing levels of excitations to the ground state Slater determinant. Method such as multi-reference configuration interaction and multi-configurational self-consistent field make use of additional determinants to both include excitations as well as broaden or reorganize the wavefunction. A detailed description and mathematical development for each of these methods can be found in Refs. [86, 93], but on a general level, each method builds on the HF formulation in order to more accurately (but still approximately) account for electron correlation effects.

Each of these post-HF methods shares the additional burden of incredibly poor computational scaling with the size of the system, specifically related to the number of basis functions. As an example, for a system with $N$ basis functions, simple HF scales as $N^{4}$, while CCSDT (a version of the coupled-cluster algorithm accounting for single, double and triple excitations) scales as $N^{8}$ [93]. ${ }^{6}$ A lot of work has been invested in order to improve the efficiency of the post-HF methods. Linear scaling approaches, such as density-fitting [94] and local approximations [95], considerably reduce the scaling with respect to basis set size. As correlation effects are 
generally short-range, methods employing linear scaling, such as local coupled cluster with single and double and perturbative triplet excitations and density-fitting (DF-LCCSD(T)) and local second-order Møller-Plesset with density-fitting (DF-LMP2), should be as accurate as each corresponding non-linear scaled method.

Even with linear scaling approaches, each of the post-HF methods listed above becomes computationally intractable very quickly for anything larger than a handful of atoms or several molecules. Density functional theory (DFT), as discussed in the following section, is a method that efficiently calculates the combined exchange and correlation energies. Although this method is not systematically improvable, it can be applied to systems up to thousands of atoms. The method of increments, introduced in Section 2.5 , is an algorithm that extends the electron correlation accuracy of postHF methods to systems that would have been previously rendered computationally unmanageable due to their size or complexity.

\subsection{Density Functional Theory}

Density functional theory (DFT) is a quantum mechanical theory used to describe the ground-state properties of many-body systems. Very generally, DFT uses the particle density as the fundamental variable, which depends on only three spatial variables, as opposed to quantum chemistry ab initio methods using many-body wavefunctions, which depend on significantly greater numbers of variables. One of the greatest benefits of this difference is that it renders DFT computationally tractable for molecules and interacting systems up to thousands of atoms.

DFT has a long history dating back to the Thomas-Fermi model developed in the late 1920's, which assumed that the motions of electrons in an atom are uncorrelated and that their kinetic energy can be approximated by a local approximation of the density of free electrons $[96,97]$. DFT for-

\footnotetext{
${ }^{6}$ Pg. 795.
} 
malism has been covered in great depth in a number of references [98, 99] and will be only briefly revisited here. The basics of the theory are rooted in work completed by Pierre Hohenberg and Walter Kohn in 1964 [100] showing that the ground-state energy of an interacting electron gas (i.e., in the absence of nuclei) could be captured by a universal functional of density added to the traditional equation for energy

$$
E_{0}=\int V_{\text {ext }}(\vec{r}) n_{0}(\vec{r}) d \vec{r}+F\left[n_{0}\right]
$$

where $n_{0}(\vec{r})$ is the non-uniform ground state electron density and $V_{\text {ext }}(\vec{r})$ is the external potential. $F\left[n_{0}\right]$ is the density functional addition, given as

$$
F\left[n_{0}\right]=\left\langle\Psi_{0}\left|\hat{T}_{e}+\hat{V}_{e e}\right| \Psi_{0}\right\rangle
$$

A year after the groundbreaking work of Hohenberg and Kohn, Kohn and Lu Jeu Sham laid the theoretical foundation for what is now known as the Kohn-Sham (KS) DFT [101, 102]. KS introduced a different separation which greatly simplified the task of finding good approximations for the energy functional. Generally, this theoretical framework maps a set of interacting electrons in a real potential into a set of non-interacting, fictitious particles in an effective potential. The KS representation for the energy of a system of electrons of density $n$ is given as

$$
E_{K S}\left[\left\{\phi_{i}\right\}\right]=T_{0}\left[\left\{\phi_{i}\right\}\right]+\int\left(V_{e x t}(\vec{r})+\frac{1}{2} V_{H}(\vec{r})\right) n(\vec{r}) d \vec{r}+E_{x c}[n],
$$

where $\left\{\phi_{i}\right\}$ represent a set of orthonormal orbitals, known as the KS orbitals, and $T_{0}$ is the kinetic energy in the absence of electron-electron interactions. The density of electrons $n(\vec{r})$, is related to the KS orbitals as

$$
n(\vec{r})=\sum_{i}^{\text {occ }} f_{i}\left|\phi_{i}(\vec{r})\right|^{2}
$$


with $f_{i}$ representing the integer occupation numbers. $V_{\text {ext }}(\vec{r})$ is the external potential created by ions located at positions $\vec{R}$, such that

$$
V_{e x t}(\vec{r})=\sum_{I} \frac{Z_{I}}{\left|\vec{R}_{I}-\vec{r}\right|}+\sum_{I<J} \frac{Z_{I} Z_{J}}{\left|\vec{R}_{I}-\vec{R}_{J}\right|}
$$

with the atomic number and position of the $I$ th ion, $Z_{I}$ and $R_{I}$ respectively. $V_{H}$ in Eq. (2.40) represents the Hartree potential, or Coulomb potential for electrons, related to density as

$$
V_{H}(\vec{r})=\int \frac{n\left(\overrightarrow{r^{\prime}}\right)}{\left|\vec{r}-\overrightarrow{r^{\prime}}\right|} d \overrightarrow{r^{\prime}}
$$

The final term in Eq. (2.40), $E_{x c}$, defines the exchange-correlation energy which acts to summarize the electronic exchange and correlation effects (energy) not accounted for in the previous three terms. This exchangecorrelation energy generally consists of two contributions: one from the "exchange" interaction resulting from the Pauli exclusion principle stating that no two electrons can occupy the same quantum state; and the other from "correlation" contributions arising from many electrons within a quantum system interacting with one another.

Noting that Eq. (2.40) is only a function of the KS orbitals, which relate directly to the density depending only on the three spatial coordinates, the KS approximation represents an exceptional simplification from the many-body wavefunction methods. The benefit of the KS approximation is that all terms aside from $E_{x c}$ can be treated exactly. The ground state energy of a system is determined by varying the energy functional shown in Eq. 2.40 and determining its minimum value. In theory, DFT is an exact solution to the problem of determining the ground state energy of any system. In reality, however, the exchange-correlation energy is only known exactly for the free electron gas, rendering DFT an approximate formulation when used to describe any other system. 
The exchange-correlation functionals, $E_{x c}[n]$, take on many forms. Each exchange-correlation functional has both strengths and weaknesses and is generally selected based on the type of problems being addressed. The local density approximation (LDA) is one of the simplest functionals, originally proposed by KS [101] but later improved upon by Vosko, Wilk, and Nusair leading to the commonly used 'VWN' representation [103]. Representing the electron exchange-correlation interactions as a function only of the density at a point local to $\vec{r}$ as

$$
E_{x c}^{\mathrm{LDA}}[n]=\int n(\vec{r}) \varepsilon_{x c}^{\mathrm{LDA}}(n(\vec{r})) d \vec{r}
$$

where $\varepsilon_{x c}^{\mathrm{LDA}}(n(\vec{r}))$ is the LDA exchange-correlation functional. In another class of functionals, known as the generalized gradient approximation (GGA) functionals, the gradient of the electron density, $\nabla n(\vec{r})$, is included such that

$$
E_{x c}^{\mathrm{GGA}}[n]=\int n(\vec{r}) \varepsilon_{x c}^{\mathrm{GGA}}(n(\vec{r}), \nabla n(\vec{r})) d \vec{r} .
$$

Examples of GGA functionals include Perdew-Wang-91 (PW91) [104], PerdewBurke-Ernzerhof (PBE) [105] and Becke-Lee-Yang-Parr (BLYP) [106, 107] functionals. In order to include the exact exchange energy calculated by $\mathrm{HF}$, a class of hybrid functionals were developed that include a linear combination of the HF exchange energy and any number of GGA or LDA functionals. A common example is the Becke, three-parameter, Lee-Yang-Parr (B3LYP) functional [108], given as

$$
\begin{aligned}
E_{x c}^{\mathrm{B} 3 \mathrm{LYP}}[n]=E_{x c}^{\mathrm{LDA}}[n]+ & 0.2\left(E_{x}^{\mathrm{HF}}-E_{x}^{\mathrm{LDA}}\right)+ \\
& 0.72 \Delta E_{x}^{\mathrm{B} 88}+0.81 \Delta E_{c}^{\mathrm{PW} 91} .
\end{aligned}
$$

$E_{x}^{\mathrm{HF}}$ is the exact HF exchange energy and $E_{x}^{\mathrm{LDA}}$ is the exchange-only (minus correlation) portion of the LDA functional [103]. $\Delta E_{x}^{\mathrm{B} 88}$ is Becke's 
gradient correction for exchange [106] and $\Delta E_{c}^{\mathrm{PW} 91}$ is the Perdew-Wang gradient correction for correlation [104].

The advantages of DFT are many. In quantum mechanically addressing electron-electron interactions, it represents a significant improvement over empirical potentials. DFT is also an improvement to the HF method, in that it considers (albeit, approximately) both exchange and correlation interactions for electrons. As a method that scales as $N^{3}$, DFT also represents a computationally efficient method that can be applied to larger physical systems, including up to thousands of atoms or bulk. Additionally, DFT can quite accurately predict the ground state properties of metals, semi-conductors and insulators with the use of an appropriate exchange-correlation functional [109].

While DFT is a powerful tool, there are several disadvantages. Although the exchange-correlation functionals developed to date are quite robust and can be used for a wide range of systems, they still represent only approximations to the exact exchange-correlation energy. Additionally, it is impossible to predict the size of the errors introduced by the exchange-correlation approximations and the method cannot be systematically improved. It has been previously shown that DFT has serious problems calculating properties for weakly interacting systems, such as molecular solids or van der Waals bound crystals [110, 111, 112]. Finally, DFT can only be used to calculate the ground state properties of a system, rendering it useless for systems where excitations dictate properties or behavior. Although still excluding excitations, the finite temperature behavior of a system can be approximated through a DFT-based molecular dynamics modification of the Born-Oppenheimer approximation (introduced in Sec. 2.1), as outlined in the following section. 


\subsubsection{Born Oppenheimer Molecular Dynamics}

Since DFT calculates only the ground state electron density, finite temperature calculations are not directly accessible by the method. However, in applying the Born-Oppenheimer (BO) approximation to molecular dynamics (MD) simulations, one can approximate finite temperature behavior. Generally speaking, separating the solutions to the electronic and ionic wavefunctions allows for the DFT (ground state) calculation of the electron density followed by a finite temperature calculation for the motions of the ions. This section provides an overview of $\mathrm{BO}$ molecular dynamics (BO-MD).

In the classical sense, molecular dynamics calculations consider atoms, including both ionic cores and electrons, as point masses which are moved time-stepwise according to systems of equations governed by a single Hamiltonian. The density-functional (or first-principles) molecular dynamics problem, however, involves a far more complex system involving two sets of particles with a large mass discrepancy: cumbersome ionic cores whose mass is typically $\sim 10^{3}-10^{5}$ times larger than the electrons surrounding them. Thus returning to the Born-Oppenheimer approximation, as outlined in Section 2.1, BO-MD separates the solution to the electronic and ionic Schrödinger equations by assuming the electrons and the ions have coupled but distinct motion.

As a first step in BO-MD, the electronic wavefunction (Eq. (2.6)) is solved using DFT, under the assumption that the ionic cores remain fixed and the less-massive electrons settle instantaneously into their groundstate configuration. The ground-state electron density yields a modified effective electronic potential (Eq. (2.7)), by which the positions of the ionic cores are then adjusted in the second step. In the traditional $\mathrm{BO}$ algorithm, the ionic cores are updated according to the time-dependent ionic Schrödinger equation 


$$
\left[T_{N}+V_{N N}(\vec{R})+\varepsilon_{e}^{\mathrm{eff}}(\vec{R})\right] \chi(\overrightarrow{R, t})=i \hbar \frac{\partial}{\partial t} \chi(\overrightarrow{R, t}) .
$$

Finite temperature is introduced here, in the nuclear kinetic energy term $T_{N}$.

Vienna Ab Initio Software Package (VASP) [113, 114, 115, 116], utilized in this work for all first-principles molecular dynamics calculations, modifies this second step in using classical Newtonian equations of motion to calculate the new ionic positions. In brief summary, the equations start with the classically familiar equation for force,

$$
\begin{aligned}
\frac{\partial P_{I}}{\partial t} & =F_{I} \\
& =-\nabla_{I} E_{0}(\vec{R})
\end{aligned}
$$

where

$$
E_{0}(\vec{R})=\varepsilon_{e}^{\mathrm{eff}}(\vec{R})+V_{N N}(\vec{R})
$$

contains contributions from both the ground state electron densities in the effective electronic field, $\varepsilon_{e}^{\mathrm{eff}}(\vec{R})$, as well as the ion-ion interactions. The force calculation involves the gradient of the effective electronic field, which by employing the Hellmann-Feynman theorem [117, 118], can be simplified to the expectation value of the gradient of the electronic Hamiltonian of the ground state electron density. In Dirac's bra-ket notation, this quantity becomes

$$
\begin{aligned}
-\nabla_{I} \varepsilon_{e}^{\mathrm{eff}}(\vec{R}) & =\nabla_{I}\left\langle\Psi_{0}\left|H_{e}(\vec{R})\right| \Psi_{0}\right\rangle \\
& =\left\langle\nabla_{I} \Psi_{0}\left|H_{e}(\vec{R})\right| \Psi_{0}\right\rangle+\left\langle\Psi_{0}\left|\nabla_{I} H_{e}(\vec{R})\right| \Psi_{0}\right\rangle+\left\langle\Psi_{0}\left|H_{e}(\vec{R})\right| \nabla_{I} \Psi_{0}\right\rangle \\
& =E_{e}(\vec{R})\left\langle\nabla_{I} \Psi_{0} \mid \Psi_{0}\right\rangle+\left\langle\Psi_{0}\left|\nabla_{I} H_{e}(\vec{R})\right| \Psi_{0}\right\rangle+E_{e}(\vec{R})\left\langle\Psi_{0} \mid \nabla_{I} \Psi_{0}\right\rangle \\
& =\left\langle\Psi_{0}\left|\nabla_{I} H_{e}(\vec{R})\right| \Psi_{0}\right\rangle
\end{aligned}
$$


The beauty in this step is that it allows us to calculate the electronic forces on the ions as if the electrons were stationary.

Once the forces on each ionic core are calculated, the new positions of the ions are determined using the velocity Verlet algorithm [119]. Starting with the position, velocity and acceleration from the previous time step, respectively $\vec{x}(t), \vec{v}(t)$ and $\vec{a}(t)$, the half-step (or leap-frog) ionic velocities and new positions can be calculated as

$$
\begin{gathered}
\vec{v}\left(t+\frac{1}{2} \Delta t\right)=\vec{v}(t)+\frac{1}{2} \vec{a}(t) \Delta t \\
\vec{x}(t+\Delta t)=\vec{x}(t)+\vec{v}\left(t+\frac{1}{2} \Delta t\right) \Delta t,
\end{gathered}
$$

where $\vec{x}(t+\Delta t)$ yields the updated position of the ions at the next time step. Using

$$
\vec{a}(t+\Delta t)=\frac{\vec{F}(t+\Delta t)}{m}
$$

where $\vec{F}(t+\Delta t)$ are updated via the Hellman-Feynman theorem using the effective electronic field for the updated time step, we then calculate the new velocities of the ions as

$$
\vec{v}(t+\Delta t)=\vec{v}\left(t+\frac{1}{2} \Delta t\right)+\frac{1}{2} \vec{a}(t+\Delta t) \Delta t
$$

By combining DFT with Newtonian mechanics, VASP's implementation BO approximation renders molecular dynamics trajectories for systems up to thousands of atoms, as well as bulk, computationally tractable and relatively accurate. For the small systems included in this research, this computationally efficient algorithm has allowed the calculation of millions of time steps for multiple cluster sizes at finite temperatures. Although the BO-MD implementation of DFT is an incredibly powerful tool, 
it still faces the limitations outlined in the previous section. The method of increments, introduced in the next section, is an algorithm that can be used to predict the properties of a wide range of systems and overcomes several of the limitations of DFT, in that it is a systematically improvable method which can be applied to weakly interacting systems.

\subsection{Method of Increments}

The method of increments (MI) was introduced by Hermann Stoll in 1992, initially presented as a method extending SCF calculations for electron correlation (HF and post-HF) to crystalline solids and graphite layers [120, $121,122]$. Generally speaking, the method of increments uses a divideand-conquer approach to electron correlation: taking a larger system that would normally be computationally intractable for typical post-HF methods and dividing it up into a set of smaller components, utilizing post$\mathrm{HF}$ algorithms to calculate the electron correlation energies for the smaller pieces and then summing them to give a more accurate total correlation energy. The justification for the method lies in the assumption that the motion of one electron has only a very localized effect on the surrounding electrons, so correlation energies for the smaller components remain accurate even though the calculations ignore the correlation effects on neighboring components. In this way, the method of increments is termed a local correlation method.

An excellent, in-depth review of MI can be found in Refs. [123] and [124], while only a general overview as the method applies to bulk periodic systems will be included here. As mentioned in the previous section, HF exactly accounts for electron exchange effects but does not account for correlation. MI is a method that yields an approximation to the correlation energy.

While MI yields correlation energies at a post-HF level of accuracy, the method is ultimately concerned with providing an estimate of the total 
cohesive energy of a system, from which most ground state properties can be derived. For a solid, this cohesive energy is experimentally calculated per "subunit" of the solid, which is usually an atom or molecule. For MI calculations, this total cohesive energy is given as

$$
E_{\mathrm{coh}}=E_{\mathrm{HF}}+E_{\mathrm{corr}}
$$

where $E_{\text {coh }}$ gives the total cohesive energy per subunit and $E_{\mathrm{HF}}$ gives the total HF energy also calculated per subunit. The final term, $E_{\text {corr }}$, represents the electron correlation energy.

An overview of MI begins with the HF formulation given in terms of localized spin orbitals, where the Slater determinant from Eq. 2.28 is now expressed in terms of these localized orbitals, each centered on a subunit of the crystal. This subunit can consist of a single atom (in atomic crystals), a bond (in graphene, for example) or a molecule (for molecular crystals), depending on the system being studied. With localization, MI then breaks the periodic system down into different sized, smaller groups of localized orbitals and calculates the contribution to the total correlation energy arising from each group using one of the more accurate post-HF methods coupled with localization (typically local coupled-cluster or local Møller-Plesset perturbation theory). The simplest MI group is the onecenter increment, $\epsilon_{i}$, given as

$$
\epsilon_{i}=E_{i}-E_{\mathrm{HF}}
$$

which is comprised of a single subunit of the solid. For the sake of simplicity, this will be assumed to be the atomic subunit in subsequent discussion. The energies $E_{i}$ and $E_{\mathrm{HF}}$ can represent different entities based on the type of system being modeled and the orbital group, $i$, that is chosen. One logical choice for the orbital group of an atomic crystal would be all localized orbitals centered on one atom. $E_{\mathrm{HF}}$ would then represent the total 
canonical HF energy of the single atom and $E_{i}$ would be the energy of that same atom calculated with one of the local post-HF methods. As post-HF methods use the HF single determinant as a starting point, then add perturbations or excitations in order to better account for electron correlation energy arising from opposite-spin electrons, subtracting the total HF energy from the total post-HF energy gives the correlation energy contribution calculated by the more accurate post-HF method. For the one-center increment, one additional consideration must be made in order to account for errors arising from the basis set superposition error (BSSE) of the correlated molecule. A counterpoise calculation is completed that treats the correlated center as a 'ghost-atom' by placing only the appropriate basis set in its location. Eq. (2.57) is then modified as

$$
\epsilon_{i}=E_{i}-E_{C P}-E_{\mathrm{HF}}
$$

where $E_{C P}$ represents the counterpoise energy of the correlated center.

While the above description represents one choice of localization, more frequently the calculations are performed using clusters of atoms - the central atom and its nearest-neighbors, for example. In this model, the cluster is isolated from the remainder of the bulk system and the canonical $\mathrm{HF}$ energy is calculated for the cluster, yielding $E_{\mathrm{HF}}$. The orbitals for the cluster are then localized. The post-HF energy is calculated for only the central atom's localized orbitals, while the rest of the cluster is assumed to be in a 'frozen Hartree Fock' state, once again yielding only the correlation contribution from the local post-HF calculation, $\epsilon_{i}$. Since MI is interested in the total correlation energy per unit cell, if there are $n$ atoms in the unit cell, this calculation will be performed $n$ times and then summed for a total one-center correlation contribution.

The one-center increment, by itself, is typically a poor estimate of the total correlation energy. It is, therefore, often necessary to also consider contributions from higher-level increments: two-center, three-center, etc.. 
Using the cluster model listed above, the two-center scheme would start with a cluster consisting of two atoms separated by some distance and all the nearest-neighbors (or other method to define the cluster) for each atom. If the center atoms are closely spaced, many of the nearest-neighbors will overlap. If, however, the atoms are separated by some distance, the two sets of nearest neighbors will be entirely distinct.

Generally, the same steps are followed as in the one-center case: the canonical HF energy is calculated for the entire cluster; the orbitals for the cluster are localized and the post-HF energy is calculated for the set of localized orbitals belonging to both central atoms, assuming the remainder of the cluster is in a frozen HF state; $E_{\mathrm{HF}}$ is then subtracted from the total energy of the two-center calculation, $E_{i j}$. For the two-center and higher calculations, however, there are two additional considerations. First, the two-center calculation contains correlation contributions from each of the one-centers that comprise it. In order for the two-center increment to represent only the two-center correlation contribution, the one-center increment must be calculated for each center atom - now treating the second central atom as if it were part of the frozen-HF background - and then subtracted from the total energy. The incremental energies for the two- and three-centers are then calculated as

$$
\epsilon_{i j}=E_{i j}-E_{\mathrm{HF}}-\epsilon_{i}-\epsilon_{j}
$$

and

$$
\epsilon_{i j k}=E_{i j k}-E_{\mathrm{HF}}-\left(\epsilon_{i j}+\epsilon_{i k}+\epsilon_{j k}\right)-\left(\epsilon_{i}+\epsilon_{j}+\epsilon_{k}\right),
$$

noting that the three-center increment, $\epsilon_{i j k}$, contains a set of three onecenter correlation energies and three combinations of two-center correlation energies. It should be noted that the one-center increments shown in Eq. (2.59) and (2.60) do not include counterpoise corrections, as this is 
automatically accounted in the calculation of the other one-center contributions. For example, BSSE is accounted for with $\epsilon_{i}$ when that center is treated in a frozen-HF manner for the calculation of $\epsilon_{j}$. While four-center and greater increments can also be calculated, it has been shown that the majority of the total correlation energy is captured by the one-, two- and three-center increments for most systems.

The second consideration is that for any periodic crystal, there are an infinite number of two- and three-center combinations contributing to the total correlation energy. This issue is handled by first constraining one of the centers to the unit cell, for reasons to be discussed below. Theoretically, there are still an infinite number of possible locations for the second (and third) center(s); however, in practice, the correlation contribution will become insignificantly small as the distance between the centers is increased. Generally, all combinations for the second and third center location are calculated, constrained to some cut-off distance of the first, unit cell-bound center, and each increment is summed to give the total correlation contribution. The MI correlation energy can now be defined as

$$
E_{\mathrm{corr}}^{M I}=\sum_{i \in U . C .} \epsilon_{i}+\frac{1}{2 !} \sum_{i \in U . C .} \sum_{j \neq i} \epsilon_{i j}+\frac{1}{3 !} \sum_{i \in U . C .} \sum_{j \neq i} \sum_{k \neq j \neq i} \epsilon_{i j k}+\ldots,
$$

where $i$ is constrained to the first unit cell and the indices $\{j, k\}$ run over the entire crystal, up to the cut-off chosen for the two-, three-, etc. incremental scheme. The summation pre-factors of $1 / N$ ! relate back to the unit cell constraint. It is easiest to see when one considers a one-atom unit cell. For the two-center case, only half of the correlation energy calculated for the two-center increment contributes to the unit cell. For the three-center calculation, a pre-factor of $1 / 3$ comes from the fact that only 1 out of 3 atoms are within the unit cell. The remaining $1 / 2$ pre-factor comes from the constraints on the sums, where each of the combinations for the other two atoms will be summed twice. For a solid, experimental energies are typically given per unit cell so in order to ease comparisons, MI uses the 
same metric.

MI is a powerful tool that allows the calculation of accurate, post-HF correlation energies in a systematically improvable way, for systems that would have previously been computationally intractable. While post-HF methods are also systematically improvable (in adding more perturbation or excitation terms), each improvement dramatically increases their computational scaling making these algorithms only reasonable for smaller and smaller systems. MI, on the other hand, has now been applied to a large number of systems from bulk to surfaces. While most studies have found that one-, two-and three-center increments account for the majority of the correlation energy, the method can easily be extended to more increments if necessary.

In this way, unlike DFT, MI is a systematically improvable method for calculating the correlation energy of a wide range of systems and is wellsuited to capturing the nature of bonding in the solid halogens. Like all methods, however, MI has limitations. One such limitation is that given its employment of post-HF methods, it would become computationally cumbersome to calculate the properties of a larger system over many time steps, as would be required for the thermodynamic modeling of gallium clusters. Initial testing for the small gallium cluster simulations was, therefore, completed using DFT, as outlined in the following chapter. 


\section{Chapter 3}

\section{DFT for Gallium}

The electron exchange and correlation energies, as described in the previous section, are integral pieces of the total cohesive energy of any system. These energies, therefore, play a key role in defining the atomic, molecular and bulk properties of any systems. As electron-electron interactions also largely define atomic bonding, correlation and exchange factor largely into a system's response to increasing temperature and the nature of phasetransitions. In order to properly address how small gallium clusters melt, it was necessary to test the adequacy of DFT calculations in describing the properties of gallium prior to starting melting simulations. This chapter describes the initial benchmarking of DFT for small gallium clusters, calculating the relative bonding contributions of the different geometric components comprising the 6-atom gallium cluster.

\subsection{Symmetric Octahedron}

Using the NWChem computational quantum chemistry software package [125], a series of DFT calculations were completed in order to quantify the many-body energy contributions to a symmetric, neutral $\mathrm{Ga}_{6}$ octahedron. This process entailed calculating dissociation curves for each of the geometric components comprising the octahedron: dimer (singlet and triplet 
states), trimer (isosceles and equilateral), tetramer (square and tetrahedron) and pentamer curves. These calculations were completed with a selection of different basis sets and DFT exchange-correlation functionals in order to test the adequacy of each functional in describing the intricacies of gallium.

Overall, one would generally expect the total energy for the symmetric $\mathrm{Ga}_{6}$ cluster to be described by

$$
\begin{aligned}
E_{T}^{\text {octa }}=E_{6 B} & +6 E_{5 B}+3 E_{4 B}[S q]+8 E_{4 B}[\text { Tet }] \\
& +8 E_{3 B}[E q]+8 E_{3 B}[\text { Isos }]+12 E_{2 B}+6 E_{1 B},
\end{aligned}
$$

where $E_{T}^{\text {octa }}$ represents the total energy for the symmetric octahedron. Each of the $E_{N B}$ terms represent the N-body energy contribution for that size/configuration, while the multiplicative factors represent the number of times those specific geometries are observed in the symmetric octahedron. Each term represents the total energy contribution not described by the preceding terms. The same is true for each of the $N$-body energies. Giving the 5-body energy as an example, the total energy not calculated by the sum of the previous 4, 3, 2 and 1-body term is given as

$$
\begin{aligned}
E_{5 B}=E_{T}^{p e n t} & -E_{4 B}[S q]-4 E_{4 B}[T e t]-4 E_{3 B}[E q] \\
& -6 E_{3 B}[\text { Isos }]-8 E_{2 B}-5 E_{1 B} .
\end{aligned}
$$

Geometrically, the 5-atom pyramid within the symmetric octahedron contains one square, 4 tetrahedra, 4 equilateral triangles, 6 isosceles triangles, 8 dimer bonds and 5 individual atoms.

This calculation can be instructive in that it illustrates the total energy of a structure as fractions of contributions from each of the N-body interactions. For gallium, the interest was particularly focused on the 2-body interactions: given the dimeric nature of bulk gallium, could this dimeric nature be diagnosed in small clusters by measuring the relative percentage 
of 2-body contributions to the total energy. The following sections give an overview of the method as well as a summary of the results.

\subsection{Basis Sets and Functionals}

In setting up the calculations, a variety of parameters were utilized in order to test the differing effects on the calculations. Two basis sets from the EMSL library were tested: aug-cc-PP-VDZ and aug-cc-PP-VTZ [126]. Three DFT exchange-correlation functionals were also utilized: PW-91 [127], PBE [105] and LDA [101, 103]. The calculations employed two different methods for initializing molecular orbital vectors (as initial guesses for each calculation). NWChem offers a standard atomic set of molecular orbital vectors that are created by superimposing the atomic orbital density matrices from a set of neutral atoms. The second initialization method involves the direct calculation of molecular orbital vectors for fragments of the system, then superimposing these output molecular orbital vectors as inputs for the total calculation. Given that NWChem can utilize the molecular orbital vectors from the previous iteration as an initial guess for a current calculation, each dissociation curve and geometry optimization was tested using different initial bond lengths. A summary of the effects of each setting is included in the following section, which details the calculations completed for the gallium dimer.

\subsection{Gallium Dimer}

The investigation began with the gallium dimer using the aug-cc-PP-VDZ basis set. It was first verified that the triplet state was, in fact, the ground state for each of the three functionals. Then, a series of dissociation calculations were completed for the trimer state, using only the PW-91 functional for consistency. A series of tests were completed measuring the impact of atomic versus fragment initialization for the molecular orbital vectors as 
well as various initial bond lengths. All settings aside from these two were maintained between the sets of calculations.

As can be seen in Fig. 3.1, two different states were clearly identified. The ground state calculation was initialized by fragmenting the system into two single gallium atoms, then superimposing the molecular orbital vectors for a starting bond distance of $12 \AA$. Each consecutive calculation decremented the previous bond distance by $0.025 \AA$ and used the molecular orbital vectors for the previous calculation as the initial guess. This state has a minimum at $2.75 \AA$ and dissociates properly to $0 \mathrm{eV}$.

A second calculation was also initialized with a bond separation of 12 Å using the same decrementing scheme for calculations, but used the standard atomic molecular orbital vectors as an initial guess. This excited state has a minimum at $2.475 \AA$ and dissociates to $\sim 0.11 \mathrm{eV}$. A third behavior was observed for a calculation initialized with fragments but starting from a point near the ground state minimum and calculating both increasing and decreasing atomic separations from that point. So that some overlap occurred, one calculation was initialized at a bond distance of $2.6 \AA$ and was decremented using the same method as above. The other calculation started from $2.4 \AA$ with each consecutive calculation incrementing 0.025 $\AA$. This calculation yields the ground state minimum but dissociates to the same $\sim 0.11 \mathrm{eV}$ as the higher-energy state calculation.

Further testing revealed that two similar states were also determined by the PBE and LDA functionals, as summarized in Table 3.1. As expected, the PW91 and PBE functionals yield nearly identical bond lengths as well as energy differences. Although the energy of each state was only $10 \mathrm{meV}$ different for LDA, the shorter bond formed the ground state as opposed to the longer bond for the PW91 and PBE functionals. In agreement with the LDA functional, $\operatorname{CCSD}(\mathrm{T})$ calculations find the longer bond is the ground state for the gallium dimer [49].

Overall, these two states correlate well with bulk gallium's structural parameters where the buckled plane dimers have a bond of $\sim 2.44 \AA$, with 


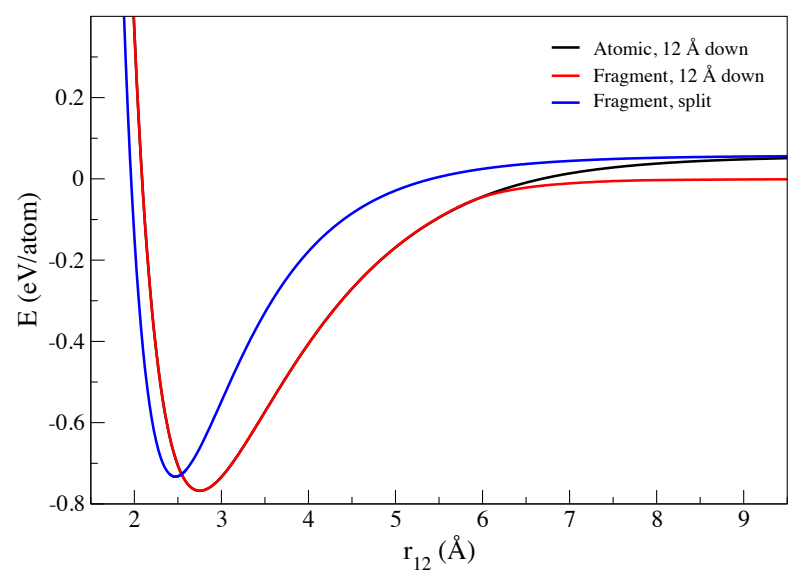

Figure 3.1. The two triplet dimer states determined by dissociation calculations. The ground state (black) was initialized by fragmenting the initial molecular orbital vectors and incrementing down from $r_{12}=12 \AA$. An excited state (red) was initialized with the standard atomic molecular orbitals vectors, also calculating down from $r_{12}=12 \AA$. The third calculation (blue) was initialized by fragmenting the molecular orbitals vectors but split the starting values: one was initialized at $r_{12}=2.6 \AA$ and incremented down; the other at $r_{12}=2.4 \AA$ and incremented up.

the next nearest-neighbors formed by atoms between planes at $\sim 2.71$ $2.80 \AA$ [5]. These calculations are also consistent with previous theoretical work, which has included both DFT [48] and post-HF calculations [49]. The fact that the simple gallium dimer already reveals two states with such closely spaced energies foreshadows the subsequent complexities encountered when calculating the N-body energies.

\subsection{N-body Increments}

For the remaining $\mathrm{N}$-body cohesive energies ( $\mathrm{N}=3,4$ and 5), the complexities seen in the gallium dimer grew more frequent with increasing 


\begin{tabular}{|c||ccc||ccc||ccc||c|}
\hline \multicolumn{1}{|c||}{} & \multicolumn{3}{c||}{ PW91 } & \multicolumn{3}{c||}{ LDA } & \multicolumn{3}{c||}{ PBE } & CCSD(T) \\
& $r_{12}$ & $E$ & $\Delta E$ & $r_{12}$ & $E$ & $\Delta E$ & $r_{12}$ & $E$ & $\Delta E$ & $r_{12}$ \\
\hline$r_{G S}$ & 2.47 & -0.74 & 0.04 & 2.68 & -0.93 & $\star$ & 2.47 & -0.72 & 0.04 & 2.73 \\
\hline$r_{E X}$ & 2.75 & -0.78 & $\star$ & 2.41 & -0.94 & 0.01 & 2.76 & -0.76 & $\star$ & 2.48 \\
\hline
\end{tabular}

Table 3.1. Triplet dimer minimum bond distance and energy comparison of the ground state $\left(r_{G S}\right)$ and the second, low-energy state $\left(r_{E X}\right)$ for PW91, LDA and PBE. Bond distances $\left(r_{12}\right)$ are in angstroms and energies $(E, \Delta E)$ are in $\mathrm{eV} /$ atom. As a point of comparison, $r_{12}$ values obtained by a more accurate $\operatorname{CSSD}(\mathrm{T})$ calculation for the ground state triplet $\left(r_{G S}\right)$ and excited state singlet $\left(r_{E X}\right)$ dimer are also included [49].

cluster size. Although two, generally consistent curves were obtained for the isosceles and equilateral trimers (doublet state) using the double-zeta basis set, neither dissociated to $0 \mathrm{eV}$ /atom and, therefore, did not likely represent the ground states. There were some energy inconsistencies observed at shortest bond lengths for the isosceles trimer, but this did not interfere with the determination of a clear minimum energy configuration. In varying molecular orbital vector initialization and starting geometries, the trimer calculations were quite finicky, often exhibiting energy discontinuities that were correlated with small changes in the spin expectation values, as the calculations progressed. The two consistent curves as well as one example of the energy inconsistencies are illustrated in Fig. 3.2. For the trimer calculations, the fragmented molecular orbital vector calculations were superimposed from a gallium dimer (singlet) and a single gallium atom.

For tetramer and pentamer calculations, no stable dissociation curves were obtained as these calculations were dominated by energy inconsistencies. In an attempt to stabilize the calculations, the triple-zeta basis set was implemented and tested for each of the trimer, tetramer and pentamer configurations. This change introduced some stability for the trimer calcu- 


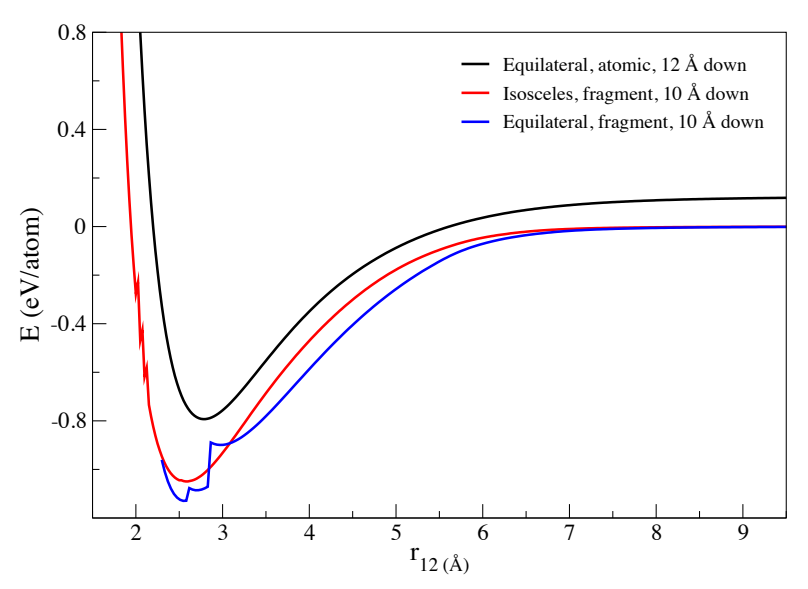

Figure 3.2. Three trimer curves showing: (black) an equilateral calculation initialized with atomic molecular orbital vectors and decremented from 12 $\AA$; (red) an isosceles calculation, initialized with fragmented molecular orbital vectors and decremented from $10 \AA$ (bond distances are given for the single, shorter leg of the triangle); (blue) an equilateral calculation initialized with fragmented molecular orbital vectors and decremented from 10 $\AA$. The discontinuities near the minimum of the blue curve relate to small changes in the spin expectation values.

lations in that a ground state could be identified for the equilateral trimer (where $r_{12}=2.97 \AA$ ). However, frequent energy inconsistencies remained for the isosceles, tetramer and pentamer cases, making a consistent determination of ground state geometries impossible. For this reason, only geometry optimizations were completed for the 6-atom gallium cluster, as outlined in the following section.

\subsection{6-atom Gallium}

In order to determine the global minimum (GM) structure of the 6-atom gallium cluster, a series of DFT geometry optimizations were completed 
using different functionals and initial geometries. In agreement with [48, $49,50]$, the results verified that the "book" structure, as shown in Fig. 3.3, was the lowest energy isomer for each functional. Using PW91 values as the example, the book structure has an energy of $-1.61 \mathrm{eV} /$ atom and forms a slightly asymmetric structure, as the bonds lengths vary between $2.58-2.64 \AA$. It is interesting to note that this ground state $\mathrm{Ga}_{6}$ structure is very dimeric in nature, remnant of the pair bonding observed in bulk $\alpha$-gallium. The next lowest energy isomer was an asymmetric octahedral structure, with the bond from z-axis top and bottom atoms being a full angstrom shorter than the bonds between the xy-plane square atoms, at 2.7 and $3.7 \AA$ respectively. At $-1.21 \mathrm{eV} /$ atom, this structure had significantly higher energy than the book structure.

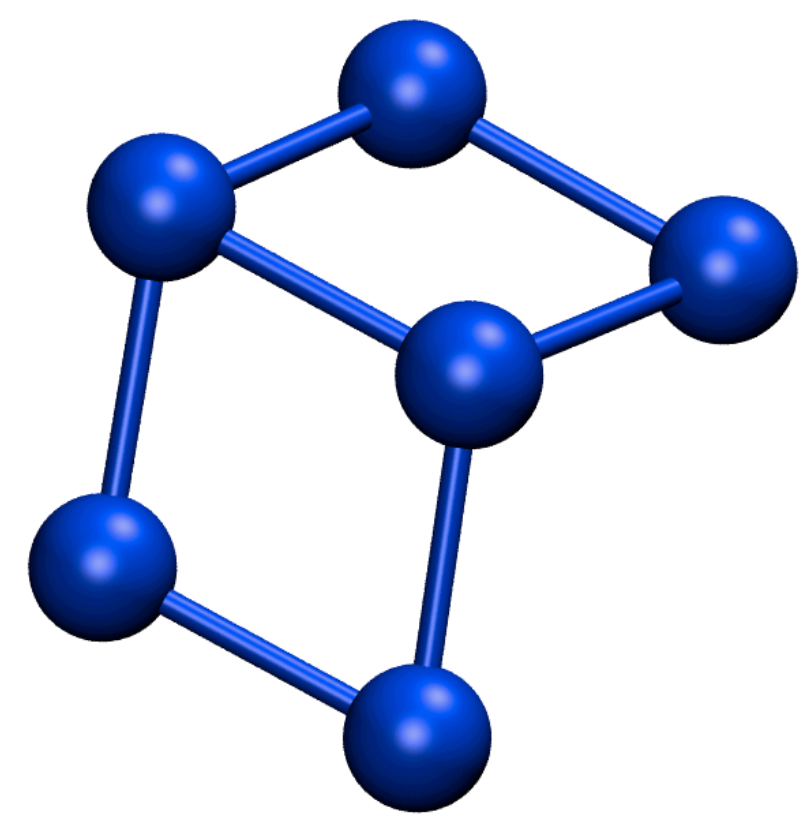

Figure 3.3. The lowest energy isomer for the $\mathrm{Ga}_{6}$ structure, $E_{P W 91}=-1.61$ $\mathrm{eV} /$ atom. Atomic coordinates are given in Tab. B.1.

These results illustrated that DFT-calculated lowest energy bond lengths 
for the gallium dimer are in excellent agreement with bulk values as well as previous DFT [48] and post-HF calculations [49]. Additionally, DFT optimizations find the book structure as the ground state configuration for the 6-atom structure, also in agreement with previous calculations [48, 49, 50]. While the calculation of many-body contributions to the binding energy of small gallium clusters was riddled with energy inconsistencies, the exercise yielded a better appreciation for the rich complexities of the potential energy landscape for small gallium clusters, as has been previously reported by Refs. $[4,49,128]$. This understanding helped guide the choice of initial structures, as well as the implementation of an algorithm aiding the exploration of configurational phase space (parallel tempering). The initial testing and settings for the MD melting simulations are introduced in the following chapter. 


\section{Chapter 4}

\section{Melting}

A set of molecular dynamics (MD) simulations were completed in order to explore the greater-than-bulk melting phenomenon observed for small gallium clusters. This chapter provides the details of the initial structures, MD settings, parallel tempering implementation (to aid with configurational phase space sampling), as well as the analysis techniques utilized in summarizing the data.

\subsection{Initial structures}

Prior to initiating MD, the calculations required a set of gallium cluster structures in the size-range of interest as seeds for the simulations. Ideally, these would be ground state structures; however, given gallium's complex potential energy landscape, the computational time required to determine low-energy gallium structures was assumed to be extensive and that true ground state status would be difficult to ascertain. In order to devote most computational resources to MD simulations, a selection of ground state aluminum structures, given by Ref. [129], served as seeds for determining a set of low-energy, initial gallium structures. These $A l_{n}$ clusters $(n=20$ 34) were derived by an extensive, multi-stage genetic algorithm search. As aluminum shares group-13 status with gallium, and the cluster sizes 
overlapped (in part) with the experimental data for gallium clusters, these clusters provided an ideal set of configurations from which initial gallium cluster structures could be derived.

Given its success in describing the properties of $\mathrm{Ga}_{2}$ and $\mathrm{Ga}_{6}$, as described in the previous chapter, DFT was utilized for determining this set of low-energy initial structures. For each initial $A l_{n}$ structure, densityfunctional theory (DFT) conjugate-gradient optimizations were completed using gallium Projector-Augmented Wave (PAW) potentials [130, 131], as they are generally considered more accurate and reliable than the ultrasoft pseudopotentials [131], with an energy cut-off of $350 \mathrm{eV}$ and the GGAPW91 exchange-correlation functional [127, 132], as implemented in VASP $5.2[113,114,115,116]$. For each of the 15 cluster sizes, the optimizations yielded gallium structures exhibiting strong structural coherence with their aluminum seeds, with the gallium structures being slightly wider than their aluminum counterparts. Figure 4.1 demonstrates a selection of the optimized gallium structures paired with their aluminum seeds.

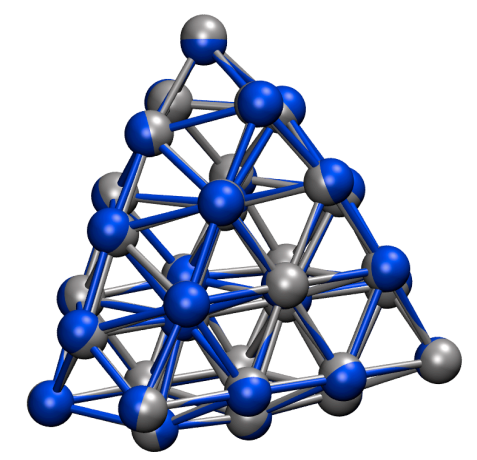

(a)

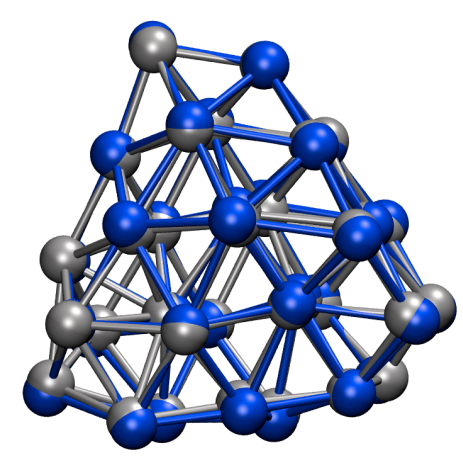

(b)

Figure 4.1. Comparisons of the aluminum seed structures (silver) with the optimized gallium structures (blue) for (a) 32-atom and (b) 33-atom clusters. Atomic coordinates for each structure are given in Tabs. B.2 through B.5. 
Armed with this set of low-energy gallium cluster structures, the calculations progressed to MD simulations. Although it was assumed that DFTbased MD would be necessary to adequately simulate the thermodynamics of these small systems, a set of classical MD simulations first were completed in order to test the adequacy of this simpler, less computationallyintensive model. The following section describes these simulations and provides an overview of the results.

\subsection{Classical MD}

Classical MD simulations were completed using the Large-scale Atomic / Molecular Massively Parallel Simulator (LAMMPS) software package $[133,134]$. The simulations were performed in the canonical ensemble (constant number of particles, volume and temperature) using a NoséHoover thermostat [135] coupled with the GaN Tersoff potential [136]. As the only available potential for gallium within LAMMPS, the Tersoff potential was selected by necessity. However, as a many-body potential with the ability to capture preferred bond-angle orientations as well as interatomic distances, the potential was an appropriate choice for classical MD simulations on small gallium clusters.

Generally speaking, classical MD ignores explicit electron-electron interactions altogether, treating each atom as if it were a point mass including both the ionic cores and electrons as a single body. The equations of motion that govern classical MD simulations consist of coupled velocity and force equations, typically described by Newtonian mechanics. Within LAMMPS, these coupled equations are given as

$$
m_{i} \frac{d \vec{v}_{i}}{d t}=\sum_{j} F_{2}\left(\vec{r}_{i}, \vec{r}_{j}\right)+\sum_{j} \sum_{k} F_{3}\left(\vec{r}_{i}, \vec{r}_{j}, \vec{r}_{k}\right)+\ldots
$$

and 


$$
\frac{d \vec{r}_{i}}{d t}=\vec{v}_{i}
$$

where $m_{i}, v_{i}$ and $r_{i}$ represent the mass, velocity and position of ion $i$, respectively. $F_{2}$ represents the two-body force between ions $i$ and $j$ and $F_{3}$ defines the three-body interactions between ions $i, j$ and $k$. The force terms are defined by the potential energy functional for the system and higher-order forces (between 4 ions, etc.) can also be included depending on the potential. Within this work, the forces were given by the GaN Tersoff potential [136], which is a three-body (truncates at $F_{3}$ ), empirical interatomic potential that can account for the hybrid forces acting between non-similar atoms (such as gallium and nitrogen in GaN). Although this research investigates pure gallium clusters, the GaN potential simply adds a single parameter to a set of previously developed Tersoff potentials for single-element systems [137] and is, therefore, still accurate for non-hybrid materials.

The Nosé-Hoover thermostat is based on Nosé's original formulation for constant temperature simulations, which couples the Newtonian equations of motion to a heat bath in order to maintain a constant average temperature [138]. Nosé's equations required a time-scaling variable that would oscillate as the calculations progressed. Hoover modified these equations by eliminating the need for the time-scaling variable, essentially recasting the thermostatting in terms of a thermodynamic frictional coefficient [135].

The canonical simulations employed in this research took each of the 15 clusters through a heating cycle followed by a cooling cycle, each spanning $100 \mathrm{~K}-1190 \mathrm{~K}$ in $10 \mathrm{~K}$ increments. For all 15 clusters, the results produced irregular, high-frequency oscillations for the canonical specific heat curves and numerous inconsistencies in the potential energy curves, as exemplified by the results for the 31-atom cluster illustrated in Figure 4.2. Although erratic, a melting transition could be identified between $\sim 550-650$ 
$\mathrm{K}$ for each of the 15 clusters, which closely matches the published value of the GaN Tersoff potential's measurement of the melting temperature of $\alpha$-gallium at $600 \pm 100 \mathrm{~K}$ [2]. While some of the irregularity in the calculations could likely be attributed to the small number of atoms per cluster, this bulk melting temperature match for the GaN Tersoff potential was a strong indicator that the exclusion of explicit electron-electron interactions would be an insufficient model to capture the intricate thermodynamic properties of these small systems.

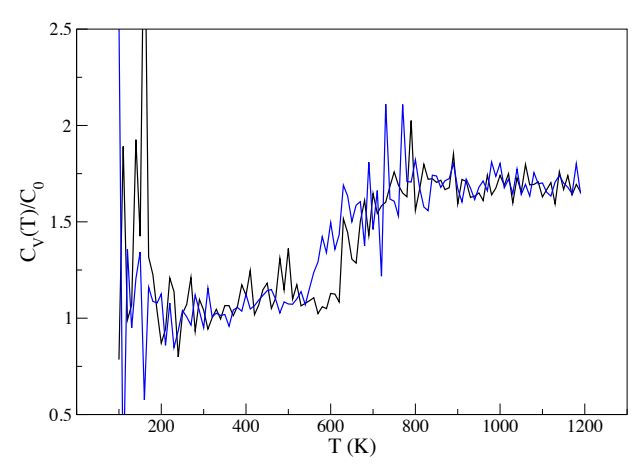

(a)

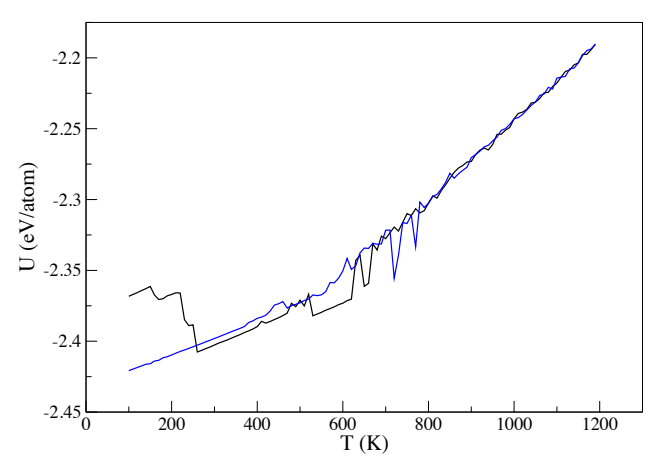

(b)

Figure 4.2. (a) Canonical specific heat and (b) potential energy curves for the heating (black) and cooling (blue) cycles of classical molecular dynamics runs. The curves illustrate the results for $\mathrm{Ga}_{31}$, but are characteristic of the results for all $\mathrm{Ga}_{n}$ clusters $(\mathrm{n}=20-34)$.

One interesting feature consistent between each simulation was the noticeable drop in potential energy at the beginning of each heating cycle, as can be seen on the black curve in Figure 4.2(b). This drop would be characteristic for a structure settling into a lower energy configuration as it gained energy, through heating, to overcome a potential energy barrier within a local minima energy bin. Although this energy difference could have been specific to the Tersoff potential model, it warranted further investigation. For each of the 15 simulations, a structure was extracted at 190 $\mathrm{K}$ during the cooling cycle. This structure was then optimized with VASP 
using the same settings listed in Section 4.1. Most structures optimized to a higher energy than the aluminum-seeded structures; however, the classical MD-derived structures for $\mathrm{Ga}_{20}$ and $\mathrm{Ga}_{28}$ optimized to a lower energy. This $\mathrm{Ga}_{20}$ structure was subsequently used as an input for DFT-based, or first-principles, MD calculations, as described in the following section.

\subsection{First-Principles MD}

Given that the classical MD simulations failed to capture the thermodynamic intricacies of small gallium clusters, more accurate first-principles molecular dynamics (FPMD) were employed. Generally speaking, FPMD considers the movements of the electrons and ions to be distinct. The first-principles designation indicates that DFT is used to calculate the electronic ground state (electron densities) for a given ionic configuration. The molecular dynamics designation means that the ions are updated according to a time-stepwise scheme, similar to the procedure outlined for classical MD but with a different set of equations of motion governing the dynamics. The following sections give an overview of the settings, methods and equations relevant to the FPMD implementation employed for this research.

\subsubsection{Ensemble selection}

Prior to starting simulations, the choice between the canonical and microcanonical ensembles was investigated. VASP's only available thermostat for canonical simulations was the Nosé-Hoover thermostat, outlined in Sec. 4.2. Unfortunately, previous studies have shown that when used with small systems such as clusters, this thermostat often fails to sample configurational phase space in a way that generates the expected distribution of potential energies in the canonical ensemble $[139,140]$. Although previous research attempting to model small gallium cluster melting has employed 
the canonical ensemble $[47,32,45]$, the issue of thermostatting was altogether avoided by scaling the velocities of the ions. This method added several parameters to the model, such as the frequency of rescaling, which can introduce numerical instabilities into the calculations [141]. Wishing to avoid the inadequacies of thermostatting and questions surrounding velocity scaling, the microcanonical ensemble was selected for FPMD calculations completed in this work. The specific settings employed for VASP's implementation of microcanonical FPMD are outlined in the following section.

\subsubsection{VASP Settings}

FPMD simulations were performed using VASP's implementation of BornOppenheimer MD in the microcanonical ensemble. All settings were maintained from the geometry optimizations, as described in Section 4.1, aside from the energy cut-off which was reset to the default. Each simulation included 10 ps of canonical equilibration for 37 temperatures spanning 200$2000 \mathrm{~K}$, enabling the selection of 19 runs that adequately spanned the temperature range of experimental interest with approximately evenly-spaced average energy values. For these 19 simulations, the canonical equilibration was followed by a period of microcanonical relaxation and simulation time. The total simulation time per cluster varied, depending on convergence as determined by quantitative and qualitative measures of change in the specific heat. This measure, as well as other details related to the settings as described below, will be discussed in greater detail in Chapter 5 .

Initial testing was completed to measure the effects of varying time steps on the results. In VASP's treatment of two distinct Hamiltonians within the BO approximation, a small amount of total energy change was observed when the time step was greater than $2.5 \mathrm{fs}$. Therefore, all calculations utilized a time step of $2 \mathrm{fs}$, yielding total energy oscillations on the 
order of $\sim 0.001 \mathrm{eV}$, with negligible $(<0.005 \mathrm{eV})$ total energy drift. For both optimizations and $\mathrm{MD}$, we added corrections for the full dipole moment in all directions in order to treat the cationic structure. Due to periodic boundary conditions within VASP, the code requires that the total charge per unit cell be neutral. For a cluster with a charge of $+e$, VASP adds a background charge of $-e$ to the unit cell. This introduces errors to the potential and forces which need to be accounted for with dipole corrections. These corrections had only minimal effect on the optimized structures, but were determined to be necessary for an accurate description throughout the MD trajectory as they yielded different structures over relatively short simulation times.

To verify the effects of the valence treatment of the $d$-electrons, four FPMD simulations were completed employing two different pseudopotential models for the same cluster size. VASP offers two PAW potentials for gallium: a small-core (SC) model which treats the $d$-electrons as valence, yielding a valence set of $4 s^{2} 3 d^{10} 4 p^{1}$; and a large-core (LC) model treating the $d$-electrons as core, with a valence set of $4 s^{2} 4 p^{1}$. Simulations comparing the SC and LC models for $\mathrm{Ga}_{20}$ and $\mathrm{Ga}_{20}^{+}$reveal that the effect of the $d$-electrons was minimal, as described in greater detail in Chapter 5.

Additional testing for the effects of charge were also completed, comparing results for the charged and neutral clusters with both the LC and SC potentials for the 20-atom cluster. For this cluster size, it was verified that the addition of a single electron significantly altered the thermodynamic behavior. With the $\mathrm{LC} \mathrm{Ga}_{20}^{+}$simulation most closely matching the overall nature of the experimental specific heat curve, the LC charged model was utilized for subsequent calculations.

In order to capture the intriguing one-atom differences in thermodynamic behavior, simulations were completed for two consecutive sizeranges. A series of very small, neutral clusters with sizes ranging from 7-12 atoms were modelled in an effort to simplify the system and test the size-limit for the definition of melting in small clusters. Larger cluster 
simulations, including $\mathrm{Ga}_{32}^{+}, \mathrm{Ga}_{33}^{+}, \mathrm{Ga}_{34}^{+}$and $\mathrm{Ga}_{35}^{+}$, allowed for direct comparison to experimental data.

In order to complete this set of FPMD simulations with the greatest accuracy and shortest simulation times, it was necessary to ensure that each cluster adequately explored configurational phase space. Although the microcanonical ensemble avoids the issue of thermostatting, the constraint of constant energy could serve to trap a given cluster in a nonground state configurational minimum, thus limiting the simulation from exploring the true ground state. This possible limitation was mitigated by the implementation of a parallel tempering wrapper code, as detailed in the following section.

\subsection{Parallel Tempering}

Clusters often exhibit complicated potential energy landscapes, with multiple sets of local minima separated by large potential energy barriers [142, 63]. In the microcanonical ensemble, where the energy to overcome the potential energy barriers is limited to the total energy of the simulation, the probability that a structure will become trapped in a local energy bin increases significantly [142]. Since gallium already exhibits an incredibly rich configurational landscape, as has been observed in both the clusters $[4,49,128]$ and bulk $[4,5]$, these FPMD simulations have a reasonable probability of becoming trapped in a local energy minimum. As previous research has demonstrated the importance of all configuration states being statistically accessible in order to ensure meaningful results and decrease total simulation time [142, 140], this research implements a parallel tempering wrapper code for VASP's microcanonical FPMD package.

This parallel tempering implementation considers each of the 19 constant energy simulations, as discussed in Section 4.3.2, to represent a different configurational trajectory. Every 100 time steps, all simulations are stopped and two configurations are randomly selected. The total and po- 
tential energies of the two configurations are used to compute an acceptance probability, which defines the rate at which two swaps are allowed, in accordance with Ref. [142]. In brief summary, the acceptance probability that configuration $\mathbf{r}_{i}$ will be swapped with configuration $\mathbf{r}_{j}$ in a microcanonical MD simulation is given by

$$
\operatorname{acc}\left(\mathbf{r}_{\mathrm{i}} \rightarrow \mathbf{r}_{\mathrm{j}}\right)=\min \left(1, \frac{\rho_{E, \mathbf{L}}\left(\mathbf{r}_{j}\right) T\left(\mathbf{r}_{\mathrm{j}} \rightarrow \mathbf{r}_{\mathrm{i}}\right)}{\rho_{E, \mathbf{L}}\left(\mathbf{r}_{i}\right) T\left(\mathbf{r}_{\mathrm{i}} \rightarrow \mathbf{r}_{\mathrm{j}}\right)}\right),
$$

where $T\left(\mathbf{r}_{j} \rightarrow \mathbf{r}_{i}\right)$ is a trial probability and $\rho_{E, \mathbf{L}}(\mathbf{r})$ is the equilibrium distribution or microcanonical weight:

$$
\rho_{E, \mathbf{L}}(\mathbf{r})=\zeta^{-1} \frac{1}{\sqrt{\operatorname{det} \mathbf{I}}} \Theta\left[E-U_{\mathbf{L}}(\mathbf{r})\right]\left[E-U_{\mathbf{L}}(\mathbf{r})\right]^{3 N / 2-4}
$$

with normalization factor $\zeta$, inertia matrix $\mathbf{I}$, and Heaviside step function $\Theta$. $U_{\mathbf{L}}(\mathbf{r})$ is the potential energy accounting for the energy contribution from the angular momentum, L. Eq. (4.3) ensures that the configurational "random walk" samples configuration space according to the equilibrium distribution from Eq. (4.4). In order to tune the acceptance rate in the canonical ensemble, $T\left(\mathbf{r}_{j} \rightarrow \mathbf{r}_{i}\right)$ is typically set to the Boltzmann distribution centered at $\mathbf{r}_{\mathbf{j}}$ with a width defined so that not too many or too few swaps are accepted. In microcanonical simulations, this quantity is replaced by the microcanonical weight from Eq. (4.4), yielding a greatly simplified

$$
\operatorname{acc}\left(\mathbf{r}_{\mathrm{i}} \rightarrow \mathbf{r}_{\mathrm{j}}\right)=\min \left(1,\left(\frac{\left[E_{i}-U_{\mathbf{L}}\left(\mathbf{r}_{j}\right)\right]\left[E_{j}-U_{\mathbf{L}}\left(\mathbf{r}_{i}\right)\right]}{\left[E_{i}-U_{\mathbf{L}}\left(\mathbf{r}_{i}\right)\right]\left[E_{j}-U_{\mathbf{L}}\left(\mathbf{r}_{j}\right)\right]}\right)^{3 N / 2-4}\right)
$$

This parallel tempering algorithm employs the Metropolis scheme [143], using a (pseudo-)random number generated between 0 and 1 to serve as an acceptance threshold, $\xi$. If $\operatorname{acc}\left(\mathbf{r}_{\mathrm{i}} \rightarrow \mathbf{r}_{\mathrm{j}}\right)>\xi$, the two configurations $\mathbf{r}_{i}$ and $\mathbf{r}_{j}$ are swapped. This random acceptance threshold accounts for the possibility that a less probable configuration will sometimes be thermodynamically sampled. 
Once the swap has either been made or rejected, the simulations are restarted and the process outlined above are re-run every 100 time steps. A general, schematic overview of the process is presented in Fig. 4.3, with each color. Due to computational considerations within VASP, some additional calculations are required before the restart can occur, as outlined in the following section.

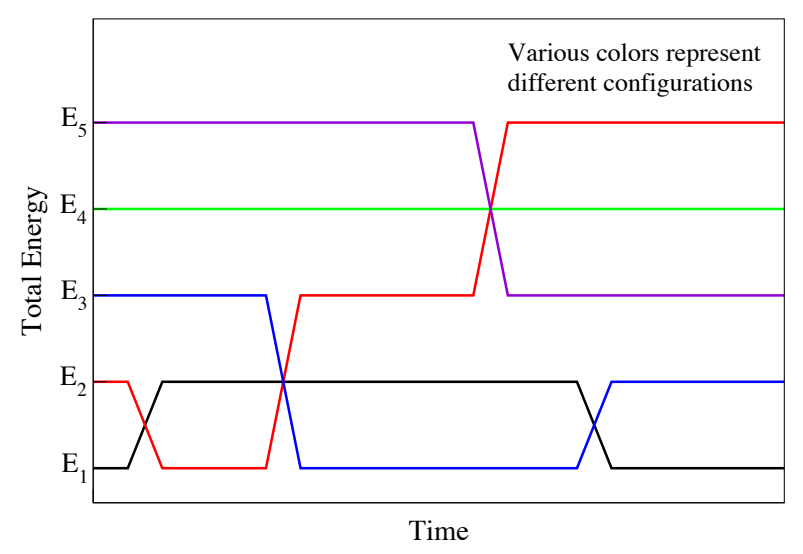

Figure 4.3. An illustrative example of the parallel tempering process in the microcanonical ensemble.

\subsubsection{VASP Swapping}

Given that the parallel tempering algorithm will make some configuration swaps, additional computational steps are required in order to ensure the calculations maintain constant energy within VASP. A constant energy restart calculation in VASP is based on a set of predictive position and velocity coordinates listed in the CONTCAR file. These coordinates are typically copied directly to the POSCAR file for the restart calculation and, coupled with the wavefunctions output to the WAVECAR file, give VASP the necessary information to begin a calculation in exactly the state that would have been calculated for the next time step, as if the calculation 
had never been stopped. When a configuration swap occurs, however, the velocities of the ions have to be scaled in order to account for the new kinetic energy required to maintain constant energy. Without considering the complications of the predictive coordinates within the CONTCAR file, a swap occurring between configuration $\mathbf{r}_{\mathbf{i}}$ and configuration $\mathbf{r}_{\mathbf{j}}$ would require velocities to be rescaled as

$$
\begin{aligned}
& S_{i}=\sqrt{\frac{E_{j}-U_{i}}{T_{i}}} \\
& S_{j}=\sqrt{\frac{E_{i}-U_{j}}{T_{j}}}
\end{aligned}
$$

where indices $i$ and $j$ characterize the configurations $\mathbf{r}_{\mathbf{i}}$ and $\mathbf{r}_{\mathbf{j}}, E$ is the total energy, $U$ is the potential energy, $T$ is the kinetic energy. $S_{i}$ and $S_{j}$ represent the scaling parameters for the velocities,

$$
\begin{aligned}
& v_{i_{\text {swap }}}=S_{i} v_{i} \\
& v_{j_{\text {swap }}}=S_{j} v_{j} .
\end{aligned}
$$

The scaled ion velocities following a swap are represented as $v_{i_{\text {swap }}}$ and $v_{j_{\text {swap }}}$, while $v_{i}$ and $v_{j}$ represent the original ion velocities before the swap. The complication occurs because the predictive velocities listed in the CONTCAR file are offset by $1 / 2$ time-step from the velocities that are used to calculate the kinetic energies. In other words, the predictive velocities are $v(t+\Delta t)$ while the kinetic energy is calculated according the half-step velocities, $v(t+1 / 2 \Delta t)$. On a normal restart, the VASP codes are written to use these values to take the next time step seamlessly. However, in order to swap configurations and velocities while maintaining constant energy, these settings cause energy inconsistencies and a work-around had to be determined.

Taking a single cycle to represent the 100 time-steps between parallel tempering swapping, this work-around involves repeating the final time- 
step calculation of the previous cycle as the first time step for the new cycle. In order to accomplish this, the velocities in the restart POSCAR must be modified to represent the velocities at time $t$ instead of the time $t+\Delta t$. This is done by working somewhat backwards through Eqs. (2.52)(2.54).

The first step involves reading the forces on each ion (as listed in the OUTCAR file) from the last iteration of the previous cycle, which define $F(t+\Delta t)$ from Eq. (2.54). Using the mass of a gallium ion, the acceleration $a(t+\Delta t)$ can be easily determined. The half-step velocities, $v(t+1 / 2 \Delta t)$, are listed in the predictive coordinates within the CONTCAR file, although it should be noted for technical purposes that these values are scaled according to the lattice parameters of the cell as well as the time step in order to render them non-dimensional. Using these velocities, the "old" velocities can be calculated according to

$$
\vec{v}(t)=S \cdot \vec{v}(t+\Delta t)-\frac{1}{2} \vec{a}(t+\Delta t) \Delta t
$$

where $S$ is the velocity rescaling parameter given in Eq. (4.6). In order for parallel tempering to work, all calculations must be linked for every time-step, so this algorithm must be employed for all parallel tempering subruns regardless of whether a swap occurs. This is accomplished by setting the scaling parameter to 1 for all non-swapped subruns, and equal to the appropriate swapping parameter from Eq. (4.6) if a swap does occur. It should be noted that a small error is introduced as the wavefunctions (listed in the WAVECAR file) correlate to the $t+\Delta t$ step as opposed to the $t$ step; however, through extensive testing it was determined that this error to the total energy amounted to $\sim 0.001 \%$. Aside from this small error, the algorithm yields two time-steps with identical values. To ensure this repetition did not skew the results, the final calculation for each cycle is excluded from all analysis. The following sections provide an overview of the set of analyses completed for each gallium cluster melting simulation. 


\subsection{Methods of Atomic Structure Analysis}

Ground state structural analysis has often been employed to explain the anomalous thermodynamic behavior observed in small clusters $[52,53,54$, $55,56,35,46]$. The global minimum structure is clearly important for identifying ground state properties of the system as well as an accurate latent heat description at finite temperatures. For gallium, however, the extensive polymorphism [4], indicating a complicated potential energy surface, has also been noted in the clusters $[49,50]$ and presents the additional challenge of clearly identifying a single ground state structure. One benefit of MD simulations is the production of statistical structural information in data generated over the course of a simulation containing between tens and hundreds of thousands of time iterations, which can help to mitigate the lack of statistics from the small number of atoms. These measures relate more directly to thermodynamic behavior, in that they quantify the structural response to increasing energy or temperature. Analyses such as root mean square bond length displacements $\left(\delta_{r m s}\right)$, mean square displacements (MSD), isomerization analysis [144] and averaged radial distribution functions, as discussed in the following sections, are well-suited to measuring the structural variation at finite temperature $[145,146]$.

\subsubsection{Radial distribution functions}

A radial distribution function (RDF) is simply a measure of the distribution of bond lengths throughout a structure. The radius separating all particles in a system are computed and binned (counted) according to length. For a bulk-like system with a large number of particles yielding ample radial statistics, the RDF is generally a smooth function of the radius. For these small systems, however, the RDF for an individual configuration has limited value as it is typically a set of disjointed peaks, since, as an example, the RDF for the 20-atom gallium structures account for only 190 total pair separations. 
Making use of the ample structural statistics available from molecular dynamics, a time-average RDF was found to be more instructive. These average RDF's (ARDF) were calculated for each constant energy subrun by summing the RDF at each time step, then dividing by the total number of time steps. This calculation gives the additional benefit of being able to analyze each structure at finite temperature, as opposed to the zerotemperature ground state, without having to choose an arbitrary structure from the simulations. Fig. 4.4 illustrates the difference for the gallium 20atom cationic structure, comparing the ARDF over 200 ps of simulation time to the single RDF for the zero-temperature optimized initial structure.

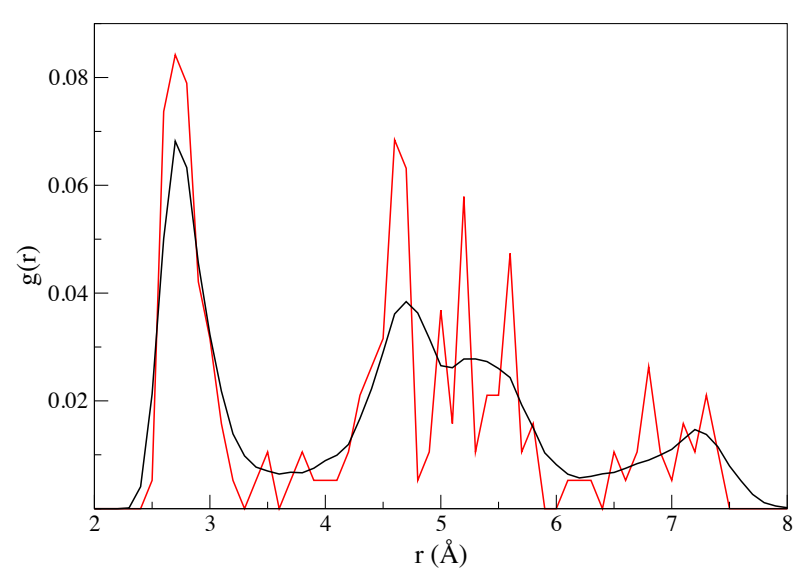

Figure 4.4. A comparison of the 20-atom cation ARDF over 200 ps (black) to the same simulation's zero-temperature, optimized initial structure (red).

\subsubsection{Root-mean square deviation}

Root-mean square (RMS) bond length analysis gives a measure of bond length variance as compared to the time-average bond lengths. For each simulation, the RMS bond length deviation, $\delta_{r m s}$, was computed at each constant energy according to 


$$
\delta_{r m s}=\frac{2}{N(N-1)} \sum_{i>j} \frac{\left(\left\langle r_{i j}^{2}\right\rangle_{t}-\left\langle r_{i j}\right\rangle_{t}^{2}\right)^{1 / 2}}{\left\langle r_{i j}\right\rangle_{t}},
$$

where $N$ is the total number of atoms, $r_{i j}$ represents the bond length between atoms $i$ and $j$ within the cluster at time $t$, and \langle\rangle$_{t}$ represents the time average of the quantity within the brackets. $\delta_{r m s}$ yields the total variance of bond displacement from the time-average bond length between two ions, $i$ and $j$.

In bulk matter, this unitless quantity (also known as the Lindemann index) serves as a clear indicator that a solid-liquid phase transition has occurred when the bond length variance exceeds 10-15\% [52] and has been shown to be a reasonable measure for molecular dynamics simulations [147]. However, the bond length variance in small cluster simulations is typically larger than that of bulk [33] and can render this quantity meaningless as a measure of melting transitions in clusters [53].

It should also be noted that $\delta_{r m s}$ becomes statistically invalid when a parallel tempering (configurational) swap is made between different energy or temperature trajectories. However, after extensive testing for our simulations, we have determined that this measure yields valid bond length variance trends at the lowest and highest temperatures. At the lowest temperatures, this is due to the low number of swaps that occur between structurally coherent, rigid structures. At the highest temperatures, the validity is due to swapping between molten, non-rigid structures where bond lengths vary throughout all trajectories.

Our testing revealed that in the transition region, where swapping might occur between molten and non-molten structures at a regular frequency, the actual values of $\delta_{r m s}$ become less valid. For this reason, the exact values of $\delta_{r m s}$ should be taken with caution within $\sim 100 \mathrm{~K}$ to each side of the melting temperature. For our purposes, however, we take RMS bond length variance as a supplemental analysis to the specific heat measures of melting. As a general measure of cluster rigidity at a given energy, we con- 
sider the $\delta_{r m s}$ analysis to be valid in the low and high temperature regimes, where it is clearly characteristic of solid-like and liquid-like behavior, respectively.

\subsubsection{Mean square displacement}

The mean square displacement (MSD) calculates the total structural displacement that occurs over a certain time interval. MSD is measured as a function of time, where $t$ represents the total time over which that displacement is measured. As opposed to choosing an average or arbitrary starting structure for the analysis, MSD samples a range of starting structures over the course of the simulation and then averages the structural displacements from each starting point. MSD is calculated per atom in the structure, but can be averaged over all atoms or subsets of atoms.

The MSD for atom $i$ at a given constant energy, $r_{i}^{2}$, can be calculated as

$$
\left\langle r_{i}^{2}(t)\right\rangle=\frac{1}{M} \sum_{m=1}^{M}\left[R_{i}\left(t_{0 m}+t\right)-R_{i}\left(t_{0 m}\right)\right]^{2},
$$

where $t_{0 M}$ represents a selected time origin, $t$ is the time over which the atomic displacement is measured, $R_{i}$ specifies the coordinates of the $i$ th atom at the specified time, and $M$ is the total number of time origins sampled.

As with $\delta_{r m s}$ discussed in the previous section, parallel tempering will affect the statistical validity of the MSD for each cluster. However, testing revealed that high and low temperature MSD were minimally affected by configuration swaps. Similar to $\delta_{r m s}$, the actual values for MSD should be taken with caution in the phase transition region $\sim 100 \mathrm{~K}$ to each side of the melting temperature.

While each of these methods of structural analysis illustrates a cluster's structural response to finite temperatures, one benefit of density functional theory calculations is the yield of electronic structure information 
that can be used to augment the understanding of bonding in these structures. Three methods of electronic structure analysis are covered in the following section.

\subsection{Methods of Electronic Structure Analysis}

For small systems governed partly or largely by quantum effects, the ability to analyze the electronic structure becomes a necessary component to truly understanding the system. One of the greatest advantages of density functional theory over classical modeling is the yield of (at least a good approximation to) the ground state electron density for any given structure. The following sections describe three different methods for analyzing electronic structure utilized in the course of this research.

\subsubsection{Bader analysis}

One of the simplest forms of electronic structure analysis lies in examining the distribution of charge. While VASP outputs a charge distribution map (CHGCAR), visual examination of this map typically gives limited information regarding the details of electronic structure. Bader analysis [148] is an analytic method for partitioning the charge distribution in a structure. The Bader analysis uses the electron density profiles, as given in the CHGCAR file, in order to draw charge density 'volumes' surrounding each atom. This analysis involves three steps: (i) identifying local maxima in the charge density; (ii) partitioning the space into Bader volumes, each containing one charge density maximum; and (iii) integrating the charge density within each volume and assigning this partial total charge to the nearest atom [149]. In this way, Bader analysis yields both the physical charge volume surfaces as well as the partial charges of each atom, where the charge contained within each volume is associated with the atom it surrounds. 


\subsubsection{Projected electronic density of states}

The spherical harmonic-projected electronic density of states (SH-PDOS) is a method for analyzing the angular momentum character of the valence electrons within a cluster. Based on the jellium model [150, 151], SH-PDOS assumes that the electronic structure of a cluster takes on spherical 'superatom'-like character, with its valence electrons filling molecular electron shells in a way similar to the aufbau principle for single atoms. This molecular shell structure goes as

$$
1 S^{2}, 1 P^{6}, 1 D^{10}, 2 S^{2}, 1 F^{14}, 2 P^{6}, 1 G^{18}, 2 D^{10}, 3 S^{2}, \ldots
$$

with the $S$-shell taking on a spherical angular momentum character, the $P$-shell taking on a lobed angular momentum character, etc., similar to the corresponding $s^{-}, p-$, etc. shells of an atom. This superatom molecular shell structure assumes electronic shell closings for molecules containing $2,8,18,20,34,40,58,68,70, \ldots$, valence electrons. Deemed "magic numbers," these shell closing correlate closely with the valence numbers for clusters observed to have the greatest stability in experimental measurements (abundance spectra, ionization potentials) [150].

For this research, a code developed by D. Schebarchov was used to analyze the angular momentum character of the valence electrons. Described in greater detail in Ref. [149], this code projects the Kohn-Sham (KS) orbitals $\psi(r, \theta, \phi)$, as defined in the WAVECAR file from VASP, onto spherical harmonic functions $Y_{l}^{m}(\theta, \phi)$. For a molecule with a center of mass, $R$, the angular momentum weight associated with each KS orbital, $\omega_{\ell}(R)$, is found by

$$
\omega_{\ell}(R)=\sum_{m=-\ell}^{\ell}\left|f_{\ell}^{m}(R)\right|^{2}
$$

where 


$$
f_{\ell}^{m}(R)=\int_{\Omega} \int_{0}^{R} Y_{\ell}^{m *}(\theta, \phi) \psi(r, \theta, \phi) r^{2} d r d \theta d \Omega,
$$

with $\Omega$ as the solid angle and $d \Omega=\sin \theta d \theta d \phi$. In order to make the visualization easier, each of the weights was broadened around the corresponding KS eigenvalue using Gaussian smearing of 0.1 . This analysis defines the angular momentum character of each KS orbital, which can then be plotted according to the orbital energies (eigenvalues), giving a pictorial representation of the electronic DOS comprised of angular momentum components.

\subsubsection{Electron localization function}

The electron localization function (ELF) $[152,153,154]$ is a method designed to measure an electron pair probability, or the likelihood of finding two electrons of the same spin in neighboring regions of space. This can best be described by example, with respect to reference electron $\mathbf{1}$ and a second electron 2: if the probability of finding $\mathbf{2}$ in the vicinity of $\mathbf{1}$ is very low, then one can say that $\mathbf{1}$ is very localized, whereas if the likelihood that $\mathbf{2}$ will be found near $\mathbf{1}$ is high, $\mathbf{1}$ is not so localized. By this definition, electron localization is only a relative concept and has no upper bound, which is not so useful for analysis. In order to better quantify ELF, the measure was recalibrated to the localization of a free electron gas on a scale of 0 to 1. The upper end, 1 , relates to perfect localization, or zero probability of finding $\mathbf{2}$ in the vicinity of $\mathbf{1}$. A value of $E L F=0.5$ correlates to an electron gas-like probability distribution for electron pairs.

Overall, ELF depends on the total electronic density, its gradient and the electron kinetic energy density [152]. ELF analysis is a standard tool available within VASP, the results of which can be easily visualized. Regions of electron localization with the same probability are drawn threedimensionally as isosurfaces based on ELF values, which can serve as a useful tool in classifying chemical bonding $[153,154]$. In this research, it 
was found to be particularly useful in distinguishing covalent bonding, or lack thereof, within the clusters, where ELF-identified covalent bonding manifests as a region of relatively strong electron localization (generally taken to minimally be ELF $>0.6$ ) that lies generally centered between two nuclei along the bond axis.

With electronic structure analysis yielding more information on the nature of bonding in these small gallium clusters, a more complete picture of their interesting thermodynamic properties can be developed. In order to describe their thermodynamic behavior, however, methods of thermodynamic analysis must be employed. Two such methods are outlined here.

\subsection{Methods of Thermodynamic Analysis}

Two methods for evaluating the specific heat curves were utilized over the course of the research. The initial method calculated a single, discrete specific heat value for every simulation energy. Later, the more sophisticated multiple histogram method was employed, which makes use of the ample statistical information from each MD run in order to calculate specific heat values continuously across a range of energies/temperatures. Each method is outlined in more depth in the following sections.

\subsubsection{Discrete Analysis}

Initial calculations of thermodynamic quantities employed the analysis outlined in Ref. [155], which uses kinetic energy variance as a measure of heat capacity and temperature in the microcanonical ensemble. From this reference, the microcanonical specific heat is defined as

$$
\begin{aligned}
c_{V}\left(E_{i}\right)= & \left(\frac{3 N-6}{2}-1\right) \\
& {\left[N\left(\frac{3 N-6}{2}-1\right)-N\left(\frac{3 N-6}{2}-1\right) \frac{Y_{i}}{Z_{i}^{2}}\right]^{-1}, }
\end{aligned}
$$


where $N$ is the number of atoms, $i$ is the index that runs over each of the 19 constant energy subruns for the simulations, and $Y$ is defined as

$$
Y_{i}=\left\langle K_{i}\right\rangle_{t}^{2}\left\langle K_{i}^{-2}\right\rangle_{t}
$$

with kinetic energy, $K_{i}$. $Z$ is given as

$$
Z_{i}=\left\langle K_{i}\right\rangle_{t}\left\langle K_{i}^{-1}\right\rangle_{t}
$$

The microcanonical temperature can be defined as

$$
T_{d}\left(E_{i}\right)=\frac{1}{k_{B}}\left[\left(\frac{3 N-6}{2}-1\right)\left\langle K_{i}^{-1}\right\rangle_{t}\right]^{-1} .
$$

This method produces discretely sampled data, i.e., one specific heat value per simulation energy, which proved to be a significant limitation for our first-principles MD study. With our simulations sampling only 19 energies across a broad energy range, this discrete analysis yields rough, sparse specific heat curves with large error bars for the melting temperatures and lacking sufficient detail to discern the overall nature of the melting transition. Without a priori knowledge of the melting temperature or the use of computationally impractical numbers of subruns for first-principles MD, discrete measures of thermodynamic quantities were of limited use. As an additional limitation, the discrete analysis yields the specific heat only as a function of energy, which can then be related back to a dynamical temperature through a relationship to the time-average kinetic energy or by utilizing the average temperatures of each subrun. In this way, it allows only indirect comparisons to the experimentally measured specific heat curves which use temperature as the independent variable.

\subsubsection{Multiple Histogram Analysis}

To fully exploit the ample statistical data generated in each simulation, we have used the multiple-histogram (MH) method $[156,157,158]$ to charac- 
terize thermodynamic quantities continuously across the simulation energy range. This method uses the distribution of potential energies over the course of a simulation to extract the configurational and total density of states, $\Omega_{C}(U)$ and $\Omega(E)$ respectively, from which the configurational and total entropies can be derived. These two quantities allow us to calculate both microcanonical and canonical thermodynamic quantities, including the specific heats and temperatures.

Generally, our derivation of the microcanonical MH equations follows the procedure outlined by Calvo and Labastie [159]. We calculate the normalized probability distribution for potential energies, $U$, at total energies, $\mathrm{E}$, given by

$$
p^{\text {theor }}(U ; E)=\frac{\Omega_{K}(E-U) \Omega_{C}(U)}{\Omega(E)},
$$

with kinetic energy density of states, $\Omega_{K}$, and configurational density of states, $\Omega_{C}$. The kinetic energy density of states evaluates analytically to:

$$
\Omega_{K}(K)=B K^{\frac{\nu}{2}-1},
$$

where $B$ is a constant and $\nu$ is the number of degrees of freedom, which will be $3 N-6$ if the impulse and angular momentum are zero.

The configurational density of states can only be determined numerically. For a simulation carried out at constant total energy, $E_{i}$, the probability of finding a configurational energy, $U_{j}$, in the range $\left[U_{j}-\Delta U / 2, U_{j}+\right.$ $\Delta U / 2$ ] is given by $p\left(U_{j}, E_{i}\right) \Delta U$ (or $p_{i j}$ for simplicity), numerically defined by

$$
p_{i j}^{s i m}=\frac{n_{i j}}{\sum_{j} n_{i j}} .
$$

The quantity $n_{i j}=n\left(U_{j} ; E_{i}\right)$ is determined by counting the number of configurational energies that fall into the $\left[U_{j}-\Delta U / 2, U_{j}+\Delta U / 2\right]$ bin over the course of a simulation at $E_{i}$. For each constant energy subrun, $n_{i j}$ will 
form a histogram that is Gaussian in nature. By recasting the theoretical probability, given in Eq. (4.17), in terms of $E_{i}$ and $U_{j}$, and setting this equal to our simulated probability from Eq. (4.19), we obtain the expression

$$
\frac{\Omega_{K}\left(E_{i}-U_{j}\right) \Omega_{C}\left(U_{j}\right)}{\Omega\left(E_{i}\right)}=\frac{n_{i j}}{\sum_{j} n_{i j}} .
$$

Now recognizing that the kinetic energy density of states is a known quantity, the total unnormalized counts obtained by a microcanonical numerical simulation simplifies to

$$
n_{i j}=\frac{B K^{\frac{\nu}{2}-1} \Omega_{C}\left(U_{j}\right)}{\Omega\left(E_{i}\right)} \sum_{j} n_{i j} .
$$

Rewriting the kinetic energy in terms of the potential and total energy and writing out the degrees of freedom, this becomes

$$
n_{i j}=\frac{B\left(E_{i}-U_{j}\right)^{\frac{3 N-8}{2}} \Omega_{C}\left(U_{j}\right)}{\Omega\left(E_{i}\right)} \sum_{j} n_{i j} .
$$

Finally, since $\sum_{j} n_{i j}$ and $\Omega\left(E_{i}\right)$ are both constant for a given constant energy simulation, these can be combined yielding a simplified

$$
n_{i j}=\alpha_{i}\left(E_{i}-U_{j}\right)^{\frac{3 N-8}{2}} \Omega_{C}\left(U_{j}\right)
$$

where $\alpha_{i}$ is a constant

$$
\alpha_{i}=B \sum_{j} n_{i j} / \Omega\left(E_{i}\right)
$$

In order to work with the configurational entropy, defined as $S_{j}=$ $k_{B} \ln \Omega_{j}$, where $\Omega_{j}$ is the binned configurational density of states, $\Omega_{C}\left(U_{j}\right)$, the $\log$ of equation 4.23 is taken,

$$
\frac{S_{j}}{k_{B}}+\frac{(3 N-8)}{2} \ln \left(E_{i}-V_{j}\right)+A_{i}-\ln n_{i j}=0
$$


with $A_{i}=\ln \left(\alpha_{i}\right)$, which defines the additive constant that relates each individual constant energy histogram $\left(n_{i j}\right)$ to its neighbor $\left(n_{i+1, j}\right)$, thus "stitching together" the configurational entropies for the individual $E_{i}$ subruns. Eq. (4.25) assumes that the theoretical and simulated probability densities are exactly equal. In reality, there will be an absolute uncertainty in their difference [146],

$$
\ln p_{i j}^{\text {theor }}-\ln p_{i j}^{\text {sim }}=\frac{1}{\sqrt{n_{i j}}}
$$

Accounting for this difference, Eq. (4.25) becomes

$$
\frac{S_{j}}{k_{B}}+\frac{(3 N-8)}{2} \ln \left(E_{i}-V_{j}\right)+A_{i}-\ln n_{i j}=\frac{1}{\sqrt{n_{i j}}},
$$

where $A_{i}$ and $S_{j}$ are both unknown. The configurational entropy will only be valid within the interval defined by the width of the potential energy distribution for a certain $E_{i}$ subrun, but $\Omega_{j}$ remains valid across the energy range, independent of our choice of $E_{i}$. In this way, the overlapping of the histograms (as illustrated in Fig. 4.5) allows us to relate the $A_{i}$ 's between adjacent potential energy distributions.

In general, we want to minimize the difference between the simulated and theoretical simulations for all $i$ and $j$. A simple approach to this minimization is to complete a linear least squares regression for Eq. 4.27, thereby minimizing the sum of the squares of the differences between the simulated and theoretical probabilities given as

$$
\sum_{i j} n_{i j}\left(\frac{S_{j}}{k_{B}}+\frac{(3 N-8)}{2} \ln \left(E_{i}-V_{j}\right)+A_{i}-\ln n_{i j}\right)^{2}=1 .
$$

By differentiating with respect to the two unknowns, $S_{j}$ and $A_{i}$, we can solve for each quantity individually. More mathematical detail for deriving the analytic expressions for $S_{j}$ and $A_{i}$ are provided in Appendix A. Once the configurational entropy and additive constants are defined, the 
microcanonical and canonical MH specific heats can be easily determined, as outlined in greater detail in the following section.

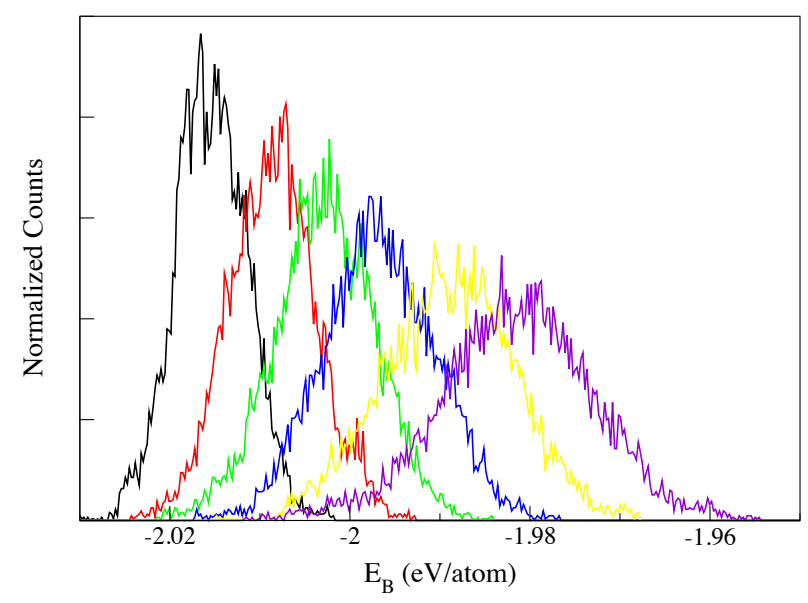

Figure 4.5. An illustrative example of the overlapping histograms, shown here for the lowest 6 energies of a 20-atom gallium cluster simulation.

\section{MH Specific Heats}

Given a continuous configurational and total density of states, the equations for MH specific heat are defined by Ref. [160]. Microcanonical thermodynamic quantities can be obtained by directly taking derivatives of the entropy. The microcanonical temperature is determined as

$$
T\left(E_{j}\right)=\left[\frac{\partial S_{j}}{\partial E_{j}}\right]^{-1}
$$

where the $E_{j}$ 's now represent the relevant total energies spanning the range of the simulation, as opposed to the 19 discrete constant energies for each microcanonical subrun defined by $E_{i}$. The microcanonical specific heat can be found in taking the derivative again, 


$$
C_{V}\left(E_{j}\right)=\left[\frac{\partial T\left(E_{j}\right)}{\partial E_{j}}\right]^{-1} .
$$

The canonical specific heat is typically defined as

$$
C_{V}(T)=\left[\frac{\partial \mathscr{E}}{\partial T}\right]
$$

where $\mathscr{E}$ defines the average value of the total energy, defined as the sum of the kinetic and potential energies as

$$
\mathscr{E}=\langle U+K\rangle_{T o t} .
$$

The specific heat can then be obtained by taking the derivative of this sum with respect to temperature. The kinetic energy average is obtained from the equipartition function, as

$$
\langle K\rangle_{T o t}=\frac{1}{2}(3 N-3) k_{B} T,
$$

which accounts for the rotational and vibrational kinetic energy of a system, with no translation for the center of mass.

The expression for the average potential energy is simply the expectation value

$$
\langle U\rangle_{T o t}=\frac{\int W \Omega_{C}(W) \exp \left(\frac{-W}{k_{B} T}\right) d W}{\int \Omega_{C}(W) \exp \left(\frac{-W}{k_{B} T}\right) d W},
$$

with $W$ being a dummy variable for the integration representing the potential energies of the simulation. It should be noted that, as written, this quantity will evaluate to $\infty / \infty$. In order for this quantity to be finite, there must be a minimum value of $W_{0}$, where the integrals can be redefined in terms of these limits as

$$
\langle U\rangle_{T o t}=\frac{\int_{W_{0}}^{\infty} W \Omega_{C}(W) \exp \left(\frac{-W}{k_{B} T}\right) d W}{\int_{W_{0}}^{\infty} \Omega_{C}(W) \exp \left(\frac{-W}{k_{B} T}\right) d W} .
$$


The integral can then be shifted according to $W^{\prime}=W-W_{0}$, which places the exponent only over positive quantities of $W^{\prime}$ and avoids the undefined infinite fraction. This shift also implies $d W^{\prime}=d W$, yielding the integrals

$$
\langle U\rangle_{T o t}=\frac{\int_{0}^{\infty}\left(W^{\prime}+W_{0}\right) \Omega_{C}\left(W^{\prime}+W_{0}\right) \exp \left(\frac{-\left(W^{\prime}+W_{0}\right)}{k_{B} T}\right) d W^{\prime}}{\int_{0}^{\infty} \Omega_{C}\left(W^{\prime}+W_{0}\right) \exp \left(\frac{-\left(W^{\prime}+W_{0}\right)}{k_{B} T}\right) d W^{\prime}}
$$

Since $W_{0}$ is a constant, this expression can be rewritten as

$$
\begin{aligned}
\langle U\rangle_{T o t} & =\frac{\exp \left(\frac{-W_{0}}{k_{B} T}\right) \int_{0}^{\infty}\left(W^{\prime}+W_{0}\right) \Omega_{C}\left(W^{\prime}+W_{0}\right) \exp \left(\frac{-\left(W^{\prime}\right)}{k_{B} T}\right) d W^{\prime}}{\exp \left(\frac{-W_{0}}{k_{B} T}\right) \int_{0}^{\infty} \Omega_{C}\left(W^{\prime}+W_{0}\right) \exp \left(\frac{-\left(W^{\prime}\right)}{k_{B} T}\right) d W^{\prime}} \\
& =\frac{\int_{0}^{\infty}\left(W^{\prime}+W_{0}\right) \Omega_{C}\left(W^{\prime}+W_{0}\right) \exp \left(\frac{-\left(W^{\prime}\right)}{k_{B} T}\right) d W^{\prime}}{\int_{0}^{\infty} \Omega_{C}\left(W^{\prime}+W_{0}\right) \exp \left(\frac{-\left(W^{\prime}\right)}{k_{B} T}\right) d W^{\prime}}
\end{aligned} .
$$

The final step is multiplying out the $\left(W^{\prime}+W\right)$ factor in the numerator and splitting the fraction, such that the equations simplifies to

$$
\begin{aligned}
\langle U\rangle_{T o t}= & \frac{\int_{0}^{\infty} W^{\prime} \Omega_{C}\left(W^{\prime}+W_{0}\right) \exp \left(\frac{-\left(W^{\prime}\right)}{k_{B} T}\right) d W^{\prime}}{\int_{0}^{\infty} \Omega_{C}\left(W^{\prime}+W_{0}\right) \exp \left(\frac{-\left(W^{\prime}\right)}{k_{B} T}\right) d W^{\prime}} \\
& +\frac{W_{0} \int_{0}^{\infty} \Omega_{C}\left(W^{\prime}+W_{0}\right) \exp \left(\frac{-\left(W^{\prime}\right)}{k_{B} T}\right) d W^{\prime}}{\int_{0}^{\infty} \Omega_{C}\left(W^{\prime}+W_{0}\right) \exp \left(\frac{-\left(W^{\prime}\right)}{k_{B} T}\right) d W^{\prime}} . \\
= & \frac{\int_{0}^{\infty} W^{\prime} \Omega_{C}\left(W^{\prime}+W_{0}\right) \exp \left(\frac{-\left(W^{\prime}\right)}{k_{B} T}\right) d W^{\prime}}{\int_{0}^{\infty} \Omega_{C}\left(W^{\prime}+W_{0}\right) \exp \left(\frac{-\left(W^{\prime}\right)}{k_{B} T}\right) d W^{\prime}}+W_{0}
\end{aligned}
$$

This expression must be evaluated numerically, such that $T=T_{j}$ and $U=$ $U_{j} . K_{j}$ can also be determined numerically in taking $T=T_{j}$ in Eq. (4.33), with the average total energy numerically defined as 


$$
\mathscr{E}_{j}=K_{j}+U_{j}
$$

With this definition, the numerical derivative of the average total energy, $\mathscr{E}_{j}$, can be easily calculated to obtain the canonical specific heat, $C_{V}\left(T_{j}\right)$,

$$
C_{V}\left(T_{j}\right)=\frac{\mathscr{E}_{j+1}-\mathscr{E}_{j}}{T_{j+1}-T_{j}}
$$

Eqs. 4.30 and 4.40 yield continuous microcanonical and canonical specific heat curves from only 19 constant energy simulations. Examples of these continuous specific heat curves in comparison to the results of the discrete specific heat analysis are illustrated in Figure 4.6. 


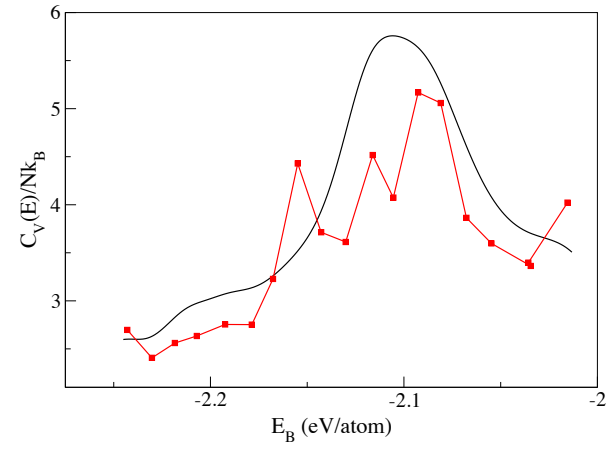

(a) Microcanonical SC $\mathrm{Ga}_{20}$

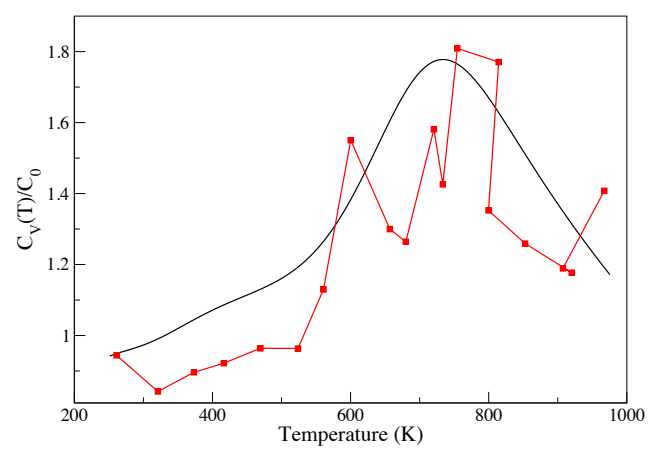

(c) Canonical SC Ga $\mathrm{Ga}_{20}$

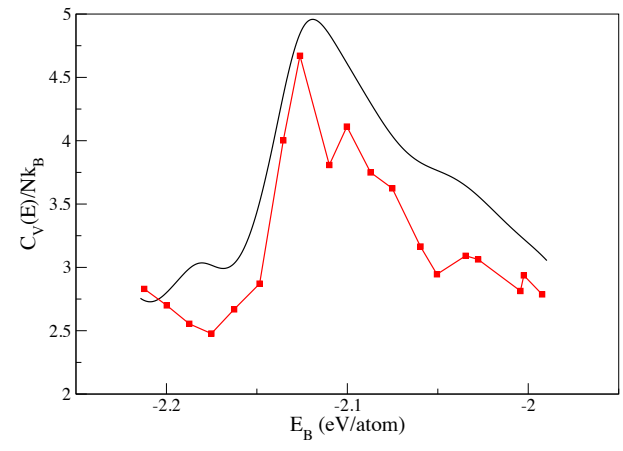

(b) Microcanonical LC Ga $\mathrm{La}_{20}^{+}$

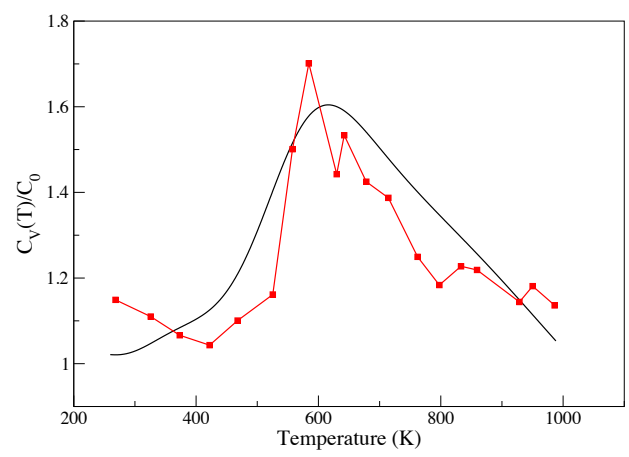

(d) Canonical LC Ga $\mathrm{La}_{20}^{+}$

Figure 4.6. Comparisons of the microcanonical and canonical specific heat curves using the discrete (red with square) vs. MH (black) methods of analysis for $\mathrm{SC} \mathrm{Ga}_{20}$ and $\mathrm{LC} \mathrm{Ga}_{20}^{+}$clusters, illustrating the benefits of the $\mathrm{MH}$ analysis. 
In general, the $\mathrm{MH}$ method works by quantifying the region of configurational instability that exists in the solid-liquid phase coexistence. Illustrated in Fig. 4.7, the peak in the microcanonical specific heat curve is directly correlated to the pronounced dip in the total unnormalized configurational energy counts, $n_{i j}$. This dip in the configurational counts represents the configurational entropy apex of the melting energy, with the entropy of the solid and liquid phases to each side being lower. The peak in the microcanonical specific heat curve arises from the higher configurational entropy that exists in this region of instability.

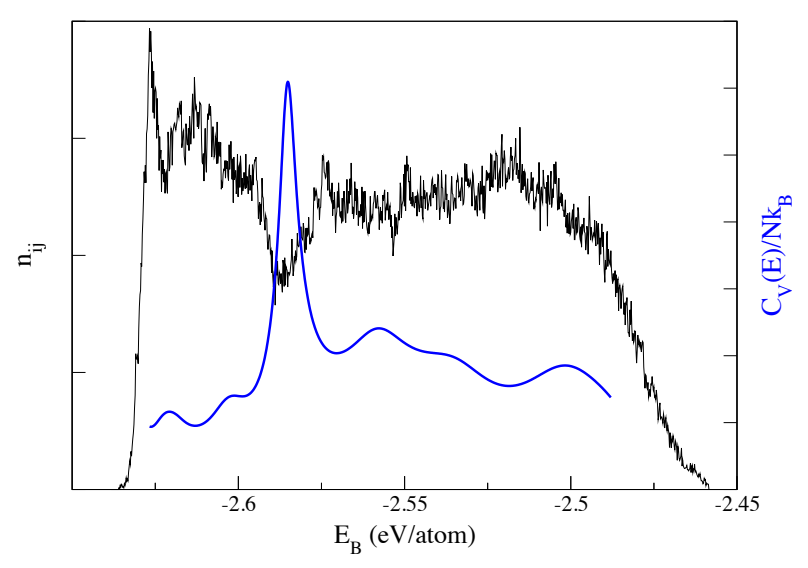

Figure 4.7. The total unnormalized configurational energy counts (black) is compared to the microcanonical specific heat curve (blue) for $\mathrm{Ga}_{35}^{+}$, illustrating the configurational region of instability surrounding the melting energy.

Armed with this set of analysis tools, an initial set of FPMD simulations were completed for the 20-atom gallium cluster in order to test the effects of charge and pseudopotential on the melting simulations. The following chapter gives the details of these simulations and results. 


\section{Chapter 5}

\section{0-atom Gallium}

All initial simulations were completed on the 20-atom cluster, which served as a test cluster for determining the charge state and pseudopotential model best suited to capture the thermodynamic behavior of these small systems. As published by this author and colleagues in Ref. [161], the following sections describe the results of these initial tests, to include descriptions of the atomic and electronic structural analysis completed for each simulation.

\subsection{Initial settings}

In order to understand the settings needed to accurately capture the odd melting behavior in these small systems, both a neutral and cationic $\mathrm{Ga}_{20}$ simulation were run using two pseudopotentials: a large-core (LC) model, treating the d-electrons as non-binding with a $4 s^{2} 4 p^{1}$ valence set, and the small-core (SC) model, treating the d-electrons as binding with a $4 s^{2} 3 d^{10} 4 p^{1}$ valence set. Initial cluster configurations were derived from two general structural classes: (A) a stacked-plane structure, similar to the global minimum $\mathrm{Ga}_{20}$ and $A l_{20}$ structures identified during extensive genetic algorithm searches [48, 162, 50, 129]; (B) a capped-sphere structure discovered in the course of our initial classical MD testing, as described in greater depth in Section 4.2. The stacked-plane structure resembles a series of 
planes consisting of alternating hexagons or pentagons, each separated by a single atom. The capped-sphere configuration features a trapped-atom surrounded by a spherical cage-like atomic structure, with an asymmetric tetragonal cap protruding from the sphere. The two structural classes, as well as the cohesive energies, are illustrated in Fig. 5.1. The cohesive energies are calculated according to

$$
E_{\mathrm{coh}}=E(G a)-E\left(G a_{n}\right) / n
$$

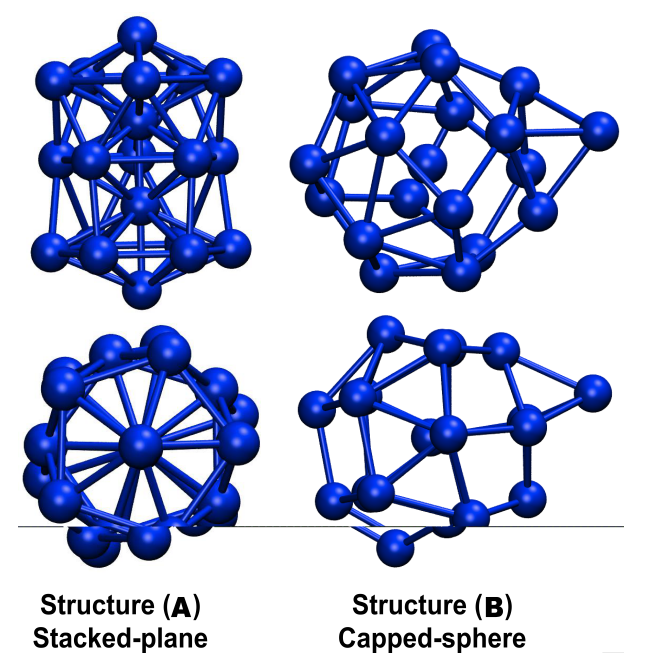

Figure 5.1. The two structural classes used to initialize each simulation. The top and bottom rows represent two views of (left) structural class (A), the stacked-plane structure $\left(E_{c o h}=2.334 \mathrm{eV} /\right.$ atom) and (right) structural class $(\mathbf{B})$, the capped-sphere structure $\left(E_{c o h}=2.354 \mathrm{eV} /\right.$ atom $)$. Here, $E_{c o h}$ was calculated via the small-core PAW potential for neutral charge state Atomic coordinates are given in Tab. B.6 and B.7 for the stacked-plane and capped-sphere structure, respectively.

As previously mentioned in Section 4.3.2, density-functional theory (DFT) conjugate-gradient optimizations were completed for each initial 
structure using Projector-Augmented Wave (PAW) potentials [130, 131], with an energy cut-off of $350 \mathrm{eV}$ and the GGA-PW91 exchange-correlation functional [127, 132], as implemented in VASP 5.2 [113, 114, 115, 116]. Such optimizations were performed for the $\mathrm{Ga}_{20}$ and $\mathrm{Ga}_{20}^{+}$clusters with both the large-core (LC: core d-electrons, $4 s^{2} 4 p^{1}$ valence set) and the small-core (SC: valence $d$-electrons, $4 s^{2} 3 d^{10} 4 p^{1}$ valence set) PAW potentials. The aim of the initial optimization was to obtain a reasonable starting geometry for the simulation, rather than an attempt to rigorously identify the ground state structure.

Molecular dynamics (MD) simulations were performed using the BornOppenheimer MD implementation in VASP. All settings were maintained from the geometry optimization calculations aside from the energy cut-off which was reset to the default values: $282.7 \mathrm{eV}$ for the SC PAW; $134.7 \mathrm{eV}$ for the LC PAW. Each simulation included 10 ps of canonical equilibration for 37 temperatures spanning 200-2000 K, enabling the selection of 19 runs that adequately spanned the temperature range of experimental interest with approximately evenly-spaced average energy values. For these 19 simulations, the canonical equilibration was followed by a minimum of 92 ps microcanonical relaxation and simulation time per energy, per cluster. Using a time step of 2 fs for all energies, the total energy oscillations for each microcanonical simulation were $\sim 0.001 \mathrm{eV}$, with no noticeable drift. For both optimizations and MD, we added corrections for the full dipole moment in all directions in order to treat the cationic structure, as outlined in Sec. 4.3.2.

\subsection{Atomic structure analysis}

The initial structures for the neutral $\mathrm{LC} \mathrm{Ga}_{20}, \mathrm{SC} \mathrm{Ga}_{20}$, and charged SC $\mathrm{Ga}_{20}^{+}$simulations were each derived from structural class (A). The initial configurations for the two neutral clusters are nearly identical, each featuring the hexagonal-pentagonal-pentagonal stacked-planes separated 
and capped by single atoms, with almost indiscernible bond-length differences arising from their differing PAW potentials. These structures are very similar to the global minimum $A l_{20}$ structure determined by previous work [129]. The initial structure for the SC gallium cation varies slightly in that one of the capping atoms is absorbed into one of the planes, giving it a hexagonal-pentagonal-hexagonal stacked-plane configuration, more resembling the global minimum $\mathrm{Ga}_{20}$ structure determined by Drebov et al. [50].

The initial configuration for the $\mathrm{LC} \mathrm{Ga}_{20}^{+}$simulation was derived from structural class (B). This structure was the lowest energy configuration we had discovered for this charge/PAW setting at the onset of the simulations and is quite distinct from the other initial structures. It features a central atom enclosed in a nearly-spherical structure, with an asymmetric tetragonal cap protruding from the sphere. The central atom is quite isolated from the enclosing atoms, with center-sphere bond lengths $\sim 20 \%$ longer than sphere-sphere bonds.

A normalized average radial distribution function (ARDF) was computed for each simulation at each sub-run energy in order to analyze the effect of temperature on structural variation, as illustrated in Fig. 5.2. These ARDF's were calculated per subrun energy by summing the RDF at each time step, then dividing by the total number of time steps. Since the different PAW potentials generate unique energy ranges spanned by each simulation, the average temperatures of each sub-run were used in order to compare and contrast ARDF's between simulations. The $\mathrm{LC} \mathrm{Ga}_{20}$ and SC $\mathrm{Ga}_{20}^{+}$clusters have the same basic structure (stacked plane, type (A)), despite both the different pseudopotential and charge state. At low average temperatures (top two panels of Fig. 5.2), each of the stackedplane clusters exhibit split second nearest-neighbor peaks at $4.6 \AA$ and 5.2 $\AA$ which disappear at higher energies where the clusters are in a liquidlike state. In contrast, the $\mathrm{LC} \mathrm{Ga}_{20}^{+}$structure displays a shoulder on the first peak and no splitting of the second nearest-neighbor peak. All clus- 
ters converge to the same liquid phase averaged structure at high average temperatures, as illustrated by the bottom panel of Fig. 5.2.

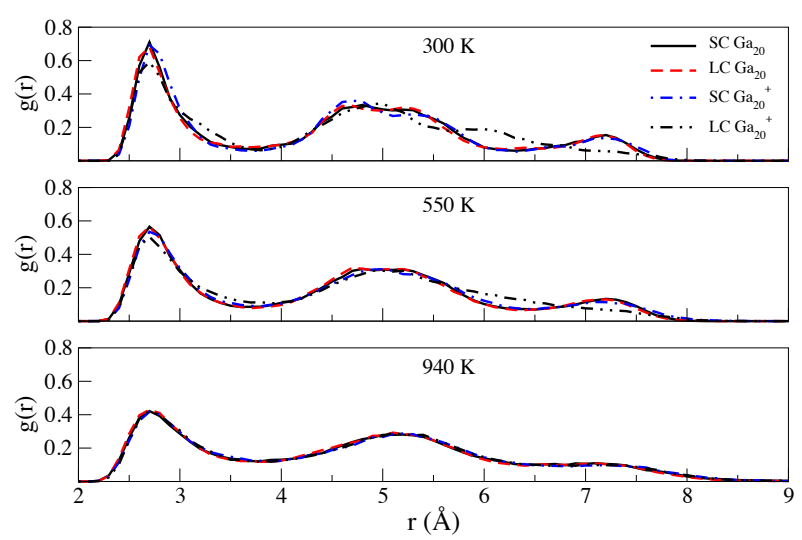

Figure 5.2. Normalized ARDF's comparing 3 constant energy sub-runs with comparable average temperatures. The SC $\mathrm{Ga}_{20}$ (black solid), LC $\mathrm{Ga}_{20}$ (red dash) and SC $\mathrm{Ga}_{20}^{+}$(blue dash-dot) simulations, each initialized with structural class $(\mathbf{A})$, in contrast with the $\mathrm{LC} \mathrm{Ga}_{20}^{+}$(black dash-dotdot), derived from structural class (B), illustrating the marked differences in atomic structures at low energies/average temperatures.

\subsection{Effect of parallel tempering}

To investigate the efficacy of parallel tempering (PT) in exploring configurational phase space, we analyzed the minimum energy structure explored during the course of each simulation and compared it to the optimized initial configuration. The results are summarized in Fig. 5.3, which shows the initial configuration for each simulation as well as the minimum energy structure discovered during parallel tempering, which have been optimized to their zero-temperature ground state for purposes of comparison. For the SC neutral, LC neutral and SC ion, the PT-derived structures (designated as PT) have lower cohesive energy than their corresponding 
starting structures. While each of these PT structures represents a modification to the stacked-plane configuration, which has been reported as the global minimum structure in previous research $[48,50,51]$, the sampling of the low energy isomers serves to illustrate that parallel tempering can reduce the importance of initializing the MD simulations with the global minimum structure.

For the SC and LC neutral simulations, the minimum energy structure for 20-atom gallium determined by a multi-stage genetic algorithm search [50] is also included for reference. The PT-derived structures have $\sim 0.1$ $\mathrm{eV}(0.005 \mathrm{eV} /$ atom) lower cohesive energy than the reference structure, which is not surprising due to the differing underlying levels of theory: the plotted cohesive energy was calculated using the relevant PAW potential (either SC or LC gallium PAW) with the GGA-PW91 functional, while Drebov et al. [50] use the PBE and TPSS functionals with TZVP basis sets.

The influence of the parallel tempering scheme on the simulations can be appreciated in the significant structural transitions observed during the course of the SC $\mathrm{Ga}_{20}^{+}, \mathrm{LC} \mathrm{Ga}_{20}^{+}$and $\mathrm{SC} \mathrm{Ga}_{20}$ simulations. Each of these structures are illustrated by the PT2 structures in Figure 5.3, which represent the lowest energy structures observed for the duration of time the structure remained in swapped configurations, optimized to a ground state energy for purposes of comparison with the other structures. The longest duration transition occurred during the SC cationic simulation, which was initialized with a stacked-plane configuration of structural class (A) and, through ARDF analysis, remains in this configuration for the majority of simulation time at low-energies (below melting). During the course of the microcanonical simulation, an amorphous structure undergoes a parallel tempering swap to a lower-energy sub-run where it quickly relaxes into the capped-sphere structure of structural class (B). ARDF analysis and visual inspection reveal that the capped-sphere structure persists for the next 27 ps, at which time it is again swapped to a higher energy sub-run. Fig. 5.4 illustrates the ARDF signature for the capped-sphere 
structure over the course of 20 ps spanning between parallel tempering swaps, in stark contrast to the stacked-plane signatures from neighboring energies over the same time frame. This figure also includes the ARDF analysis for a comparable average temperature sub-run of the $\mathrm{LC} \mathrm{Ga}_{20}^{+}$ simulation, which was initialized with and remains in the capped-sphere configuration.

The stacked-plane to capped-sphere structural transition observed for the SC $\mathrm{Ga}_{20}$ remained in the capped-sphere configuration for 10 ps between swaps. The transition for the $\mathrm{LC} \mathrm{Ga}_{20}^{+}$simulation reversed these transformations, going from the initial capped-sphere configuration to a higher-energy version of the stacked-plane isomer, and persisted for only 3 ps between swaps. Although this structure optimizes to a lower energy, it does not represent the lowest energy configuration explored during the course of the simulation and, given the short duration of its persistence, does not appear to be the most stable configuration at finite temperature for these charge/PAW settings. This illustrates that the lowest energy structure may not always be the most relevant for melting, as demonstrated by the convergence of the specific heat curves (as discussed in Section 5.4). The LC neutral was the only simulation for which a structural transition was not observed. Here, the capped-sphere structure was optimized using the LC pseudopotential for purposes of comparison. While each of these swaps was of shorter duration, they serve to clearly demonstrate the competing nature of these two distinct isomers at finite temperature.

This example illustrates a very important point: a fully converged simulation should demonstrate the same melting behavior, regardless of starting structure. Convergence time is therefore related to a reasonable choice of a starting structure that is physically relevant at low temperature. We discuss our measures of convergence in the following section. 

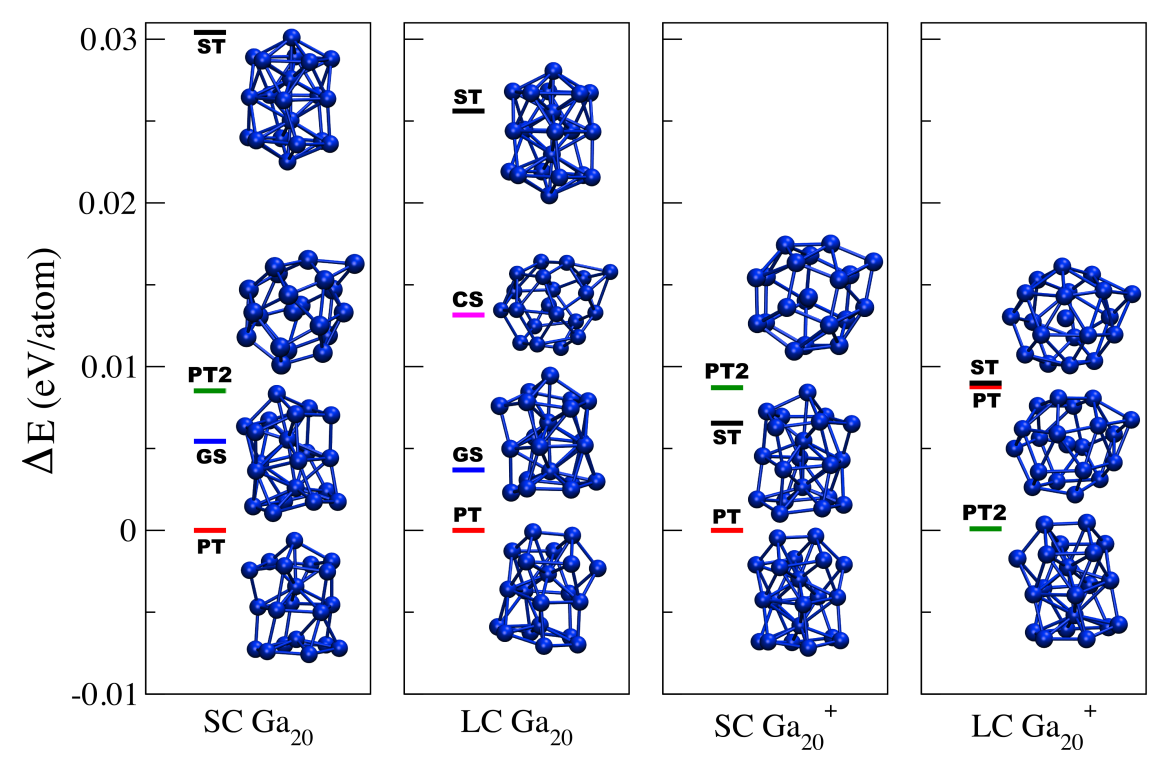

Figure 5.3. A relative binding energy diagram for each of the four simulations, illustrating the structural effects of parallel tempering. Binding energies were calculated using the GGA-PW91 functional with either the SC or LC gallium PAW potential, with the energy differences referenced to the most stable (lowest energy) structures explored in each simulation. ST = starting structure, $\mathbf{P T}=$ lowest energy structure from parallel tempering, GS = ground state structure from [50], and PT2 = structural transition structures observed in the course of parallel tempering. The relative cohesive energy for the capped-sphere structure (CS) optimized for the LC neutral case is also included for reference, as this was the only simulation that did not appear to explore the non-starting isomer. Atomic coordinates for each structure are given in Tab. B.6 and Tabs. B.8 through B.20. 


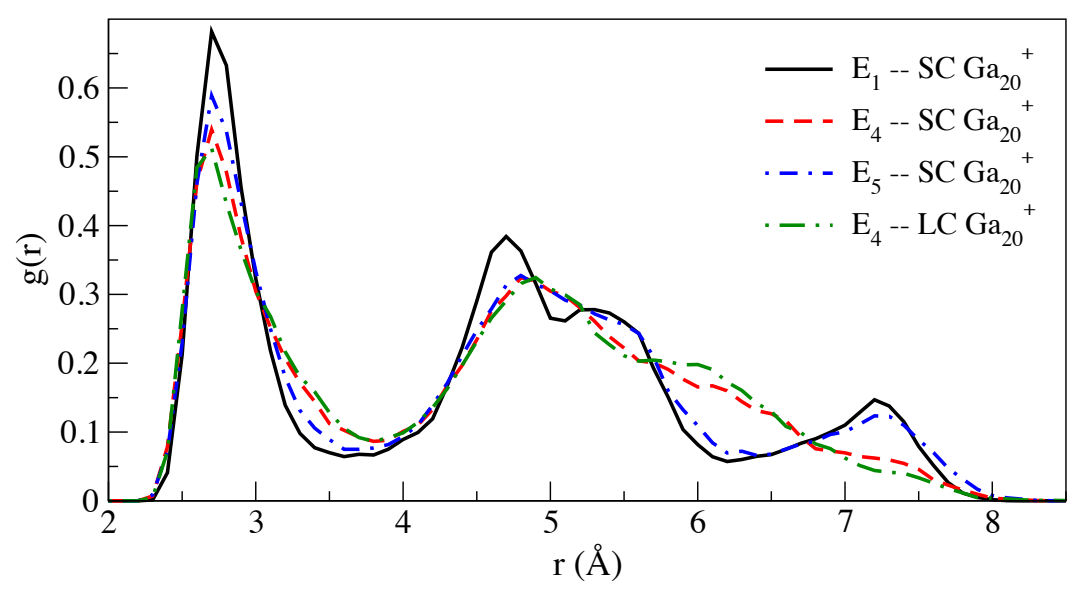

Figure 5.4. Normalized ARDF for the SC $\mathrm{Ga}_{20}^{+}$over 20 ps at the lowest energy ( $E_{1}$, solid black line), the fourth lowest energy where our simulations generate a structural transition to the capped-sphere structure $\left(\mathrm{E}_{4}\right.$, dashed red line), and the fifth lowest energy ( $E_{5}$, blue dash-dot line). It also includes the ARDF for 20 ps from the fourth lowest energy of the $\mathrm{LC} \mathrm{Ga}_{20}^{+}$ capped-sphere simulation (green long dash-dot line). 


\subsection{Convergence analysis}

For each simulation, the relative convergence of the microcanonical and canonical specific heat curves was analyzed using a sliding window analysis over a span of 10 consecutive time windows incremented in 1 ps intervals. The width of the window was held constant for each of the 10 consecutive windows and equaled the length of the microcanonical simulation less the microcanonical equilibration time. Quantitatively, the convergence criteria was set as: the temperature of the canonical specific heat curve peak changed by less than $10 \mathrm{~K}$ and the microcanonical specific heat peak changed by less than $0.01 \mathrm{eV} /$ atom over the 10 consecutive windows (10 ps). Qualitatively, the overall shape and general character of each specific heat curve had to remain consistent over the 10 consecutive windows.

Using these measures, Tab. 5.1 gives a summary of the key thermodynamic quantities and their relative convergence for each of the simulations. The simulation time listed is the total microcanonical simulation time per energy, excluding the 10 ps canonical equilibration. Fig. 5.5 is included as an illustrative example of both the quantitative and qualitative convergence parameters, showing the canonical and microcanonical specific heat curves over the 10 ps interval for the quantitatively least converged simulation.

\subsection{Thermodynamics}

Fig. 5.6 presents the canonical specific heat curves for each of the gallium simulations, normalized to the classical canonical specific heat accounting for rotational and vibrational contributions, $C_{0}=(3 N-6+3 / 2) k_{B}$. The overall nature of both the canonical specific heat curves as well as the melting temperatures are similar for clusters of the same charge. The nature of the pseudopotential (the inclusion of the $d$-electrons) has an evidently minor effect on the melting temperatures, although the latent heat is notably 


\begin{tabular}{lccccc} 
Cluster & $\mathrm{T}_{\mathbf{m}}$ & $\mathrm{E}_{\mathbf{m}}$ & $\Delta \mathrm{T}_{\mathbf{m}}$ & $\Delta \mathrm{E}_{\mathbf{m}}$ & Sim. Time \\
\hline $\mathrm{SC} \mathrm{Ga}_{20}$ & 733 & -2.079 & 0.662 & $4.45 \mathrm{e}-4$ & 92 \\
$\mathrm{LC} \mathrm{Ga}_{20}$ & 747 & -2.372 & 0.134 & $-9.96 \mathrm{e}-5$ & 93 \\
$\mathrm{SC} \mathrm{Ga}+$ & 611 & -1.810 & 0.663 & $4.69 \mathrm{e}-4$ & 166 \\
$\mathrm{LC} \mathrm{Ga}_{20}^{+}$ & 616 & -2.082 & -0.352 & $4.82 \mathrm{e}-5$ & 124
\end{tabular}

Table 5.1. Summary of the key thermodynamic quantities and convergence measures. The units are $\mathrm{K}$ for temperature, $\mathrm{eV} /$ atom for energy, and ps for simulation time. $\Delta \mathrm{T}_{\mathrm{m}}$ is the change per ps in the temperature of the canonical specific heat curve peak, given in $\mathrm{K} / \mathrm{ps}$, and $\Delta \mathrm{E}_{\mathrm{m}}$ is the microcanonical analogue in $\mathrm{eV} /$ atom/ps.
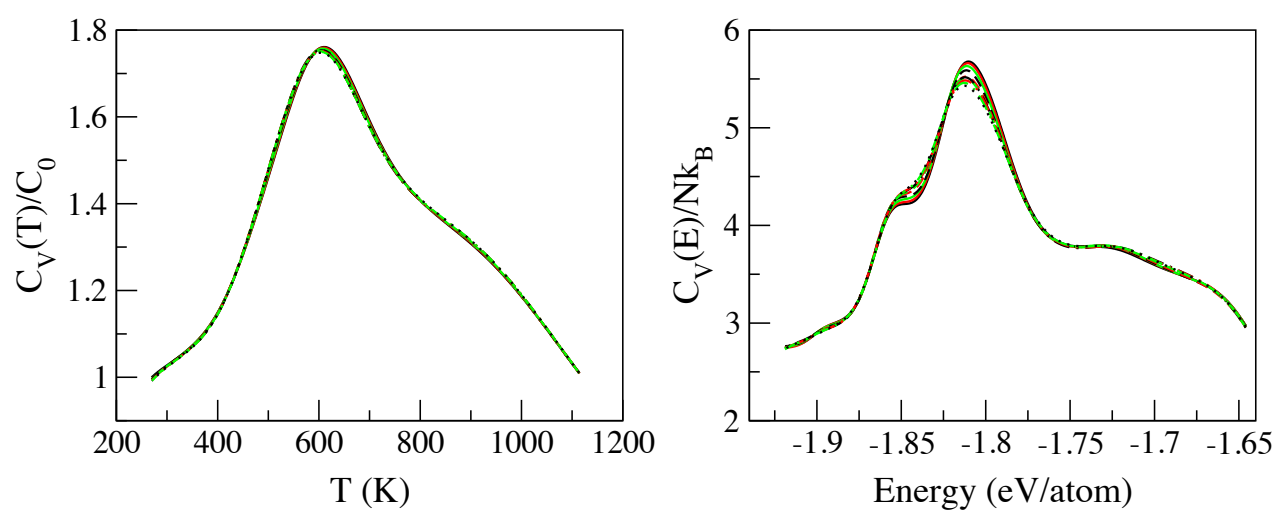

Figure 5.5. Normalized canonical (left) and microcanonical (right) specific heat curves for the 10 ps convergence interval for the quantitatively least converged simulation, the $\mathrm{SC} \mathrm{Ga}_{20}^{+}$.

larger for each SC simulation. The source of this pseudopotential effect on latent heat remains an open question.

Each of the neutral $\mathrm{Ga}_{20}$ specific heat curves exhibits a notable change in the slope around 550-600 K, followed by a smooth rise and fall surrounding melting temperatures of $733 \mathrm{~K}$ and $747 \mathrm{~K}$ for the SC and LC 


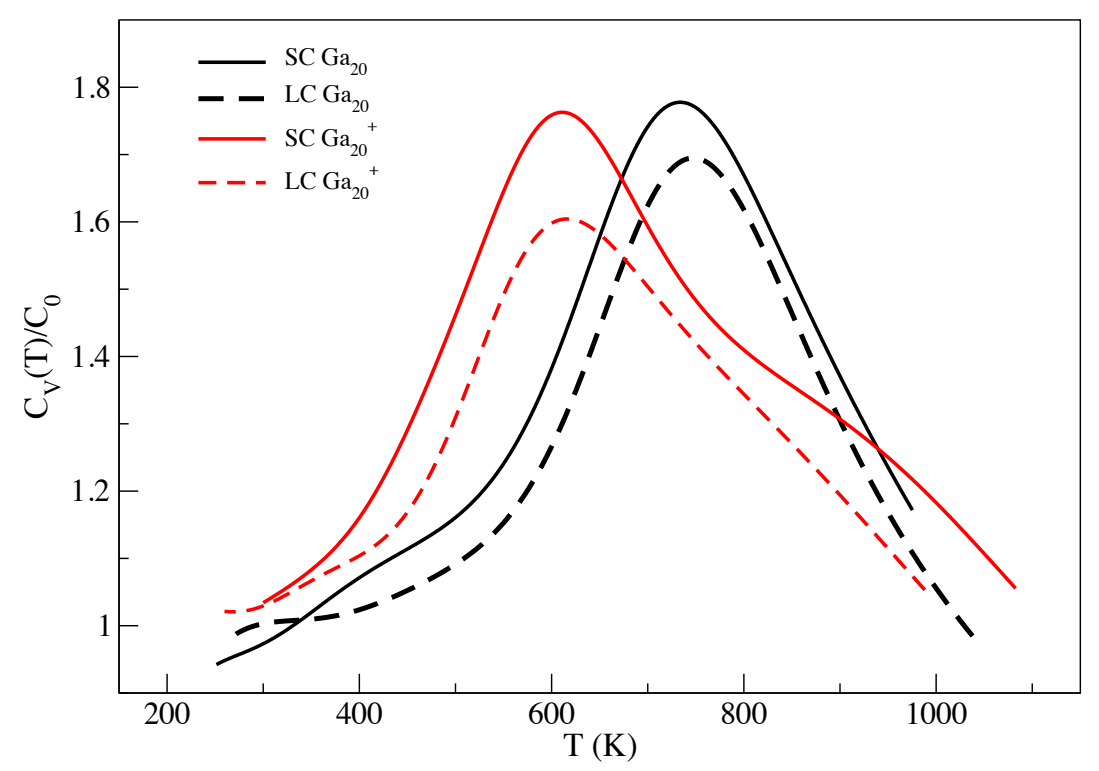

Figure 5.6. Normalized canonical specific heat curves for the SC $\mathrm{Ga}_{20}$ (black, solid), LC Ga $\mathrm{La}_{20}$ (black, dashed), $\mathrm{SC} \mathrm{Ga}+{ }_{20}^{+}$(red, solid) and $\mathrm{LC} \mathrm{Ga}_{20}^{+}$ (red, dashed) simulations.

simulation, respectively. Both $\mathrm{Ga}_{20}^{+}$simulations have a considerably lower melting temperatures, with a peak at $611 \mathrm{~K}$ and $616 \mathrm{~K}$ for the SC and LC models, respectively, with broader peaks than for the neutral clusters. While the neutral simulations better capture the experimental melting temperature, the cation simulations better capture the general nature of the experimental specific heat curve. As a point of interest, Fig. 5.7 includes a previous simulation of $\mathrm{Ga}_{20}^{+}$completed by Krishnamurty et. al. using the large core, ultrasoft pseudopential in the canonical ensemble [32]. While their study was completed for the 20-atom gallium cation, their results more closely match our LC neutral simulation. Fig. 5.7 also includes the experimental results for $\mathrm{Ga}_{20}^{+}$[32]. Noting approximate shifts of $+0.22 C_{V}(T) / C_{0}$ and $-110 \mathrm{~K}$ from the experimental results, the $\mathrm{LC} \mathrm{Ga}_{20}^{+}$ simulation captures the overall shape of the $C_{V}$ curve quite well. 


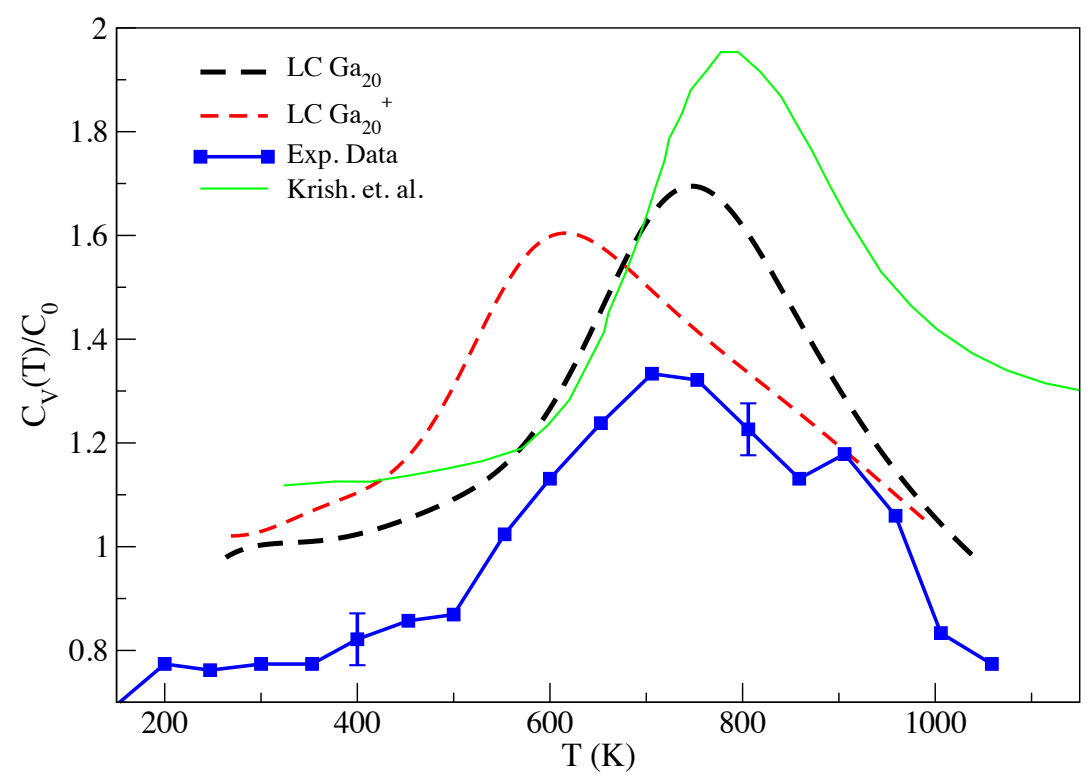

Figure 5.7. Normalized canonical specific heat curves for the LC PAW pseudopotential simulations of $\mathrm{Ga}_{20}$ (black, dashed) and $\mathrm{Ga}_{20}^{+}$(red, dashed), contrasted with the large core, ultrasoft pseudopotential (green solid) simulation of $\mathrm{Ga}_{20}^{+}$completed by Krishnamurty et. al. [32]. The experimental data (blue with square markers) is included for reference [32].

The quantitative agreement between the calculated melting temperatures of the clusters and the experimental values is reasonable. It is already apparent that a single electron can shift the melting temperature by more then $100 \mathrm{~K}$, which goes some way to explaining the extreme size-dependence of cluster melting temperatures. It should be noted that Starace et. al. experimentally observed similar one-electron size dependencies for certain sizes of aluminum clusters [57]. Often, these one-electron differences were related back to significant ground state structural differences between the different charge states (anion, neutral or cation) of a given cluster size. However, for certain sizes, e.g. $A l_{35}$, a significantly higher melting temperature is observed for the anion compared to the neu- 
tral and cation clusters while no difference in the ground state structures was noted. This study concludes that such melting temperature differences must arise from an energetic origin relating to electronic stabilization, as $A l_{35}^{-}$corresponds to a jellium-like electronic shell closing at 106 electrons. For our research, neither $\mathrm{Ga}_{20}$ nor $\mathrm{Ga}_{20}^{+}$exhibit a jellium closed shell electronic structure. The stacked-plane and capped-sphere structures observed during the course of the cationic simulations represent competing low-energy isomers, as opposed to a structural transition. While it is possible that the origin of $100 \mathrm{~K}$ melting temperature variation between $\mathrm{Ga}_{20}$ and $\mathrm{Ga}_{20}^{+}$could arise from a significant ground state geometric difference between the two charge states, this difference was not observed in the course of these simulations, even with the use of parallel tempering. It is also worth noting that an extensive study investigating the global minimum structures for small neutral and charged gallium clusters identified the same ground state structures for $\mathrm{Ga}_{20}$ and $\mathrm{Ga}_{20}^{+}$[46].

The microcanonical specific heat curves are presented in Fig. 5.8. The differing PAW potentials yielded different energy ranges for the simulations making any comparison of melting energies problematic. While each of the microcanonical specific heat curves exhibit unique characteristics, there is again notable similarity between clusters of the same charge state. The SC cation has a shoulder on the low-energy side of the peak at -1.85 $\mathrm{eV} /$ atom, which has been attributed to the stacked-plane/capped-sphere transition observed during the parallel tempering swapping.

It is interesting to note that although the ARDF's for the SC $\mathrm{Ga}_{20}$ and $\mathrm{SC} \mathrm{Ga}_{20}^{+}$clusters are nearly identical, they exhibit significantly different melting signatures. It would be tempting to attribute this similarity to the lowest-energy PT-derived structures (Fig. 5.3), which are of the same structural class for these two simulations but exhibit a modification to the stacked-planes, with the neutral cluster stacking as 1-6-1-5-1-6 atoms while the cation has planes of 2-5-1-5-1-6 atoms. However, the LC and SC $\mathrm{Ga}_{20}^{+}$simulations identify dramatically different ARDF's (at energies be- 

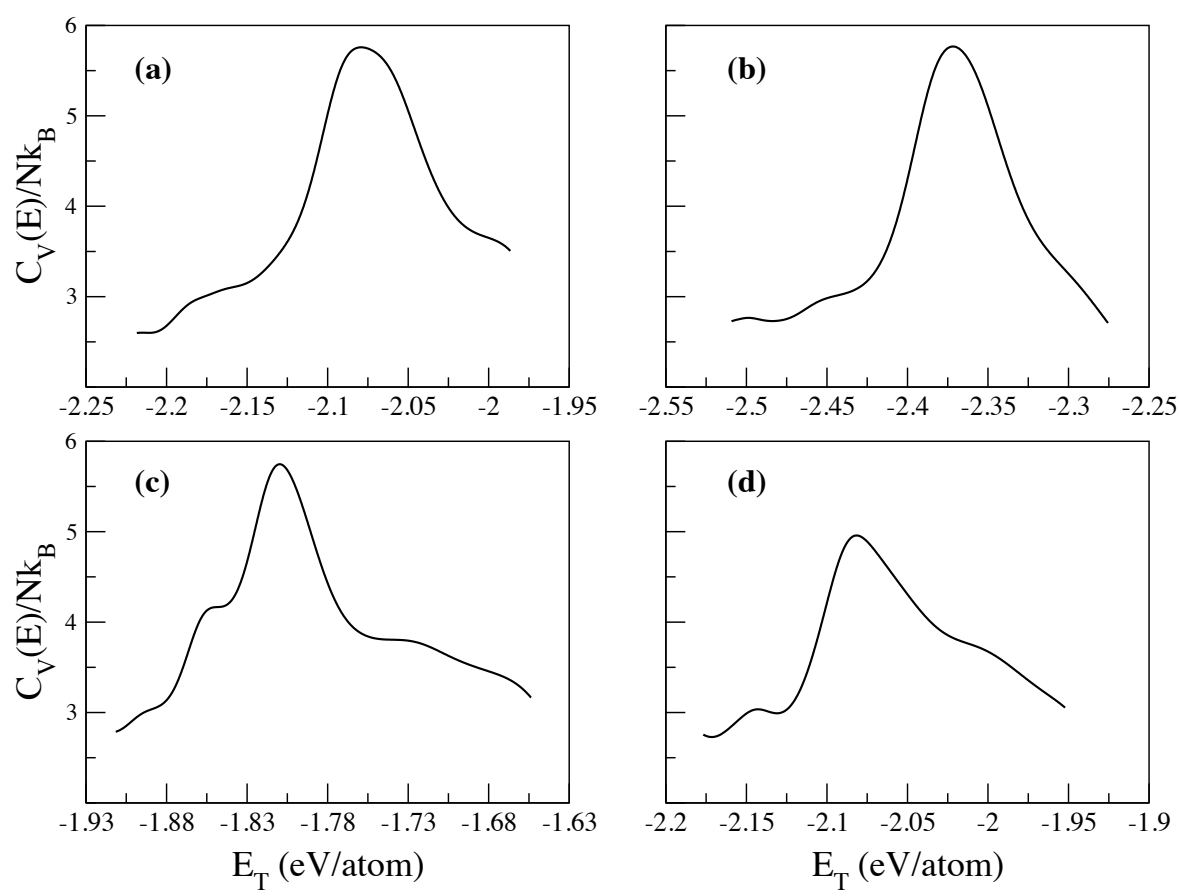

Figure 5.8. Microcanonical specific heat (calculated by the multiple histogram method) as a function of total energy per atom for the (a) $\mathrm{SC} \mathrm{Ga}_{20}$, (b) $\mathrm{LC} \mathrm{Ga}_{20}$, (c) $\mathrm{SC} \mathrm{Ga}+20$ and (d) $\mathrm{LC} \mathrm{Ga}_{20}^{+}$simulations.

low melting), yet their canonical specific heat curves are strikingly similar. While PT does identify the stacked-plane isomer in the $\mathrm{LC} \mathrm{Ga}_{20}^{+}$simulation, it persists for a relatively short period of time and the ARDF clearly demonstrates that the simulation is dominated by its initial capped-sphere structure.

In order to further investigate the finite temperature behavior of each cluster and the structural contributions to melting, we exploit the statistical structural information available from MD simulations as addressed in the following section. 


\subsection{Structural dynamics}

The results of the Lindemann Index analyses are given in Fig. 5.9, illustrating $\delta_{r m s}$ as a function of average temperature. By the traditional Lindemann criteria for a solid-liquid phase transition of $0.1-0.15$, all our clusters would be melted at the very lowest energy. Considering both the atomic structure and specific heat analyses, however, it is likely these high initial values are due solely to the greater mobility (lower rigidity) of small clusters. It is interesting to note that $\delta_{r m s}$ for the charged clusters is consistently greater than either neutral cluster, suggesting that the cations have less overall rigidity. This greater mobility could contribute to the lower melting temperatures for the cations. The cations also show a smoother increase of $\delta_{r m s}$ with increasing temperature. This more gradual progression helps to explain the broader specific heat curves observed for the cations with the only variance of the cluster bond length increasing gradually, causing the phase transition to spread over a wider temperature range.

A clear, steep rise in the Lindemann index, which is typically considered indicative of a phase transition, is not observed for any of our simulations. However, as noted in Sec. 4.5.2, the values of $\delta_{r m s}$ within $\sim 100 \mathrm{~K}$ to each side of the phase transition should be taken with caution. We do, however, see convergence of the curves at higher energies. We have annotated the temperature of the peak in the canonical specific heat curves (from Fig. 5.6) by the vertical dashed lines in Fig. 5.9. While the discrete nature of the $\delta_{r m s}$ values coupled with the averaging of temperature per sub-run accounts for a large margin of error, we find the simulated melting temperature can be closely affiliated with the onset of $\delta_{r m s}$ convergence.

Finally, we compute the atomic mean square displacements (MSD) for each cluster. Fig. 5.10 gives the MSD for each of the simulations. For each of the stacked-plane simulations ( $\mathrm{SC} \mathrm{Ga}_{20}, \mathrm{LC} \mathrm{Ga}_{20}$ and $\mathrm{SC} \mathrm{Ga}_{20}^{+}$), the displacements for the two central, negatively charged atoms were averaged 
separately from the other 18 surface atoms. For the $\mathrm{LC} \mathrm{Ga}_{20}^{+}$capped-sphere simulation, the MSD for the central and capping atom were distinguished from the average of the remaining 18 surface atoms. Generally speaking, the fluctuations in the individual atomic mobilities increased with increasing constant energy. As illustrated in Fig. 5.10, however, the central atoms have significantly lower mobilities than the surface atoms for all clusters. While this low mobility persists even at higher energies, the central atoms become equally mobile to the surface atoms at energies above the melting point. Additionally, the average mobilities of the surface atoms remain relatively constant across each simulation's energy range, increasing slightly for energies above melting. At low energies, the central atoms serve as an anchor about which the other atoms oscillate, with the distinction between surface and interior atoms being a key signature of solidity which disappears as the cluster melts. As noted in Sec. 4.5.3, the exact values of MSD in the region of the phase transition should be taken with caution.

While the greater structural rigidity of the neutral clusters likely contributes to the $100 \mathrm{~K}$ melting temperature increase relative to the cation, the origin of this difference remains an open question. The general structural similarity between the cationic and neutral clusters, however, suggests that electronic structure plays a significant role in the thermodynamic behavior of the 20-atom gallium clusters.

\subsection{Electronic structure}

In order to discern the electronic structure contributions to melting, three measures were utilized: Bader analysis [148], electron localization analysis $[152,153,154]$. The simplest comparison to be made between the neutral and charged clusters is the distribution of charge. We therefore consider the lowest-energy gallium structures identified in our simulations (PT from Fig. 5.3), and employ Bader analysis. Fig. 5.11 shows the distribution of charge, which remains consistent between the neutral and 


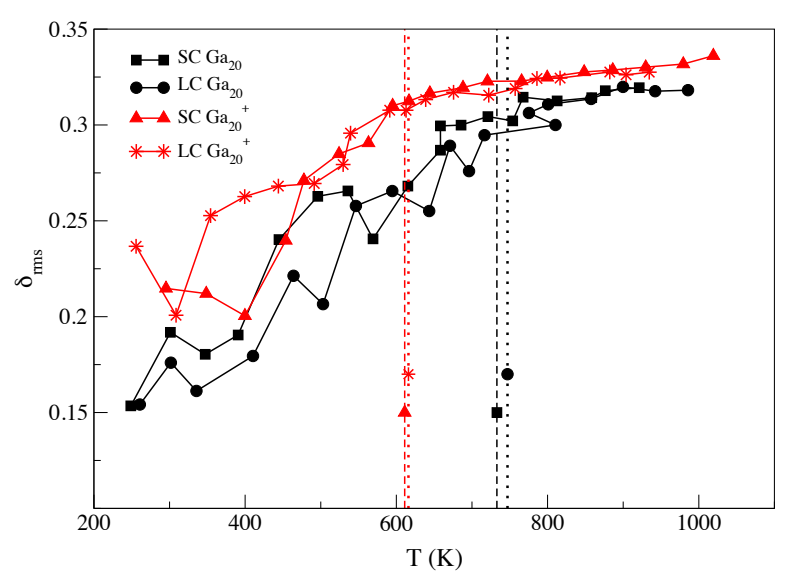

Figure 5.9. Lindemann index, $\delta_{r m s}$, versus the average temperature for each sub-run. The higher mobility for the charged simulations (red) versus the neutral simulations (black) is clearly evident at all energies. The vertical dashed lines represent the temperature of the peak in the canonical specific heat curves (Fig. 5.6).

charged clusters: the central atoms in both structural classes are negatively charged while the surface atoms are slightly positive or neutral.

For the two different structures analyzed for the cation, some small differences are noted. The SC $\mathrm{Ga}_{20}^{+}$stacked-plane structure contains planes exhibiting the same magnitude of charge, while in the $\mathrm{LC} \mathrm{Ga}_{20}^{+}$cappedsphere structure, the negative charge is isolated on the central atom, with the most external atom positively charged. It should be emphasized that although this spherical structure looks highly symmetric, the distance between the central atom and the surrounding sphere is very large, at $3.25 \AA$, as compared to the inter-sphere pair bonds at $\sim 2.8 \AA$. In the two neutral stacked-plane structures, the two central atoms, though reminiscent of the pairing of atoms in $\alpha-\mathrm{Ga}$, are too far apart to be considered a dimer at between 3.17 and $3.25 \AA$. For the SC $\mathrm{Ga}_{20}^{+}$cluster, the two central atoms have a bond of $2.85 \AA$, which is still $0.2 \AA$ longer than the hexagonal/pentagonal 

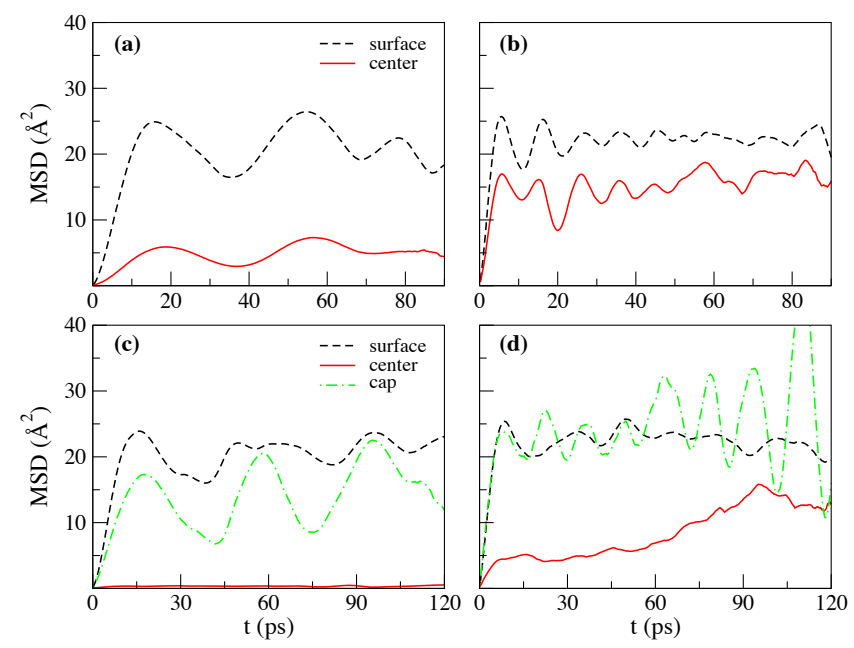

Figure 5.10. Atomic MSD's averaged over the surface atoms (black, dashed), central atom(s) (red, solid) and capping atom (green, dash-dot) for the stacked-plane $\mathrm{LC} \mathrm{Ga}_{20}$ simulation (top row) and capped-sphere LC $\mathrm{Ga}_{20}^{+}$simulation (bottom row) at the lowest energy (a and c) and an energy just below the phase transition ( $b$ and $d$ ). As noted in Sec. 4.5.3, MSD measures should be taken with caution at phase transition temperatures represented by (b) and (d).

intra-planar pair bonds at $\sim 2.65 \AA$. Thus, we observe a structural distinction between the internal and surface atoms for both structural isomers.

The centralized accumulation of electron density can be understood by an analysis of the spherical harmonic-projected electronic density of states (SH-PDOS, as calculated in previous work [149]). The SH-PDOS for each of the PT-lowest energy structures is illustrated in Figs. 5.12 and 5.13 for the neutral and cationic clusters, respectively. In the neutral case, a clear progression of $1 S, 1 P, 1 D \ldots$ states can be seen at low energies, with a splitting of the peaks due to the two central atoms in the stacked-plane structure. At around the HOMO-LUMO gap, the clear angular momentum character of the peaks is diminished. In the cationic case, a marked dif- 


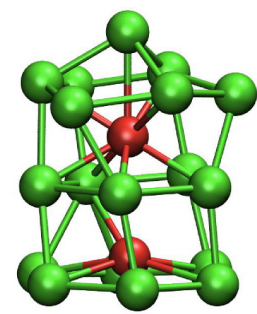

(a)

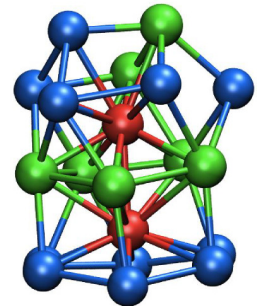

(c)

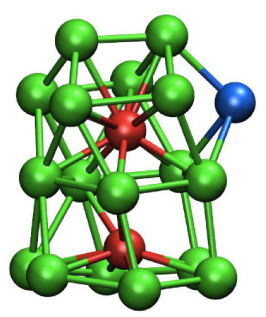

(b)

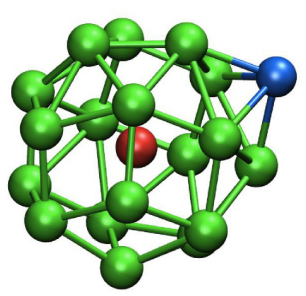

(d)

Figure 5.11. Bader analysis for the (a) SC $\mathrm{Ga}_{20}$, (b) $\mathrm{LC} \mathrm{Ga}_{20}$, (c) $\mathrm{SC} \mathrm{Ga}_{20}^{+}$ and (d) $\mathrm{LC} \mathrm{Ga}_{20}^{+}$lowest energy structures identified in parallel tempering simulation. In order of most negative to most positive charge, with partial charge: (red) less than $-0.2 e$; (green, $\sim$ neutral) between $-0.1 e$ and $0.1 e$; and (blue) greater than $0.2 e$.

ference can be seen between the stacked-plane structure and the cappedsphere. The centro-symmetric nature of the capped-sphere isomer provides a much clearer spherical potential in which to see the electronic shell structure. At the HOMO-LUMO gap there is a distinct change from $1 G$ to $2 D$ character, corresponding to the closed shell structure expected at 58 electrons (shell structure occupancy of $1 S^{2}, 1 P^{6}, 1 D^{10}, 2 S^{2}, 1 F^{14}, 2 P^{6}, 1 G^{18}$ ). The HOMO contains the 59th electron and is of no obvious angular momentum character.

Analysis of the electron localization function can help complete our understanding of these effects. Covalent bonds can be identified in the ELF by a region of electron localization located on the radial axis between two ions, approximately equally spaced and distinctly separate from the 


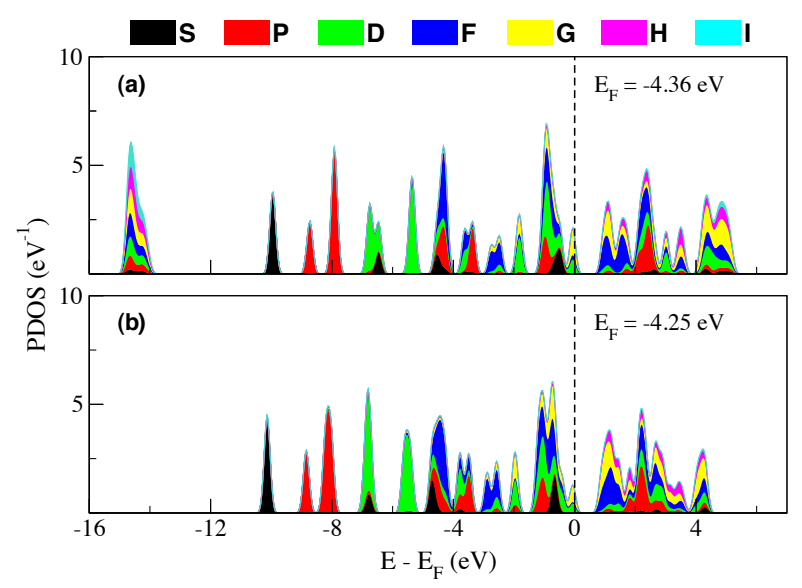

Figure 5.12. Spin-up contribution to the SH-PDOS for the (a) $\mathrm{SC} \mathrm{Ga}_{20}$ and (b) $\mathrm{LC} \mathrm{Ga}_{20}$ lowest energy structures identified in each parallel tempering simulation. For both plots, the SH-PDOS are calculated within a cut-off radius of $3.0 \AA$ from the cluster's center of mass.

ionic cores. This distinction is made from an ELF-identified ionic bond in which the electron localization is more closely associated with one or the other ionic core. As shown in Fig. 5.14, the electron localization analysis for each of the four clusters shows no identifiable covalent bonds. Instead, we observe atom centered electron localization, which may overlap for sufficiently low values of the isosurface, but which does not identify a covalent bond. Thus, in contrast to previous studies on melting small gallium clusters $[45,32]$, we find no evidence of covalency in our analysis of the bonding in these clusters. The electronic shell structure instead implies a metal-like character to the bonding, with delocalization of the electron density over the cluster only perturbed by the positions of the atomic cores. This is consistent with the findings of Núnez et al. that the electronic structure of small cationic, neutral and anionic gallium clusters is essentially metallic [46].

The SC $\mathrm{Ga}_{20}$ and SC $\mathrm{Ga}_{20}^{+}$clusters look very similar in all electronic and atomic structural measures, yet we have observed distinctly different 


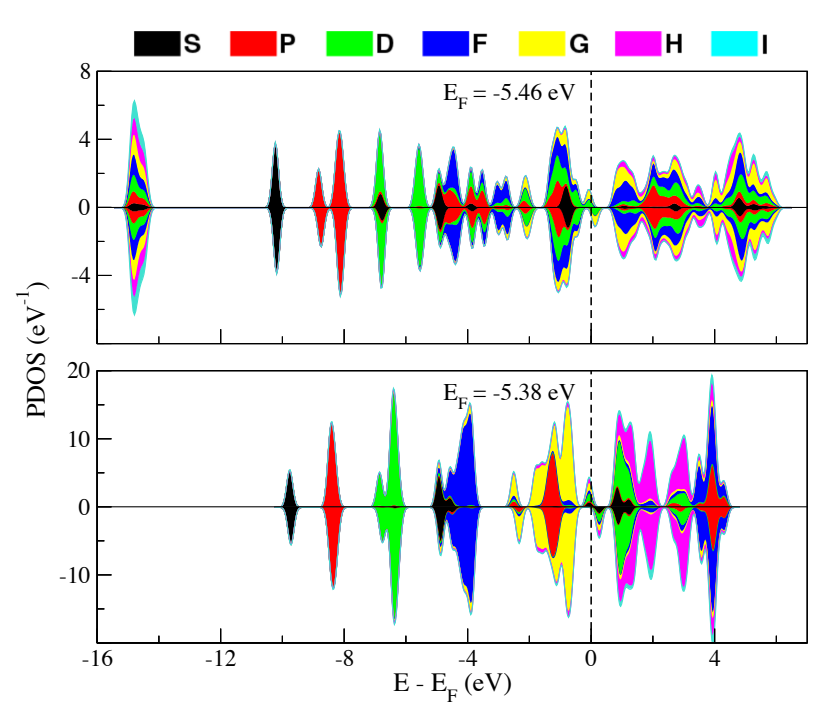

Figure 5.13. Spin-up and spin-down contributions to the SH-PDOS for the (a) SC $\mathrm{Ga}_{20}^{+}$and (b) $\mathrm{LC} \mathrm{Ga}_{20}^{+}$lowest energy structures identified in each parallel tempering simulation. The SH-PDOS is calculated within a cut-off radius of (a) $3.0 \AA$ and (b) $6.0 \AA$ from the cluster's center of mass.

melting behavior. While there is a clear distinction in SH-PDOS for the LC ion capped-sphere and SC ion stacked-plane structures, it likely arises from their distinct atomic structures which, given the similarity of their simulated melting behavior, does not appear to alter the thermodynamic behavior. The potential energies of the neutral clusters are lower than those of the cationic clusters, making the neutral cluster more strongly bound. However, as melting properties are related to the stabilities of both the solid and liquid phases, we have also investigated the potential energies of the liquid clusters at the melting temperature. For both pseudopotential models, the energy difference between the solid and liquid phase is larger for the neutral cluster, which correlates with its higher melting temperature. However, from Bader, SH-PDOS and ELF analysis, we see no clearly distinguishable differences in the electronic structure related to 


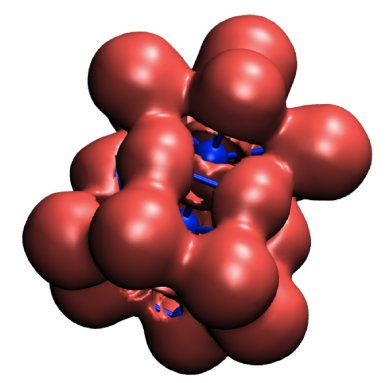

(a)

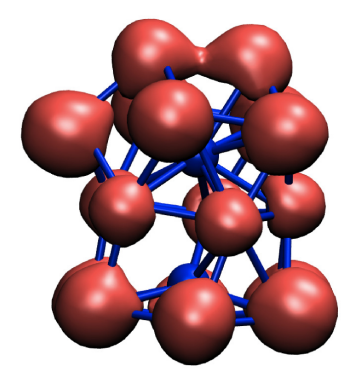

(c)

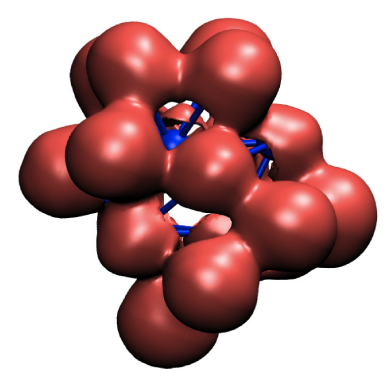

(b)

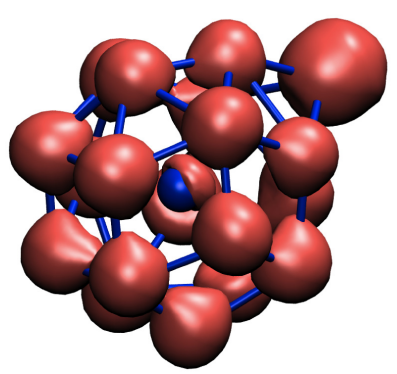

(d)

Figure 5.14. ELF plots for the lowest energy structures identified in parallel tempering. (a) SC $\mathrm{Ga}_{20}$ and (b) SC $\mathrm{Ga}_{20}^{+}$clusters at ELF $=0.2$, illustrating the marked similarity of their electron localization. (c) LC $\mathrm{La}_{20}$ and (d) LC $\mathrm{Ga}_{20}^{+}$at $\mathrm{ELF}=0.6$, illustrating the lack of covalency. The difference in the cutoff ELF values between the SC and LC clusters is due to the different number of valence electrons for each model.

bonding that would contribute to this increased neutral stability, indicating that the influence of electronic structure on melting is a subtle effect and will require further investigation.

\subsection{Summary}

Four DFT-based simulations of the melting phase transition were completed for the 20-atom gallium cluster. Using a parallel tempering algo- 
rithm in order to speed convergence and augment the exploration of configurational phase space, we demonstrate that we discover lower energy configurations than the initial structures over the course of the simulation. With two distinct core potentials, we illustrate that the valence treatment of the d-electrons has no significant impact on the modeled melting temperature, while the charge state has a notable effect on both. The pseudopotential model does have a significant effect on the latent heats of the simulations, the origin of which remains an open question for future research.

Comparing our modeled canonical specific heat curves to the experimental data [32], we see that the LC $G a_{20}^{+}$simulation most closely matches the overall nature of the experimental curve while experimental melting temperature is best reproduced by the neutral cluster simulations. The discrepancy between experimental and calculated melting temperatures is most likely due to limitations of the density functional description.

The Lindemann index analyses yield further evidence of a phase transition for 20-atom gallium neutral and charged clusters, with the onset of a smooth progression of $\delta_{r m s}$ correlating well to the temperature of the specific heat curve maximum. This analysis also illustrates that, as compared to the neutral clusters, cations have an overall higher atomic mobility and display a smoother increase in the Lindemann index with increasing energy. These differences, along with the lower cohesive energy, could account for both the lower melting temperature and wider melting transition of the cationic clusters, warranting further investigation with additional cluster sizes.

In order to examine the nature of bonding previously implicated in greater-than-bulk melting temperatures, we have combined Bader analysis, SH-projected electronic DOS, and ELF analysis. No difference in electronic structure, beyond electron number, could be identified between SC $G a_{20}$ and $G a_{20}^{+}$clusters in order to explain the thermodynamic variation. While ELF analysis indicates the role of charge polarization between cen- 
tral and outer atoms, there is no sign of covalent bonding as has been previously reported. The SH-projected electronic DOS demonstrates the persistence of electronic shell structure and provides some insight into the differences between the observed cluster structures and the structures known in the bulk. Electronic shell structure increases the effective volumes of the central atoms at these sizes, as shown by the central-to-outer atomic bond distances as compared to outer-to-outer atomic bond distances. Further investigation will be required in order to determine if this increase serves to strengthen the interactions between surface atoms, which would partially explain the higher-than-bulk melting temperatures. MSD analysis yields further evidence of this strengthening in the notably lower atomic mobilities for the central atoms, indicating that the outer atoms form a tight cage within which the central atoms are trapped.

Although the SC neutral and cationic clusters share nearly identical ARDF's over the range of energies, they exhibit distinct melting temperatures. The shape of the specific heat curves and RMSD analyses are correspondingly different. While the SC and LC cationic clusters have entirely distinct ARDF's at energies below melting, their specific heat curves and RMSD's are markedly similar. These two comparisons clearly indicate that electronic structure is important to the nature of thermodynamic behavior in these clusters and warrants further investigation. In order to further our understanding of the phase transitions in these small systems, simulations were completed for a set of smaller gallium clusters, sized 7-12 atoms. The small size of these "microclusters" greatly simplifies the system, allowing for a clearer exploration of the atomic and electronic structure contributions to small gallium cluster thermodynamics. The results of these simulations are detailed in the following chapter. 


\section{Chapter 6}

\section{Microclusters}

Simplifying the system and further probing small cluster thermodynamics, FPMD melting simulations were completed for a range of very small gallium clusters, or microclusters, sized 7-12 atoms. These simulations probe both the structural contributions to greater-than-bulk melting as well as the size-limits on the very definition of what can be thermodynamically defined as melting. The following sections outline these results, giving an overview of the thermodynamic, atomic structure and electronic structure analysis for each cluster size.

\subsection{Background}

How does one define melting when the size of the system diminishes to a handful of atoms? Melting in the bulk phase is well-defined, with a transition from an ordered, rigid structure to a less ordered structure lacking a fixed shape. Practically speaking, the bulk melting temperature can be defined by a sharp rise in the specific heat or change in a macroscopic measure of order, such as the electron diffraction pattern disappearing when the temperature is raised.

Macroscopic measures break down as the system size diminishes to 10 's of atoms, as clusters in this size regime often lack well-defined sym- 
metry or shape. Significant advances in technology, however, have enabled accurate experimental specific heat measurements of incredibly small clusters [163]. The experiments, outlined in greater depth in Sec. 1.1.3, have revealed a number of counterintuitive properties for these small clusters, such as greater-than-bulk melting temperatures for tin and gallium clusters [29, 25, 30, 31, 32], intriguingly strong size-sensitivity [35] and enhanced reactivity $[164,165,166]$.

While the sharp rise in the bulk specific heat is broadened due to finite size effects [163], these experiments have clearly identified specific heat peaks associated with a solid-to-liquid phase transition for a range of small cluster sizes. As the size of the clusters diminish, however, the latent heat of melting also decreases [167] and the phase transitions become less well-defined $[25,35]$. Experimental specific heat measurements for the smallest sizes include $\mathrm{Al}_{16}^{+}$[27] and $\mathrm{Ga}_{17}^{+}$[25], which exhibit rather featureless specific heat curves without clearly defined melting transitions. For the smallest aluminum cluster sizes measured, some evidence indicated that the melting temperatures might have exceeded the measured range [27]. However, this same study noted that the measurements become more challenging with decreasing cluster size, as the temperatureinduced change in internal energy diminishes [27]. In fact, for clusters of $\sim 10$ atoms or less, the very definition of melting could be questioned. This chapter addresses the question of how melting can be defined and identified in such small systems.

The fact that experimental reports are lacking for $\mathrm{n}<16$ atoms has not hindered theoretical probing. Research investigating the global minimum (GM) for 2-12 atom clusters of sodium [145], tin [168], gallium [48, 49, 50] and aluminum [169] has revealed an intriguing odd-even motif in several properties, such as cohesive energy second order differences, average coordination number, nearest-neighbor distances and HOMO-LUMO gap. Melting simulations for microclusters, or clusters sized 2-12 atoms, have been more limited. Classical and first-principles molecular dynamics sim- 
ulations have been completed for microclusters of sodium $[146,56,52,53$, 141], argon [170, 171, 144, 140], gold [172], silver [173], lithium [174], tin [175], and aluminum [176]. However, aside from the classical MD simulations completed by Werner et al. [176], each of these studies investigates individual sizes, without addressing how melting changes across a size range. First-principles melting simulations systematically investigating thermodynamic changes across a size range of microclusters have never been completed.

Probing the limits of melting with a systematic approach to size, we present the results of a series of parallel tempering first-principles molecular dynamics (FPMD) simulations for gallium clusters sized 7-12 atoms. This work extends and combines previous research on the GM structures for small gallium clusters [49] and greater-than-bulk melting [161] to include the thermodynamic properties of microclusters, exploring the limits of FPMD melting. The following sections outline the initialization of the simulations and the effects of parallel tempering, as well as provide details of the specific heat, root-mean square bond length variance, mean square displacements and electronic structure analysis completed in order to further our understanding of small cluster thermodynamics.

\subsection{Microcluster Simulations}

Geometry optimizations were completed for each neutral cluster using the LC PAW potential and the same VASP settings outlined in Section 5.1. Figure 6.1 gives an overview of each optimized cluster structure. With the exception of the 12-atom structure, the structures seeded to each geometry optimization were derived by systematically removing atoms from the upper-half of the PT-derived lowest-energy structure for the SC Ga $\mathrm{Ca}_{20}^{+}$simulation, as illustrated by PT in the third column of Fig. 5.3. The initial optimization for $\mathrm{Ga}_{12}$ was seeded with a symmetric icosahedron.

Each optimized initial structure is shown in Fig. 6.1. Although the op- 
timizations for $\mathrm{Ga}_{7}$ and $\mathrm{Ga}_{8}$ were initialized with pentagonal/hexagonal plane-like structures, the optimizations yield the rhomboid-like structures. It should also be noted that the optimized $\mathrm{Ga}_{8}, \mathrm{Ga}_{11}$ and $\mathrm{Ga}_{12}$ structures are highly symmetric, with the 12-atom cluster demonstrating a geometric icosahedral shell closing. For the 11- and 12-atom simulations, the canonical equilibrations were restarted with lower energy structures derived from short microcanonical simulations. This was necessary in order to set the equilibrations to sufficiently low temperatures to cover the temperature range of interest. Although each initial structure served as a low-energy starting point for the simulations, it was not assumed that any represented the ground state configurations, relying on the parallel tempering algorithm to allow for an adequate sampling of configurational space.

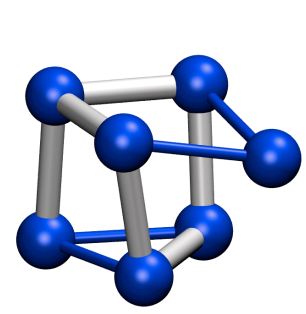

(a) $\mathrm{Ga}_{7}$

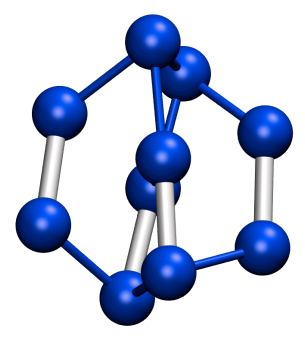

(d) $\mathrm{Ga}_{10}$

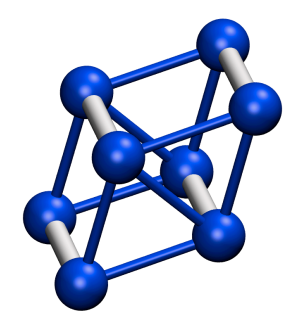

(b) $\mathrm{Ga}_{8}$

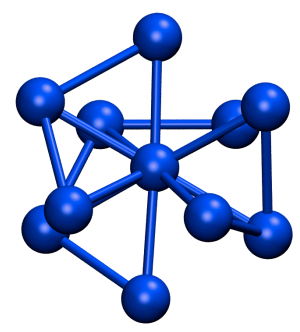

(e) $\mathrm{Ga}_{11}$

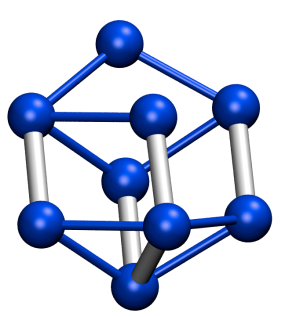

(c) $\mathrm{Ga}_{9}$

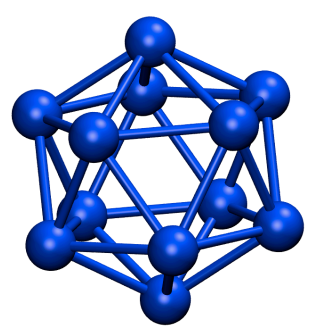

(f) $\mathrm{Ga}_{12}$

Figure 6.1. Optimized initial structures for the clusters sized 7-12 atoms, with $r_{12}<2.6 \AA$ in grey and $2.6 \AA<r_{12}<2.8 \AA$ in blue. Atomic coordinates for each small cluster initial structure are given in Tabs. B.21 through B.26. 


\begin{tabular}{cccc}
\hline \hline Cluster & $\mathbf{E}_{\mathrm{Geo}}$ & $\mathbf{E}_{\mathbf{P T}}$ & $\boldsymbol{\Delta} \mathbf{E}$ \\
\hline $\mathrm{Ga}_{7}$ & 2.039 & 2.041 & 0.002 \\
$\mathrm{Ga}_{8}$ & 2.111 & 2.111 & - \\
$\mathrm{Ga}_{9}$ & 2.071 & 2.079 & 0.008 \\
$\mathrm{Ga}_{10}$ & 2.143 & 2.143 & - \\
$\mathrm{Ga}_{11}$ & 2.111 & 2.176 & 0.065 \\
$\mathrm{Ga}_{12}$ & 2.217 & 2.220 & 0.003 \\
\hline \hline
\end{tabular}

Table 6.1. Summary of the cohesive energies for the optimized initial structure $\left(\mathrm{E}_{G e o}\right)$ and lowest energy parallel tempering discovered structure $\left(\mathrm{E}_{P T}\right)$. Energies are in $\mathrm{eV} /$ atom.

The microcanonical FPMD parallel tempering implementation was identical to that outlined in Sec. 5.1. Given the similar thermodynamic results for the LC and SC pseudopotentials for the 20-atom cluster, the simpler LC model was utilized for microcluster simulations. For each cluster size, the lowest energy structure obtained during each PT simulation was optimized using the same settings as the initial optimizations, as outlined in Sec. 5.1, for purposes of comparison. The cohesive energies for the PTderived structures, calculated according to Eq. (5.1), are given relative to the cohesive energies of their initial structures in Tab. 6.1.

For the 8- and 10-atom clusters, the PT-derived structures are nearly identical to the initial structures with similar cohesive energies, while the 9-, 11- and 12-atom structures are entirely distinct with notably higher cohesive energies. The lowest energy PT-derived structure for $\mathrm{Ga}_{7}$ is similar to its initial seed, with slight bond length and structural modifications giving it a slightly lower energy. The lowest energy PT-derived structures for the $\mathrm{Ga}_{7}$ and $\mathrm{Ga}_{9}$ are asymmetric, while the other four clusters exhibit welldefined symmetry. For each of the PT-derived structures with a notable geometry change from its initial configuration, the initial and PT-derived structures are illustrated in Fig. 6.2. 
The lowest energy PT structure for the 8-atom cluster, as shown in Fig. $6.1(b))$, is a highly symmetric rhomboid. This structure is most reminiscent of bulk gallium, with the shortest pair bonds at $\sim 2.5 \AA$ and the next shortest at $2.7 \AA$. It is interesting to note that the PT-derived structures for $\mathrm{Ga}_{9}$ is significantly less symmetric than its initial seed structure. The PT-derived structure for the 9-atom cluster adds a single atom to the $\mathrm{Ga}_{8}$ rhomboid, disrupting the symmetry. For $\mathrm{Ga}_{11}$, the PT-derived structure has an intriguing butterfly symmetry, resembling two asymmetric $\mathrm{Ga}_{8}$ rhomboids sharing 5 central atoms. A distorted rhomboid can also be identified in the 10- and 12-atom structures. Although the rhomboid is significantly twisted for the 10-atom structure as shown in Fig. 6.1 (d), each of the four short (grey) bonds would correlate to those in the 8-atom cluster. For the 12-atom cluster, the short bond in Fig. 6.2 (d) represents one of the rhomboid edges.

As a final point of comparison, each of the PT-derived cohesive energies and 2nd order differences are plotted versus the number of atoms in Fig. 6.3. Although for a shorter size range, the general pattern for the cohesive energies matches well with those observed in previous research $[48,49,50,46]$. A similar odd/even oscillation with the second order differences is also noted, showing relatively higher stability for the even-numbered clusters, as has been previously observed for clusters in this size range $[48,49,50,46]$. Although there was no odd-even theme, size sensitive variations were also observed in the total simulation time required for convergence, as discussed in the following section. 


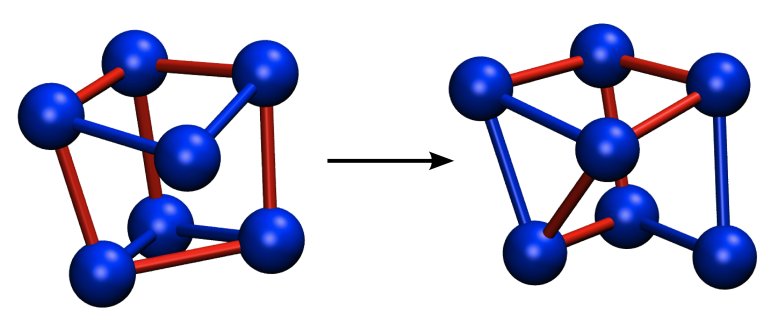

(a) $\mathrm{Ga}_{7}$

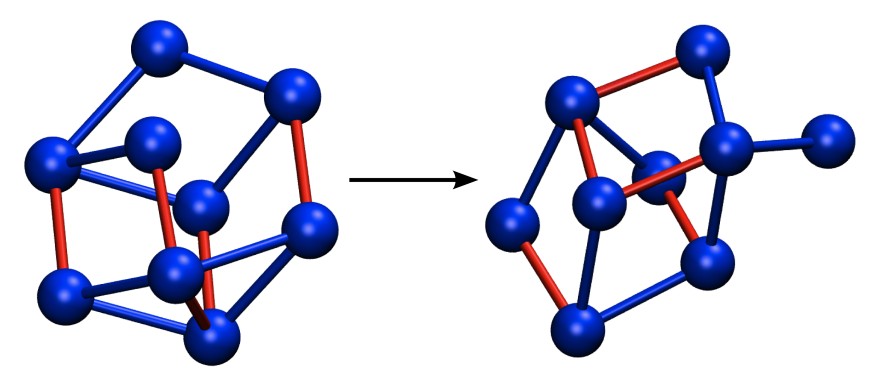

(b) $\mathrm{Ga}_{9}$

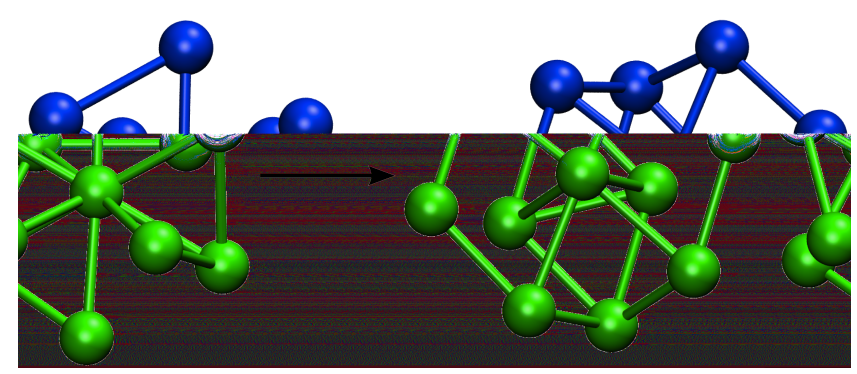

(c) $\mathrm{Ga}_{11}$

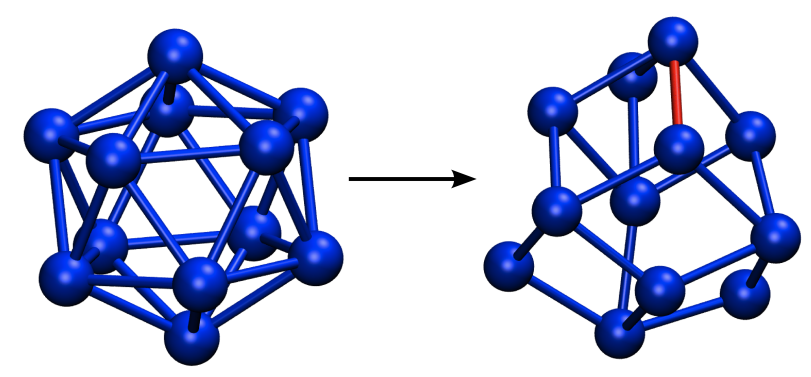

(d) $\mathrm{Ga}_{12}$

Figure 6.2. PT-derived lowest energy structures (right of the arrow) for each cluster size showing a notable structural change from its initial geometry (left of the arrow), where $r_{12}<2.6 \AA$ are shown in red and $2.6 \AA$ $<r_{12}<2.8 \AA$ in blue. Atomic coordinates are given in Tabs. B.27 through B.30. 


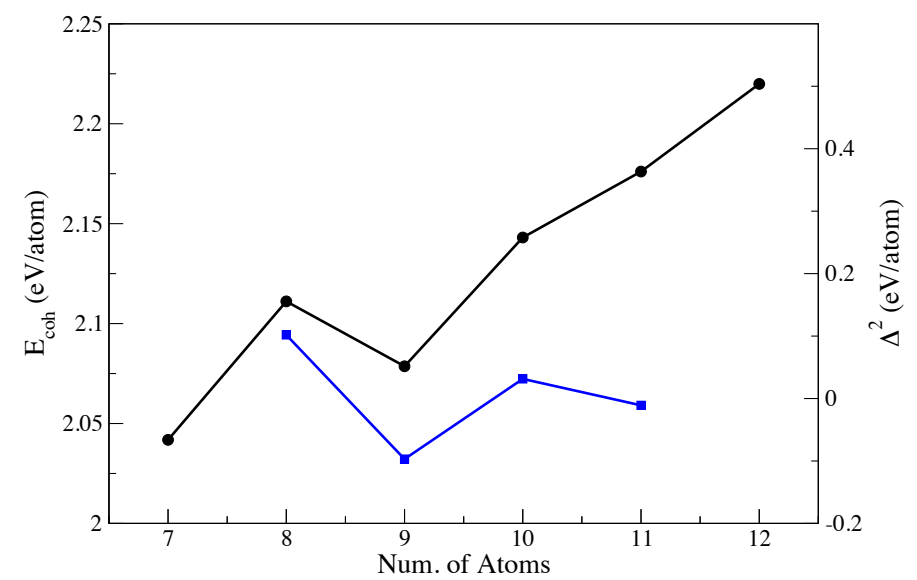

Figure 6.3. Cohesive energies (black) and second order differences (blue) for the PT-derived structures of each microcluster. 


\begin{tabular}{cccccc} 
Cluster & $\mathrm{T}_{\text {peak }}$ & $\mathrm{E}_{\text {peak }}$ & $\boldsymbol{\Delta} \mathrm{T}_{\text {peak }}$ & $\boldsymbol{\Delta} \mathrm{E}_{\text {peak }}$ & Sim. Time \\
\hline $\mathrm{Ga}_{7}$ & 409 & -2.11 & 0.992 & $6.57 \mathrm{e}-4$ & 190 \\
$\mathrm{Ga}_{8}$ & 390 & -2.22 & -0.876 & $-2.04 \mathrm{e}-5$ & 360 \\
$\mathrm{Ga}_{9}$ & 469 & -2.16 & -0.689 & $-4.63 \mathrm{e}-5$ & 220 \\
$\mathrm{Ga}_{10}$ & 517 & -2.23 & -0.108 & $-1.27 \mathrm{e}-5$ & 220 \\
$\mathrm{Ga}_{11}$ & 340 & -2.28 & 0.760 & $1.73 \mathrm{e}-5$ & 240 \\
$\mathrm{Ga}_{12}$ & 178 & -2.36 & -0.073 & $-2.96 \mathrm{e}-6$ & 210
\end{tabular}

Table 6.2. Summary of the key thermodynamic quantities and convergence measures. The units are $\mathrm{K}$ for temperature, $\mathrm{eV} /$ atom for energy, and ps for simulation time. $\Delta \mathrm{T}_{\text {peak }}$ is the change per ps in the temperature of the canonical specific heat curve peak, given in $\mathrm{K} / \mathrm{ps}$, and $\Delta \mathbf{E}_{\text {peak }}$ is the microcanonical analogue in $\mathrm{eV} /$ atom/ps. The total simulation time excludes the initial $10 \mathrm{ps}$ canonical equilibration.

\subsection{Convergence}

The convergence criteria selected for the microclusters was the same qualitative/quantitative measure used for 20-atom cluster, as outlined in Sec. 5.4. The total simulation time required for convergence varied between $190-360 \mathrm{ps}$, depending on the cluster size. The temperature and energy of the peak in the specific heat curves, relative convergence and total simulation time are given in Tab. 6.2. This set of converged specific heat curves for a range of six cluster sizes allowed for thermodynamic analysis to be completed, as discussed in the following section.

\subsection{Thermodynamics}

For each cluster, the canonical and microcanonical multiple histogram specific heat curves are given in Figs. 6.5 and 6.6, respectively. For purposes of comparison, each figure was plotted on a similar vertical scale to those 
of the $\mathrm{Ga}_{20}$ analyses. The relative area under each of the canonical specific heat curve, which would define the latent heat of a phase transition, is generally low making comparisons between the clusters sizes challenging. In comparison to the 20-atom results, each of the microclusters releases significantly less latent heat as the energy (temperature) is raised. The specific heat values generally increase with cluster size, which likely relates to increasing total internal (configurational) energy with cluster size. It is interesting to note, however, that the 8- and 11-atom clusters break this trend, with higher average specific heats than the 9- and 12-atom clusters, respectively. This could be attributed to the symmetric nature of their lowest energy structures (Fig. 6.2). As shown in Fig. 6.3, the 8-atom cluster has a notably high $E_{\text {coh, }}$ which would support a higher specific heat; however, the cohesive energy for the 11-atom cluster is only average.

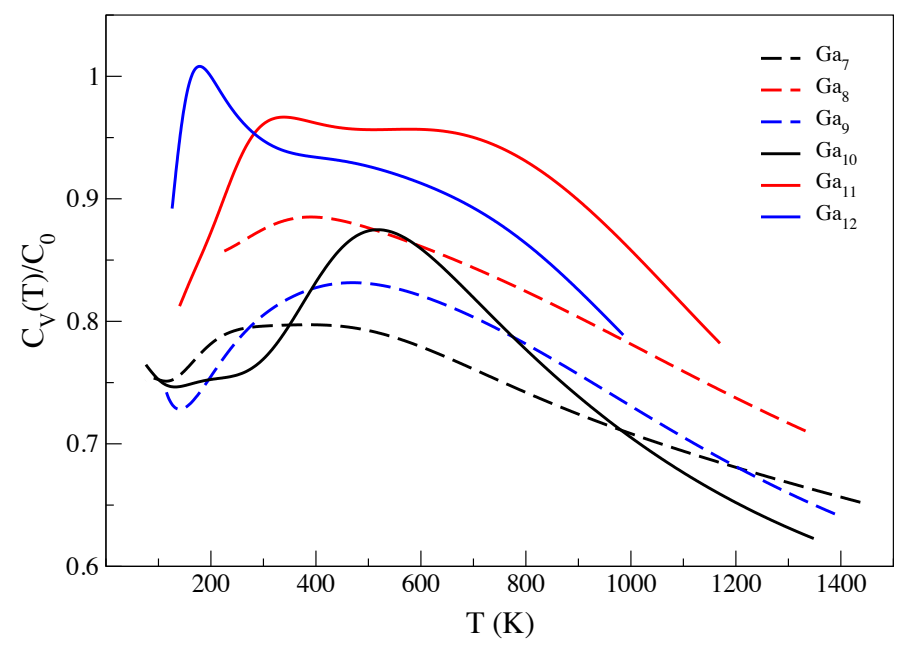

Figure 6.4. Normalized canonical specific heat curves for each of the microclusters.

Only the canonical and microcanonical specific heat curves for $\mathrm{Ga}_{10}$ and $\mathrm{Ga}_{12}$ demonstrate features that could be associated with melting, with a rise and fall to the specific heat surrounding a well-defined peak. This 


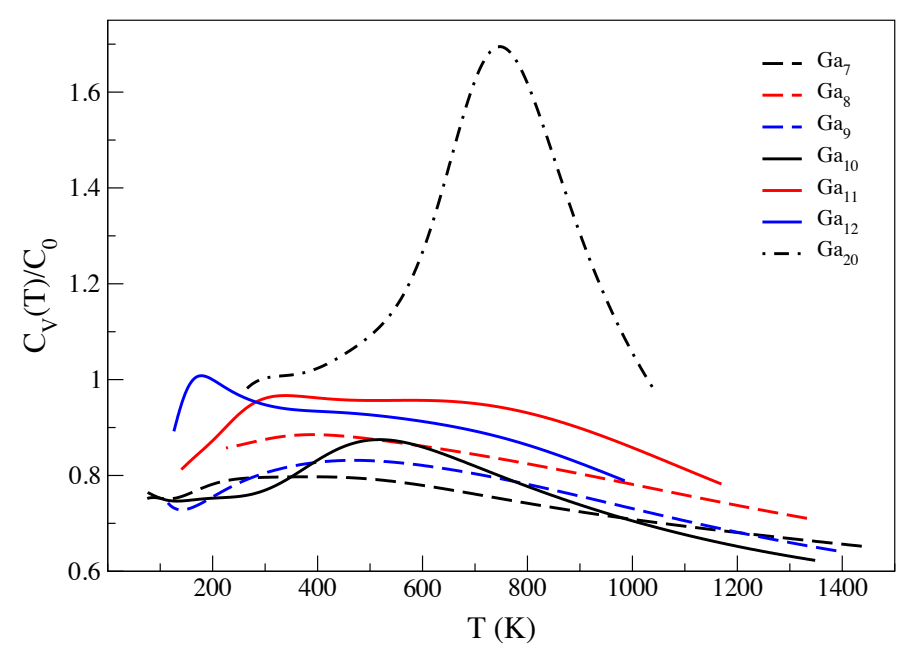

Figure 6.5. Normalized canonical specific heat curves for each of the microclusters with that of the $\mathrm{SC} \mathrm{Ga}_{20}$ cluster, for comparison.

maximum occurs at $590 \mathrm{~K}$ and $-2.30 \mathrm{eV} /$ atom for the 10 -atom cluster and $179 \mathrm{~K}$ and $-2.36 \mathrm{eV} /$ atom for the 12 -atom cluster. It is interesting to note that the temperature associated with the $\mathrm{Ga}_{12}$ specific heat maximum falls well below the bulk melting temperature of gallium. One interesting feature is that the specific heats for both $\mathrm{Ga}_{10}$ and $\mathrm{Ga}_{12}$ drop to lower specific heat values on the high-temperature side of the peak are lower than those on the low-temperature side. This is contrary to what one expects, as liquids generally have a higher specific heat than solids [177, 25]. While the microcanonical curve for the 11-atom simulation also demonstrates a welldefined rise and fall surrounding a specific heat maximum, the canonical simulation lacks this feature. The 7-, 8- and 9-atom microcanonical curves each exhibit a very small, shallow peak, but lack other noteworthy features.

Although some curves exhibit melting-like characteristics, looking only to the specific heat curves leaves an open question as to whether any of the microcluster simulations undergo a solid-liquid phase transition. Adding 


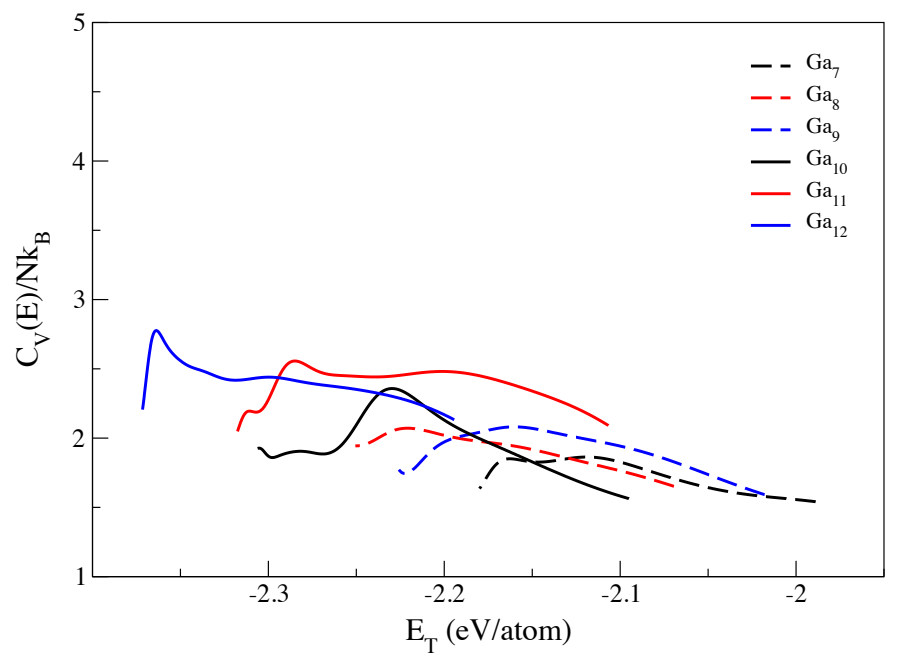

Figure 6.6. The normalized microcanonical specific heat curves for each of the microclusters as a function of total energy per atom.

an analysis of the structural dynamics, as outlined in the following section, offers additional clarity on the possible phase transitions for these tiny systems. 


\subsection{Structural dynamics}

Given the low latent heat associated with the specific heat analysis, the $\delta_{r m s}$ and mean square displacements (MSD) analysis were particularly useful in further exploring the thermodynamics of these microclusters. As annotated in Sections 4.5.2 and 4.5.3, caution should be taken with the exact value of each measure in the region surrounding the phase transition.

The results of the root-mean square analysis are presented in Figs. 6.7 and 6.8 for the average temperature and total energies, respectively. $\mathrm{Ga}_{9}$, $\mathrm{Ga}_{10}, \mathrm{Ga}_{11}$ and $\mathrm{Ga}_{12}$ each exhibit a well-defined melting signature, with low energy $\delta_{\text {rms }}$ values at just above zero followed by a significant rise to values at just below 0.3 . For each of these larger clusters, the temperature and energy corresponding to the maximum specific heat (Figs. 6.5 and 6.6) is annotated by the vertical dashed line. The changes in $\delta_{r m s}$ above these maximum values become smaller, with a smoother increasing progression. As previously noted for the 20-atom cluster, this smoothing of $\delta_{r m s}$ with increasing energy is characteristic of liquid-like behavior.

The $\delta_{r m s}$ values for both the 7 - and 8-atom clusters are notably low across the range of energies. While the bond length variance for the 8-atom cluster shows no signs of melting, the $\delta_{r m s}$ signature for $\mathrm{Ga}_{7}$ is similar to those observed for the larger clusters. The 7-atom cluster shows a low $\delta_{r m s}$ at the lowest energy followed by a steep rise to a smooth progression across the remaining energies; however, it converges to a value of only $\sim 0.2$, while the larger clusters converge to $\sim 0.3$. By visual inspection of movies from each MD simulation, it was confirmed that the 8-atom clusters vibrate with an amplitude that increases with energy, but no atomic swapping or significant structural deformation occurs throughout the simulation, even at the highest energies. MD-generated movies revealed that the 7-atom cluster is only vibrating at the lowest energy, corresponding to the low $\delta_{r m s}$, while all higher energies exhibit atomic position swapping and notable structural deformations. 


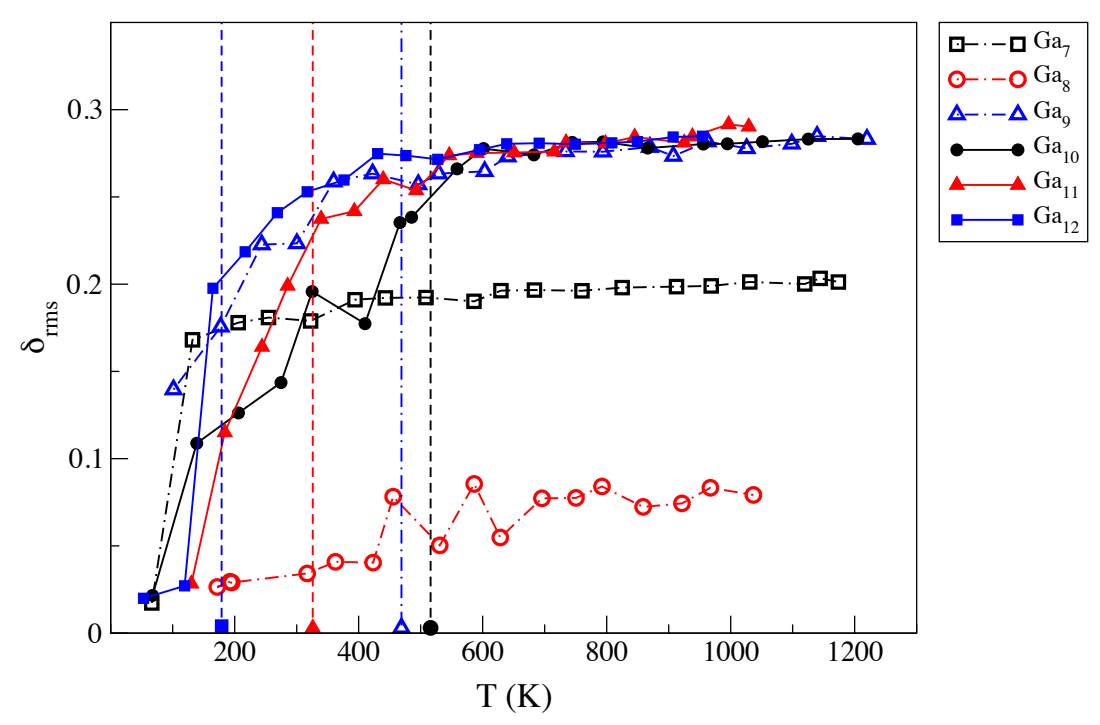

Figure 6.7. Root-mean square bond length variance versus average temperature for each microcluster, with the location of the peak value in the specific heat curves for the four largest clusters annotated by the vertical lines.

The mean square displacements for each simulation offer additional insight. Fig. 6.9 illustrates the MSD for the 7-atom cluster at the lowest and 4 th lowest energies. At the lowest energy, the motion of at least 5 atoms is coupled throughout the simulation. Although the MSD increases very little across the range of energies, atomic motion becomes entirely independent for the 4th lowest energy and greater. For $\mathrm{Ga}_{8}$, coupled atomic motion was noted for all energies. In Fig. 6.10, demonstrating the MSD for the 8-atom simulation, each line represents the motion of two atoms. While the average MSD increases negligibly across the range of energies, the 8-atom cluster has four atoms with approximately half the MSD of the other four. This lower MSD likely arises from the higher coordination for these four atoms, as each have 4 bonds that are less than $2.8 \AA$ while the other four atoms have only 3, as shown in Fig. 6.2 (b). Fig. 6.11 presents 


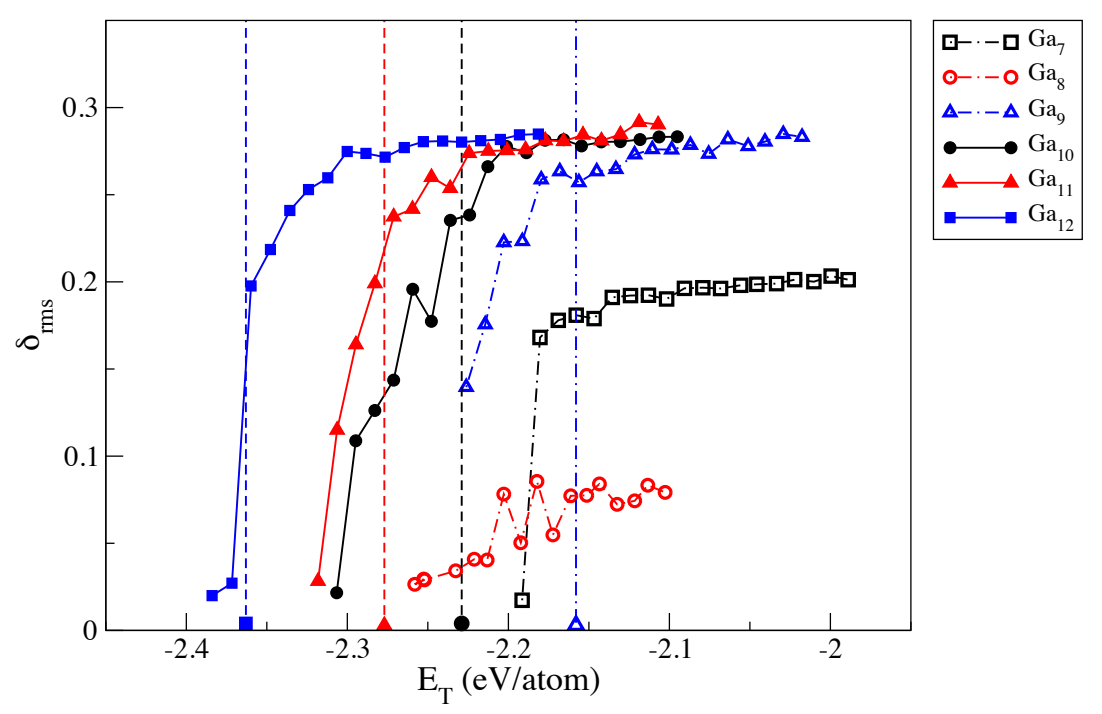

Figure 6.8. Root-mean square bond length variance versus total energy for each microcluster, with the location of the peak value in the specific heat curves for the four largest clusters annotated by the vertical lines.

the MSD for the 9-atom simulation. Similar to the 7-atom MSD, the lowest energy reveals coupled atomic motion for 4 pairs of atoms, while the MSD for the 3rd lowest energy and greater exhibit independent atomic motion.

For the 10-, 11- and 12-atom clusters, the MSD signatures reveal that the central atoms exhibit notably lower atomic mobilities at low energies, as illustrated in Figs. 6.12, 6.13 and 6.14 respectively. In each case, the lowest atomic mobilities could be associated with the highest coordination numbers of the central atoms, as illustrated in Fig. 6.1 and Fig. 6.2. For the 10-atom cluster, the lowest atomic mobilities correspond to the two atoms with a coordination number of 4 at $2.8 \AA$. For the 11- and 12-atom clusters, the single atom with notably lower mobilities correspond to atoms with a coordination number of 6 for the same atomic separation. In each simulation, the highly coordinated central atom becomes equally mobile at higher energies. 


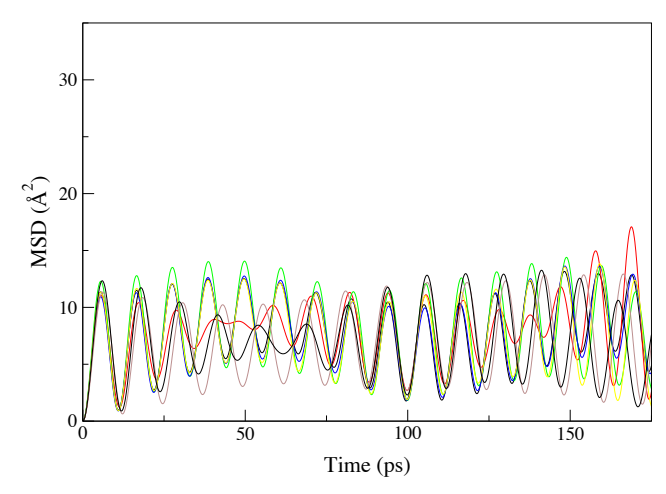

(a)

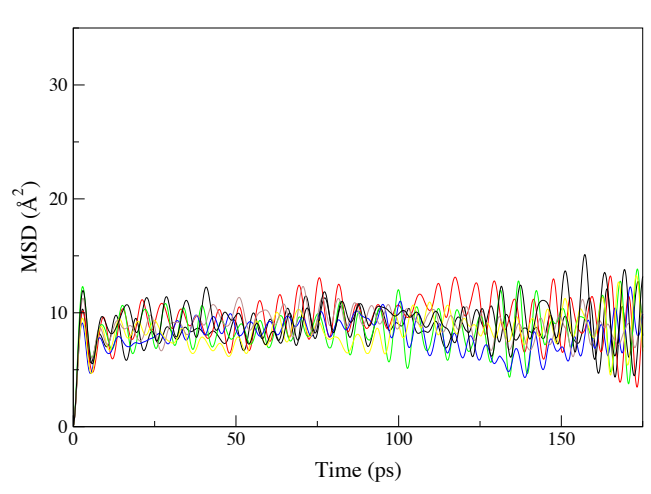

(b)

Figure 6.9. The MSD for $\mathrm{Ga}_{7}$ at the (a) lowest energy and (b) 4th lowest energy. Although the overall MSD increases very little across the range of subrun energies, the lowest energy shows atoms with coupled modes of vibration while 4 th lowest energy (representative of higher energies) shows independent motion for each atom.

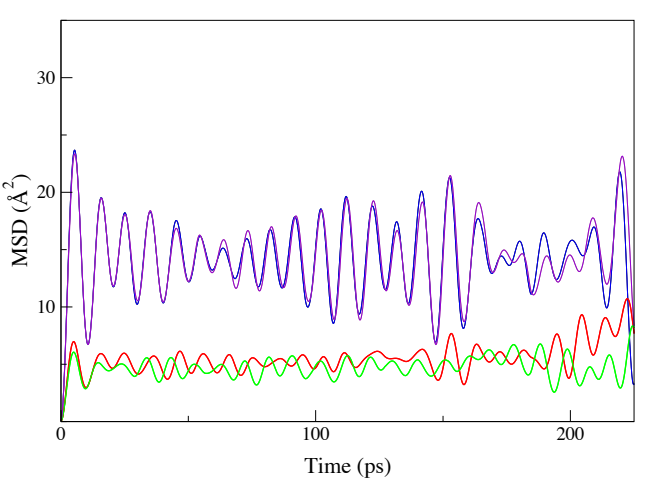

(a)

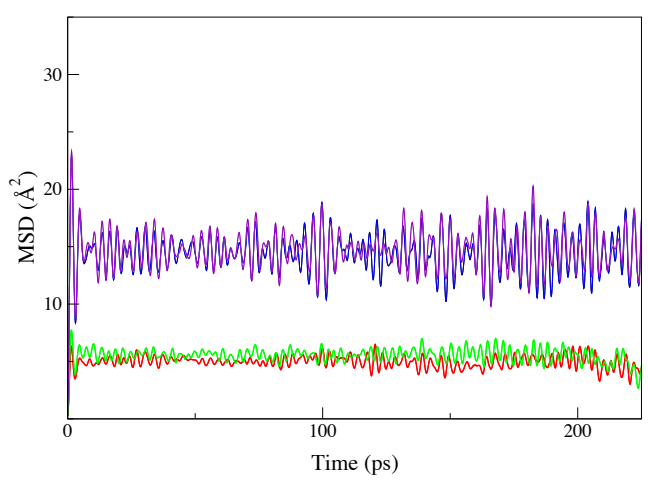

(b)

Figure 6.10. The MSD for $\mathrm{Ga}_{8}$ at (a) the lowest energy and (b) highest energy. The red and green curves (lower mobility) represent the corner atoms with a coordination number of 4 while the blue and purple atoms have a coordination number of 3 , at $2.8 \AA$ as shown in Fig. 6.1. 


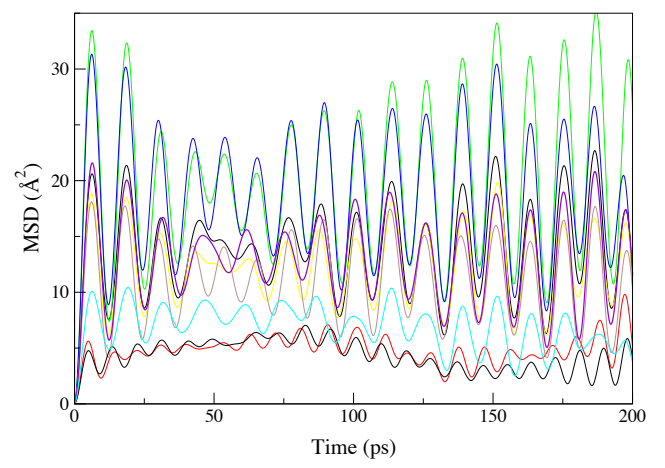

(a)

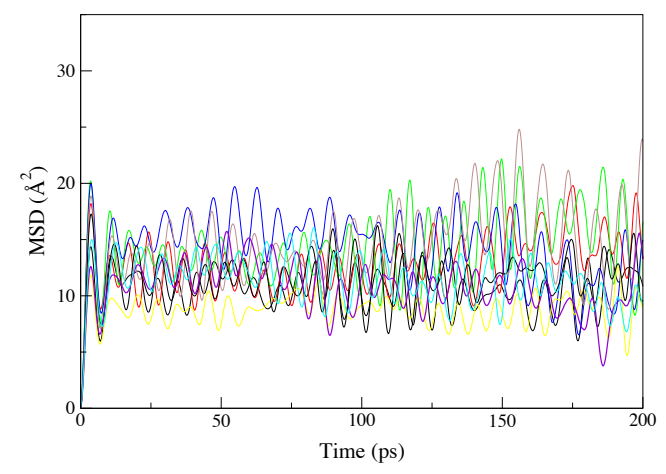

(b)

Figure 6.11. The MSD for $\mathrm{Ga}_{9}$ at the (a) lowest energy, with coupled atomic motion for 4 sets of atomic pairs and (b) 3rd lowest energy, showing independent atomic motion.

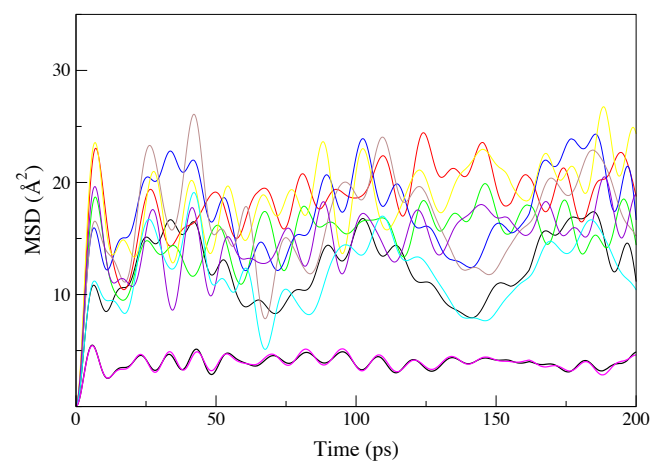

(a)

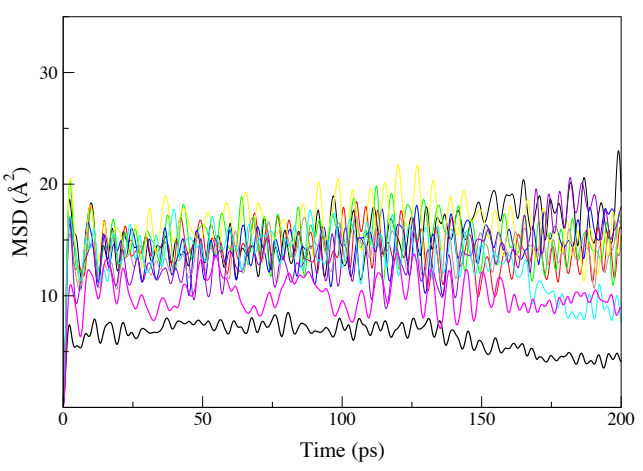

(b)

Figure 6.12. The MSD for $\mathrm{Ga}_{10}$ at the (a) lowest energy, where the atoms with notably lower mobilities represents the two center atoms as shown in Fig. 6.1, and (b) the 8th lowest energy, just below the maximum in the microcanonical specific heat curve, where the two central atoms are becoming increasingly mobile. At higher energies, the two central atoms are equally mobile. 


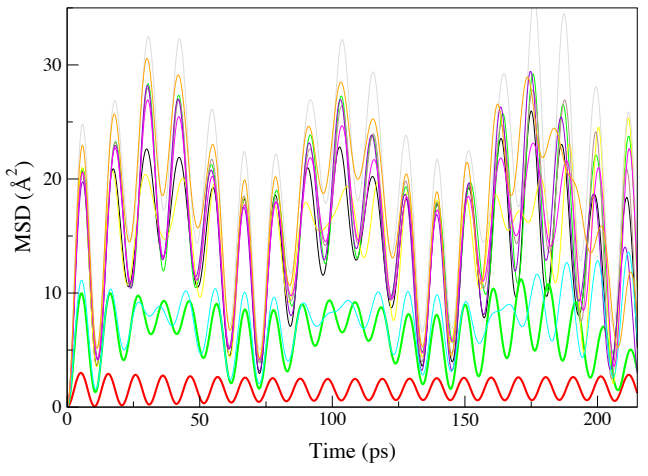

(a)

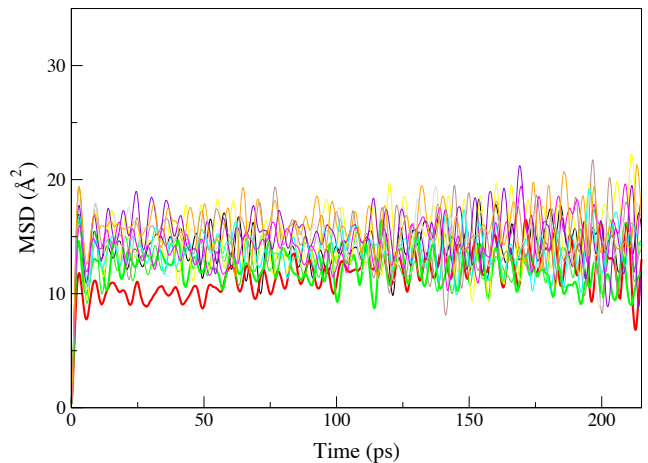

(b)

Figure 6.13. The MSD for $\mathrm{Ga}_{11}$ at the (a) lowest energy, where the red curve with the lowest mobility represents the central atom with a coordination number of 6 at $2.8 \AA$, as shown in Fig. 6.2, and (b) 9th lowest energy just below the peak energy, where the lower mobility of the central atom begins to disappear.

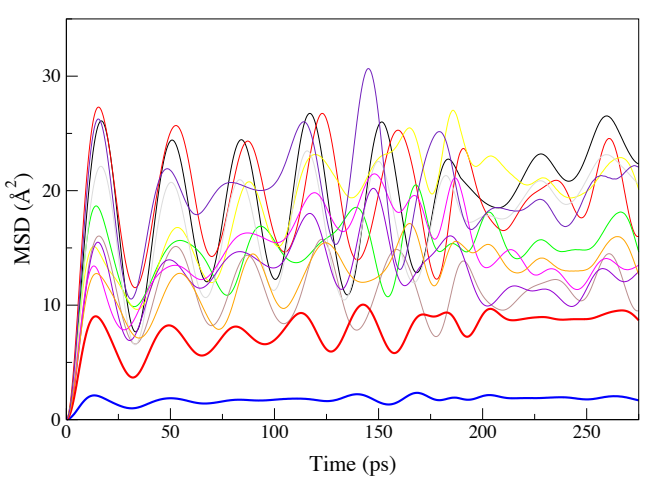

(a)

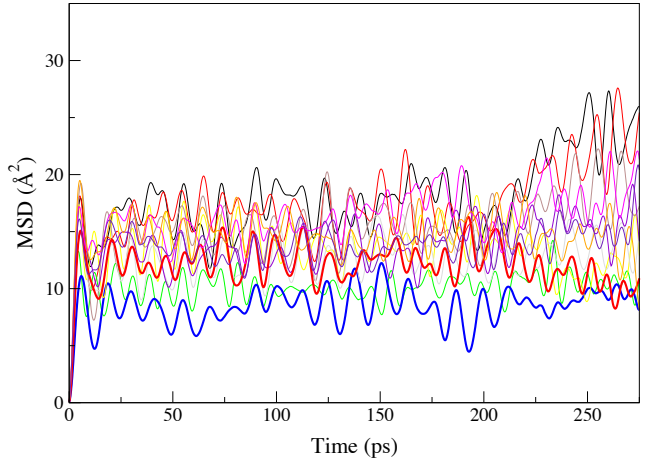

(b)

Figure 6.14. The MSD for $\mathrm{Ga}_{12}$ at the (a) lowest energy and (b) 4th lowest energy. The atom with the lowest mobility represents the central atom on the bottom plane with a coordination number of 6 at $2.8 \AA$, as shown in Fig. 6.2. This lower mobility disappears at higher energies. 


\subsection{Electronic structure}

For each of the microclusters, Bader, electron localization function (ELF) and spherical harmonic-projected electronic density of states (SH-PDOS) analysis were completed for the lowest-energy PT-derived structures. As illustrated in Fig. 6.15, Bader analysis of partial charge revealed that all atoms for the 7- and 8-atom clusters are neutral. The atoms in $\mathrm{Ga}_{9}$ are also predominantly neutral, with the exception of the positively charged ad-atom. For the 10-atom cluster, the two central atoms are negatively charged. The 11- and 12-atom clusters demonstrate charge polarization reminiscent of the 20-atom clusters, with negatively charged central atoms and positive protruding atoms.

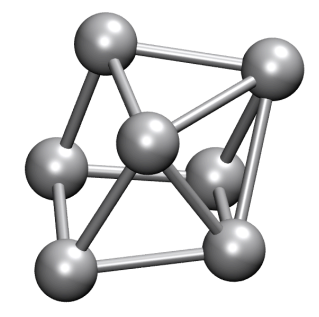

(a) $\mathrm{Ga}_{7}$

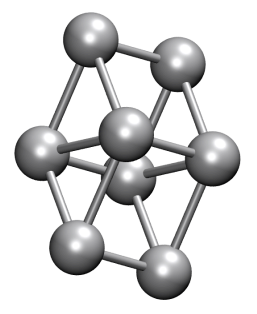

(b) $\mathrm{Ga}_{8}$

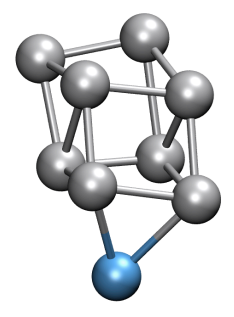

(c) $\mathrm{Ga}_{9}$

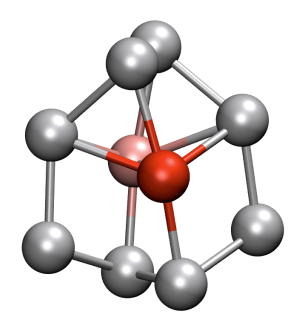

(d) $\mathrm{Ga}_{10}$

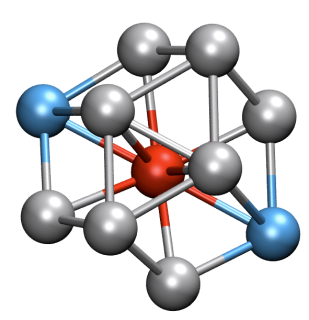

(e) $\mathrm{Ga}_{11}$

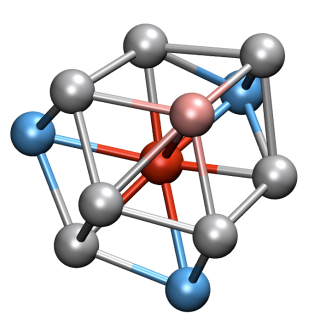

(f) $\mathrm{Ga}_{12}$

Figure 6.15. Bader analysis of partial charge for each of the microclusters: red $=q<-0.2 e^{-} ;$pink $=-0.2 e^{-}<q<-0.1 e^{-} ;$silver $=-0.1 e^{-}<q<0.1 e^{-}$; light blue $=0.1 e^{-}<q<0.2 e^{-}$.

As illustrated in Fig. 6.16, ELF analysis reveals that the electrons for 
each cluster are highly localized around each ionic core. There is no evidence of covalent bonding for any of the microclusters. Surprisingly, this applies to even the 8-atom cluster, although its pair bonding is most similar to that of bulk gallium.

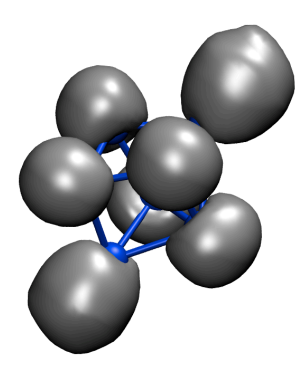

(a) $\mathrm{Ga}_{7}$

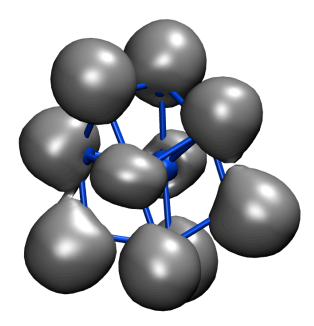

(d) $\mathrm{Ga}_{10}$

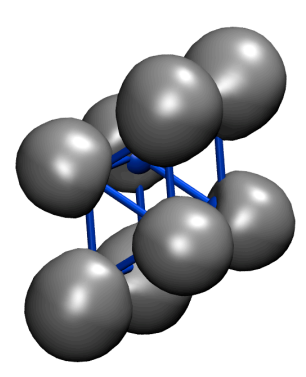

(b) $\mathrm{Ga}_{8}$

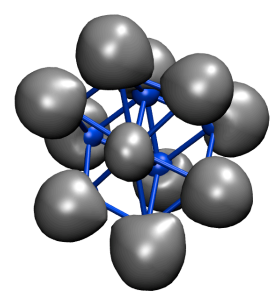

(e) $\mathrm{Ga}_{11}$

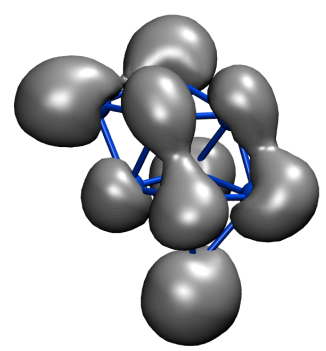

(c) $\mathrm{Ga}_{9}$

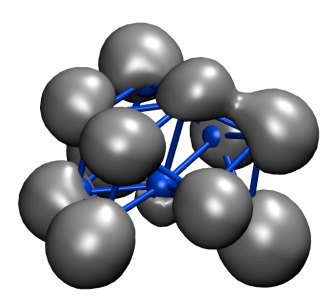

(f) $\mathrm{Ga}_{12}$

Figure 6.16. ELF analysis for each of the PT-derived lowest energy structures at an isovalue of 0.6 .

An analysis of the SH-PDOS was completed for each cluster, given in Figs. 6.17 and 6.18. Each cluster exhibits a slightly metallic nature with a small number of conducting states at the Fermi level. It is interesting to note that the single $1 \mathrm{P}$ state from the 7-atom analysis becomes triplesplit for the 8- and 9-atom clusters, despite the similar rhomboidal motif for each of their lowest energy (PT-derived) structures. This triple-split P state is also a feature of the 10- and 12-atom clusters, although it disappears for the 11-atom cluster. Further analysis reveals that the three separate P-bands arise from $p$-like delocalized shells splitting along the three 
symmetry planes of the rhomboids, most clearly illustrated for the symmetric $\mathrm{Ga}_{8}$ cluster as shown in Fig. 6.19. Although $\mathrm{Ga}_{10}$ does not represent an electronic closed shell with 30 valence electrons, there is a clear change from the 2P-state for the HOMO to 2F-state for the LUMO.

Finally, Figs. 6.20 and 6.21 illustrate the HOMO and LUMO for each of the microclusters. The isovalue for each surface is consistent for all cluster sizes $\left(0.011 e^{-} / \AA^{3}\right)$ and the orientation is maintained between the HOMO and LUMO plot of each cluster, allowing for direct comparison. Both the HOMO and LUMO for the 8-atom cluster have high symmetry, taking on a predominantly $p$ - or $f$-like character. This corresponds well to the SH-PDOS for the 8-atom cluster, given in Fig. 6.17 (b). Although less symmetric, this same nature can be observed for the 7-atom cluster $\mathrm{HOMO} / \mathrm{LUMO}$. It is interesting to note that although the 9-atom structure is very similar to both the 7- and 8-atom, its HOMO and LUMO are quite distinct, taking on no obvious angular momentum character.

The HOMO for the 10-atom cluster is quite symmetric, with a $p$-like character along a central axis parallel to the shorter bonds (shown in red). Although the LUMO for $\mathrm{Ga}_{10}$ lacks symmetry, it resembles the LUMO for the 8-atom cluster, clearly illustrating that the rhomboid structural motif is also evident in the nature of bonding. The HOMO/LUMO for the 11- and 12-atom clusters do not exhibit any clear angular momentum character; however, the high symmetry of the 11-atom cluster is quite evident in both plots, while only the 12-atom LUMO exhibits notable symmetry.

Overall, each of the Bader, ELF, SH-PDOS and HOMO/LUMO analyses revealed similar trends between the cluster sizes, providing no additional data to explain differences in the melting behavior. It is interesting to note that Bader partial charges for the 10-, 11- and 12-atom clusters revealed marked similarities to those of the 20-atom cluster. Although the distinction is not clear, it is also these cluster sizes that reveal specific heat and $\delta_{r m s}$ behavior most indicative of possible phase-transitions. 


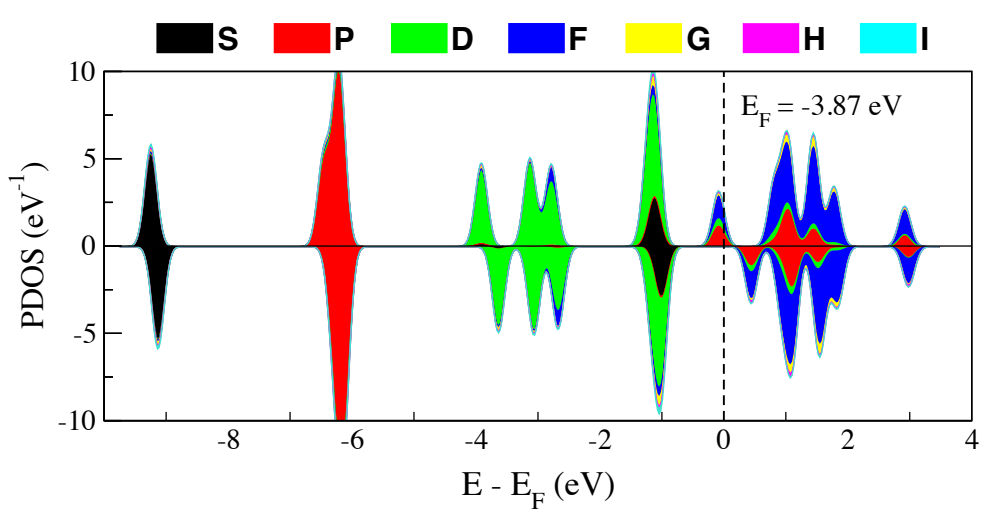

(a) $\mathrm{Ga}_{7}$

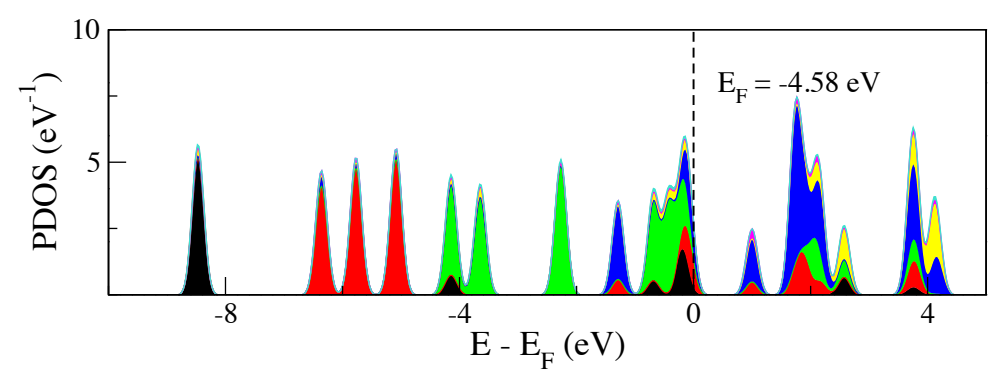

(b) $\mathrm{Ga}_{8}$

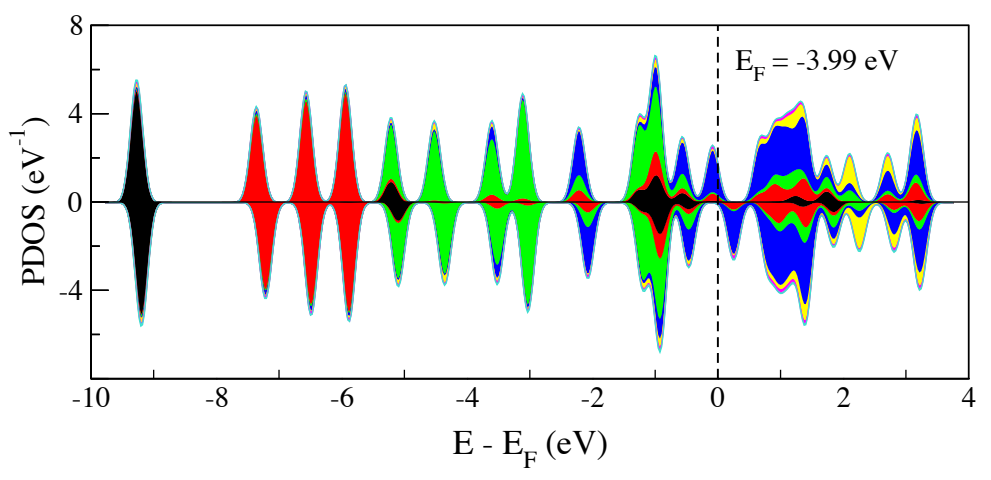

(c) $\mathrm{Ga}_{9}$

Figure 6.17. SH-PDOS for the 7-, 8- and 9-atom PT-derived lowest energy structures. 


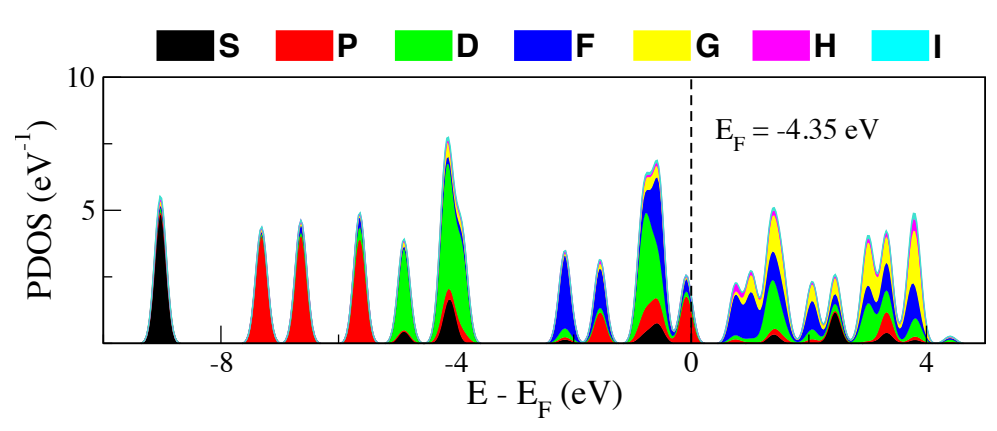

(a) $\mathrm{Ga}_{10}$

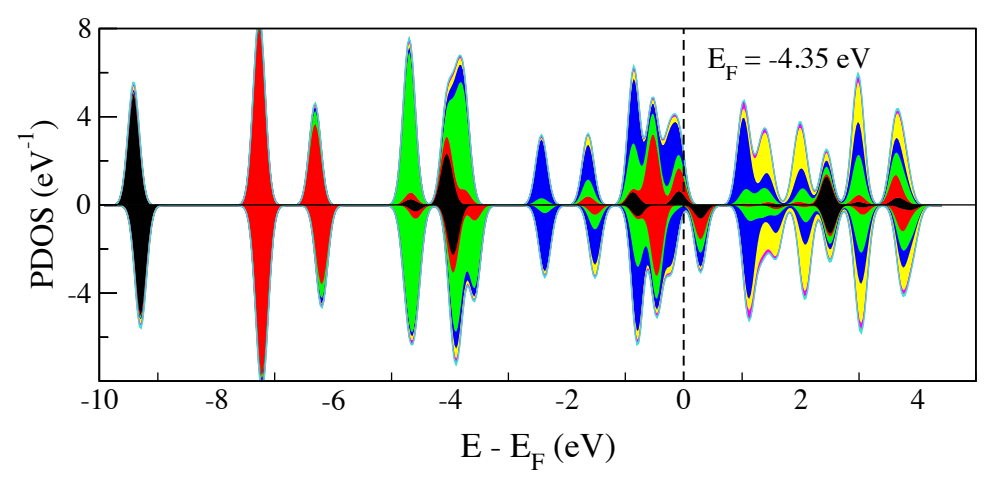

(b) $\mathrm{Ga}_{11}$

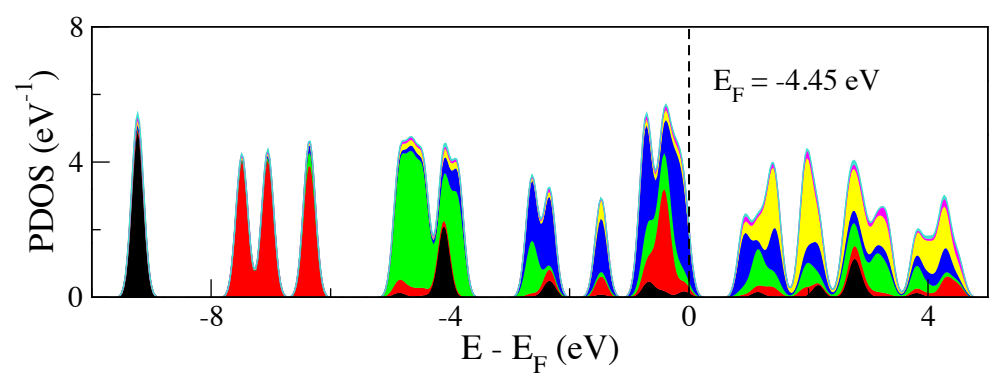

(c) $\mathrm{Ga}_{12}$

Figure 6.18. SH-PDOS for the 10-, 11- and 12-atom PT-derived lowest energy structures 

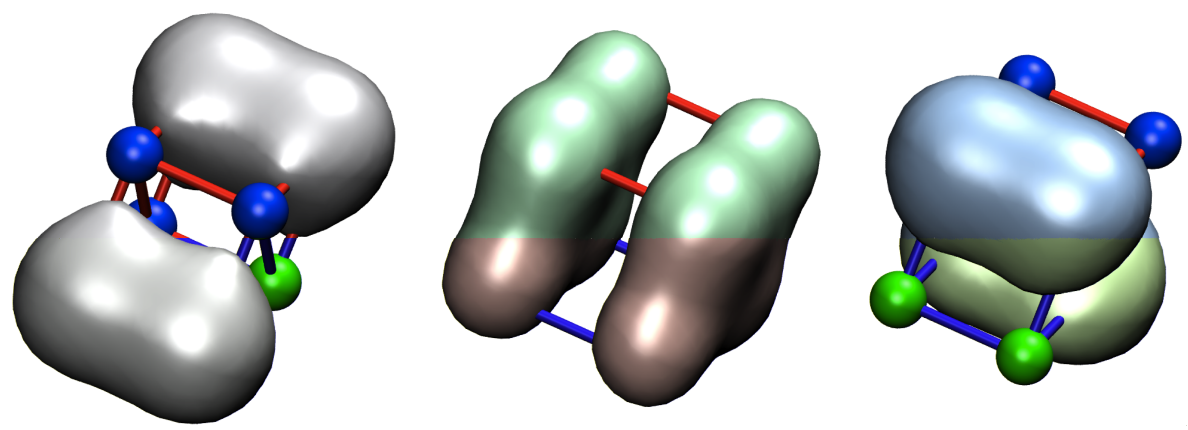

Figure 6.19. The triple-split P-bands for the 8-atom cluster, from left to right in order of highest-to-lowest energy. The charge density isosurface is set to $0.035 e^{-} / \AA^{3}$ 

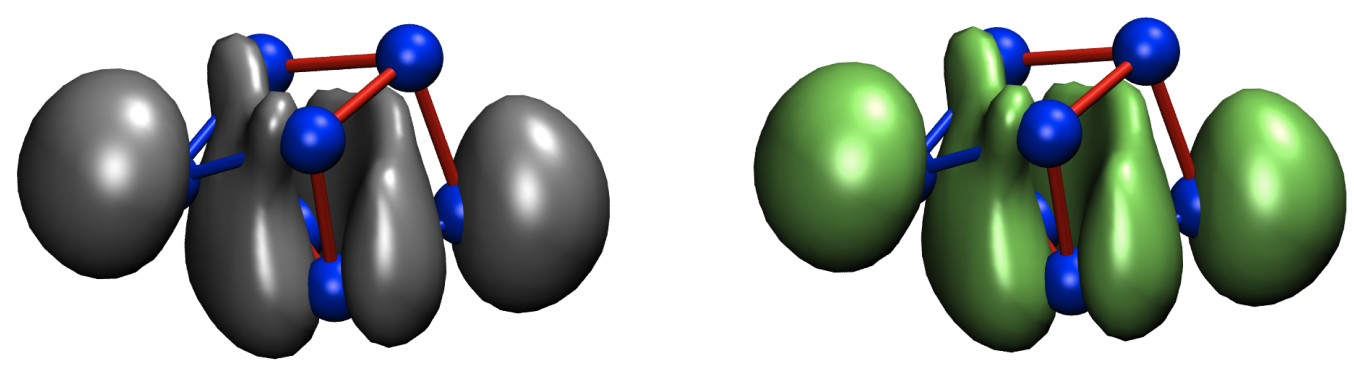

(a) $\mathrm{Ga}_{7}$
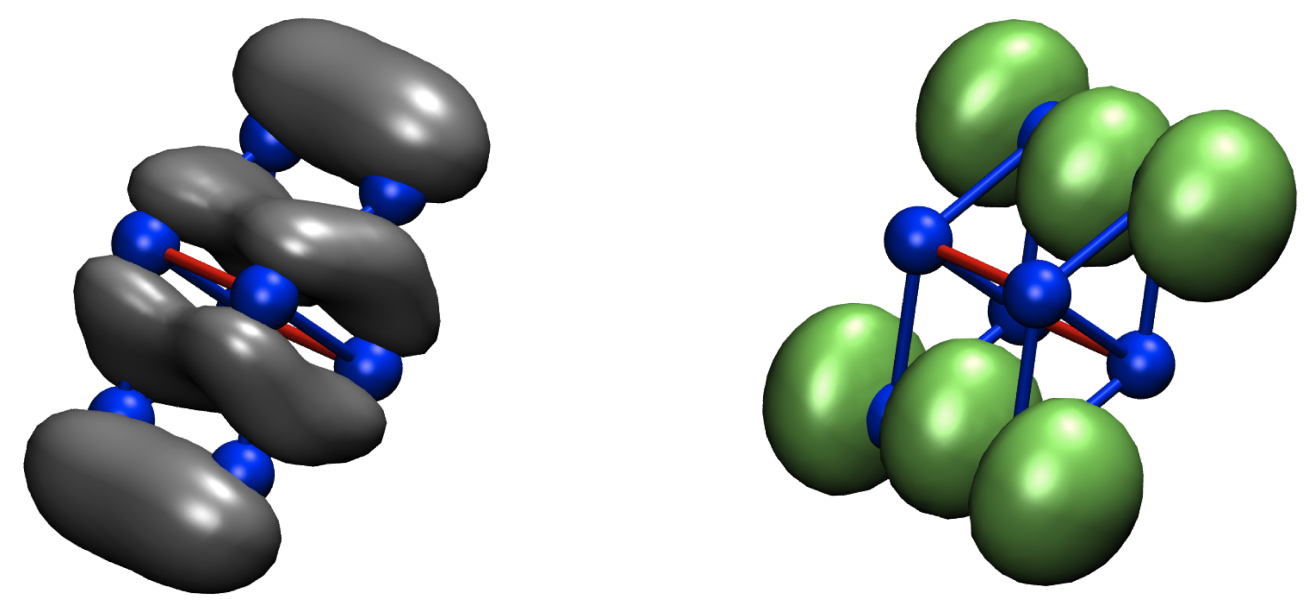

(b) $\mathrm{Ga}_{8}$
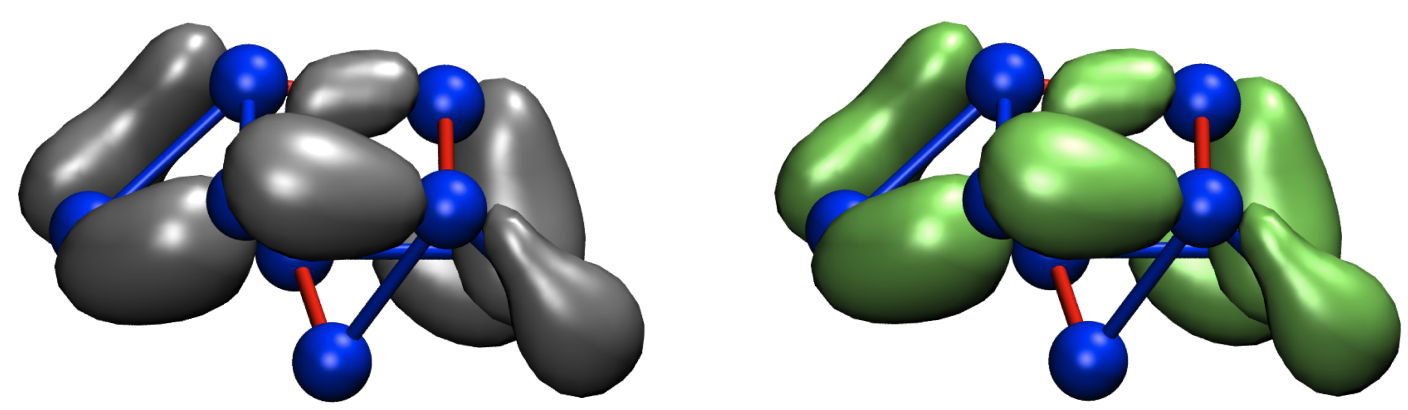

(c) $\mathrm{Ga}_{9}$

Figure 6.20. HOMO (grey) and LUMO (green) for the lowest energy PTderived structures of (a) $\mathrm{Ga}_{7}$, (b) $\mathrm{Ga}_{8}$ and (c) $\mathrm{Ga}_{9}$. The charge density isosurface is set to $0.011 e^{-} / \AA^{3}$. In order to distinguish bond lengths, bonds with $r_{12}<2.6 \AA$ are shown in red and $2.6 \AA<r_{12}<2.8 \AA$ are in blue. 

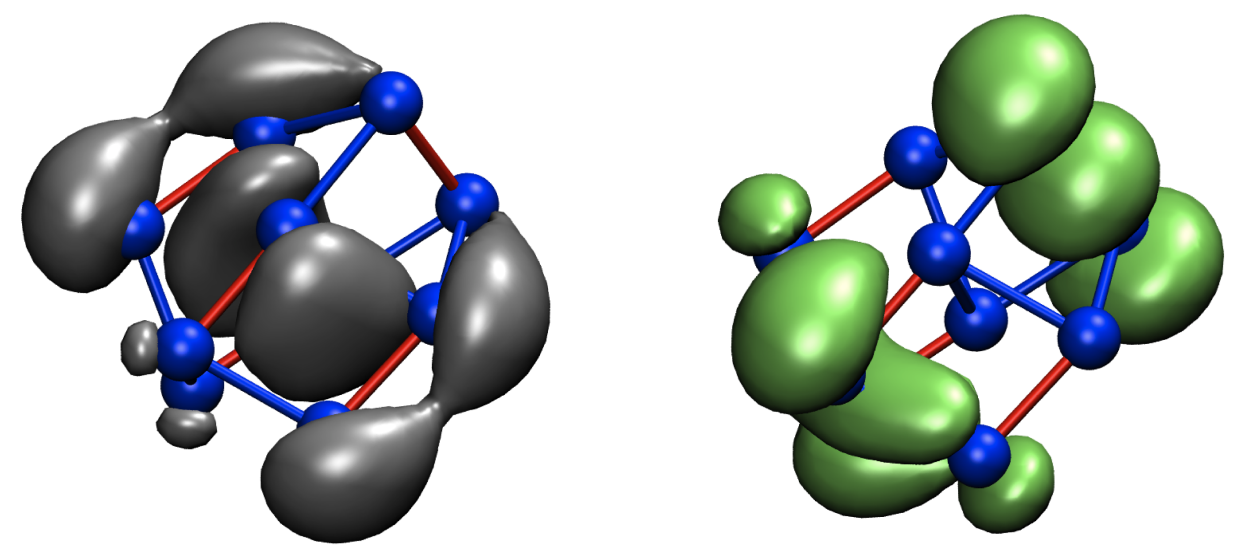

(a) $\mathrm{Ga}_{10}$
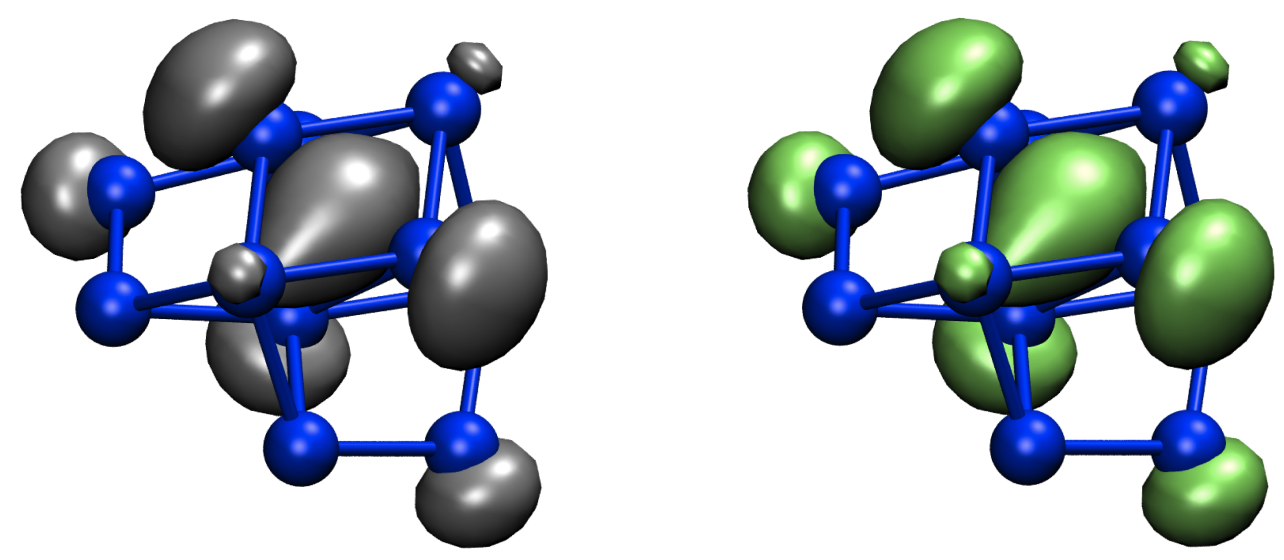

(b) $\mathrm{Ga}_{11}$
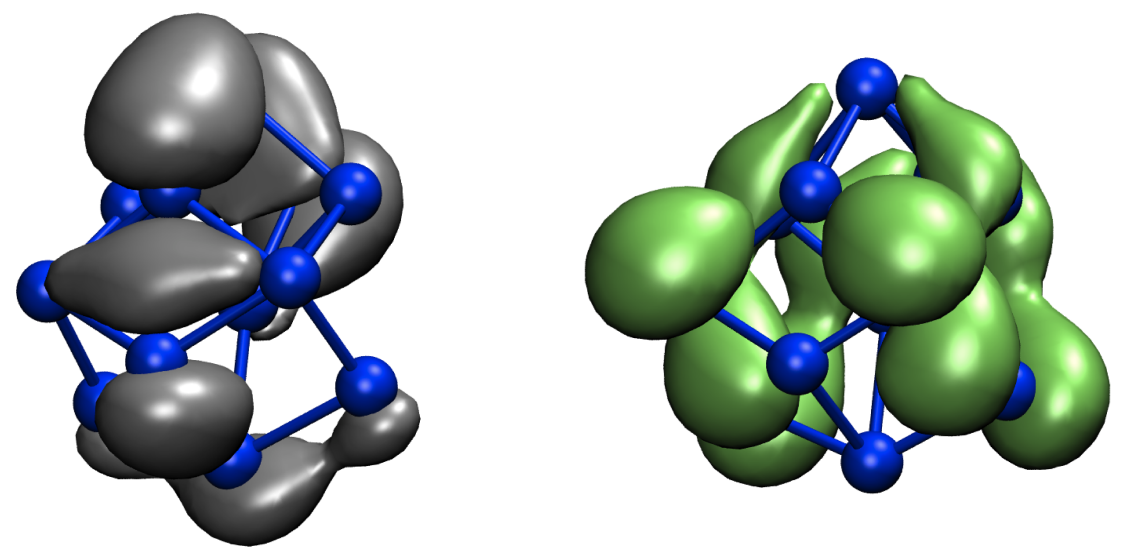

(c) $\mathrm{Ga}_{12}$

Figure 6.21. HOMO (grey) and LUMO (green) for the lowest energy PTderived structures of (a) $\mathrm{Ga}_{10}$, (b) $\mathrm{Ga}_{11}$ and (c) $\mathrm{Ga}_{12}$. The charge density isosurface is set to $0.011 e^{-/ \AA^{3}}$. In order to distinguish bond lengths, bonds with $r_{12}<2.6 \AA$ are shown in red and $2.6 \AA<r_{12}<2.8 \AA$ are in blue. 


\subsection{Summary}

This chapter reports the results of 6 fully converged melting simulations for gallium microclusters sized 7-12 atoms. The lowest energy PT-derived structures for 4 of the 6 clusters show a marked lack of symmetry, while $\mathrm{Ga}_{8}$ and $\mathrm{Ga}_{11}$ are highly symmetric. Each of the PT-derived structures demonstrates a notable rhomboidal motif, although it is significantly distorted for all but the 8-atom cluster.

Thermodynamic analysis revealed low canonical and microcanonical specific heat values for all microclusters. The 10- and 12-atom specific heat curves demonstrate a well-defined specific heat peak. Although a phase transition would be difficult to discern from the specific heat analysis alone, coupling this measure to $\delta_{r m s}$ and MSD analyses yielded additional insight. Given the steep rise of $\delta_{r m s}$ values and smooth convergence above the maximum specific heat temperature/energies for $\mathrm{Ga}_{9}$, $\mathrm{Ga}_{10}, \mathrm{Ga}_{11}$ and $\mathrm{Ga}_{12}$, each of these clusters appears to undergo a phase transition at $469 \mathrm{~K}, 517 \mathrm{~K}, 340 \mathrm{~K}$ and $178 \mathrm{~K}$, respectively. Although the exact values of $\delta_{r m s}$ and MSD in the region surrounding the phase transition due to configuration swaps, the overall nature of each analysis is considered valid across the range. The melting temperatures for the 9- and 10 -atom clusters follow the greater-than-bulk melting temperature trend experimentally observed for larger clusters [25, 30]. The 11-atom cluster exhibits a peak just $37 \mathrm{~K}$ above the bulk melting temperature, which could be within the error of DFT as indicated by the results of the 20-atom simulations. The melting temperature for $\mathrm{Ga}_{12}$, however, is significantly lower than that of bulk gallium and represents the first small gallium cluster that breaks the greater-than-bulk melting trend.

MSD analyses for each of these cluster sizes illustrate mobility changes consistent with a solid-liquid phase transition. The MSD for the 9-atom cluster shows coupled atomic motion at the lowest energy, which changes to independent atomic mobility at energies above melting. The MSD for 
the 10-, 11- and 12-atom clusters each reveal highly-coordinated central atom(s) with significantly lower mobility. These central atoms become equally mobile at energies greater than melting.

The specific heat, $\delta_{r m s}$ and MSD analysis for the 8-atom cluster reveal no evidence of a phase transition. Although the $\delta_{r m s}$ and MSD analysis for the 7-atom cluster show signatures of melting, the low, featureless specific heats and low maximum $\delta_{r m s}$ values make this conclusion questionable. Although visual inspection revealed atomic swapping and notable structural deformation for the 7-atom cluster, the very definition of melting must be questioned for a system with such a small number of atoms.

Bader analysis of partial charge demonstrates that the lowest energy PT-derived structures $\mathrm{Ga}_{10}, \mathrm{Ga}_{11}$ and $\mathrm{Ga}_{12}$ exhibit charge polarization, with negative central atoms and positive protruding atoms. What role this polarization may play in the thermodynamic differences between each cluster size remains unclear. ELF revealed ion-centered electron localization and no evidence of covalent bonding for any of the microclusters. The $\mathrm{SH}-$ PDOS shows a triple-split $1 \mathrm{P}$ shell in the 8-, 9-, 10- and 12-atom clusters arising from $\mathrm{p}$-like delocalized orbitals split along the rhomboidal symmetry planes. Although there were no significant differences in electronic structure that could account for the changes in thermodynamic behavior between cluster sizes, it is interesting to note that the charge polarization and ELF analysis are both remnant of the results for $\mathrm{Ga}_{20}$.

Overall, these results illustrate that melting transitions can be identified using FPMD for gallium microclusters with as few as 9-atoms. For gallium clusters with fewer than 9-atoms, however, melting becomes an ill-defined concept, setting a lower size limit on the very definition of melting. Additionally, the greater-than-bulk melting trend observed for all experimentally measured gallium cluster sizes does not universally apply to all small gallium clusters. In breaking both the definition of melting and greater-than-bulk melting trend, this research establishes important limitations as well as introduces intriguing new questions regarding small 
cluster thermodynamics.

Although these small cluster simulations proved instructive, the fact that the results are unsubstantiated by experiment remains a significant limitation. In order to compare with experiment over an extended size range, a set of melting simulations were also completed for larger gallium clusters sized 32-35 atoms. An overview of these simulations and results are provided in the following chapter. 


\section{Chapter 7}

\section{Large Gallium Clusters}

Having tested our model with the 20-atom cluster and extended our understanding of melting with the small clusters, this chapter investigates small gallium cluster melting over an experimentally measured size range. Specific heat measurements for small gallium cluster cations covered 24 cluster sizes, ranging from 17 to 55 atoms [30, 25, 31, 32]. However, only the sizes 30 through 50 atoms were measured in one-atom increments. These measurements clearly illustrated the one-atom size differences between cluster melting temperatures and latent heat behavior. As a previous study has addressed FPMD melting for the 30- and 31-atom clusters [47], we selected gallium clusters sized 32-35 atoms in order to extend our research to a size range that could be compared to experimental data. The following sections provide an overview of these large gallium cluster simulations.

\subsection{Structures}

Initial seed structures for the large cationic simulations were derived from a set of global minimum (GM) aluminum structures [129]. These structures were each optimized as described in Sec. 5.1, using the LC pseudopotential. As illustrated in Fig. 7.1, the initial structures for $\mathrm{Ga}_{32}^{+}, \mathrm{Ga}_{34}^{+}$ 
and $\mathrm{Ga}_{35}^{+}$show some degree of symmetry while the $\mathrm{Ga}_{33}^{+}$exhibits no symmetric features.

Following these optimizations, microcanonical FPMD parallel tempering simulations were completed for each cation according to the settings referenced in Sec. 5.1, again utilizing the LC pseudopotential model. In order to gauge the accuracy of the model over a size range and address the issue of size-sensitivity, the results were compared to experimentally measured specific heat curves as well as one another. The following section gives the results of the thermodynamic analysis, outlining the comparisons to experimental data and between cluster sizes.

\subsection{Specific Heat Comparisons}

Fig. 7.2 illustrates the simulated canonical specific heat curves compared with experimental data. Overall, $\mathrm{Ga}_{33}^{+}$demonstrates the best agreement with experiment, although remnant of the 20-atom results, the simulated curve has $\sim 0.1$ vertical shift with a melting temperature shift of $\sim 80 \mathrm{~K}$. Unlike the results for $\mathrm{LC} \mathrm{Ga}_{20}^{+}$, however, this shift is to a higher melting temperature as opposed to a lower one. 

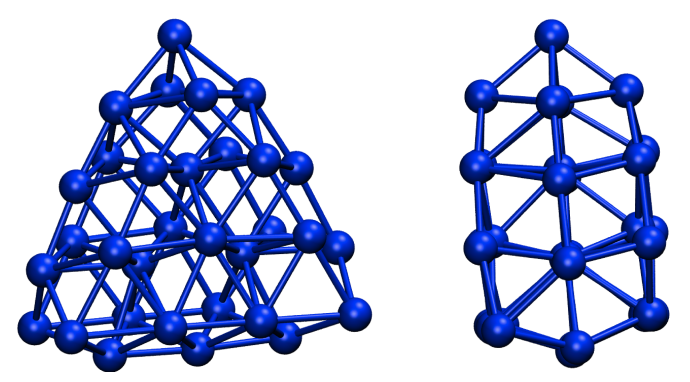

(a) $\mathrm{Ga}_{32}^{+}$
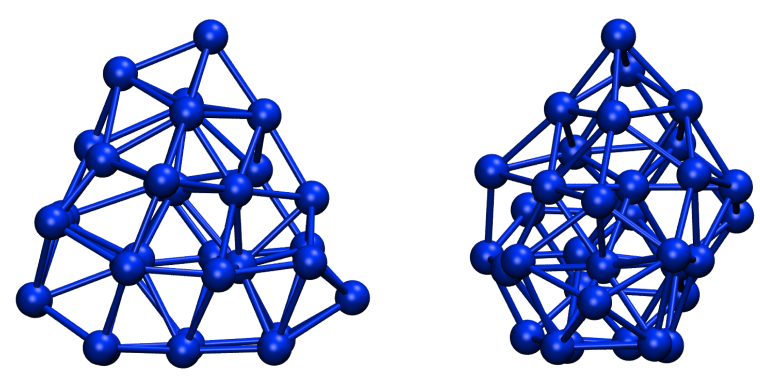

(b) $\mathrm{Ga}_{33}^{+}$
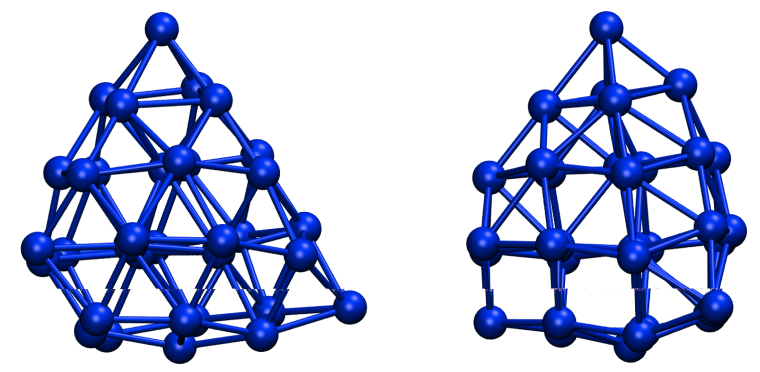

(c) $\mathrm{Ga}_{34}^{+}$
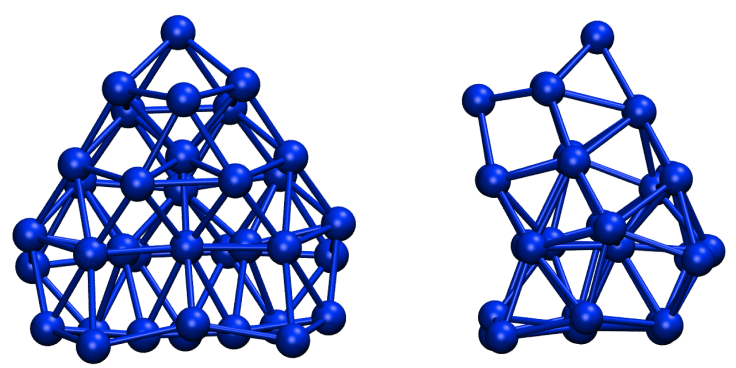

(d) $\mathrm{Ga}_{35}^{+}$

Figure 7.1. Two views for the 32-,33-, 34- and 35-atom gallium cationic initial structures, derived from GM aluminum clusters [129] optimized using the gallium LC PAW pseudopotential as outlined in Sec. 5.1. Atomic coordinates are given in Tabs. B.31 through B.34. 


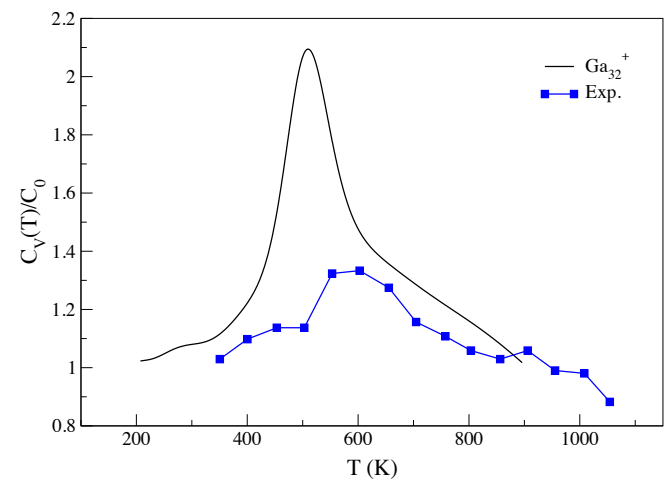

(a)

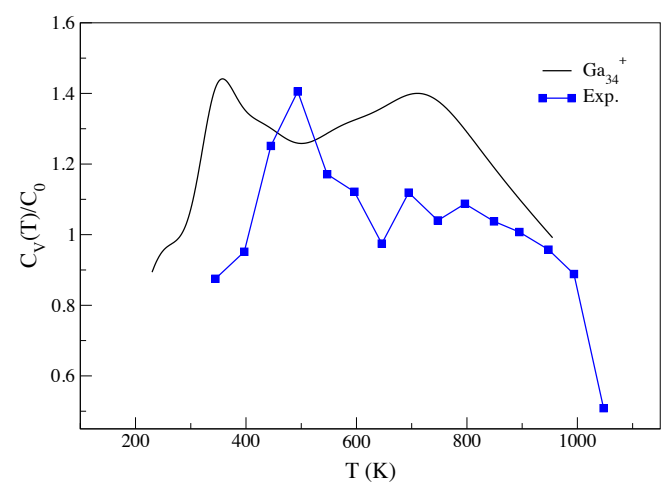

(c)

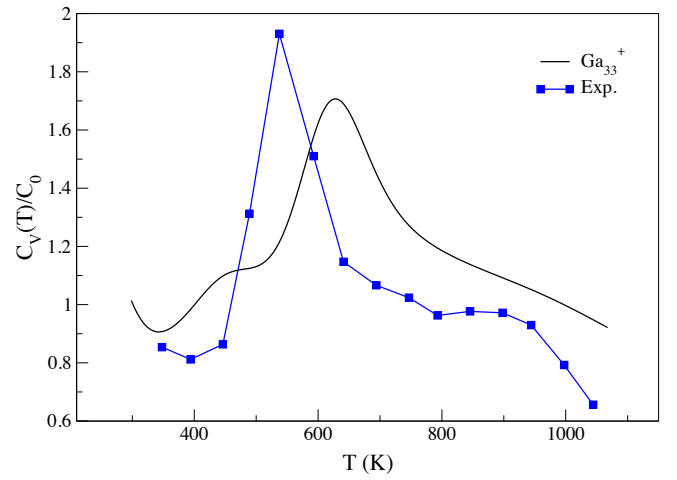

(b)

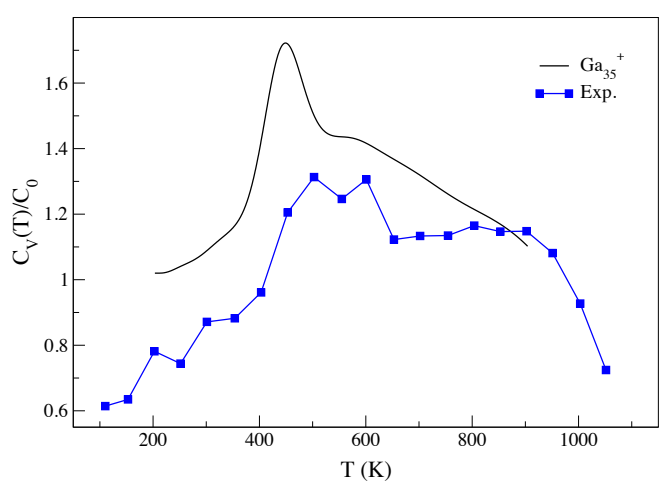

(d)

Figure 7.2. Simulated and experimental canonical specific heat curves for (a) $\mathrm{Ga}_{32}^{+}$, (b) $\mathrm{Ga}_{33}^{+}$, (c) $\mathrm{Ga}_{34}^{+}$and (d) $\mathrm{Ga}_{35}^{+}$. 
The simulated specific heat curve for the 32-atom cation demonstrates a very high, narrow peak, in contrast to the truncated experimental curve. As the experimental measurements are made in approximately $50 \mathrm{~K}$ temperature steps, a measurement error for a single specific could have missed this high, narrow peak. However, given that the experiments measured multiple specific heat curves, it is more likely that the simulation is overestimating the latent heat of melting. The simulated melting temperature is shifted $\sim 75 \mathrm{~K}$ lower than the experimentally measured $T_{m}$ for $\mathrm{Ga}_{32}^{+}$.

Neither the $\mathrm{Ga}_{34}^{+}$or $\mathrm{Ga}_{35}^{+}$results capture the experimental latent heat behavior with any degree of accuracy. Although the low, wide nature of the 34-atom canonical specific heat is generally in good agreement with experiment, the second rise to a peak near $750 \mathrm{~K}$ is entirely distinct. Additionally, both the 34- and 35-atom cluster exhibit melting transitions at temperatures that are $140 \mathrm{~K}$ and $75 \mathrm{~K}$ lower than the corresponding experimental $T_{m}$.

A summary of the modelled and experimental melting temperatures is provided in Fig. 7.3. As can seen from this curve, the general trend of the melting temperatures is matched quite well, with the exception of the 33-atom simulation. Each of the clusters in this size range melts at temperatures exceeding that of bulk gallium.

Figs. 7.4 and 7.5 illustrate the canonical and microcanonical specific heat curves compared among the simulated cluster sizes. $\mathrm{Ga}_{34}^{+}$has a low melting temperature, only $53 \mathrm{~K}$ higher than bulk and significantly lower than the other clusters in this size range. Additionally, the canonical specific heat curve for the 34-atom cluster demonstrates a lower, broader melting transition resembling a "non-melter," compared to the magic melting transitions with a relatively narrow, well-defined peak exhibited by the 32-, 33- and 35-atom cations.

Comparing between canonical and microcanonical curves, the nature of the microcanonical specific heat curves matches that of the canonical specific heats for every simulation except the 34-atom cation. Rather than 


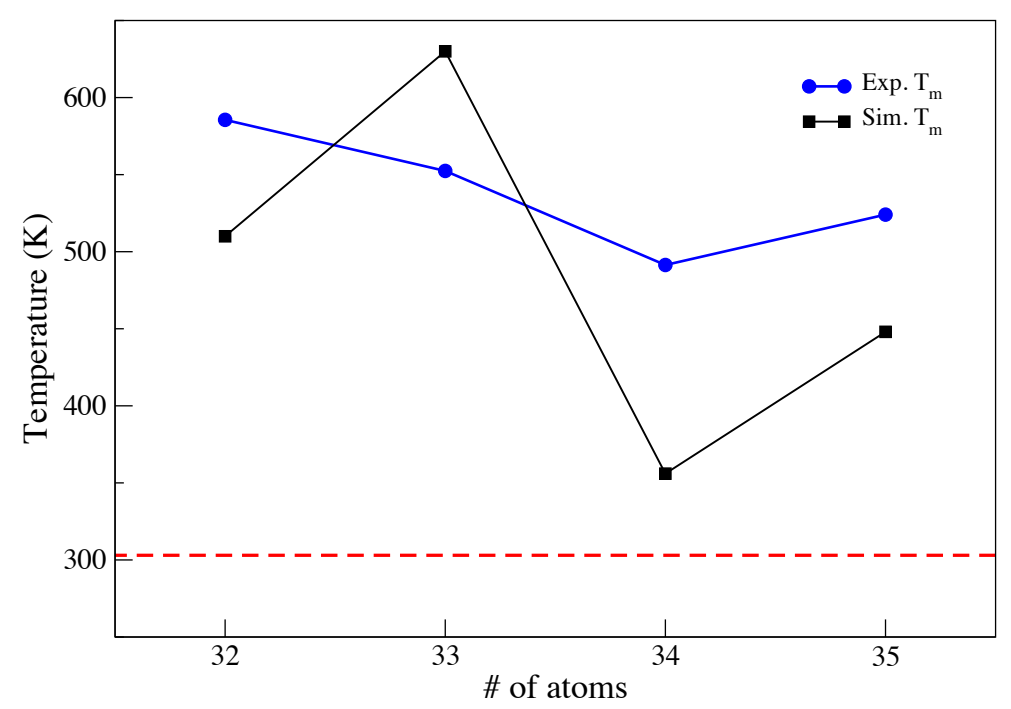

Figure 7.3. A summary of the simulated (black) and experimental (blue) melting temperatures for each of the clusters sized 32-35 atoms, compared with the bulk melting temperature of gallium (red dashed) at $303 \mathrm{~K}$.

showing a wide, broad melting transition, the microcanonical curve shows a small, well-defined peak at low energies followed by higher temperature peaks. Similar peaks are also observed for the $\mathrm{Ga}_{35}^{+}$atom cluster. Analysis of the average radial distribution functions for this simulation reveals that these peaks do not correspond to any significant structural transitions.

In order to further probe the thermodynamic behavior, a root-mean square bond length analysis was completed for each cluster size. As noted in Sec. 4.5.2, the exact values for this measure should be taken with caution in the region surrounding the phase transition. Figs. 7.6 and 7.7 give the $\delta_{r m s}$ results plotted against the average temperature and energy of each simulation subrun. For the 32- and 33-atom simulations, the temperature and energy of the specific heat peaks, shown by the vertical dashed lines, correlates well to the onset of smooth, high values of $\delta_{r m s}$ values.

Although the high-energy values of $\delta_{r m s}$ for $\mathrm{Ga}_{34}^{+}$appear to be wellconverged, the lower energy values are clearly not. In fact, at low ener- 


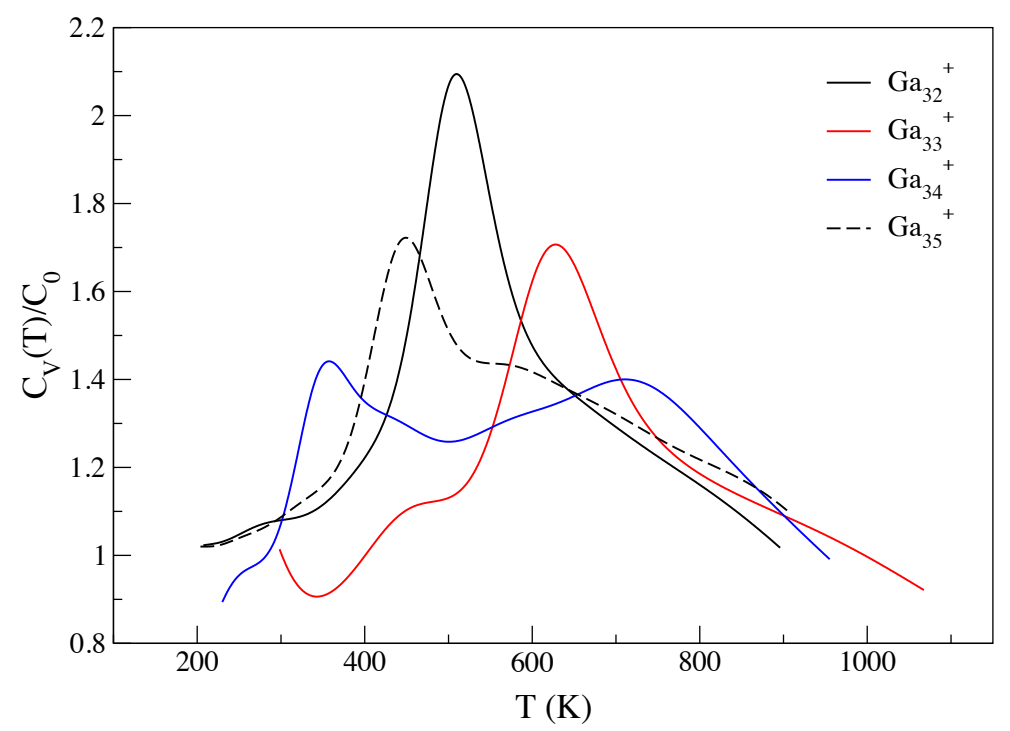

Figure 7.4. The normalized canonical specific heat curves for each of the large cationic clusters.

gies, the root-mean square bond length variance for the 34-atom cluster is relatively high, indicating that the structures at the lowest temperatures are already less rigidly bound than the 32- or 33-atom structures at comparable temperatures. Additionally, the peak in the specific heat curve does not match the onset of the smoother, higher values, again indicative of non-convergence. While there is a steep rise in bond length variance near the melting temperature for the 35-atom cluster, the $\delta_{r m s}$ values appear non-converged at all simulation energies.

Although not shown here, mean square displacements were also computed for each of the clusters in this size range. Similar to the 20-atom and small cluster results, this analysis demonstrated that the central atoms have $1 / 4$ the mobility of the surface atoms. These central atoms become equally mobile to the surface atoms at the melting energy.

From the specific heat, $\delta_{r m s}$ and MSD analyses, it does appear that each cluster undergoes a phase transition. However, the large variation in melt- 


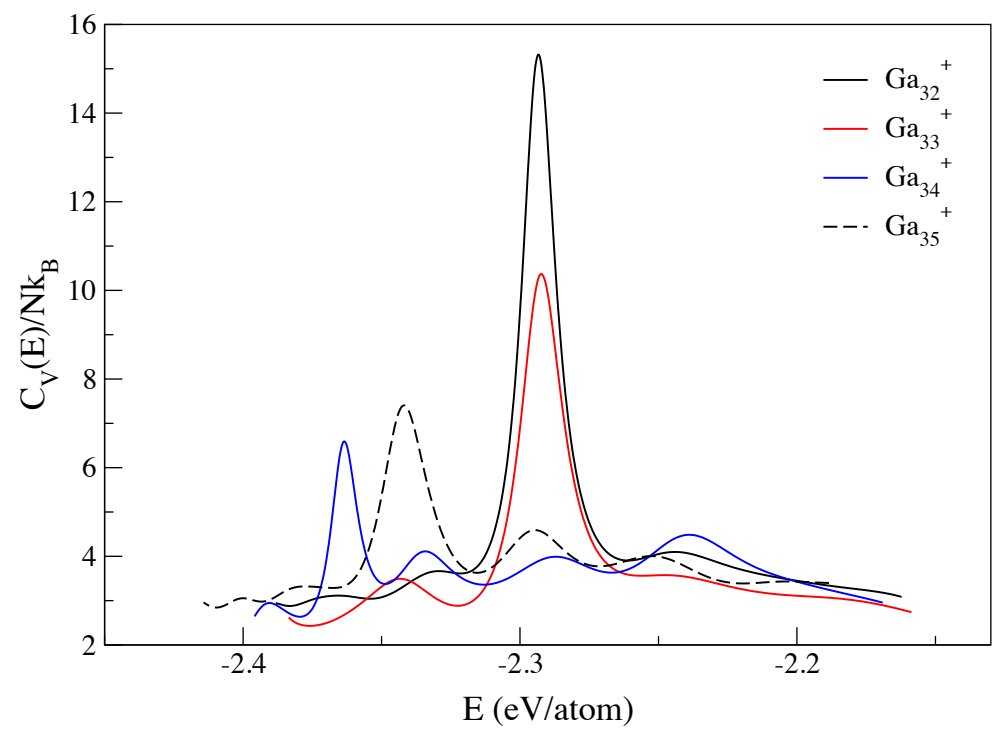

Figure 7.5. The normalized microcanonical specific heat curves for each of the large cationic clusters.

ing temperatures across the size range cannot be explained by trends in the cohesive energies. In order to further probe these thermodynamic variations, an analysis of the lowest energy parallel tempering (PT) derived structures was completed for each simulation. 


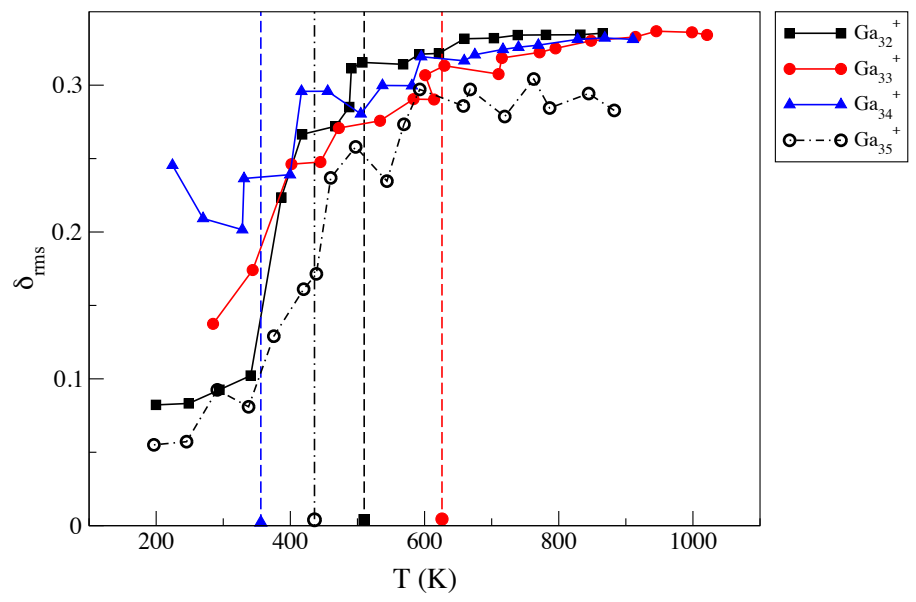

Figure 7.6. Root-mean square bond length variance versus average temperature for each of the large cluster cationic simulations. The location of the peak value in the specific heat curves for each cluster annotated by the vertical lines.

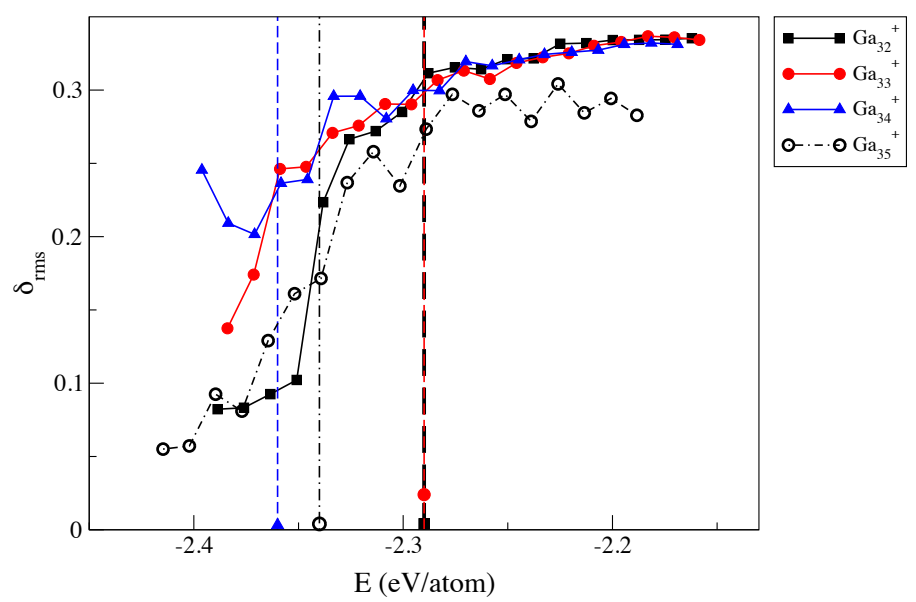

Figure 7.7. Root-mean square bond length variance versus total energy for each of the large cluster cationic simulations. The location of the peak value in the specific heat curves for each cluster annotated by the vertical lines. 


\begin{tabular}{cccc}
\hline \hline Cluster & $\mathbf{E}_{\mathbf{I n i t}}(\mathbf{K})$ & $\mathbf{E}_{\mathbf{P T}}(\mathbf{e V} /$ atom $)$ & $\Delta \mathbf{E}$ \\
\hline $\mathrm{Ga}_{32}^{+}$ & 2.453 & 2.453 & - \\
$\mathrm{Ga}_{33}^{+}$ & 2.441 & 2.473 & 0.032 \\
$\mathrm{Ga}_{34}^{+}$ & 2.450 & 2.457 & 0.007 \\
$\mathrm{Ga}_{35}^{+}$ & 2.463 & 2.463 & - \\
\hline $\mathrm{Ga}_{20}^{+}$ & & 2.366 & \\
\hline
\end{tabular}

Table 7.1. Summary of the cohesive energies for the optimized initial structure $\left(E_{\text {Init }}\right)$ and lowest energy parallel tempering discovered structure $\left(E_{P T}\right)$. Energies are in $\mathrm{eV} /$ atom. The cohesive energy for the 20-atom cation (LC model) is also included for comparison.

\subsection{PT-derived Structures}

Optimizations were completed for the lowest energy PT-derived clusters in accordance with the settings described in Sec. 5.1. The PT-derived structures are identical to the starting geometries for $\mathrm{Ga}_{32}^{+}$and $\mathrm{Ga}_{35}^{+}$simulations, but are entirely distinct for $\mathrm{Ga}_{33}^{+}$and $\mathrm{Ga}_{34}^{+}$simulations. Fig. 7.8 illustrates the structural differences between the 33- and 34-atom initial and PT-derived structures. It is interesting to note that while the 33-atom structure becomes more symmetric, the 34-atom structure becomes considerably less symmetric.

The initial and PT-derived cohesive energies are given in Tab. 7.1, demonstrating that the 33- and 34-atom PT-derived structures have lower cohesive energy than their initial seed structures. As a point of comparison, the cohesive energy for the $\mathrm{LC} \mathrm{Ga}_{20}^{+}$simulations is also included. This energy is significant lower than that of the large clusters, which likely relates to surface vs. bulk effects. All but one atom for $\mathrm{LC} \mathrm{Ga}_{20}^{+}$cappedsphere structure are surface atoms, yielding a 5\% interior-to-surface ratio, whereas these large cationic clusters have between 3 and 4 central atoms, giving them $\sim 10-13 \%$ interior-to-surface ratios. 
Fig. 7.9 demonstrates the relative cohesive energy as a function of cluster size for each of the large cationic clusters, illustrating the persistence of the odd-even trend even at these large cluster sizes. The higher cohesive energies now belong to the odd-sized clusters, correlating to an even number of electrons for each odd-numbered cation. The second-order differences are included for completeness. The limited size range makes an odd-even trend impossible to identify, although it is noted that the two values calculated follow the same trend as the cohesive energy.

For comparison, gallium cationic structures obtained by an extensive GM search [46] were also investigated. For the 32-, 33- and 35-atom clusters, these structures were nearly identical to the PT-derived structures, with only slight bond length differences arising due to the use of a different pseudopotential model in the GM search. For the 34-atom cluster, however, the GM structure was significantly different to both the initial and PT-derived structures. This interesting structure, as shown in Fig. 7.10 , is very similar to that of the lowest energy $\mathrm{Ga}_{33}^{+}$structure, with an adatom on one of the cluster faces. Optimized according to the settings listed utilized in this research (outlined in Sec. 5.1), this structure has higher a cohesive energy ( $2.460 \mathrm{eV} /$ atom) than the lowest energy PT-derived structure. It is also interesting to note that although the initial, PT-derived and GM structures are each quite distinct, their cohesive energies are quite similar.

Looking to trends in the lowest energy structures identified in this size range ( $\mathrm{GM}$ for $\mathrm{Ga}_{34}^{+}$and PT-derived for the other three sizes), a tripleplane symmetry can be observed in all cluster sizes. While these larger cluster sizes are far from bulk-like structures, it is interesting to note that this plane-like structure is quite remnant of the metallic planes observed in bulk gallium. As the dimeric nature of the bulk-like atoms is absent, however, these structures more likely represent the very stable, metallic bi-layers outlined in X-ray diffraction studies of bulk gallium surfaces [15]. The triple-plane would represent two metallic bi-layers sharing the central 
plane. The inter-planar separation for the central parts of the structure is 2.3-2.4 $\AA$, in excellent agreement with the separation observed between the gallium surface metallic bi-layers of $2.357 \AA$ [15].

Further exploring this structural motif, the difference between the lowest energy PT-derived $\mathrm{Ga}_{33}^{+}$structure and the $\mathrm{GM} \mathrm{Ga}_{34}^{+}$is the single adatom protruding from the surface of one of the 33-atom planes. This adatom may have a destabilizing effect on the surface structure of the 33atom cluster. This structural destabilization, coupled with the three lowenergy isomers with similar cohesive energy, could contribute to the low, broad melting transition and lower $T_{m}(60 \mathrm{~K}$, by experimental measurement) for the 34-atom cation compared to the 33-atom cation. This argument is also supported by the results and discussion of the extensive GM study reporting this lowest energy $\mathrm{Ga}_{34}^{+}$structure [46].

Given that the PT simulation did not explore the lowest energy structure for the 34-atom cluster, it is difficult to make comparisons to other simulated cluster sizes. Although the lowest energy structure at zerotemperature is not always the most stable structure at finite temperature, the fact that PT does not identify this GM structure raises the issue of convergence, as discussed in more detail in the following section. 


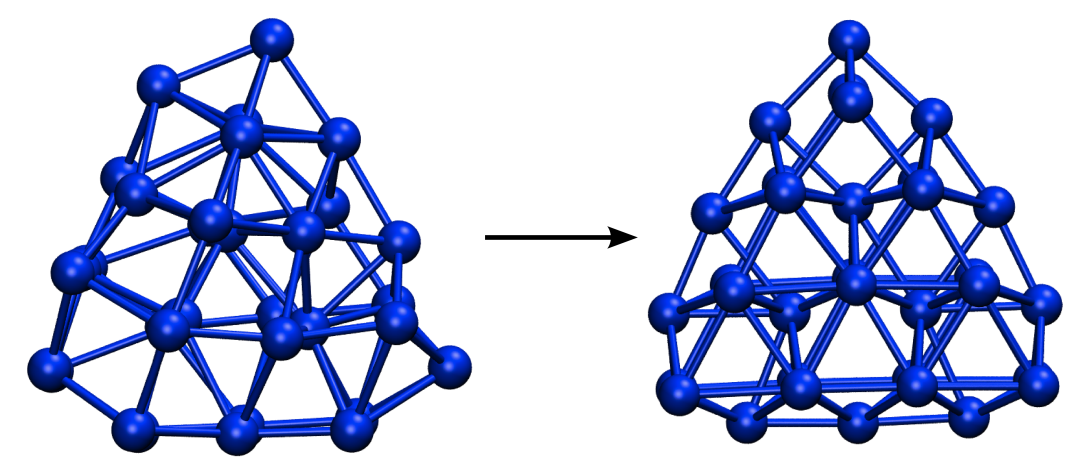

(a) $\mathrm{Ga}_{33}^{+}$

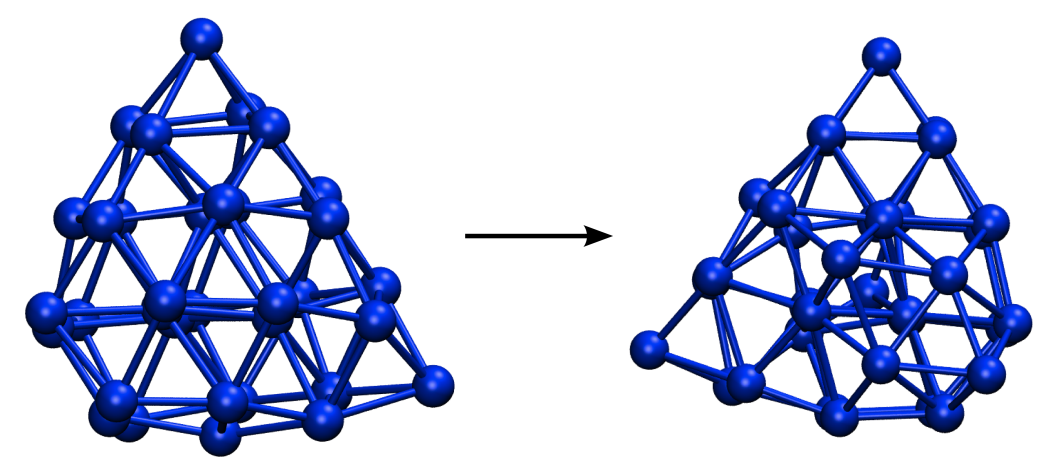

(b) $\mathrm{Ga}_{34}^{+}$

Figure 7.8. PT-derived lowest energy structures (right of the arrow) for the 33- and 34-atom cations, each showing a notable structural change from its initial geometry (left of the arrow). Atomic coordinates are given in Tabs. B.35 and B.36. 


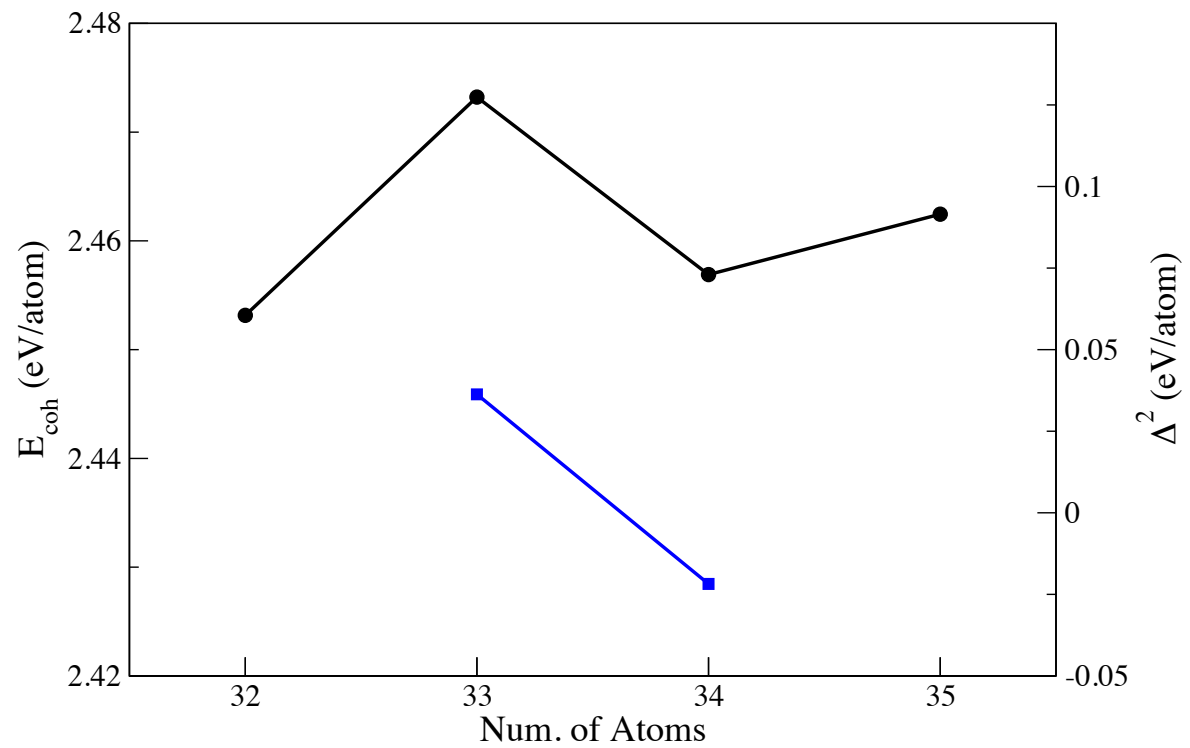

Figure 7.9. Cohesive energies (black) and second order differences (blue) for the lowest energy PT-derived structures.
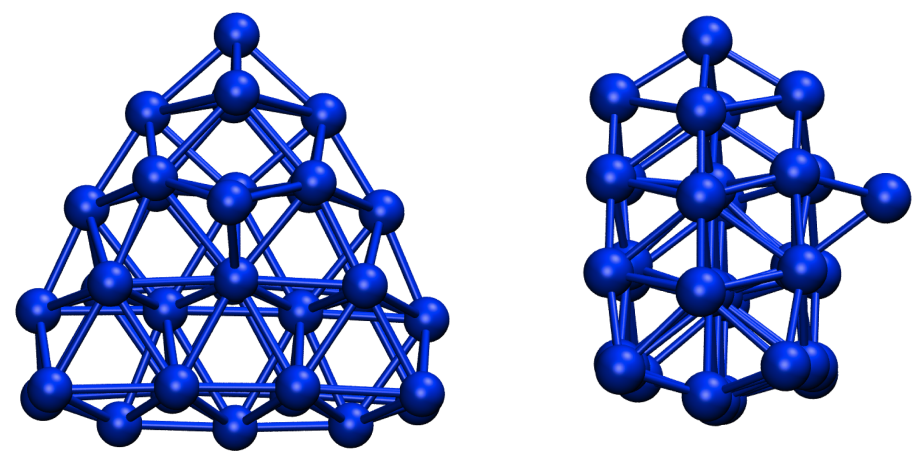

Figure 7.10. The GM structure for $\mathrm{Ga}_{34}^{+}$, obtained through an extensive GM search [46]. Atomic coordinates are given in Tab. B.37. 


\begin{tabular}{cccccc} 
Cluster & $\mathbf{T}_{\mathbf{m}}$ & $\mathbf{E}_{\mathbf{m}}$ & $\Delta \mathbf{T}_{\mathbf{m}}$ & $\Delta \mathbf{E}_{\mathbf{m}}$ & Sim. Time \\
\hline $\mathrm{Ga}_{32}^{+}$ & 510 & -2.29 & -0.096 & $-2.12 \mathrm{e}-6$ & 206 \\
$\mathrm{Ga}_{33}^{+}$ & 630 & -2.29 & -0.014 & $2.05 \mathrm{e}-6$ & 187 \\
$\mathrm{Ga}_{34}^{+}$ & 356 & -2.36 & -0.069 & 0.0 & $397^{*}$ \\
$\mathrm{Ga}_{35}^{+}$ & 448 & -2.34 & 0.280 & $5.82 \mathrm{e}-6$ & $62^{*}$ \\
\hline
\end{tabular}

* qualitatively not converged.

Table 7.2. Summary of the key thermodynamic quantities and convergence measures. The units are $\mathrm{K}$ for temperature, $\mathrm{eV} /$ atom for energy, and ps for simulation time. $\Delta \mathbf{T}_{\mathbf{m}}$ is the change per $\mathrm{ps}$ in the temperature of the canonical specific heat curve peak, given in $\mathrm{K} / \mathrm{ps}$, and $\Delta \mathrm{E}_{\mathrm{m}}$ is the microcanonical analogue in $\mathrm{eV} /$ atom/ps. The total simulation time excludes the initial 10 ps canonical equilibration. While the 34- and 35-atom clusters meet the quantitative convergence criteria, they fail to meet the qualitative criteria.

\subsection{Convergence}

The convergence criteria for each of the large cationic simulations are the same as for previous sizes, outlined in greater detail in Sec. 5.4. For each cluster, the temperature and energy of the maximum specific heat value, relative convergence and total simulation time are given in Tab. 7.2. Each of the simulations is very well-converged with respect to the quantitative criteria, which requires a change of less than $10 \mathrm{~K}$ and $0.01 \mathrm{eV} /$ atom in the melting temperature and energy, respectively, over 10 ps. However, both the $\mathrm{Ga}_{34}^{+}$and $\mathrm{Ga}_{35}^{+}$simulations demonstrate notable variation in the shape and height of the specific heat peaks over a 10 ps interval. For this reason, they fail to meet the qualitative criteria requiring very little change in the overall nature of the specific heat curves over the convergence window.

With only one-third of the simulation time of the next shortest simu- 
lation, it is expected that the $\mathrm{Ga}_{35}^{+}$simulation would converge with additional simulation time. However, it is interesting to note that the nonconverged 34-atom simulation ran for nearly double the time of any other simulation. This likely relates to the GM structure differing from both the initial and PT-derived structures. In order to further explore the contributions to structural stability relating to both convergence and the interesting thermodynamic behavior observed for these larger cations, an electronic structure analysis is completed for each of the large clusters.

\subsection{Electronic structure}

For each of the large cationic clusters, Bader, electron localization function (ELF) and spherical harmonic projected electronic density of states (SH-PDOS) analyses were completed for the lowest-energy PT-derived structures. Overall, electronic structure analysis revealed identical trends to those found in the 20-atom clusters. As illustrated in Fig. 7.11, the large cationic clusters exhibit charge polarization with negatively charged central atoms surrounded by neutral or positively charged protrudingsurface atoms. Although the plots are not included, an analysis of the mean square displacement for each cluster size revealed that these negative central atoms were also significantly less mobile at low energies, identical to the results for the 20-atom cluster analysis.

Fig. 7.12 illustrates the results of the ELF analysis for each of the large cationic clusters. This calculation revealed surface-dominated electron localization, with low electron localization in the centers of the clusters. There is no evidence of covalent bonding for any of the large cations, as was previously observed for the 20-atom and microclusters.

The electronic shell structure, as evidenced by the ELF analysis, allows for the jellium-like treatment of electronic structure in a spherical harmonic analysis of the delocalized electronic shells. SH-PDOS for each of the PT-derived structures is illustrated in Fig. 7.13. This analysis shows 
a clear progression in the angular momentum character of the electronic shell structure at low energies. At the HOMO-LUMO gap, however, none of the clusters in this size range exhibit a clear angular momentum character. All clusters are metallic, with a low DOS at the Fermi level. As Bader, ELF and SH-PDOS analysis reveal similar trends for all large cationic clusters, no further insights can be provided as to the odd-even cohesive energy variations or one-atom thermodynamic differences noted across the size range. 


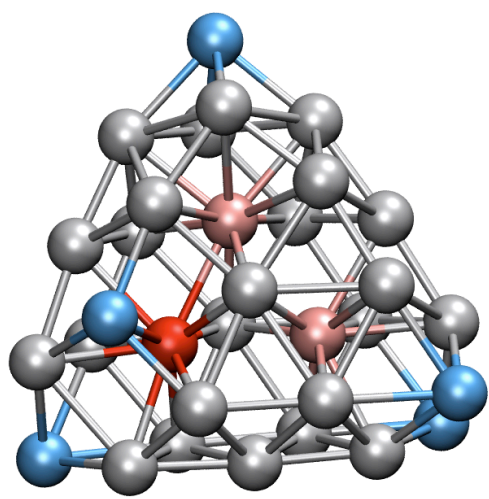

(a) $\mathrm{Ga}_{32}^{+}$

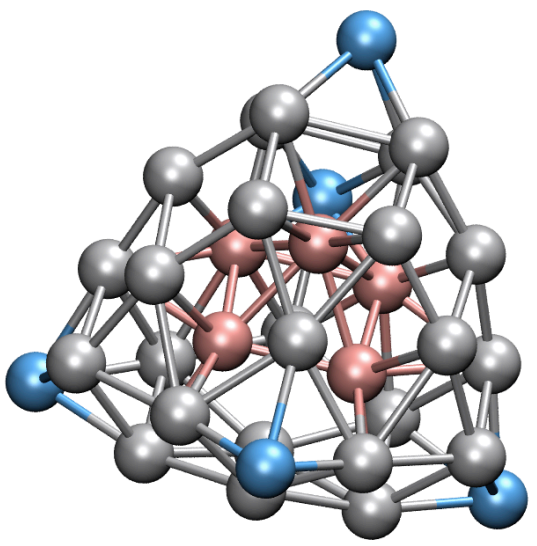

(c) $\mathrm{Ga}_{34}^{+}$

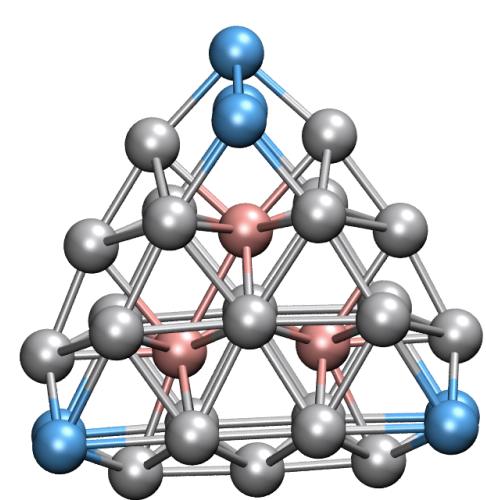

(b) $\mathrm{Ga}_{33}^{+}$

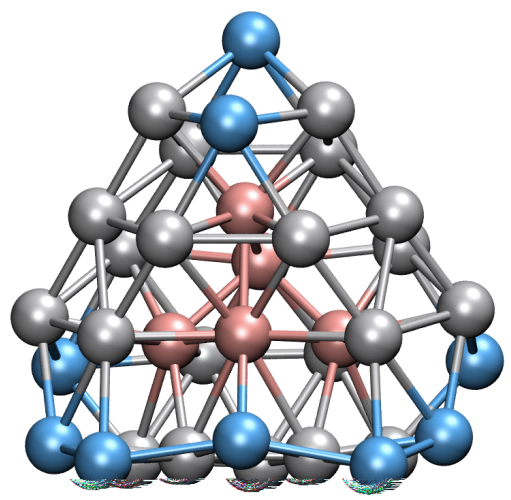

(d) $\mathrm{Ga}_{35}^{+}$

Figure 7.11. Bader analysis results for each of the large clusters: red $=q<$ $-0.2 e^{-} ;$pink $=-0.2 e^{-}<q<-0.1 e^{-}$; silver $=-0.1 e^{-}<q<0.1 e^{-} ;$light blue $=$ $0.1 e^{-}<q<0.2 e^{-}$. 


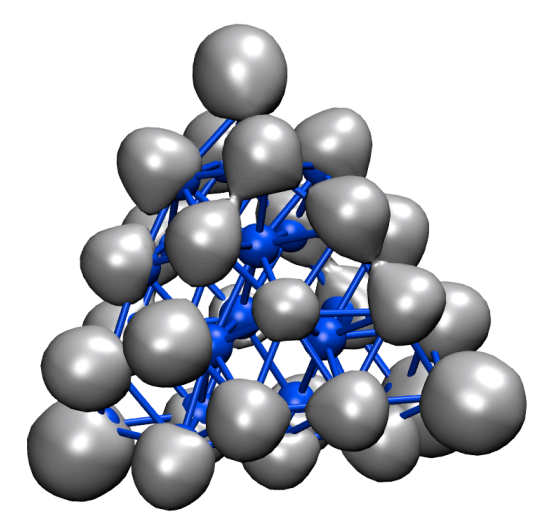

(a) $\mathrm{Ga}_{32}^{+}$

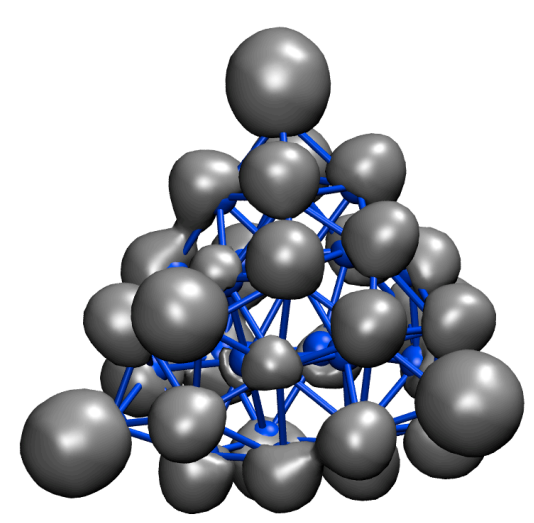

(c) $\mathrm{Ga}_{34}^{+}$

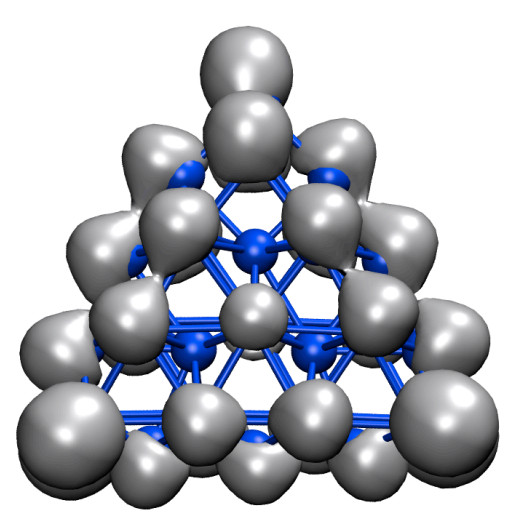

(b) $\mathrm{Ga}_{33}^{+}$

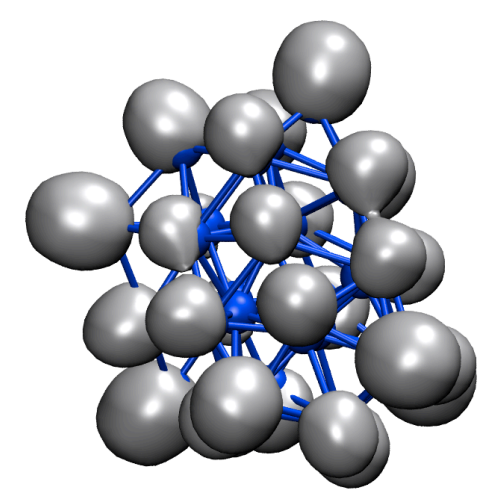

(d) $\mathrm{Ga}_{35}^{+}$

Figure 7.12. ELF analysis for each of the PT-derived lowest energy structures at an isovalue of 0.6. 


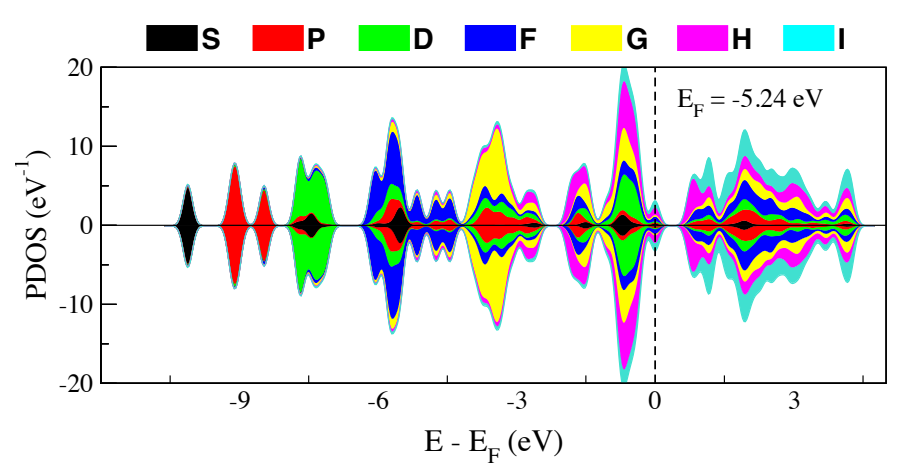

(a)

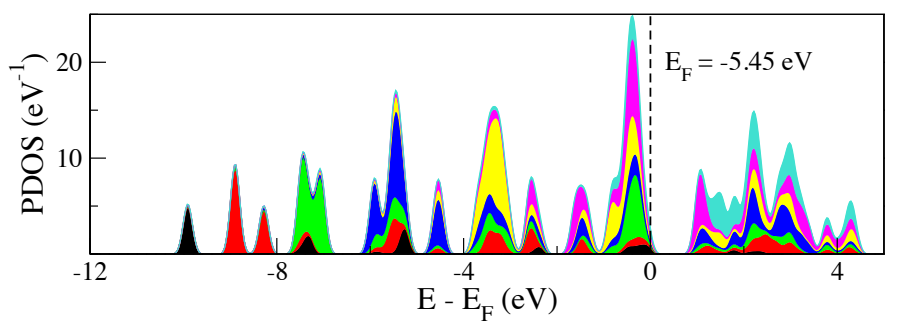

(b)

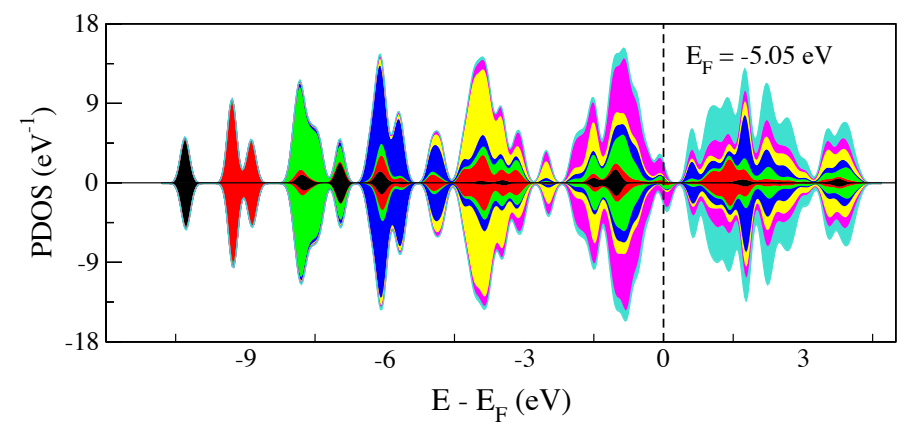

(c)

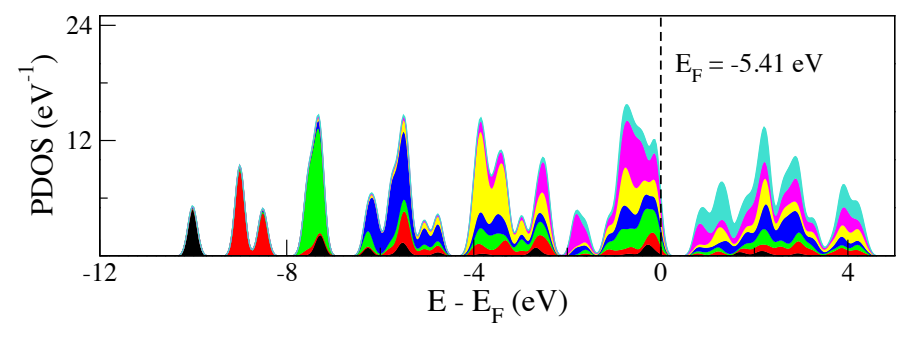

(d)

Figure 7.13. The SH-PDOS for the PT-derived lowest energy structures for (a) $\mathrm{Ga}_{32}^{+}$, (b) $\mathrm{Ga}_{33}^{+}$, (c) $\mathrm{Ga}_{34}^{+}$and (d) $\mathrm{Ga}_{35}^{+}$, at a radius $=5.0 \AA$ from the center of mass. 


\subsection{Summary}

Microcanonical FPMD parallel tempering simulations were completed for the $\mathrm{Ga}_{32}^{+}, \mathrm{Ga}_{33}^{+}, \mathrm{Ga}_{34}^{+}$and $\mathrm{Ga}_{35}^{+}$clusters using the LC pseudopotential model. Overall, the canonical specific heat curves for the 32- and 33-atom clusters best match the experimental results. There are $\sim 75 \mathrm{~K}$ shifts in the simulated melting temperatures as compared to experimental values, although the shifts go in opposite directions for the two cluster sizes. The 32-atom melting temperature is higher than that measured experimentally while the $T_{m}$ for the 33-atom cluster is lower. The origin of these differences has not yet been elucidated.

The microcanonical specific heats match the overall nature of the canonical specific heats for the 32-, 33- and 35-atom simulations. The differences observed for $\mathrm{Ga}_{34}^{+}$likely arise from the lack of convergence for this simulation. Non-convergence could also account for the higher energy peaks observed in both the 34- and 35-atom microcanonical specific heats.

PT identifies lower energy structures for the 33- and 34-atom simulations than their initial configurations. The putative GM structure for $\mathrm{Ga}_{34}^{+}$, however, is not explored during the course of the simulation. This likely accounts for the nearly doubled simulation time without qualitative convergence.

Cohesive energy analysis revealed the same odd-even trend for this size range as observed for the microclusters, with the higher $E_{c o h}$ corresponding to clusters with even numbers of electrons (odd-sized cations). This odd-even trend is not well-correlated with higher melting temperatures or increased structural stabilities, as determined by measures of $\delta_{r m s}$.

Bader, ELF and SH-PDOS analyses reveal nearly identical trends to those noted for the 20-atom and microcluster analyses. Bader analysis revealed a strong charge polarity between the central and outer atoms. ELF analysis demonstrates ion-centered electron localization with no evidence of covalent bonding. SH-PDOS analysis illustrated a clear progression to 
the electronic shell structure at low energies, but no clear nature at the HOMO-LUMO gap. All clusters are metallic, with a low density of states at the Fermi level.

A single atom structural difference between the 33- and 34-atom lowest energy structures may contribute to both a lower melting temperature and change in the melting transition. While these structures are far from bulklike sizes, the triple-plane structural motif is quite reminiscent of metallic surfaces of bulk gallium [15]. As scanning tunneling microscopy measurements of these surfaces have suggested that these metallic surfaces remain solid even after bulk atoms have become molten [24, 23], the bulk-like metallic surface motif may go some way towards explaining the greaterthan-bulk melting in these small gallium clusters.

As evidenced by a single atom vastly altering thermodynamic behavior in these small clusters, the nature of bonding and its effect on the properties of a system is a complex problem. In order to further explore the nature of bonding in molecular solids, we turn now to a method of increments investigation of the solid halogens. The details of these calculations and a summary of the results are provided in the following chapter. 


\section{Chapter 8}

\section{Solid Halogens}

In addressing the exchange and correlation energy of the solid halogens $\mathrm{Cl}_{2}, \mathrm{Br}_{2}$ and $\mathrm{I}_{2}$, only two methods are computationally feasible for systems of this size: DFT and the method of increments (MI). Calculations were completed for both methods using a combination of the quantum chemical codes MOLPRO [178], CRYSTAL09 [179, 180] and CRYSCOR [181, 182] in order to determine the n-body correlation contributions to the bulk cohesive energy of the solid halogens. The following sections describe the components to each method as well as the results.

\subsection{DFT for Solid Halogens}

In order to verify whether the comparably simple DFT method would sufficiently model the cohesive energies of the solid halogens, a set of DFT calculations were completed for solid $\mathrm{Cl}_{2}$ using a variety of functionals. The set of functionals included were the GGA's PW91 [127] and PBE [105], and the hybrid functionals B3LYP [108] and PBE0 [183]. Additionally, dispersion corrections [184] were added for PBE (PBE+d), as well as B3LYP with two different scaling factors: the traditional $s_{6}=1.05(\mathrm{~B} 3 \mathrm{LYP}+\mathrm{d})$, and $s_{6}=1.0\left(\mathrm{~B} 3 \mathrm{LYP}+\mathrm{d}^{*}\right)$. DFT calculations employed CRYSTAL09, using Stuttgart's energy-consistent, multi-electron fit, quasi-relativistic PP [185] 
with a $[\mathrm{Ne}]$ core, while the chemically active, valence electrons were represented by valence triple- $\zeta$ contracted Gaussian type orbital (CGTO) basis sets.

As the Stuttgart library does not have a standard triple $-\zeta$ basis set for chlorine, the triple- $\zeta$ basis utilized for all chlorine calculations was derived from a quadruple- $\zeta$ basis set optimized for the Stuttgart PP. For the $s$ - and $p$-functions, the quadruple- $\zeta$ basis was reduced to triple- $\zeta$ quality by reoptimizing the coefficients in a multi-configurational self-consistent field (MC-SCF) calculation for a single chlorine atom. The $d$ - and $f$-functions were obtained from Dunning's aug-cc-pVTZ basis [186]; however, it should be noted that for DFT calculations, the last $d$-function was eliminated and the $f$-functions were not used. This basis set used for the $\mathrm{Cl}_{2}$ DFT calculations is given in Tab. C.1.

For each functional, a bulk calculation was completed to yield the total energy per unit cell. In order to correct for the basis set superposition error (BSSE), a counterpoise energy calculation was completed. This was accomplished by replacing each atom with only the PPs and basis sets (ghost atoms) within a spherical radius ranging from 3.259 - $10 \AA$, yielding a BSSE correction to the total cohesive energy as a function of radius. For non-dispersion corrected functionals, the total cohesive energy was determined as

$$
E_{c o h}\left(r_{\mathrm{BSSE}}\right)=E_{\text {bulk }}-E_{\mathrm{CP}}\left(r_{\mathrm{BSSE}}\right),
$$

where $E_{\mathrm{CP}}$ represents the counterpoise calculation. For each functional including dispersion corrections, CRYSTAL09 automatically accounts for the dispersion energy within the bulk calculation. The counterpoise calculation for the BSSE corrections remained unchanged; however, an additional calculation for the dispersion energy of only the two molecules within the unit cell was also necessary, yielding $E_{\text {disp }}^{\mathrm{UC}}$. For the functionals including dispersion corrections, the cohesive energy was calculated as 


$$
E_{c o h}\left(r_{\mathrm{BSSE}}\right)=E_{\mathrm{bulk}}-E_{\mathrm{CP}}\left(r_{\mathrm{BSSE}}\right)-E_{\mathrm{disp}}^{\mathrm{UC}}
$$

A summary of the results is given in Fig. 8.1, with the table to the right listing the converged values for each functional. The cohesive energies converge with BSSE corrections at a radius of $\sim 5.5 \AA$. Not only do the cohesive energies vary greatly depending on the functional, none come close to the literature value. This variety of cohesive energies is similar the results of DFT calculations for solid mercury [187, 111, 112], further verifying that DFT calculations would be insufficient to model the exchangecorrelation interactions within the vdW-bound halogen crystals. In order to more accurately calculate the cohesive energy for the solid halogens in a systematically improvable way, the method of increments is employed. This method is outlined in greater detail in the following sections.

\subsection{Incremental Calculations}

The additive correlation energy for solids can be calculated according to highly accurate, post-HF methods by using the method of increments (MI). As described in Sec. 2.5, this is accomplished by assuming the effects of electron correlation are localized to a small region of the crystal surrounding each correlated subunit and calculating the correlation energies only for these smaller regions. Initial testing revealed that the use of linear scaling approximations (local and density-fitting) changed the correlation energies by $\sim 1 \mu \mathrm{H}$ compared to $\operatorname{CCSD}(\mathrm{T})$ and MP2. As the linearscaled methods are considerably more efficient, all incremental calculations were completed using MOLPRO's implementation of density-fitting, local CCSD(T) (DF-LCCSD(T)) and density-fitting, local MP2 (DF-LMP2). The following sections outline MI calculations for the solid halogens, to include a description of basis sets and pseudopotentials, embedding schemes, orbital domains and local coupled-cluster excitation approximations. 


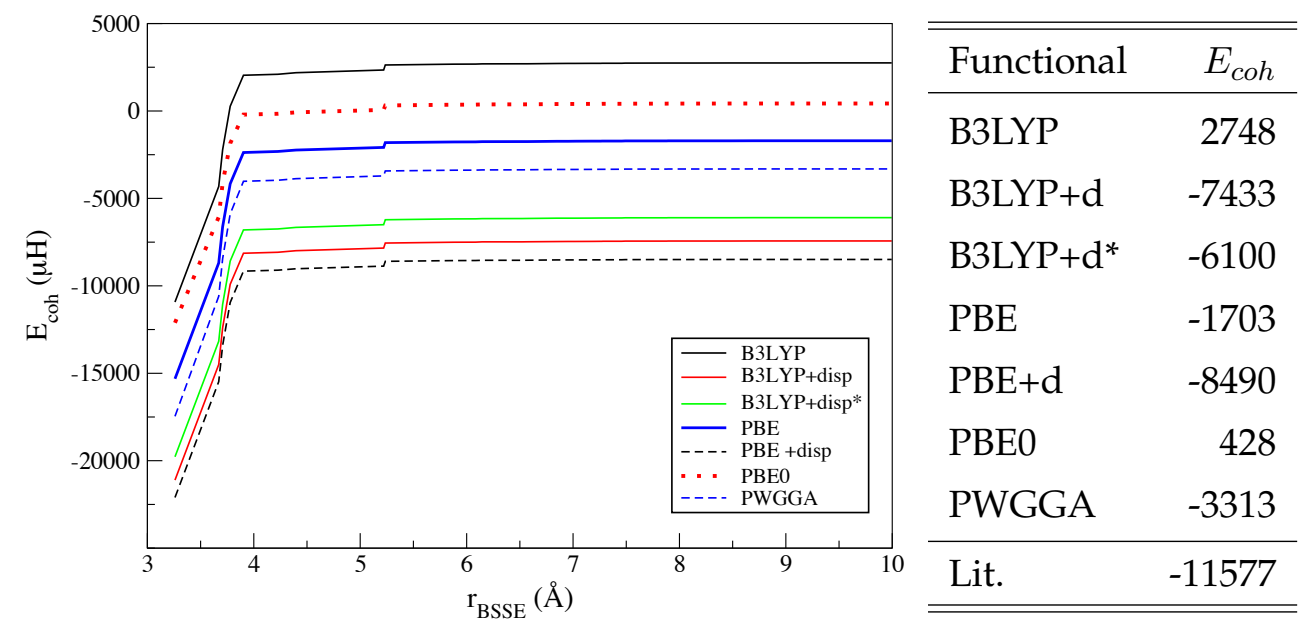

Figure 8.1. Left: Plot of the cohesive energy of solid chlorine, calculated correcting for BSSE within a range of radii, $r_{\mathrm{BSSE}}$, surrounding the unit cell. Right: Table showing the variety of converged cohesive energies (in $\mu \mathrm{H})$ for each functional and functional+dispersion, as compared to the literature value [76]. Although this value is zero point corrected in the literature, that correction has been removed so as to be directly comparable to the calculated, non-zero point corrected DFT cohesive energies.

\subsubsection{Crystal structure}

The crystallographic unit cell parameters for $\mathrm{Cl}_{2}, \mathrm{Br}_{2}$ and $\mathrm{I}_{2}$ were obtained from Refs. [188, 188, 189], respectively, and are summarized in Tab. 8.1. This table also gives the intra-molecular (dimer) separation $\left(\mathbf{r}_{12}\right)$, the shortest inter-molecular distance (as measured between centers of dimers, $\mathbf{R}_{\mathbf{1 2}}$ ) and the atomic second nearest neighbor $(\mathrm{NN})$ distance $\left(\mathrm{r}_{2 \mathrm{NN}}\right)$. In order to better illustrate trends, the dimer-to-intermolecular ratio $\left(\mathbf{r}_{12} / \mathbf{R}_{12}\right)$ and second NN-to-intermolecular ratio $\left(\mathbf{r}_{2 \mathrm{NN}} / \mathbf{R}_{12}\right)$ are also calculated. From low to high atomic number, the first ratio shows an increasing intramolecular separation relative to the intermolecular separation, possibly indicating a decreasing degree of covalency. The second ratio illustrates the decreasing second $\mathrm{NN}$ distance relative to the intermolecular separation. 
Taken together, both ratios indicate an increasingly compact structure relative to the intramolecular bond distance.

The crystal structure of gallium is also included for comparison. The dimeric separation is slightly larger than that of bromine, while it has the smallest intermolecular separation. This results in an increased dimer ratio relative to the intermolecular separation, along with a larger relative second NN separation. As the number of nearest neighbors would scale with this ratio, a correlation between this ratio and metallicity, which increases for the solid halogens with increasing atomic number [75], would be logical.

As previously mentioned in Section 2.5, each of the centers used in MI can correspond to an atom, bond or molecule, where the selection is typically based on the structure of the solid. The crystal structure of the solid halogens is comprised of sets of dimers, and could almost be described as a molecular face-centered cubic structure where each of the vertices consists of a tilted $X_{2}$ molecule where $X=\{\mathrm{Cl}, \mathrm{Br}, \mathrm{I}\}$. Each row of the crystal has oppositely tilted dimers, as illustrated in Fig. 8.2. This dimeric crystal structure leads to the obvious choice of molecular centers consisting of an $\mathrm{X}_{2}$ dimer.

MI yields the total correlation energy contribution per unit cell. Belonging to space group 64, the symmetric crystal structure of the solid halogens greatly simplifies the calculations. As demonstrated in Fig. 8.3 (a), the primitive unit cell for the crystalline halogens consists of a single molecule with two additional atoms just below and above the center of the dimer bond. Symmetry allows this unit cell to be shifted so that it will contain two full molecules, as shown in Fig. 8.3 (b). As the first index, $i$, shown in Eq. (2.61) has to be calculated for every center in the unit cell, calculations over $i$ for the solid halogens need only include one center, with the other molecule in the unit cell included by symmetry. 


\begin{tabular}{lcccccc}
\hline \hline Halogen & Unit Cell & $\mathbf{r}_{\mathbf{1 2}}$ & $\mathbf{R}_{\mathbf{1 2}}$ & $\mathbf{r}_{\mathbf{1 2}} / \mathbf{R}_{\mathbf{1 2}} \%$ & $\mathbf{r}_{\mathbf{2 N N}}$ & $\mathbf{r}_{\mathbf{2 N N}} / \mathbf{R}_{\mathbf{1 2}} \%$ \\
\hline \multirow{2}{*}{$\mathrm{Cl}_{2}$} & 6.1453 & & & & & \\
& 4.3954 & 1.994 & 3.778 & $53 \%$ & 3.258 & $86 \%$ \\
& 8.1537 & & & & & \\
$\mathrm{Br}_{2}$ & 6.5672 & & & & & \\
& 4.4678 & 2.301 & 3.971 & $58 \%$ & 3.286 & $83 \%$ \\
& 8.6938 & & & & & \\
$\mathrm{I}_{2}$ & 7.255 & & & & & \\
& 4.795 & 2.703 & 4.348 & $62 \%$ & 3.538 & $81 \%$ \\
\hline & 9.780 & & & & & \\
$\mathrm{Ga}$ & 4.523 & & & & & \\
& 4.524 & 2.484 & 3.199 & $77 \%$ & 2.691 & \\
\hline \hline
\end{tabular}

Table 8.1. The crystallographic structure, with unit cell parameters for solid chlorine [188], bromine [188] and iodine [189]. The dimer separation $\mathrm{r}_{12}$, shortest intermolecular separation $\mathrm{R}_{12}$ (measured from the centers of two dimers) and atomic 2nd NN distance are given for each halogen, including the ratio of the dimer-to-intermolecular ratio and 2 nd NN-tointermolecular ratio. For comparison, each of these parameters is included for gallium $[190,5]$. 


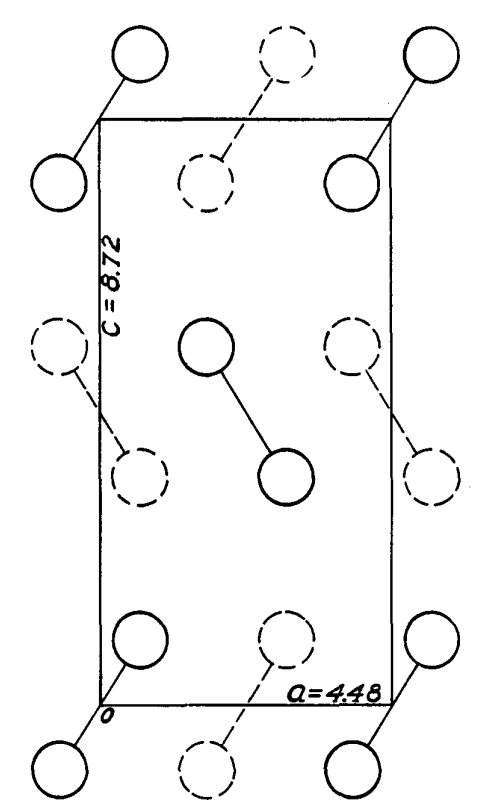

Figure 8.2. The FCC-like molecular structure of solid bromine, reproduced from Ref. [67]. The solid structure of chlorine, iodine and gallium are identical, but with different lattice parameters $a, b$ and $c$.

\subsubsection{Basis sets and pseudopotentials}

For later ease of representation, the basis sets and pseudopotentials (PP) utilized in the method of increments calculations will be introduced here. All PP's and basis sets were obtained from the Stuttgart-Cologne group and differed according to the halogen. The incremental calculations for each solid halogen utilized a large-core (LC) model, treating all $d$-electrons as part of the chemically inactive core, with only the 7 outermost $s^{2} p^{5}$ electrons as valence. For $\mathrm{Br}_{2}$, a second small-core (SC) PP model was also employed to test the effect of the $d$-electrons, treating the $3 s^{2} 4 d^{10} 3 p^{6} 4 s^{2} 4 p^{5}$ electrons as valence.

Each of the LC models utilized an energy-consistent, multi-electron fit, quasi-relativistic PP [185], with a chemically inactive [Ne] core for chlo- 


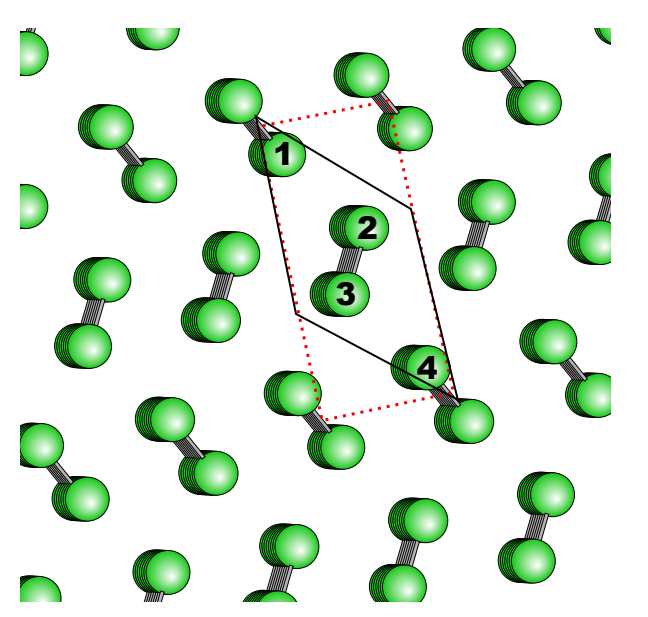

(a)

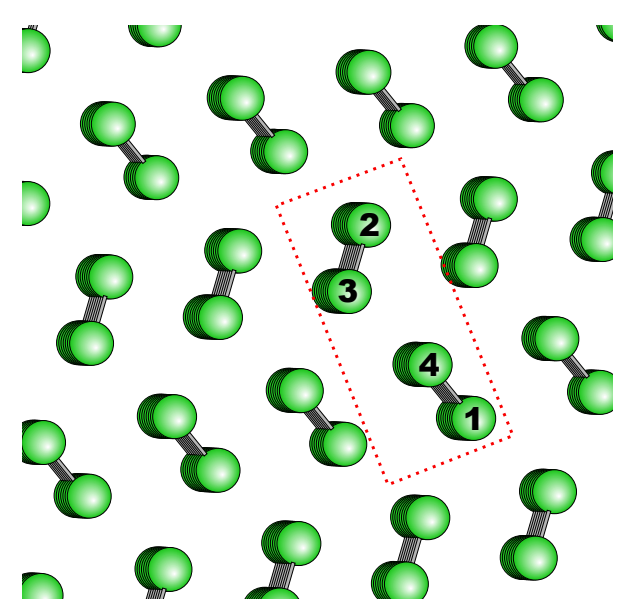

(b)

Figure 8.3. Illustration of the crystal structure of the solid halogens with unit cell atoms numbered 1-4, demonstrating: (a) an approximation of the primitive unit cell (black solid) and the unit cell comparable to Fig. 8.2 (red dotted); (b) the shifted unit cell that includes two full molecules.

rine, $[\mathrm{Ar}] 3 d^{10}$ core for bromine and $[\mathrm{Kr}] 4 d^{10}$ core for iodine. The valence electrons not included as core were represented by contracted Gaussian type orbital (CGTO) sets. For chlorine, these sets were derived as outlined in Sec. 8.1; however, for MI calculations, all $d$ - and $f$-functions were utilized. For bromine and iodine, these sets were obtained from the Dunning's augmented valence triple- $\zeta$ basis sets, optimized for the Stuttgart pseudopotentials [185]. Testing for $\mathrm{Cl}_{2}$ confirmed that the same basis set type at the double- $\zeta$ level was insufficient to capture the requisite level of accuracy. The basis sets used for the incremental calculations of solid $\mathrm{Cl}_{2}$, $\mathrm{LC} \mathrm{Br}_{2}$ and $\mathrm{I}_{2}$ are given in Tabs. C.3, C.4 and C.5.

For $\mathrm{Br}_{2}$, the small-core (SC) PP model used an energy-consistent, multielectron fit, fully-relativistic PP, taking a core of [Ne] [191]. Augmented, triple- $\zeta$ correlation-consistent basis sets accounting for molecular core-valence interactions (aug-cc-pwCVTZ) were used to describe the non-core elec- 
trons [192]. This basis set is summarized in Tabs. C.6, C.7 and C.8. The SC PP and basis sets were only utilized for the correlated molecules. The LC basis set, as given in Tab. C.4, was used for the uncorrelated molecules.

For both LC and SC models, $d-, f$ - and (for the SC $\mathrm{Br}_{2}$ model) $g$-functions were taken into account for the correlated centers. However, testing for $\mathrm{Cl}_{2}$ confirmed that they contributed insignificantly to the correlation energy when utilized for the frozen-HF clusters and were, thus, excluded from the embedding basis sets. The details of the embedding scheme are outlined in the following section.

\subsubsection{Embedding}

MI assumes that the electron-electron correlation effects are localized, such that only a local environment surrounding each center needs to be considered for the correlation calculations. However, determining the extent of that local environment required additional testing. For the solid halogens, a cluster of atoms that included sets of nearest neighbors (NN) was selected as the embedding environment.

A number of tests were completed in order to determine the number of NN required in order to most accurately capture the electron correlation energy while still keeping the computational cost to a minimum. These included calculations for the first through sixth $\mathrm{NN}$ of the one-center increment and the first through fourth $\mathrm{NN}$ of the two-center increment. The two-center used for testing had both centers contained within the unit cell, as this increment likely represents the largest correlation contribution to the cohesive energy. These tests were performed only for $\mathrm{Cl}_{2}$, as it was the computationally simplest system and taken to be representative of the other halogens. The calculations were completed using MOLPRO, with basis set for chlorine as outlined in the previous section.

Tables 8.2 and 8.3 give an overview of the numbers of NN and corresponding incremental energies. Due to the poor convergence of the one- 
center increments with increasing numbers of NN, it was determined that all molecules up to and including the sixth NN would be necessary in order to adequately capture the correlation contribution for the one-centers. Although it appears that inclusion of the third NN yields sufficient convergence for the two-centers, a geometric consideration led to a different conclusion. This argument stemmed from a visual inspection of the chlorine crystal structure, observing the geometric progression of nearest neighbor additions surrounding a center molecule as represented in Fig. 8.4. A shell-closing was observed after the fourth set of NN were added, which led to the decision that all molecules up to and including the fourth NN would be used for the two-center calculations.

\begin{tabular}{cccc}
\hline$\epsilon_{1}(\mu H)$ & Num. of Mol. & DF-LCCSD(T) & DF-LMP2 \\
\hline \hline NN1 & 5 & 941 & 916 \\
NN2 & 7 & 1103 & 1077 \\
NN3 & 11 & 2556 & 2245 \\
NN4 & 15 & 2802 & 2482 \\
NN5 & 17 & 2800 & 2479 \\
NN6 & 25 & 2797 & 2473 \\
\hline
\end{tabular}

Table 8.2. Summary of one-center incremental correlation energies for different sets of $\mathrm{NN}$. The ' $\mathrm{NN} x$ ' designation gives the sets of $\mathrm{NN}$ up to and including the $x$ th set; i.e., NN6 is the set of NN up to and including the 6 th NN. The number of molecules listed in the second column is the total number, to include the one correlated center molecule. 


\begin{tabular}{rccc}
\hline$\epsilon_{12}(\mu H)$ & Num. of Mol & DF-LCCSD(T) & DF-LMP2 \\
\hline \hline NN1 & 8 & -3811 & -4427 \\
NN2 & 10 & -3810 & -4425 \\
NN3 & 18 & -3740 & -4357 \\
NN4 & 22 & -3735 & -4352 \\
\hline
\end{tabular}

Table 8.3. Summary of two-center incremental correlation energies for different sets of $\mathrm{NN}$. The ' $\mathrm{NN} x$ ' designation is generally the same as for the one-centers, although it now represents the number of NN for each of the two individual centers. The number of molecules listed in the second column is the total number, accounting for only once for molecules falling into both sets of NN and including the two correlated center molecules. 


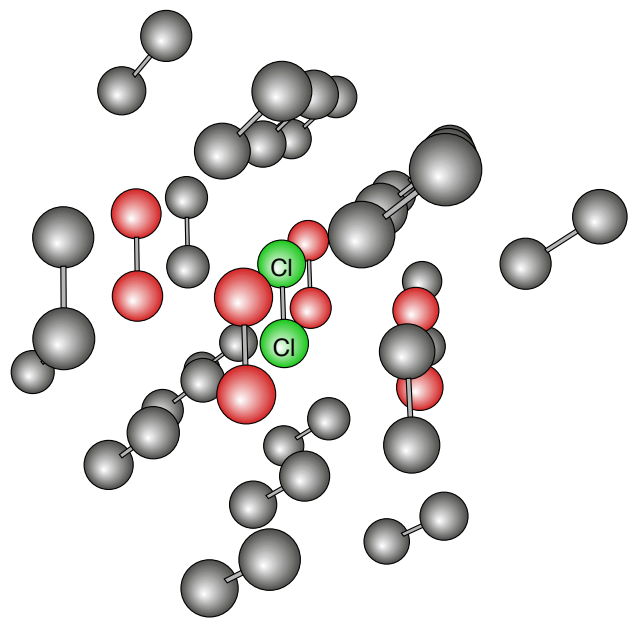

(a)

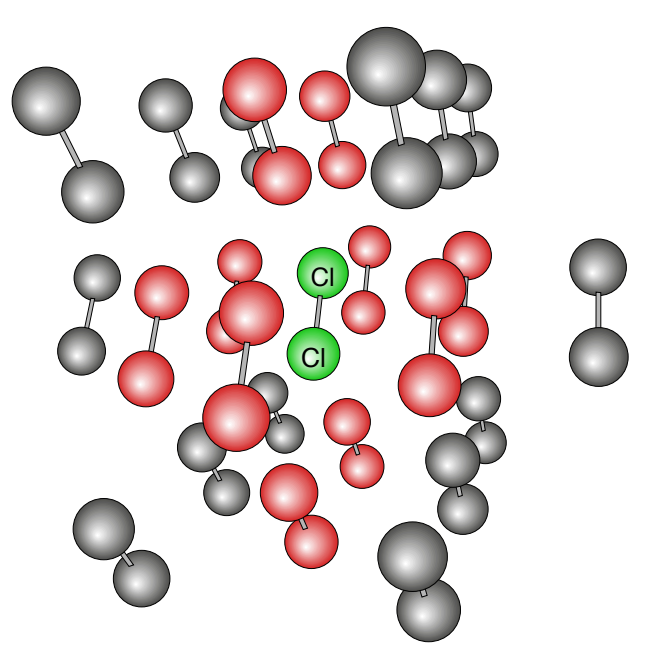

(c)

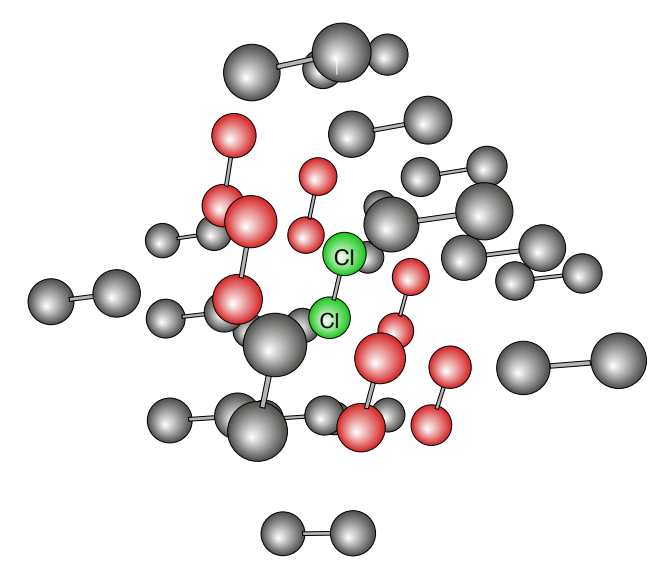

(b)

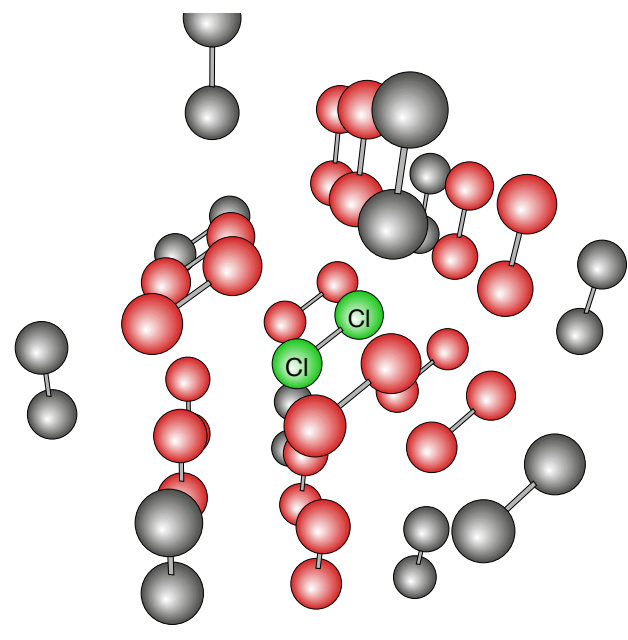

(d)

Figure 8.4. The crystal structure of chlorine illustrating the sets of NN for a central molecule (in green). The atoms in red represent the set of (a) first NN, (b) second NN, (c) third NN and (d) fourth NN, illustrating the geometric shell closing for this final set. For each picture, the atoms in grey represent molecules up to and including the set of sixth nearest neighbors not included in the designated set. 


\subsubsection{Orbital domains}

Two methods were employed for selecting the sets of atoms contributing to orbital domains. Originally, a Boughton-Pulay (BP) thresholding scheme $[193,194]$ was utilized. In theory, this method automatically selects each atom such that the orbital domain consisting of all basis functions for that particular atom spans the corresponding localized orbital at a specified accuracy (defined by the threshold value). This is the recommended and default setting within MOLPRO, and was tested for the $\mathrm{Cl}_{2}$ system with settings of 0.99 and 0.998 , each of which exceed the recommended level of accuracy at 0.985 for the augmented triple- $\zeta$ basis set.

Although the thresholding BP method is recommended by MOLPRO, it was found to be insufficient for determining the appropriate orbital domains for the solid halogens. The issue was discovered during one-center $\mathrm{NN}$ testing when energy inconsistencies were noted in the energy progression for increasing $\mathrm{NN}$ for both the $\mathrm{Cl}_{2}$ and $\mathrm{LC} \mathrm{Br}_{2}$ calculations. Upon further investigation, the source of the inconsistencies were traced to orbital domains that both differed between the correlation and counterpoise calculations, and included atoms from the embedding set. Fig. 8.5 (a) and (b) illustrate these issues with orbital domain outputs from an incremental and counterpoise calculation for the same one-center increment.

The problem was solved by reducing the BP threshold to 0.8 and adding an extended domain parameter defining local orbital atom selections by distance. MOLPRO'S parameter REXT designates that any atom found within a defined radius is added to the orbital domain set. Fixing this parameter at $5.0 a_{0}$ allowed the explicit selection of exactly two atoms to each orbital domain, both of which were atoms within the correlated set (as opposed to the embedding set). By adjusting the domain definition to account for only 2 -atoms, the energy inconsistencies were eliminated. Fig. 8.5 (c) and (d) represent the consistent 2-atom domains. 

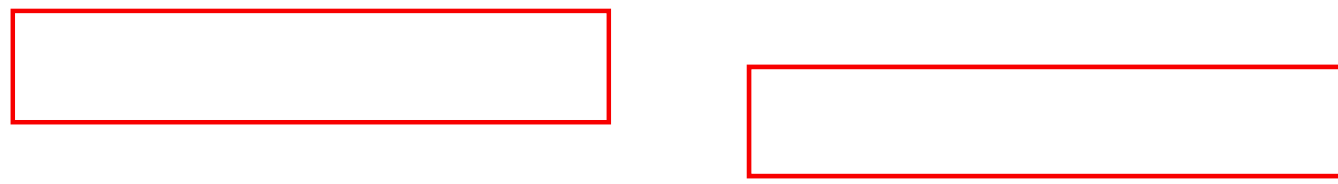

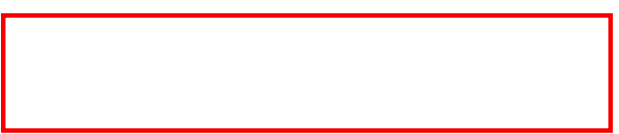

(a) Correlation, BP

Extended orbital domains

$\begin{array}{rrrrr}\text { Orb. } & \text { Atom } & \text { Rmin } & \text { Bonds } & \text { Pairs } \\ 99.1 & 1 \text { CL1 } & 0.00 & 0 & \text { scud } \\ & 2 \text { CL1 } & 3.77 & 1 & \text { scud } \\ 100.1 & 1 \text { CL1 } & 0.00 & 0 & \text { scud } \\ & 2 \text { CL1 } & 3.77 & 1 & \text { scudd } \\ 101.1 & 1 \text { CL1 } & 0.00 & 0 & \text { scudd } \\ & 2 \text { CL1 } & 3.77 & 1 & \text { scudd } \\ 102.1 & 1 \text { CL1 } & 0.00 & 0 & \text { scudd } \\ & 2 \text { CL1 } & 0.00 & 0 & \text { scudd } \\ 103.1 & 1 \text { CL1 } & 3.77 & 1 & \text { scud } \\ & 2 \text { CL1 } & 0.00 & 0 & \text { scud } \\ 104.1 & 1 \text { CL1 } & 3.77 & 1 & \text { scud } \\ & 2 \text { CL1 } & 0.00 & 0 & \text { scud } \\ 105.1 & 1 \text { CL1 } & 3.77 & 1 & \text { scud } \\ & 2 \text { CL1 } & 0.00 & 0 & \text { scud }\end{array}$

(c) Correlation, 2-atom (b) Counterpoise, BP

Extended orbital domains

\begin{tabular}{|c|c|c|c|c|}
\hline Orb. & Atom & Rmin & Bonds & Pairs \\
\hline \multirow[t]{2}{*}{1.1} & 1 CL1 & 3.77 & 1 & scud \\
\hline & 2 CL1 & 0.00 & 0 & scuo \\
\hline \multirow[t]{2}{*}{2.1} & 1 CL1 & 3.77 & 1 & \\
\hline & 2 CL1 & 0.00 & 0 & $\mathrm{scw}$ \\
\hline \multirow[t]{2}{*}{3.1} & 1 CL1 & 3.77 & 1 & 80 \\
\hline & 2 CL1 & 0.00 & 0 & \\
\hline \multirow[t]{2}{*}{4.1} & 1 CL1 & 0.00 & 0 & sch \\
\hline & 2 CL1 & 0.00 & 0 & \\
\hline \multirow[t]{2}{*}{5.1} & 1 CL1 & 0.00 & 0 & $c 010$ \\
\hline & 2 CL1 & 3.77 & 1 & \\
\hline \multirow[t]{2}{*}{6.1} & 1 CL1 & 0.00 & 0 & $\mathrm{sch}$ \\
\hline & 2 CL1 & 3.77 & 1 & \\
\hline \multirow[t]{2}{*}{7.1} & 1 CL1 & 0.00 & 0 & $\mathrm{scl}$ \\
\hline & 2 CL1 & 3.77 & 1 & \\
\hline
\end{tabular}

(d) Counterpoise, 2-atom

Figure 8.5. The selection of atoms attributed to each localized orbital domain for the BP thresholding scheme of the one-center $\mathrm{Cl}_{2}$ (a) correlation and (b) counterpoise calculations. The inconsistencies are outlined as: (red) selected atoms include embedding atoms, with CL1 designating correlated atoms and CL2 as embedding atoms; (blue) orbital domains differ between the correlation and counterpoise calculations. The 2-atom, extended domains illustrated for the (c) correlation and (d) counterpoise calculations demonstrate the domain consistency for this method. 


\subsubsection{Coupled-cluster excitations}

Testing was also completed in order to evaluate the treatment of triple excitations within the coupled-cluster calculations. Comparisons were made between the incremental energies calculated excluding triple excitations (CCSD), using the T0 approximation (CCSD(T0)) and treating the triples explicitly $(\operatorname{CCSD}(\mathrm{T}))$. As illustrated in Tables 8.4 and 8.5, treating only the single and double excitations excludes a substantial portion of the correlation energy for both the one- and two-center increments. Explicit T evaluation contributes significantly to the correlation energy within the onecenter calculations but is negligible for the two-center incremental energies. Explicit T calculations were still completed for all LC, two-center incremental calculations for each of the halogens, as well as the three-center calculations for $\mathrm{Cl}_{2}$, further verifying that the $\mathrm{T} 0$ approximation was sufficient for the two- and three-centers.

\begin{tabular}{ccccc}
\hline$\epsilon_{1}(\mu H)$ & DF-LCCSD & DF-LCCSD(T0) & DF-LCCSD(T) & $\Delta E_{T-T 0}$ \\
\hline \hline NN1 & 782 & 1119 & 941 & -178 \\
NN2 & 915 & 1260 & 1103 & -157 \\
NN3 & 2214 & 2646 & 2556 & -90 \\
NN4 & 2431 & 2848 & 2802 & -46 \\
NN5 & 2429 & 2846 & 2800 & -54 \\
NN6 & 2426 & 2826 & 2797 & -71 \\
\hline
\end{tabular}

Table 8.4. Summary of $\mathrm{Cl}_{2}$ one-center incremental correlation energies comparing various treatment of the triple excitations within local coupled-cluster calculations: DF-LCCSD, single-double excitations only; DF-LCCSD(T0), triples calculated with the T0 approximation; DF$\operatorname{LCCSD}(\mathrm{T})$, triple excitations treated explicitly. The final column gives the energy difference: $\Delta E_{T-T 0}=E_{D F-L C C S D(T)}-E_{D F-L C C S D(T 0)}$. 


\begin{tabular}{ccccc}
\hline$\epsilon_{12}(\mu H)$ & DF-LCCSD & DF-LCCSD(T0) & DF-LCCSD(T) & $\Delta E_{T-T 0}$ \\
\hline \hline NN1 & -3187 & -3788 & -3811 & -23 \\
NN2 & -3186 & -3787 & -3810 & -23 \\
NN3 & -3134 & -3719 & -3740 & -21 \\
NN4 & -3130 & -3713 & -3735 & -22 \\
\hline
\end{tabular}

Table 8.5. Summary of $\mathrm{Cl}_{2}$ two-center incremental correlation energies, comparing various treatment of the triple excitations within local coupled-cluster calculations: DF-LCCSD, single-double excitations only; DF-LCCSD(T0), triples calculated with the T0 approximation; DF$\operatorname{LCCSD}(\mathrm{T})$, triple excitations treated explicitly. The final column gives the energy difference: $\Delta E_{T-T 0}=E_{D F-L C C S D(T)}-E_{D F-L C C S D(T 0)}$.

\subsubsection{Incremental Calculations}

At the conclusion of the testing, incremental calculations were completed for the $\mathrm{Cl}_{2}, \mathrm{LC}$ and $\mathrm{SC} \mathrm{Br}_{2}$, and $\mathrm{I}_{2}$ models according to the following schemes. For the one-center increments, the correlation energy calculation for the central molecule was performed using DF-LMP2 and DF-LCCSD(T). The correlated molecule was embedded in a frozen-HF cluster, consisting of molecules up to and including the 6 th NN.

The two-center incremental calculations were completed using DF-LMP2 coupled with DF-LCCSD(T0) for the $\mathrm{Br}_{2}$ SC model and DF-LCCSD(T) for all LC models. These calculations included all possible geometries with one of the centers contained within the unit cell and the second center located anywhere in the crystal up to a radius of $11.0 \AA, 11.6 \AA$ and 12.7 $\AA$ for $\mathrm{Cl}_{2}, \mathrm{Br}_{2}$ and $\mathrm{I}_{2}$ respectively. These distances were defined so that the numbers and geometries of two-center increments were the same for each halogen. For each center, the set of 4 th $\mathrm{NN}$ were taken to define the embedding cluster, accounting only once for any molecules included in the NN set for both centers. With all possible geometries within that ra- 
dius defined, symmetric geometries were tallied to define a weight. Each unique geometry was calculated only once, with the incremental energy then multiplied by the weight to give its total contribution to the unit cell. For the one geometry with both centers in the unit cell, the correlation energy was included in total. For all geometries with only one center in the unit cell, the correlation contribution was halved. The incremental calculations for each weighted-geometry were then summed to give a total two-center correlation contribution to the cohesive energy.

The three-center incremental calculations were completed in a similar manner to the two-centers, with some distinctions given the new geometric possibilities. The radius cut-off for all $j$ and $k$ centers (as defined in Eq. (2.60)) was the same as for the two centers; however, previous work has shown that the correlation contributions for the three-centers fall off more quickly with increasing center-separation. A requirement was added that specified the average molecular separation of the three molecules be less than $7.0 \AA, 7.5 \AA$ and $7.95 \AA$ for $\mathrm{Cl}_{2}, \mathrm{Br}_{2}$ and $\mathrm{I}_{2}$ respectively. Once again, symmetry was accounted for in weighting each geometry by the number of times it appeared within the subset of the crystal. The correlation contribution was multiplied by $2 / 3$ if two of the three centers were included within the unit cell, and by $1 / 3$ for all remaining geometry combinations. The three-center incremental weighted-energies were summed to give a total three-center increment correlation contribution.

\subsubsection{LC Model Results}

The method of increment results for the $\mathrm{LC} \mathrm{Cl}_{2}, \mathrm{Br}_{2}$ and $\mathrm{I}_{2}$ are listed in Tabs. 8.6, 8.7 and 8.8, respectively. Each of these tables includes the one-, two- and three-center incremental energies for DF-LMP2, DF-LCCSD(T0) and DF-LCCSD(T) (for the one- and two-centers), as well as the sum for each method. Since the full-triples were not evaluated for the three-center calculations, the sum for this column includes the perturbative triples (T0) 
calculation as an approximation. As can be seen from the fourth column, showing the incremental energy change between the T0-approximation and the full-triples approximation, the energy not accounted for in the two-center T0-approximation is minimal compared to the total incremental energy for that increment. It is therefore assumed that the T0 approximation is more than sufficient for the three center calculations.

Additionally listed for each halogen are estimates of the cohesive energies [76]. A comparison of the incremental energies between the three solid halogens shows that they generally increase with increasing atomic number, following the trend of the referenced cohesive energy estimates. It should be noted that although a thorough literature review was completed, no experimental results outlining the cohesive energies for solid chlorine, bromine or iodine could be identified. Ref. [76] lists a private correspondence as the source of the cohesive energies. Additionally, this reference does not actually list the cohesive energies themselves but the zero-point energies, along with the percentage of the cohesive energies that these zero-point energies represent.

From Ref. [77], it could be surmised that Yamasaki and others attempting to theoretically justify the crystal structure of the solid halogens $[70,195]$ make estimates to the cohesive energy based on the heats of sublimation. This method for calculating cohesive energy, however, is solidly refuted in Ref. [196]. Therefore, the values listed in each table as the reference (literature) energies should not be treated as fact.

\subsubsection{SC Model Results}

In order to gauge the correlation contributions from the $d$-electrons, SC calculations were completed for $\mathrm{Br}_{2}$ one-center increment with both DFLCCSD(T) and DF-LMP2. The first nearest-neighbor, two-center increment (with both correlated molecules within the unit cell) was also calculated for DF-LMP2. The improved, extended basis set utilized for these 


\begin{tabular}{lcccc}
\hline \hline $\mathrm{Cl}_{2}$ & & & & \\
\hline Method & DF-LMP2 & DF-LCCSD(T0) & DF-LCCSD(T) & T-T0 \\
\hline 1-center & 2481 & 2848 & 2802 & -46 \\
2-center & -25246 & -21459 & -21632 & -173 \\
3-center & -65 & 809 & $809(\mathrm{~T} 0)$ & - \\
& & & & \\
\hline & & & & \\
Inc. Totals & -22830 & -17801 & & \\
& & & &
\end{tabular}

Lit. $E_{\text {coh }} \quad-12179$

Table 8.6. The one-, two- and three-center incremental energies for solid chlorine computed with DF-LMP2 and DF-LCCSD(T0), compared to the (zero-point corrected) cohesive energy for chlorine [76]. The results of the full-triples evaluation for the density-fitted local coupled cluster calculations of the one- and two-centers are also included, as well as the energy gain from the T0 approximation, illustrating that a full-triples evaluation adds relatively little to the two-center energies justifying its exclusion from the three-center calculations. 


\begin{tabular}{lcccc}
\hline \hline $\begin{array}{l}\mathbf{B r}_{2}, \text { LC ECP } \\
E_{\text {corr }}(\mu \mathbf{H})\end{array}$ & & & & \\
\hline Method & DF-LMP2 & DF-LCCSD(T0) & DF-LCCSD(T) & T-T0 \\
\hline & & & & \\
1-center & 3515 & 4534 & 4353 & -195 \\
2-center & -39794 & -33481 & -33744 & -263 \\
3-center & -33 & 1386 & $1386(\mathrm{~T} 0)$ & - \\
& & & & \\
\hline & & & & \\
Inc. Totals & -36312 & -27561 & & \\
& & & & \\
\hline
\end{tabular}

Table 8.7. The one-, two- and three-center incremental energies for solid bromine (LC model) computed with DF-LMP2 and DF-LCCSD(T0), compared to the (zero-point corrected) cohesive energy for chlorine [76]. The results of the full-triples evaluation for the density-fitted local coupled cluster calculations of the one- and two-centers are also included, as well as the energy gain from the T0 approximation, illustrating that a fulltriples evaluation adds relatively little to the two-center energies justifying its exclusion from the three-center calculations. 


\begin{tabular}{lcccc}
\hline \hline $\mathbf{I}_{2}$ & & & & \\
$E_{\text {corr }}(\mu \mathbf{H})$ & & & & \\
\hline Method & DF-LMP2 & DF-LCCSD(T0) & DF-LCCSD(T) & T-T0 \\
\hline & & & 7182 & -185 \\
1-center & 5681 & 7367 & -42957 & -308 \\
2-center & -51881 & -42649 & $2212(\mathrm{~T} 0)$ & - \\
3-center & -100 & 2212 & & \\
& & & & \\
\hline & & & & \\
Inc. Totals & -46300 & -33070 & & \\
& & & & \\
\end{tabular}

Lit. $E_{\text {coh }} \quad-24221$

Table 8.8. The one-, two- and three-center incremental energies for solid iodine computed with DF-LMP2 and DF-LCCSD(T0), compared to the (zero-point corrected) cohesive energy for chlorine [76]. The results of the full-triples evaluation for the density-fitted local coupled cluster calculations of the one- and two-centers are also included, as well as the energy gain from the T0 approximation, illustrating that a full-triples evaluation adds relatively little to the two-center energies justifying its exclusion from the three-center calculations. 
calculations (as given in Tabs. C.6, C.7 and C.8) has additional functions compared to the LC basis. As each additional basis set improves the accuracy of the correlation calculation for all electrons, two calculations were required in order to distinguish the effects of the correlated $d$-electrons from those of the enhanced basis for all electrons.

As bromine has an atomic number of 35, the SC model treated the first 10 electrons ([Ne]) as core, leaving 25 correlated valence electrons per atom. Using the one-center increment as an example, the first step in determining the $d$-correlation energy contribution calculates the energy for the correlated molecule using the small effective core potential (ECP) of [Ne] and correlating all 50 valence electrons. The second step uses the same small ECP, but correlates only 14 valence electrons (i.e., the same number as the LC model). In subtracting the correlation energy of the second step from the first, the correlation contribution of the $d$-electrons is differentiated from that of the improved basis set.

Tab. 8.9 gives the results of the one-center incremental calculation for both DF-LCCSD(T) and DF-LMP2. It is interesting to note that the relative energy contributions from $d$-correlation is quite different between the two methods, representing 25\% for DF-LMP2 and only 15\% for DF-LCCSD(T) for the first two-center incremental calculations for the $\mathrm{SC} \mathrm{Br}_{2}$ model using DF-LMP2. The LC incremental energies for bromine are given for comparison, as well as the relative percentage of the $d$-correlation energy to the LC energy. In order to estimate the overall effect of the $d$-electrons, the $d$-correlation percentage of the first 2-center (9.2\%) is used to extrapolate estimates for the total $d$-correlation contributions from the two- and three-centers. As illustrated in the table, the $d$-correlation energy for solid bromine would contribute $\sim 7.2 \%$ of the overall binding energy, if these percentages remained accurate for the remainder of the calculations. 


\begin{tabular}{|c|c|c|c|c|c|c|}
\hline \multicolumn{7}{|l|}{$\mathbf{B r}_{2}$} \\
\hline \multirow[t]{2}{*}{$E_{\text {corr }}(\mu \mathbf{H})$} & \multicolumn{5}{|c|}{ DF-LMP2 } & \multirow[b]{2}{*}{$d_{\text {corr }} / \mathbf{L C} \%$} \\
\hline & LC & $\mathbf{S C}_{7 v e}$ & $\mathbf{S C}_{25 v e}$ & $d_{\text {corr }}$ & $\mathbf{L C}+d_{\text {corr }}$ & \\
\hline 1-center & 3515 & 3811 & 4700 & 889 & 4404 & $25.3 \%$ \\
\hline first 2-center & -8066 & -7956 & -8699 & -743 & -8808 & $9.2 \%$ \\
\hline 1-center & 3515 & 3811 & 4700 & 889 & 4404 & $25.3 \%$ \\
\hline 2-center & -39794 & - & - & $-3664^{*}$ & $-43458^{*}$ & $9.2 \% *$ \\
\hline 3-center & -33 & - & - & $-3^{*}$ & $-36^{*}$ & $9.2 \% *$ \\
\hline Totals & -36312 & - & - & $-2778^{*}$ & $-39090^{*}$ & $7.2 \% *$ \\
\hline \multicolumn{7}{|c|}{ DF-LCCSD(T) } \\
\hline 1-center & 4354 & 4642 & 5297 & 655 & 5009 & $15 \%$ \\
\hline
\end{tabular}

Table 8.9. The one-center $d$-correlation energy contributions for both DFLMP2 and DF-LCCSD(T). The first two-center $d$-correlation is also included for DF-LMP2, with total values extrapolated to yield estimates (as indicated by italicized values ${ }^{*}$ ) of the total $d$-correlation contribution to the two- and three-center incremental energies, as well as the overall incremental total. 


\begin{tabular}{lc}
\hline \hline $\begin{array}{l}\text { Zero-Point Correction to } E_{c o h} \\
\mathbf{C l}_{2}\end{array}$ \\
\hline Functional & $E_{c o h}(\mu \mathbf{H})$ \\
\hline B3LYP & 923 \\
PBE & 702 \\
PBE0 & 923 \\
PWGGA & 695 \\
Lit. A & 633 \\
Lit. $B$ & 712 \\
\hline \hline
\end{tabular}

Table 8.10. The zero-point energies for chlorine, calculated using a variety of DFT functionals as well as two reference values: Lit. A from Ref. [76] and Lit. B from Ref. [195].

\subsection{Zero-point energies}

Zero point energies represent the ground state, or lowest possible, energy at $0 \mathrm{~K}$ due to a quantum mechanical system's wave-like nature. Although the literature value obtained for the cohesive energies of $\mathrm{Cl}_{2}, \mathrm{Br}_{2}$ and $\mathrm{I}_{2}$ had included zero-point corrections [76], the DFT approximation of the zero-point energy for solid chlorine was calculated for reference. These energies are given in Tab. 8.10 for a variety of functionals, along with two values from literature for comparison.

\subsection{Bulk Hartree-Fock Calculations}

Although MI allows for the calculation of electron-electron correlation in large systems at the post-HF level of accuracy, the ultimate goal is an accurate calculation of the total cohesive energy of the solid. For MI calculations, this total cohesive energy is given by Eq. (2.56) and involves the 
sum of the bulk HF energy, $E_{\mathrm{HF}}$, which accounts for all energies excluding correlation, and the additive correlation energy, $E_{\text {corr }}$, which accounts for this excluded energy. The previous sections outlined how the additive correlation energy is calculated by MI, while the methods for calculating the HF bulk energy will be addressed here.

Bulk calculations were completed using a combination of CRYSTAL and CRYSCOR. In general overview, CRYSTAL is run to obtain a HF energy for the bulk solid, giving an estimate to the total cohesive energy of the solid lacking only the correlation contribution. A CRYSCOR localization is completed to recast the problem in terms of localized orbitals, which are then used to calculate the bulk MP2 correlation energy. This MP2 calculation yields a post-HF correlation estimate for the additive correlation energy of bulk crystal, along with a Lennard-Jones (LJ) estimate for the long-range correlation contributions to the unit cell cohesive energy. This LJ energy is obtained by extrapolating the MP2 correlation energy to large distances from the unit cell.

A counterpoise calculation is completed for both the HF and MP2 energies, using clusters of ghost atoms surrounding the unit cell at various radii. These counterpoise values are subtracted from the HF and MP2 energies to yield estimates of the bulk cohesive energy at the HF and MP2 level at a series of counterpoise radii. Finally, the series of HF and MP2 cohesive energies as a function of counterpoise radius can be fit using a polynomial or exponential curve, to give an estimate of the extrapolated $\mathrm{HF}$ and MP2 total cohesive energies of the solid.

\subsubsection{Dual Basis Set Scheme}

While these bulk calculations will yield a relatively accurate estimate of the bulk cohesive energy of the solid at two levels of theory (HF and MP2), a problem arises based on the influence of basis set selection for each code. A large, diffuse basis set is required for accurate, post-HF calculations in 
CRYSCOR; however, this diffuse basis set causes convergence problems within CRYSTAL. This problem is addressed by using a dual basis set scheme for the bulk calculations.

This scheme begins with a large, diffuse basis set, from which the diffuse functions are incrementally eliminated until the initial HF calculation converges. This reduced basis set is used to run the localization in CRYSCOR. However, before the MP2 calculation is completed, a second HF calculation is completed in CRYSTAL using the full, diffuse basis set coupled with the 'GUESDUAL' keyword. This keyword forces Crystal to construct a density matrix for the new basis set, using the results of the previous calculation as an initial guess, but stops the SCF calculation after the first iteration. The output from this calculation is then used for the subsequent MP2 calculation in CRYSCOR, which uses the full diffuse basis set and the keyword 'DUALBAS' to signal the dual basis set scheme. In addition to the MP2 correlation energy, this calculation yields the "singlesenergy" representing the amount of energy gained by the HF calculation from that single SCF iteration with the improved basis set. Although not a converged value, the singles-energy represents an improvement to the HF calculation and is subsequently used as a correction to the HF bulk cohesive energy. The most accurate estimate for the HF bulk cohesive energy will be calculated when the difference between the two basis sets is minimal, and the singles-energy is small in comparison to the HF energy.

Bulk calculations employing the dual basis set scheme have been completed for solid chlorine. The reference basis set was identical to that used for DFT calculations, as outlined in Sec. 8.1. This reference basis set was modified to from the large, diffuse basis by adding one s-, $\mathrm{p}$ - and $\mathrm{d}-$ function as well as $2 \mathrm{f}$-functions. These small and large basis sets are given in Tabs. C.1 and C.2 respectively. Overall, the dual basis set scheme allows for the calculation of both the HF bulk cohesive energy as well as the postHF (MP2) additive correlation contribution to the cohesive energy. The MP2-estimate of the total cohesive energy can be used for further com- 
parison with both experimental values as well as the MI-determined total cohesive energy, as described in Eq. (2.56). The HF bulk cohesive energy (to include the singles-energy correction) gives the value for $E_{\mathrm{HF}}$ within that equation while the MI incremental calculations, as outlined in the following section, describe the method for obtaining the additive correlation contribution, $E_{\text {corr }}$.

\subsubsection{Bulk $\mathrm{Cl}_{2}$ results}

The results of these bulk calculations are given in the upper portion of Tab. 8.11. The second column lists the cohesive energy of the periodic HF calculation, completed with the reduced basis set and counter-poise corrected up to a radius as listed in the first column. The third column listing the singles energy gives the additional correction to the HF cohesive energy from the single iteration with the improved basis set, also corrected for BSSE. The fourth column lists the long-range Lennard-Jones extrapolated contribution, counterpoise corrected for BSSE, with the fifth being the totals from each of these contributions to give an estimate of the cohesive energy of solid chlorine as calculated via the dual basis set scheme. The final row of this table gives an extrapolated value for each of the columns, as if one had performed BSSE corrections to an infinite radius, which has been obtained by a simple polynomial fit to the previous data. It should be noted that the bulk HF cohesive energy is repulsive, indicating that in the absence of correlation contributions, solid chlorine would be non-binding. This result is somewhat intuitive for a van der Waals bound system solid such as chlorine, although this same correlation-dominated binding was previously noted for metallic solid mercury [187, 111, 112].

The bottom portion of Tab. 8.11 illustrates the cohesive energies for chlorine, with the correlation energies obtained by the incremental scheme (MI) instead of the LJ-extrapolation. Taking the HF cohesive energy and adding the singles contribution yields an estimate of the cohesive energy 


\begin{tabular}{crrrr}
\hline \hline $\mathbf{C l}_{2}(\mu \mathbf{H})$ & & & & \\
\hline$r_{B S S E}(\AA)$ & $E_{c o h}(\mathbf{H F})$ & singles & LJ extrap. & Total \\
\hline 0.0 (isolated) & -11507 & +12842 & -21064 & -19729 \\
4.0 & +1762 & +4583 & -19729 & -13384 \\
5.0 & +7040 & +1487 & -19332 & -10805 \\
7.0 & +7675 & +1069 & -19280 & -10536 \\
extrapolated & +8218 & +712 & -19236 & -10306
\end{tabular}

\begin{tabular}{crrr}
\hline \hline & & \\
& & & \\
$E_{\text {coh }}(\mathbf{H F})+$ DF-LMP2 & DF-LCCSD(T) \\
1-center & (from above) & +8930 & +8930 \\
2-center & +2481 & +2802 \\
3-center & -25246 & -21632 \\
& -64 & +809 \\
\hline & & \\
Inc. Totals & & -9091 \\
& -13900 & \\
\hline
\end{tabular}

Lit. $E_{c o h}$

$-12179$

Table 8.11. The results of the dual basis set bulk calculations for the cohesive energy of chlorine. Adding in the one-, two- and three-center method of increments results from Tab. 8.6, and comparing these results to the literature-referenced cohesive energy for chlorine [76]. 
not accounting for correlation. Using MI to account for this additive correlation contribution, one obtains the results listed in the second to last row of the table. DF-LMP2 significantly overestimates the cohesive energy while DF-LCCSD(T0) significantly underestimates the cohesive energy, as compared to the literature value [76]. It was expected that the DF-LCCSD(T0) results would give a closer estimate of the cohesive energy. As the source of the reference cohesive energy is unclear (referenced in [76] as a private communication and likely derived from the heat of sublimation), it is possible that this is simply a poor estimate of the true cohesive energy of chlorine. As a final point, it is interesting to note that solid chlorine would be unbound in the absence of 2-center correlations, which contribute all of the binding energy for the DF-LCCSD(T) method and $99.6 \%$ of the binding energy for DF-LMP2.

\subsection{Summary}

In summary, calculations revealed that DFT cohesive energies strongly depend on the functional employed, and vary greatly between each functional. Tab. 8.12 gives a summary of the zero-point corrected DFT cohesive energies for each functional as well as the percent deviation from the literature value, given by Ref. [76]. As expected, the dispersion corrected values yield more accurate cohesive energies than their non-dispersion corrected counterparts. $\mathrm{PBE}+\mathrm{d}$ calculates yields the closest approximation to the literature value of cohesive energy.

Method of increments calculations were completed for one-, two- and three-center increments of chlorine, bromine and iodine for the LC model. For each solid, the one-center increment is repulsive while the two-center incremental energy is large and attractive. The three-center increments were small and attractive for DF-LMP2, but repulsive for DF-LCCSD(T). The two-center increment contributed the largest correlation contribution to binding for each solid halogen. 


\begin{tabular}{lrc}
\hline \hline Functional & $E_{c o h}$ & Lit. $-E_{c o h} /$ Lit. $\%$ \\
\hline B3LYP & 1825 & $114 \%$ \\
B3LYP+d & -8356 & $31 \%$ \\
B3LYP+d* & -7023 & $42 \%$ \\
PBE & -2405 & $80 \%$ \\
PBE+d & -9192 & $26 \%$ \\
PBE0 & -495 & $96 \%$ \\
PWGGA & -4008 & $67 \%$ \\
\hline Lit. & -12179 & \\
\hline \hline
\end{tabular}

Table 8.12. The zero-point corrected DFT cohesive energies for chlorine, combining the results listed in Tab. 8.1 and 8.10, as well as the percent deviation from the literature value [76].

Total cohesive energy calculations were completed for $\mathrm{Cl}_{2}$, combining the incremental correlation contributions to binding with the bulk HF cohesive energy. Similar to mercury, solid chlorine would be unbound in the absence of two-center correlations. Tab. 8.13 gives the total cohesive energy calculated for both DF-LMP2 and DF-LCCSD(T), as well as the percent deviation from the literature value. As expected, DF-LMP2 overestimates binding while DF-LCCSD(T) underestimates binding. Neither the DF-LMP2 or DF-LCCSD(T) method of increments cohesive energy yields an accurate cohesive energy, though the DF-LCCSD(T) cohesive energy is closer to the literature value.

Comparing the DF-LCCSD(T) cohesive energy to the best DFT cohesive energy (from Tab. 8.12), the DF-LCCSD(T) value is very close to the results obtained by the $\mathrm{PBE}+\mathrm{d}$ functional. It should be noted that, unlike DFT, the method of increments value can be systematically improved through the use of higher-order increments (4-center, 5-center, etc.). Given the already small contributions from the three center increments, however, 


\begin{tabular}{lrc}
\hline \hline Method & $E_{c o h}$ & $\mid$ Lit. $-E_{c o h} /$ Lit. $\mid$ \\
\hline DF-LMP2 & -13900 & $14 \%$ \\
DF-LCCSD(T) & -9091 & $25 \%$ \\
\hline Lit. & -12179 & \\
\hline \hline
\end{tabular}

Table 8.13. The method of increments DF-LMP2 and DF-LCCSD(T) cohesive energies for chlorine, from Tab. 8.11, as well as the percent deviation from the literature value [76].

it is likely that completing a more robust BSSE-extrapolation for the bulk HF cohesive energy and singles-energy, as shown in upper portion of Tab. 8.11, would yield larger improvements to the incremental cohesive energy than higher-order increments. An additional source of error could be attributed to the literature value, as this cohesive energy was derived from the heat of sublimation and may not accurately represent the cohesive energy of molecular solids [196].

The one-center calculations for the $\mathrm{SC} \mathrm{Br}_{2}$ model illustrate that $d$-correlation contributes $\sim 25 \%$ of the one-center correlation energy for DF-LMP2, but only $\sim 15 \%$ for DF-LCCSD(T). The first two-center calculation for DF-LMP2 shows that the relative $d$-correlation contribution is significantly less than for the one-center, contributing only $\sim 9 \%$. Extrapolating this ratio to all two- and three-center incremental yields a total $d$-correlation energy amounting to $\sim 7 \%$ of the total LC incremental energy.

Overall, these calculations illustrate that MI calculations yield an adequate estimate of the total cohesive energies of the solid halogens. Although the DF-LCCSD(T) and DF-LMP2 incremental cohesive energies for chlorine do not accurately calculate the literature value, they can be systematically improved through the use of higher-order increments and improved BSSE-extrapolations for the HF bulk energy. Additionally, the validity of this literature value remains questionable. As has been previously 
noted in the extensive body of theoretical work on modelling the solid halogens, molecular solids represent complicated systems whose properties are not easily accessible by even the most accurate $a b$ initio methods.

In summary to this body of research investigating both cluster and bulk properties of molecular solids, the following chapter offers concluding remarks, highlighting important results for both the small gallium cluster thermodynamic modelling as well as the method of increments calculations. 


\section{Chapter 9}

\section{Conclusions}

\subsection{Gallium Clusters}

FPMD parallel tempering molecular dynamics simulations were completed for clusters ranging from 7-35 atoms, covering two extended size ranges containing both neutral and cationic charge states as well as two pseudopotential models. The results have shown that the parallel tempering (PT) algorithm identified lower energy configurations than the initial structures for many cluster sizes, with only the 34-atom cluster proving to have a lower energy structure not explored by PT. As demonstrated by the PT-induced capped-sphere to stacked-plane transitions for the 20atom cluster, this algorithm aided each simulation in adequately exploring gallium's complex potential energy surface.

Results for the 20-atom cluster clearly established that the pseudopotential treatment of the $d$-electrons did not affect the simulated melting behavior. These simulations also demonstrated that removing just one electron can alter the melting temperature by up to $100 \mathrm{~K}$, proving the experimentally observed single-atom size-sensitivity also extends to singleelectron differences as well. The microcluster simulations set a lower size limit on what can be defined as melting. These simulations additionally illustrated that greater-than-bulk melting is not universal for small gallium 
clusters, with $\mathrm{Ga}_{12}$ exhibiting a melting transition at $178 \mathrm{~K}, \sim 125 \mathrm{~K}$ lower than the bulk melting temperature for gallium. Simulations for $\mathrm{Ga}_{20}^{+}$and $\mathrm{Ga}_{32}^{+}-\mathrm{Ga}_{35}^{+}$demonstrated that FPMD can model the experimentally measured specific heat curves with a fair degree of accuracy, although there is typically a $\sim 75 \mathrm{~K}$ difference between the simulated and experimental $T_{m}$. For all but the 33-atom cation, the simulated melting temperature is lower than experimental measurements.

Summaries of the cohesive energies and simulated melting temperatures for each of the cluster sizes are illustrated in Figs. 9.1 and 9.2, respectively. Plotted versus the cube root of the number of atoms, the cohesive energy trend clearly establishes the dominance of surface effects for bonding in these small clusters. Undoubtedly breaking the general size trend, the relative stability of the 8 - and 33-atom clusters is exceptional. The melting temperatures exhibit no notable size dependency. The 10- and 33-atom clusters have the highest melting temperatures for their respective sizeranges. There is no overall correlation between melting temperature and cohesive energies, although the 33-atom cluster is an outlier in both plots.

Although an unprecedented number of cluster sizes have been simulated, the chemistry underlying gallium cluster greater-than-bulk melting and extreme size-sensitivity could not be conclusively identified. However, two universal results have emerged from these simulations that prove instructive in addressing these thermodynamic anomalies. The first is that no evidence of covalent bonding is identified for any of the 12 simulated clusters. In fact, ELF and SH-PDOS indicate a more metallic nature for each of the clusters, with surface-dominated electron localization and conducting states at the Fermi level. Although results of the Bader analysis demonstrate a non-metallic charge polarity with negative central atoms and positive/neutral surface atoms, this could be due to the significantly higher coordination of these interior atoms causing an enlargement of the Bader volumes for these atoms, thereby non-physically attributing more charge density to these atoms. 


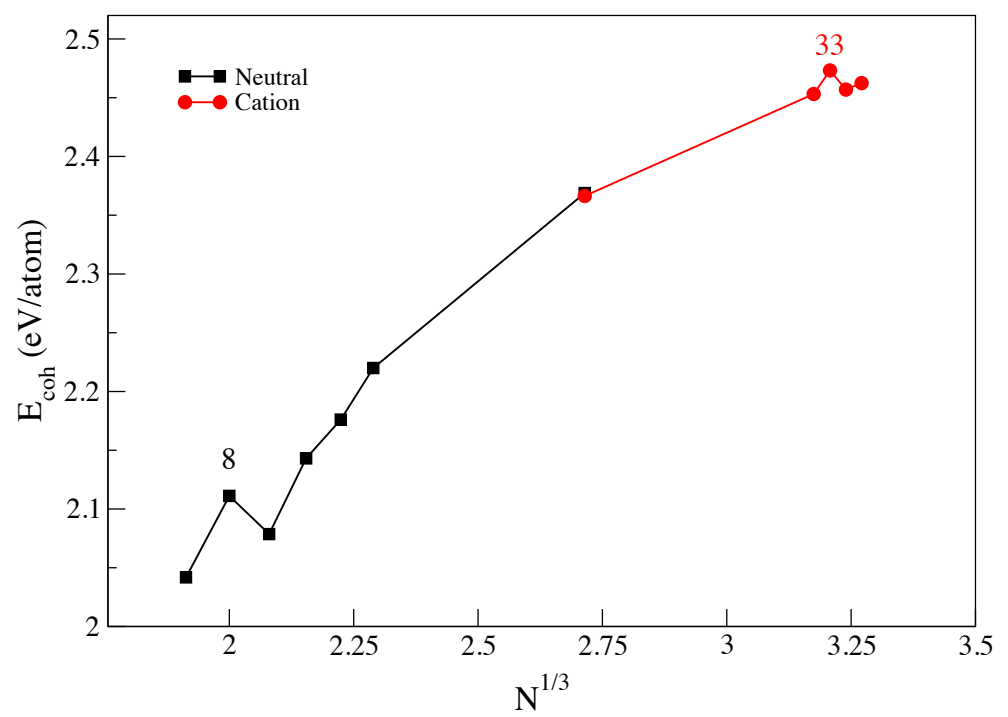

Figure 9.1. A summary of the cohesive energies for the lowest-energy structures of each of the cluster sizes, as determined by the LC model for the neutral clusters (black) and cations (red). Plotted against $N^{1 / 3}$, the cohesive energy trend clearly illustrates the effects of surface-versus-bulk bonding. Breaking this trend are the 8- and 33-atom clusters, with notably higher cohesive energies.

The second universal result is that pair bonding similar to that observed in bulk gallium was only identified for the lowest energy PT-derived 8-atom cluster. All other PT-derived structures exhibit radial distribution functions peaking at $\sim 2.7-2.8 \AA$, similar to the second nearest neighbor distance in bulk gallium. As $\mathrm{Ga}_{8}$ falls below the size-limit where melting can be clearly defined, it is difficult to draw thermodynamic comparisons between this cluster and those lacking bulk-like pair bonding.

Despite this limitation, it is clear that both the non-covalent nature and lack of pair bonding observed for small gallium clusters distinguishes the nature of bonding in these small clusters from that of bulk gallium. While metallicity has not yet been clearly established, these simulations have 


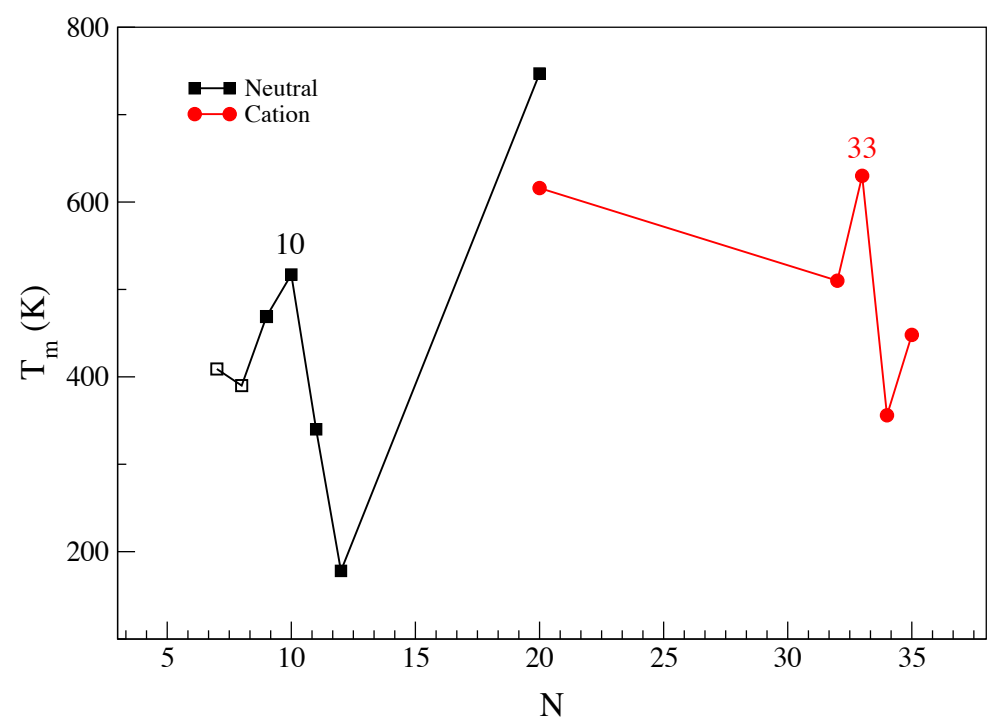

Figure 9.2. A summary of the simulated melting temperatures for the neutral (black) and cationic (red) clusters (LC model). The open squares for the 7- and 8-atom clusters illustrate the value of the peak in the specific heat curve as opposed to a melting temperature. While no overall trends are identified, the 10- and 33-atom clusters have notably higher melting temperatures within their respective size ranges.

yielded clear evidence to support a more metallic nature for the clusters than has been reported for bulk gallium. As true metals typically have higher melting points than covalently bonded solids, increased metallicity would go some way to explaining the greater-than-bulk melting trend observed for these small clusters. The one counterpoint to this argument is the 12-atom simulation. This cluster exhibits a melting transition at 179 $\mathrm{K}$, but demonstrates the same ELF, low density of states at the Fermi level and lack of pair bonding observed for clusters with greater-than-bulk $T_{m}$.

Looking to structural trends in the lowest energy identified structures, however, both this $\mathrm{Ga}_{12}$ exception and the single-atom size sensitivity can be explained by surface instabilities introduced by ad-atoms. This argu- 
ment is most clearly supported by comparison of the results for the 8-/9atom simulation pairs as well as the 33-/34-atom simulation pairs. While the definition of melting breaks down for the 8-atom cluster, it is clear from $\delta_{r m s}$ and MSD measurements that the 8 -atom rhomboid is very strongly bound across all simulated energies. Once a single atom is added to this stable base unit, however, the simulation results change dramatically, exhibiting a melting-like transition at a relatively low $479 \mathrm{~K}$.

A similar change occurs between the 33- and 34-atom simulations. The 34-atom cluster exhibits a simulated melting transition at $379 \mathrm{~K}$, while the 33 -atom cluster nearly doubles that at $630 \mathrm{~K}$. The lowest energy structure identified for the 34-atom configuration, as illustrated in Fig. 7.10, only differs from the lowest-energy 33-atom structure (Fig. 7.8 (a)) by the single ad-atom attached to one of the surfaces. In both the 8-/9- atom and 33-/34-atom cases, it appears that single-atom additions yield lower structural stabilities that manifest in their thermodynamic behaviors. In keeping with this argument and looking to the SH-PDOS of the lowest energy structure identified for the $\mathrm{Ga}_{12}$ simulation, this structure could be viewed as the stable $\mathrm{Ga}_{8}$ rhomboid with four ad-atoms. Although it is not assumed that increased surface destabilization linearly corresponds to increasing numbers of ad-atoms, this argument does offer one possible explanation for $\mathrm{Ga}_{12}$ 's low melting temperature.

As a final note, the tendency for the larger clusters to arrange themselves in planes of atoms is noteworthy and should be mentioned here. One set of experimental data has indicated that bulk gallium's surface is quite metallic, comprised of split-dimer atoms lying in planes perpendicular to the dimer axis [15]. Observed from a symmetric side-orientation, all clusters exhibiting melting temperatures significantly exceeding those of bulk gallium have a triple-plane structure. The central atoms, each having very low MSD's, comprise the middle plane while the surface atoms make up the outer two planes. This cluster structure could be viewed as two metallic planes sandwiching bulk-like atoms. Experimental evidence has 
shown that gallium's bulk surface oriented perpendicular to the dimers remained solid even after the interior atoms had melted [24, 23]. The fact that gallium clusters, exhibiting surface-like structural motifs, also melt at temperatures far exceeding that of bulk gallium seems more than a chance circumstance.

\subsection{Solid Halogens}

Method of increments (MI) calculations were completed for the one-, twoand there-body correlation energies of solid $\mathrm{Cl}_{2}, \mathrm{Br}_{2}$ and $\mathrm{I}_{2}$. As has been previously noted in theoretical studies of the solid halogens, modelling the characteristics of these covalently pair-bonded systems presents a number of challenges. Given the lack of universally accepted experimental cohesive energies for each system, it is difficult to address how accurately the method of increments captures the correlation energy for the solid halogens. Literature citing the cohesive energies for $\mathrm{Cl}_{2}, \mathrm{Br}_{2}$ and $\mathrm{I}_{2}$ derive these values from the heats of sublimation $[70,195,77,76]$, the validity of which has been called into question [196].

It is interesting to note the similarities between solid chlorine and mercury, in the poor description of DFT and two-body correlation energies dictating binding in both systems. As the only two elements in the periodic table exhibiting a liquid structure in their normal temperature, normal pressure phase, bromine and mercury also share at least one commonality. Additionally, solid $\mathrm{Cl}_{2}, \mathrm{Br}_{2}$ and $\mathrm{I}_{2}$ share the same crystal structure as gallium, noting differences in the lattice parameters and ratios for each element. These comparisons bring up the question of metallicity in the solid halogens. X-ray diffraction studies completed for each of the solid halogens under pressure indicate an increasing metallicity with increasing pressure for all solid halogens, even in the absence of a metallic transition observed for iodine [75]. What is the change in the nature of bonding that leads to such a transition? Although metals are more diffi- 
cult to model with the method of increments due to the absence of a band gap, method of increments calculations for both gallium and iodine under pressure would offer additional insights into the nature of metallic and covalent bonding, and how each contributes to the wealth of intriguing properties observed in each of these molecular solids.

\subsection{Molecular Solids}

Exploring size-regimes ranging from bulk to clusters and bonding ranging from covalent to metallic, this research has highlighted the need for more than one perspective in theoretically addressing these interesting molecular solids. Gallium has been described as a "molecular metal," which is a useful construct for understanding and characterizing many of its bulk (as opposed to surface or cluster) properties. However, at the smallest sizes, an understanding of bonding in metallic surfaces becomes integral. A similar issue arises for the covalent solid halogens, as the scaling law for the solid halogens demonstrates increasing metallicity with atomic number. Both studies emphasize that a theoretical treatment of molecular solids would greatly benefit from a more extended view.

While calculations accounting for this broader perspective could advance our understanding of these interesting systems, the challenge of accurately modelling the properties of molecular solids is evidenced in the research presented here. While DFT adequately captures the overall thermodynamic behavior of small gallium clusters, the approximate nature of the calculation also yields a significant shift between the experimental and simulated curves. In solid chlorine, the origin of the notable difference between the literature and MI cohesive energies remains an open question. Both studies clearly illustrate that the approximations making each calculation feasible limit the accuracy of the results. Although computational quantum chemistry has advanced considerably in the 80 years since its conception, the exchange between "exact" and "computable," outlined in 
Dirac's quote given as an opening to this thesis, remains a conflict even today. 
Appendices 



\section{Appendix A}

\section{Multiple-Histogram Mathematics}

\section{A.1 Deriving $S_{j}$}

Taking the partial derivative with respect to entropy $S_{j}$ is relatively straightforward, although in order to avoid confusion, a new set of dummy indices $k, \ell$ will be introduced. Taking the partial derivative of eq. 4.28 with respect to $S_{j}$ :

$$
\frac{\partial}{\partial S_{j}} \sum_{k \ell} n_{k \ell}\left(\frac{S_{\ell}}{k_{B}}+\frac{(3 N-8)}{2} \ln \left(E_{k}-U_{\ell}\right)+A_{k}-\ln n_{k \ell}\right)^{2}=0
$$

or

$$
\sum_{k \ell} n_{k \ell} \frac{\partial\left(\frac{1}{k_{B}} S_{\ell}+\frac{(3 N-8)}{2} \ln \left(E_{k}-U_{\ell}\right)+A_{k}-\ln n_{k \ell}\right)^{2}}{\partial S_{j}}=0 .
$$

The partial is only non-zero where $\ell=j$ or $S_{\ell}=S_{j}$, so

$$
\sum_{k \ell} \delta_{j \ell} n_{k \ell} \frac{\partial\left(\frac{1}{k_{B}} S_{\ell}+\frac{(3 N-8)}{2} \ln \left(E_{k}-U_{\ell}\right)+A_{k}-\ln n_{k \ell}\right)^{2}}{\partial S_{j}}=0
$$


or

$$
\sum_{k} n_{k j} \frac{\partial\left(\frac{1}{k_{B}} S_{j}+\frac{(3 N-8)}{2} \ln \left(E_{k}-U_{j}\right)+A_{k}-\ln n_{k j}\right)^{2}}{\partial S_{j}}=0 .
$$

Now evaluating the derivative, this becomes

$$
2 \sum_{k} n_{k j}\left(\frac{1}{k_{B}} S_{j}+\frac{(3 N-8)}{2} \ln \left(E_{k}-U_{j}\right)+A_{k}-\ln n_{k j}\right)=0 .
$$

Multiplying through by $k_{B} / 2$ and expanding ...

$S_{j} \sum_{k} n_{k j}+k_{B} \sum_{k} n_{k j} \frac{(3 N-8)}{2} \ln \left(E_{k}-U_{j}\right)+k_{B} \sum_{k} n_{k j} A_{k}-k_{B} \sum_{k} n_{k j} \ln n_{k j}=0$.

Finally, solving for $S_{j}$, we obtain

$$
S_{j}=\frac{\sum_{k} n_{k j}\left(\ln n_{k j}-\frac{(3 N-8)}{2} \ln \left(E_{k}-U_{j}\right)\right)-\sum_{k} n_{k j} A_{k}}{\sum_{k} n_{k j}} k_{B},
$$

or divided into separate terms as

$$
S_{j}=\frac{\sum_{k} n_{k j} \ln n_{k j}-\frac{(3 N-8)}{2} \sum_{k} n_{k j} \ln \left(E_{k}-U_{j}\right)-\sum_{k} n_{k j} A_{k}}{\sum_{k} n_{k j}} k_{B} .
$$

For this equation, the $\mathrm{k}$ index represents the different energies for the simulations and the $\mathrm{j}$ index represents the configurational energy bins. Rewriting this in terms of the original $i$ and $j$ indices, this becomes

$$
S_{j}=\frac{\sum_{i} n_{i j} \ln n_{i j}-\frac{(3 N-8)}{2} \sum_{i} n_{i j} \ln \left(E_{i}-U_{j}\right)-\sum_{i} n_{i j} A_{i}}{\sum_{i} n_{i j}} k_{B}
$$


where $E_{i}$ represent the 19 different constant energies at which the microcanonical simulations were run and $U_{j}$ are the potential energy bins.

\section{A.2 Deriving $\mathrm{A}_{\mathrm{i}}$}

The derivative for the additive constant, $A_{i}$, is far more complicated. Starting again with Eq. 4.28 and taking the partial derivative with respect to $\mathrm{A}_{\mathrm{i}}$, this equation becomes

$$
\frac{\partial}{\partial A_{i}} \sum_{k \ell} n_{k \ell}\left(\frac{S_{\ell}}{k_{B}}+\frac{(3 N-8)}{2} \ln \left(E_{k}-U_{\ell}\right)+A_{k}-\ln n_{k \ell}\right)^{2}=0
$$

or

$$
\sum_{k \ell} n_{k \ell} \frac{\partial\left(\frac{1}{k_{B}} S_{\ell}+\frac{(3 N-8)}{2} \ln \left(E_{k}-U_{\ell}\right)+A_{k}-\ln n_{k \ell}\right)^{2}}{\partial A_{i}}=0 .
$$

Only terms with $k=i$ or $A_{k}=A_{i}$ will be non-zero in the derivative so eq. A.11 becomes

$$
\sum_{k \ell} \delta_{i k} n_{k \ell} \frac{\partial\left(\frac{1}{k_{B}} S_{\ell}+\frac{(3 N-8)}{2} \ln \left(E_{k}-U_{\ell}\right)+A_{k}-\ln n_{k \ell}\right)^{2}}{\partial A_{i}}=0,
$$

or

$$
\sum_{\ell} n_{i \ell} \frac{\partial\left(\frac{1}{k_{B}} S_{\ell}+\frac{(3 N-8)}{2} \ln \left(E_{i}-U_{\ell}\right)+A_{i}-\ln n_{i \ell}\right)^{2}}{\partial A_{i}}=0 .
$$

Evaluating the derivative with respect to $A_{i}$, this becomes

$$
2 \sum_{\ell} n_{i \ell}\left(\frac{1}{k_{B}} S_{\ell}+\frac{(3 N-8)}{2} \ln \left(E_{i}-U_{\ell}\right)+A_{i}-\ln n_{i \ell}\right)=0
$$


which, multiplying by $1 / 2$ and expanding, is

$$
A_{i} \sum_{\ell} n_{i \ell}+\frac{(3 N-8)}{2} \sum_{\ell} n_{i \ell} \ln \left(E_{i}-U_{\ell}\right)-\sum_{\ell} n_{i \ell} \ln n_{i \ell}+\frac{1}{k_{B}} \sum_{\ell} n_{i \ell} S_{\ell}=0 .
$$

Now this equation is in terms of the two unknowns, but the expression for the entropy in terms of $A_{i}$ is given. Substituting Eq. A.9 into this equation, and setting $j=\ell$ to match indices, yields

$$
\begin{aligned}
A_{i} \sum_{\ell} n_{i \ell}+ & \frac{(3 N-8)}{2} \sum_{\ell} n_{i \ell} \ln \left(E_{i}-U_{\ell}\right)-\sum_{\ell} n_{i \ell} \ln n_{i \ell} \\
& +\frac{1}{k_{B}} \sum_{\ell} n_{i \ell} \frac{\sum_{k} n_{k \ell}\left(\ln n_{k \ell}-\frac{(3 N-8)}{2} \ln \left(E_{k}-U_{\ell}\right)\right)-\sum_{k} n_{k \ell} A_{k}}{\sum_{k} n_{k \ell}} k_{B}=0 .
\end{aligned}
$$

Separating all terms involving $A$, this becomes

$$
\begin{aligned}
A_{i} \sum_{\ell} n_{i \ell}-\sum_{\ell} n_{i \ell} \frac{\sum_{k} n_{k \ell} A_{k}}{\sum_{k} n_{k \ell}} & =\sum_{\ell} n_{i \ell} \ln n_{i \ell}-\frac{(3 N-8)}{2} \sum_{\ell} n_{i \ell} \ln \left(E_{i}-U_{\ell}\right) \\
& -\sum_{\ell} n_{i \ell} \frac{\sum_{k} n_{k \ell}\left(\ln n_{k \ell}-\frac{(3 N-8)}{2} \ln \left(E_{k}-U_{\ell}\right)\right)}{\sum_{k} n_{k \ell}}
\end{aligned}
$$

Terms on the right hand side are knowns, so set them equal to a vector of constants, $C_{i}$, given as

$$
\begin{aligned}
C_{i}= & \sum_{\ell} n_{i \ell} \ln n_{i \ell}-\frac{(3 N-8)}{2} \sum_{\ell} n_{i \ell} \ln \left(E_{i}-U_{\ell}\right) \\
& -\sum_{\ell} n_{i \ell} \frac{\sum_{k} n_{k \ell}\left(\ln n_{k \ell}-\frac{(3 N-8)}{2} \ln \left(E_{k}-U_{\ell}\right)\right)}{\sum_{k} n_{k \ell}}
\end{aligned}
$$


or divided into separate terms

$$
\begin{aligned}
C_{i}= & \sum_{\ell} n_{i \ell} \ln n_{i \ell}-\frac{(3 N-8)}{2} \sum_{\ell} n_{i \ell} \ln \left(E_{i}-U_{\ell}\right) \\
& -\sum_{\ell} n_{i \ell} \frac{\sum_{k} n_{k \ell} \ln n_{k \ell}-\frac{(3 N-8)}{2} \sum_{k} n_{k \ell} \ln \left(E_{k}-U_{\ell}\right)}{\sum_{k} n_{k \ell}} .
\end{aligned}
$$

Looking at the left hand side of Eq. A.17, the first term involving $A_{i}$ can be rewritten in terms of $A_{k}$ as

$$
A_{i} \sum_{\ell} n_{i \ell}=\sum_{k \ell} n_{i \ell} A_{k} \delta_{k i}
$$

The second term can be rewritten so that the numerator k-sum can be combined with the first $\ell$-sum, because the $n_{i \ell}$ doesn't depend on $\mathrm{k}$. However, it would affect the denominator which does depend on $k$, so a new index ' $\mathrm{m}$ ' is introduced, giving

$$
\sum_{k \ell} n_{i \ell} \frac{n_{k \ell} A_{k}}{\sum_{m} n_{m \ell}} .
$$

Then, the left hand side can be rewritten entirely in terms of $A_{k}$ :

$$
\sum_{k \ell} n_{i \ell} A_{k} \delta k i-\sum_{k \ell} n_{i \ell} \frac{n_{k \ell} A_{k}}{\sum_{m} n_{m \ell}} .
$$

Factoring common terms and remembering the right hand side yields

$$
\sum_{k \ell} n_{i \ell}\left(\delta k i-\frac{n_{k \ell}}{\sum_{m} n_{m \ell}}\right) A_{k}=C_{i} .
$$

Defining $D_{k i}$ to be the $A_{k}$ prefix, as

$$
D_{k i}=\sum_{\ell} n_{i \ell}\left(\delta k i-\frac{n_{k \ell}}{\sum_{m} n_{m \ell}}\right) \text {, }
$$


Eq. A.23 can be rewritten as

$$
\sum_{k} D_{k i} A_{k}=C_{i}
$$

Both the $k$ and $i$ index represent the number of constant energy subruns, which is 19 for these simulations. Therefore, this final equation defines $D_{k i}$ to be a matrix of dimension $19 \times 19$ and $C_{i}$ is a vector of length 19 . This system of equations is simply the linear algebra equation

$$
\mathbf{D} A=\mathbf{C}
$$

which can be solved as

$$
A=\mathbf{D}^{-1} \mathbf{C}
$$

Remembering that the $k$ and $i$ indices are interchangeable, the $A_{k}$ given here represents the original $A_{i}$ from Eq. 4.28. It should be noted that Eq. (A.27) is degenerate, so only the relationship between each respective $A_{i}$ is meaningful. Once the additive constants, $A_{i}$, are known, the configurational entropies can be easily determined from Eq. (A.9).

At every $E_{i}, n_{i j}$ forms a histogram that is approximately bell-shaped across the range of ' $j$ ' potential energies. If the constant total energies are selected to be appropriately spaced, these histograms will overlap, as demonstrated in Figure 4.5, allowing the configurational density of states (which is independent of our choice of $E_{i}$ ) to be related between adjacent energy sub-runs through the additive constants, $A_{i}$. This defines $\Omega_{C}$ continuously across the entire total energy range of the simulation. The microcanonical total density of states, $\Omega(E)$, can be obtained by solving Eq. 4.17 for $\Omega(E)$ and integrating across the energy range as

$$
\Omega(E)=\int_{0}^{E} \Omega_{K}(K) \Omega_{C}(E-K) \mathrm{d} K .
$$




\section{Appendix B}

\section{Atomic Coordinates}

\begin{tabular}{lrrr}
\hline $\begin{array}{l}\text { 6 Ga } \\
\text { xyz }\end{array}$ & & & \\
$\mathrm{Ga}$ & -0.928669 & -0.581092 & 2.189646 \\
$\mathrm{Ga}$ & -0.928669 & -0.581092 & -2.189646 \\
$\mathrm{Ga}$ & 0.543613 & 0.942662 & 0.000000 \\
$\mathrm{Ga}$ & -1.979266 & 0.421417 & 0.000000 \\
$\mathrm{Ga}$ & 1.646495 & -0.100948 & 2.164556 \\
$\mathrm{Ga}$ & 1.646495 & -0.100948 & -2.164556 \\
\hline
\end{tabular}

Table B.1. Atomic coordinates for the lowest energy $\mathrm{Ga}_{6}$ structure. 


\begin{tabular}{lrrr}
\hline $32 \mathrm{Al}$ & & & \\
$\mathrm{xyz}$ & & & \\
$\mathrm{Al}$ & -0.095554 & -3.490553 & 2.077180 \\
$\mathrm{Al}$ & -0.269687 & -0.394431 & 4.147125 \\
$\mathrm{Al}$ & -1.874868 & -1.996279 & -4.568644 \\
$\mathrm{Al}$ & -2.177573 & 0.347992 & -2.953900 \\
$\mathrm{Al}$ & 2.193934 & 2.758890 & -1.241404 \\
$\mathrm{Al}$ & -0.260484 & 4.201900 & 1.654757 \\
$\mathrm{Al}$ & 0.221540 & -3.029852 & -3.094297 \\
$\mathrm{Al}$ & 2.377539 & -1.877999 & -4.339672 \\
$\mathrm{Al}$ & -0.208878 & 4.175682 & -1.220992 \\
$\mathrm{Al}$ & -2.469904 & 0.323055 & 2.533102 \\
$\mathrm{Al}$ & 2.189067 & -2.181653 & 1.044081 \\
$\mathrm{Al}$ & -2.402020 & -2.416331 & 0.750686 \\
$\mathrm{Al}$ & 2.057060 & 0.301177 & 2.725834 \\
$\mathrm{Al}$ & 0.188791 & -0.229643 & -4.237974 \\
$\mathrm{Al}$ & 0.063985 & 2.034797 & -2.847622 \\
$\mathrm{Al}$ & 2.106127 & 2.681648 & 1.556341 \\
$\mathrm{Al}$ & -2.249119 & -2.181409 & -1.867971 \\
$\mathrm{Al}$ & 2.385691 & -2.014026 & -1.582001 \\
$\mathrm{Al}$ & -2.439838 & 2.665660 & 1.245680 \\
$\mathrm{Al}$ & 0.030523 & -3.293625 & -0.510824 \\
$\mathrm{Al}$ & -0.230555 & 1.974724 & 3.042940 \\
$\mathrm{Al}$ & 2.381840 & 0.473146 & -2.705994 \\
$\mathrm{Al}$ & -2.316846 & 2.596042 & -1.482489 \\
$\mathrm{Al}$ & -2.313690 & 0.159011 & -0.197961 \\
$\mathrm{Al}$ & 2.200729 & 0.345178 & 0.017080 \\
$\mathrm{Al}$ & -0.115402 & 1.750183 & 0.132205 \\
$\mathrm{Al}$ & 0.027710 & -0.594020 & -1.471229 \\
$\mathrm{Al}$ & -0.173511 & -0.769080 & 1.377749 \\
$\mathrm{Al}$ & 1.801518 & -2.175158 & 3.630378 \\
$\mathrm{Al}$ & -2.241987 & -2.181398 & 3.332042 \\
$\mathrm{Al}$ & -0.307807 & -3.119124 & 4.787942 \\
$\mathrm{Al}$ & 1.921666 & 5.155494 & 0.267851 \\
\hline & & & \\
\hline
\end{tabular}

Table B.2. Atomic coordinates for the $\mathrm{Al}_{32}$ structure, obtained from Ref. [129]. 


\begin{tabular}{lrrr}
\hline $32 \mathrm{Ga}$ & & & \\
xyz & & & \\
$\mathrm{Ga}$ & -0.121589 & -3.534905 & 2.037645 \\
$\mathrm{Ga}$ & -0.266919 & -0.376387 & 4.189168 \\
$\mathrm{Ga}$ & -1.830344 & -2.004835 & -4.573345 \\
$\mathrm{Ga}$ & -2.221595 & 0.397200 & -3.015889 \\
$\mathrm{Ga}$ & 2.246588 & 2.801035 & -1.284796 \\
$\mathrm{Ga}$ & -0.304317 & 4.237813 & 1.763583 \\
$\mathrm{Ga}$ & 0.212386 & -3.170625 & -3.071931 \\
$\mathrm{Ga}$ & 2.333647 & -1.935875 & -4.349410 \\
$\mathrm{Ga}$ & -0.257819 & 4.219812 & -1.215283 \\
$\mathrm{Ga}$ & -2.496699 & 0.398879 & 2.518948 \\
$\mathrm{Ga}$ & 2.268235 & -2.229674 & 0.999578 \\
$\mathrm{Ga}$ & -2.433691 & -2.419918 & 0.725726 \\
$\mathrm{Ga}$ & 2.113839 & 0.348851 & 2.790434 \\
$\mathrm{Ga}$ & 0.198721 & -0.216288 & -4.224588 \\
$\mathrm{Ga}$ & 0.055103 & 2.060633 & -2.852460 \\
$\mathrm{Ga}$ & 2.172387 & 2.702501 & 1.577578 \\
$\mathrm{Ga}$ & -2.319825 & -2.190801 & -1.895323 \\
$\mathrm{Ga}$ & 2.498689 & -2.067788 & -1.608945 \\
$\mathrm{Ga}$ & -2.565321 & 2.769104 & 1.219806 \\
$\mathrm{Ga}$ & 0.051852 & -3.476208 & -0.510295 \\
$\mathrm{Ga}$ & -0.238793 & 1.995991 & 3.164123 \\
$\mathrm{Ga}$ & 2.450529 & 0.492939 & -2.778092 \\
$\mathrm{Ga}$ & -2.412263 & 2.688034 & -1.503583 \\
$\mathrm{Ga}$ & -2.225619 & 0.177631 & -0.209999 \\
$\mathrm{Ga}$ & 2.149049 & 0.346336 & 0.000105 \\
$\mathrm{Ga}$ & -0.113295 & 1.815334 & 0.195169 \\
$\mathrm{Ga}$ & 0.054247 & -0.712423 & -1.518575 \\
$\mathrm{Ga}$ & -0.137640 & -0.828466 & 1.469704 \\
$\mathrm{Ga}$ & 1.960139 & -2.198033 & 3.638790 \\
$\mathrm{Ga}$ & -2.408939 & -2.159357 & 3.355702 \\
$\mathrm{Ga}$ & -0.296347 & -3.076758 & 4.692944 \\
$\mathrm{Ga}$ & 1.885603 & 5.146247 & 0.273512 \\
\hline & & &
\end{tabular}

Table B.3. Atomic coordinates for the $\mathrm{Ga}_{32}$ structure, optimized from the $A l_{32}$ structure, as listed in Tab. B.2, using the SC PAW for gallium. 


\begin{tabular}{lrrr}
\hline 33 Al & & & \\
xyz & & & \\
$\mathrm{Al}$ & 0.793736 & 5.220321 & 1.271509 \\
$\mathrm{Al}$ & 2.165068 & 2.993257 & 0.919483 \\
$\mathrm{Al}$ & 1.663069 & -0.449291 & -2.867446 \\
$\mathrm{Al}$ & 4.195859 & 0.050324 & -1.856388 \\
$\mathrm{Al}$ & 0.484629 & 3.821310 & -1.181371 \\
$\mathrm{Al}$ & 2.295421 & 1.811599 & -1.471567 \\
$\mathrm{Al}$ & -0.046255 & 1.789532 & -2.828294 \\
$\mathrm{Al}$ & 3.870154 & 0.899139 & 0.763628 \\
$\mathrm{Al}$ & 4.795163 & -1.768418 & 0.154414 \\
$\mathrm{Al}$ & 3.275506 & -2.489702 & -2.001626 \\
$\mathrm{Al}$ & 2.375787 & -3.248888 & 0.573736 \\
$\mathrm{Al}$ & -2.666513 & 1.072662 & -3.326716 \\
$\mathrm{Al}$ & -2.886410 & -3.673463 & 1.234683 \\
$\mathrm{Al}$ & -2.223602 & 2.876033 & -1.393010 \\
$\mathrm{Al}$ & 0.693728 & -2.765198 & -1.648456 \\
$\mathrm{Al}$ & -1.893223 & -3.147518 & -1.389588 \\
$\mathrm{Al}$ & -0.239024 & -3.785457 & 0.767429 \\
$\mathrm{Al}$ & -3.505700 & -1.312611 & -2.666498 \\
$\mathrm{Al}$ & -0.926720 & -0.641922 & -2.036611 \\
$\mathrm{Al}$ & -0.259795 & 1.284392 & -0.030364 \\
$\mathrm{Al}$ & -1.255820 & -1.234079 & 0.543513 \\
$\mathrm{Al}$ & 1.872757 & -0.703230 & -0.144322 \\
$\mathrm{Al}$ & -1.600894 & 4.431966 & 0.616678 \\
$\mathrm{Al}$ & -4.199903 & -1.915714 & -0.176573 \\
$\mathrm{Al}$ & -3.072974 & 0.473857 & -0.623953 \\
$\mathrm{Al}$ & 1.517966 & 0.725703 & 2.225696 \\
$\mathrm{Al}$ & 0.734873 & -1.820435 & 2.518876 \\
$\mathrm{Al}$ & -0.189601 & 2.836015 & 2.354969 \\
$\mathrm{Al}$ & 3.348345 & -1.320488 & 2.364520 \\
$\mathrm{Al}$ & -1.046453 & 0.386852 & 2.833151 \\
$\mathrm{Al}$ & -2.669493 & 2.127052 & 1.399382 \\
$\mathrm{Al}$ & -1.822392 & -2.150875 & 3.107240 \\
$\mathrm{Al}$ & -3.577288 & -0.372727 & 1.993877 \\
\hline & & & \\
\end{tabular}

Table B.4. Atomic coordinates for the $\mathrm{Al}_{33}$ structure, obtained from Ref. [129]. 


\begin{tabular}{lrrr}
\hline $33 \mathrm{Ga}$ & & & \\
xyz & & & \\
$\mathrm{Ga}$ & 0.785818 & 5.269021 & 1.454023 \\
$\mathrm{Ga}$ & 2.212178 & 3.052621 & 0.913968 \\
$\mathrm{Ga}$ & 1.637869 & -0.387070 & -2.829815 \\
$\mathrm{Ga}$ & 4.117361 & -0.003288 & -1.635660 \\
$\mathrm{Ga}$ & 0.467319 & 3.900088 & -1.170155 \\
$\mathrm{Ga}$ & 2.407462 & 2.011946 & -1.588924 \\
$\mathrm{Ga}$ & 0.056038 & 1.934026 & -3.025046 \\
$\mathrm{Ga}$ & 3.811398 & 0.831242 & 0.867369 \\
$\mathrm{Ga}$ & 4.920063 & -1.966687 & 0.171300 \\
$\mathrm{Ga}$ & 3.240460 & -2.590666 & -2.090927 \\
$\mathrm{Ga}$ & 2.409615 & -3.275693 & 0.518987 \\
$\mathrm{Ga}$ & -2.702078 & 0.993453 & -3.491700 \\
$\mathrm{Ga}$ & -2.728154 & -3.719859 & 1.344891 \\
$\mathrm{Ga}$ & -2.251933 & 2.847072 & -1.559024 \\
$\mathrm{Ga}$ & 0.645739 & -2.872923 & -1.952359 \\
$\mathrm{Ga}$ & -1.925274 & -3.155054 & -1.441876 \\
$\mathrm{Ga}$ & -0.146097 & -3.865485 & 0.594250 \\
$\mathrm{Ga}$ & -3.658706 & -1.458832 & -2.715822 \\
$\mathrm{Ga}$ & -0.960139 & -0.604866 & -2.070641 \\
$\mathrm{Ga}$ & -0.228641 & 1.345872 & -0.044048 \\
$\mathrm{Ga}$ & -1.257636 & -1.164861 & 0.546888 \\
$\mathrm{Ga}$ & 1.765684 & -0.756704 & -0.109136 \\
$\mathrm{Ga}$ & -1.628590 & 4.455264 & 0.522912 \\
$\mathrm{Ga}$ & -4.234610 & -2.006742 & -0.029398 \\
$\mathrm{Ga}$ & -3.170189 & 0.456293 & -0.673649 \\
$\mathrm{Ga}$ & 1.473686 & 0.795817 & 2.321106 \\
$\mathrm{Ga}$ & 0.816156 & -1.814819 & 2.447039 \\
$\mathrm{Ga}$ & -0.209987 & 2.906391 & 2.425782 \\
$\mathrm{Ga}$ & 3.511375 & -1.445077 & 2.481325 \\
$\mathrm{Ga}$ & -1.053533 & 0.513583 & 3.189485 \\
$\mathrm{Ga}$ & -2.624028 & 2.057450 & 1.358064 \\
$\mathrm{Ga}$ & -1.824305 & -1.977687 & 3.177536 \\
$\mathrm{Ga}$ & -3.674319 & -0.303825 & 2.093256 \\
\hline & & & \\
& & & \\
$\mathrm{Ga}$ & & \\
$\mathrm{Ga}$ & & & \\
$\mathrm{Ga}$ & & & \\
$\mathrm{Ga}$ & & & \\
$\mathrm{Ga}$ & & & \\
$\mathrm{Ga}$ & & & \\
$\mathrm{Ga}$ & & & \\
$\mathrm{Ga}$ & & & \\
$\mathrm{Ga}$ & & &
\end{tabular}

Table B.5. Atomic coordinates for the $\mathrm{Ga}_{33}$ structure, optimized from the $\mathrm{Al}_{32}$ structure, as listed in Tab. B.2, using the SC PAW for gallium. 


\begin{tabular}{lrrr}
\hline $20 \mathrm{Ga}$ & & & \\
xyz & & & \\
$\mathrm{Ga}$ & -2.450943 & -0.019468 & 2.640399 \\
$\mathrm{Ga}$ & -2.395290 & 2.286768 & 1.333699 \\
$\mathrm{Ga}$ & 0.220089 & 0.794540 & 2.344986 \\
$\mathrm{Ga}$ & -3.600121 & -0.033774 & -0.000002 \\
$\mathrm{Ga}$ & -2.395284 & 2.286773 & -1.333712 \\
$\mathrm{Ga}$ & -2.450942 & -0.019463 & -2.640398 \\
$\mathrm{Ga}$ & -1.145058 & -0.043449 & 0.000002 \\
$\mathrm{Ga}$ & 0.305132 & -2.018452 & -1.482676 \\
$\mathrm{Ga}$ & 0.305135 & -2.018455 & 1.482680 \\
$\mathrm{Ga}$ & -2.452543 & -2.306830 & -1.317772 \\
$\mathrm{Ga}$ & -2.452540 & -2.306825 & 1.317752 \\
$\mathrm{Ga}$ & 3.815364 & -0.000717 & 0.000004 \\
$\mathrm{Ga}$ & 2.547334 & 2.059451 & -1.459429 \\
$\mathrm{Ga}$ & 2.547327 & 2.059448 & 1.459445 \\
$\mathrm{Ga}$ & 2.646906 & -2.538920 & -0.000001 \\
$\mathrm{Ga}$ & 0.220093 & 0.794543 & -2.344982 \\
$\mathrm{Ga}$ & 2.587347 & -0.716819 & -2.444400 \\
$\mathrm{Ga}$ & 0.211414 & 2.478077 & 0.000002 \\
$\mathrm{Ga}$ & 2.587344 & -0.716822 & 2.444400 \\
$\mathrm{Ga}$ & 1.349236 & -0.019607 & 0.000001 \\
\hline & & & \\
\hline
\end{tabular}

Table B.6. Atomic coordinates for the initial SC $\mathrm{Ga}_{20}$ stacked-plane structure. These also represent the coordinates for Fig. 5.3, SC Ga $\mathrm{G}_{20}$ column, ST structure. 


\begin{tabular}{lrrr}
\hline $20 \mathrm{Ga}$ & & & \\
xyz & & & \\
$\mathrm{Ga}$ & -2.991283 & 1.048081 & -0.595450 \\
$\mathrm{Ga}$ & -1.814108 & 2.426348 & 1.617219 \\
$\mathrm{Ga}$ & 1.396662 & 1.391345 & 2.850734 \\
$\mathrm{Ga}$ & 2.885878 & -1.405954 & -0.336849 \\
$\mathrm{Ga}$ & -1.030432 & 2.767188 & -1.076312 \\
$\mathrm{Ga}$ & 1.583875 & 2.281261 & -1.535238 \\
$\mathrm{Ga}$ & -1.757044 & -2.622933 & 0.367289 \\
$\mathrm{Ga}$ & -2.796905 & -0.510141 & 1.505030 \\
$\mathrm{Ga}$ & 2.156181 & -1.064281 & 2.312939 \\
$\mathrm{Ga}$ & 2.945050 & 1.138255 & 0.451406 \\
$\mathrm{Ga}$ & 0.111807 & -1.190936 & -4.353564 \\
$\mathrm{Ga}$ & -0.000372 & 0.051095 & 0.213465 \\
$\mathrm{Ga}$ & -0.760729 & 0.834834 & -2.824313 \\
$\mathrm{Ga}$ & 0.769942 & 3.066215 & 0.966340 \\
$\mathrm{Ga}$ & 0.933118 & -2.956238 & 0.516529 \\
$\mathrm{Ga}$ & 0.181491 & -2.396432 & -1.936582 \\
$\mathrm{Ga}$ & 1.820769 & -0.176017 & -2.326565 \\
$\mathrm{Ga}$ & -0.363936 & -1.993349 & 2.714248 \\
$\mathrm{Ga}$ & -1.029589 & 0.479501 & 3.251635 \\
$\mathrm{Ga}$ & -2.240351 & -1.167867 & -1.781951 \\
\hline & & & \\
\hline
\end{tabular}

Table B.7. Atomic coordinates for the initial SC $\mathrm{Ga}_{20}$ capped-sphere structure. 


\begin{tabular}{lrrr}
\hline $20 \mathrm{Ga}$ & & & \\
xyz & & & \\
$\mathrm{Ga}$ & 1.025482 & -3.098212 & -1.278673 \\
$\mathrm{Ga}$ & 3.851883 & -4.568960 & -0.788043 \\
$\mathrm{Ga}$ & 2.062860 & -6.115168 & -2.045458 \\
$\mathrm{Ga}$ & -0.298719 & -3.717904 & 1.710196 \\
$\mathrm{Ga}$ & 0.295459 & -3.157442 & -4.383698 \\
$\mathrm{Ga}$ & -0.278331 & -5.333826 & -3.001058 \\
$\mathrm{Ga}$ & 0.971790 & -6.004664 & 0.336075 \\
$\mathrm{Ga}$ & 2.193819 & -0.128788 & -1.412653 \\
$\mathrm{Ga}$ & -2.764546 & -0.368956 & -1.631989 \\
$\mathrm{Ga}$ & 3.406061 & -1.863729 & 0.093686 \\
$\mathrm{Ga}$ & -1.311411 & -5.026450 & -0.618742 \\
$\mathrm{Ga}$ & -0.216130 & -0.224469 & -0.350612 \\
$\mathrm{Ga}$ & -0.443376 & -0.747566 & -3.043610 \\
$\mathrm{Ga}$ & 3.881854 & -2.523314 & -2.451187 \\
$\mathrm{Ga}$ & 2.067662 & -1.199267 & -3.856089 \\
$\mathrm{Ga}$ & 1.160515 & -1.463435 & 1.476847 \\
$\mathrm{Ga}$ & -1.756770 & -3.059226 & -2.567404 \\
$\mathrm{Ga}$ & -1.842148 & -2.373860 & 0.017124 \\
$\mathrm{Ga}$ & 2.693000 & -4.383663 & -4.037862 \\
$\mathrm{Ga}$ & 2.438375 & -4.030132 & 1.389920 \\
\hline & & & \\
\hline
\end{tabular}

Table B.8. Atomic coordinates for Fig. 5.3, SC Ga 20 column, PT2 structure. 


\begin{tabular}{lrrr}
\hline $20 \mathrm{Ga}$ & & & \\
xyz & & & \\
$\mathrm{Ga}$ & -3.878831 & -0.150638 & -0.133273 \\
$\mathrm{Ga}$ & -2.125274 & -0.821618 & -2.653514 \\
$\mathrm{Ga}$ & -2.743322 & 0.708660 & 2.071036 \\
$\mathrm{Ga}$ & 1.807195 & 0.015789 & 0.017363 \\
$\mathrm{Ga}$ & 2.722702 & -0.752881 & -2.290763 \\
$\mathrm{Ga}$ & 2.525816 & 0.897690 & 2.560511 \\
$\mathrm{Ga}$ & -0.011796 & 0.407256 & 2.385226 \\
$\mathrm{Ga}$ & 2.517136 & -2.641832 & -0.525144 \\
$\mathrm{Ga}$ & -2.108972 & -1.812847 & 2.172328 \\
$\mathrm{Ga}$ & 0.291847 & -2.141524 & 1.074366 \\
$\mathrm{Ga}$ & 2.855337 & -1.582271 & 1.796341 \\
$\mathrm{Ga}$ & -1.098268 & 0.037312 & 0.004225 \\
$\mathrm{Ga}$ & 0.205480 & -1.783988 & -1.673552 \\
$\mathrm{Ga}$ & -2.265935 & -2.321431 & -0.422054 \\
$\mathrm{Ga}$ & -2.544982 & 1.604975 & -1.647921 \\
$\mathrm{Ga}$ & 2.547169 & 1.842154 & -1.970286 \\
$\mathrm{Ga}$ & 0.374983 & 2.318019 & 0.295464 \\
$\mathrm{Ga}$ & 0.091331 & 1.047740 & -2.199029 \\
$\mathrm{Ga}$ & 3.001465 & 2.325867 & 0.488347 \\
$\mathrm{Ga}$ & -2.163079 & 2.803569 & 0.650330 \\
\hline & & & \\
\hline & & &
\end{tabular}

Table B.9. Atomic coordinates for Fig. 5.3, $\mathrm{SC} \mathrm{Ga}_{20}$ column, GS structure. 


\begin{tabular}{lrrr}
\hline $20 \mathrm{Ga}$ & & & \\
xyz & & & \\
$\mathrm{Ga}$ & -1.980172 & -3.208260 & 0.512872 \\
$\mathrm{Ga}$ & -0.991119 & -2.361773 & 2.778315 \\
$\mathrm{Ga}$ & 1.163330 & -1.265760 & 1.803468 \\
$\mathrm{Ga}$ & -1.373397 & 0.152165 & 3.472547 \\
$\mathrm{Ga}$ & 0.330524 & 1.519483 & 1.833391 \\
$\mathrm{Ga}$ & -2.676941 & 1.832964 & 1.963510 \\
$\mathrm{Ga}$ & -1.830478 & -0.579872 & 0.952147 \\
$\mathrm{Ga}$ & -1.154255 & -0.269968 & -2.098093 \\
$\mathrm{Ga}$ & 0.200563 & -2.306907 & -0.626270 \\
$\mathrm{Ga}$ & -3.751997 & 0.880416 & -0.274894 \\
$\mathrm{Ga}$ & -3.236922 & -1.562782 & -1.116144 \\
$\mathrm{Ga}$ & 3.088930 & -1.403431 & -0.553090 \\
$\mathrm{Ga}$ & 1.121845 & 0.936679 & -3.127041 \\
$\mathrm{Ga}$ & 0.682929 & 3.174029 & -1.739506 \\
$\mathrm{Ga}$ & 3.261577 & 0.373088 & 1.509786 \\
$\mathrm{Ga}$ & -1.226886 & 1.925923 & -0.431258 \\
$\mathrm{Ga}$ & 1.634427 & -1.634567 & -2.826832 \\
$\mathrm{Ga}$ & 3.396760 & 1.001296 & -1.749127 \\
$\mathrm{Ga}$ & 2.377315 & 2.502256 & 0.206602 \\
$\mathrm{Ga}$ & 0.963968 & 0.295021 & -0.490380 \\
\hline & & & \\
\hline
\end{tabular}

Table B.10. Atomic coordinates for Fig. 5.3, $\mathrm{SC} \mathrm{Ga}_{20}$ column, PT structure. 


\begin{tabular}{lrrr}
\hline $20 \mathrm{Ga}$ & & & \\
xyz & & & \\
$\mathrm{Ga}$ & -3.878831 & -0.150638 & -0.133273 \\
$\mathrm{Ga}$ & -2.125274 & -0.821618 & -2.653514 \\
$\mathrm{Ga}$ & -2.743322 & 0.708660 & 2.071036 \\
$\mathrm{Ga}$ & 1.807195 & 0.015789 & 0.017363 \\
$\mathrm{Ga}$ & 2.722702 & -0.752881 & -2.290763 \\
$\mathrm{Ga}$ & 2.525816 & 0.897690 & 2.560511 \\
$\mathrm{Ga}$ & -0.011796 & 0.407256 & 2.385226 \\
$\mathrm{Ga}$ & 2.517136 & -2.641832 & -0.525144 \\
$\mathrm{Ga}$ & -2.108972 & -1.812847 & 2.172328 \\
$\mathrm{Ga}$ & 0.291847 & -2.141524 & 1.074366 \\
$\mathrm{Ga}$ & 2.855337 & -1.582271 & 1.796341 \\
$\mathrm{Ga}$ & -1.098268 & 0.037312 & 0.004225 \\
$\mathrm{Ga}$ & 0.205480 & -1.783988 & -1.673552 \\
$\mathrm{Ga}$ & -2.265935 & -2.321431 & -0.422054 \\
$\mathrm{Ga}$ & -2.544982 & 1.604975 & -1.647921 \\
$\mathrm{Ga}$ & 2.547169 & 1.842154 & -1.970286 \\
$\mathrm{Ga}$ & 0.374983 & 2.318019 & 0.295464 \\
$\mathrm{Ga}$ & 0.091331 & 1.047740 & -2.199029 \\
$\mathrm{Ga}$ & 3.001465 & 2.325867 & 0.488347 \\
$\mathrm{Ga}$ & -2.163079 & 2.803569 & 0.650330 \\
\hline & & & \\
\hline & & &
\end{tabular}

Table B.11. Atomic coordinates for Fig. 5.3, $\mathrm{LC} \mathrm{Ga}_{20}$ column, ST structure. 


\begin{tabular}{lrrr}
\hline $20 \mathrm{Ga}$ & & & \\
xyz & & & \\
$\mathrm{Ga}$ & -2.933729 & 0.991182 & -0.700621 \\
$\mathrm{Ga}$ & -1.896249 & 2.360202 & 1.540369 \\
$\mathrm{Ga}$ & 1.469038 & 1.303277 & 2.812659 \\
$\mathrm{Ga}$ & 2.838097 & -1.357358 & -0.395289 \\
$\mathrm{Ga}$ & -1.002303 & 2.807205 & -1.024764 \\
$\mathrm{Ga}$ & 1.602141 & 2.290896 & -1.437409 \\
$\mathrm{Ga}$ & -1.709306 & -2.609880 & 0.319497 \\
$\mathrm{Ga}$ & -2.746232 & -0.500988 & 1.426119 \\
$\mathrm{Ga}$ & 2.182454 & -1.167452 & 2.329642 \\
$\mathrm{Ga}$ & 2.893370 & 1.097757 & 0.480223 \\
$\mathrm{Ga}$ & -0.283490 & -1.315646 & -4.235252 \\
$\mathrm{Ga}$ & 0.015583 & 0.068761 & 0.226664 \\
$\mathrm{Ga}$ & -0.728050 & 0.971484 & -2.887429 \\
$\mathrm{Ga}$ & 0.736925 & 3.106597 & 1.081141 \\
$\mathrm{Ga}$ & 0.941579 & -2.942055 & 0.623366 \\
$\mathrm{Ga}$ & 0.384096 & -2.449546 & -1.890742 \\
$\mathrm{Ga}$ & 1.740371 & -0.119938 & -2.408832 \\
$\mathrm{Ga}$ & -0.466713 & -1.928170 & 2.737187 \\
$\mathrm{Ga}$ & -0.995639 & 0.574310 & 3.233741 \\
$\mathrm{Ga}$ & -2.041920 & -1.180665 & -1.830260 \\
\hline & & & \\
\hline
\end{tabular}

Table B.12. Atomic coordinates for Fig. 5.3, $\mathrm{LC} \mathrm{Ga}_{20}$ column, CS structure. 


\begin{tabular}{lrrr}
\hline $20 \mathrm{Ga}$ & & & \\
xyz & & & \\
$\mathrm{Ga}$ & -2.413892 & -0.039321 & 2.607831 \\
$\mathrm{Ga}$ & -2.308782 & 2.224810 & 1.314768 \\
$\mathrm{Ga}$ & 0.203823 & 0.812651 & 2.329600 \\
$\mathrm{Ga}$ & -3.629824 & 0.027083 & 0.000000 \\
$\mathrm{Ga}$ & -2.308782 & 2.224810 & -1.314768 \\
$\mathrm{Ga}$ & -2.413892 & -0.039321 & -2.607831 \\
$\mathrm{Ga}$ & -1.152637 & -0.085281 & 0.000000 \\
$\mathrm{Ga}$ & 0.290868 & -2.010168 & -1.495957 \\
$\mathrm{Ga}$ & 0.290868 & -2.010168 & 1.495957 \\
$\mathrm{Ga}$ & -2.435435 & -2.290019 & -1.301258 \\
$\mathrm{Ga}$ & -2.435435 & -2.290019 & 1.301258 \\
$\mathrm{Ga}$ & 3.840901 & 0.012332 & 0.000000 \\
$\mathrm{Ga}$ & 2.516958 & 2.011401 & -1.425309 \\
$\mathrm{Ga}$ & 2.516958 & 2.011401 & 1.425309 \\
$\mathrm{Ga}$ & 2.591310 & -2.442264 & 0.000000 \\
$\mathrm{Ga}$ & 0.203823 & 0.812651 & -2.329600 \\
$\mathrm{Ga}$ & 2.547559 & -0.692905 & -2.358016 \\
$\mathrm{Ga}$ & 0.195608 & 2.462496 & 0.000000 \\
$\mathrm{Ga}$ & 2.547559 & -0.692905 & 2.358016 \\
$\mathrm{Ga}$ & 1.352444 & -0.007265 & 0.000000 \\
\hline & & & \\
\hline
\end{tabular}

Table B.13. Atomic coordinates for Fig. 5.3, $\mathrm{LC} \mathrm{Ga}_{20}$ column, GS structure. 


\begin{tabular}{lrrr}
\hline $20 \mathrm{Ga}$ & & & \\
xyz & & & \\
$\mathrm{Ga}$ & 0.749357 & -3.726895 & -0.654065 \\
$\mathrm{Ga}$ & -0.907258 & -3.365137 & 1.321034 \\
$\mathrm{Ga}$ & -0.750985 & -0.669422 & 2.092292 \\
$\mathrm{Ga}$ & -3.077623 & -1.928942 & 0.854983 \\
$\mathrm{Ga}$ & -2.312048 & 0.805589 & 0.237735 \\
$\mathrm{Ga}$ & -3.429548 & -0.870069 & -1.519392 \\
$\mathrm{Ga}$ & -0.910748 & -1.597410 & -0.768387 \\
$\mathrm{Ga}$ & 1.771772 & -0.348933 & -1.546004 \\
$\mathrm{Ga}$ & 1.569294 & -1.698588 & 0.830133 \\
$\mathrm{Ga}$ & -1.490723 & -0.857099 & -3.268214 \\
$\mathrm{Ga}$ & 0.643950 & -2.349143 & -2.849190 \\
$\mathrm{Ga}$ & 3.320697 & 0.726449 & 0.526327 \\
$\mathrm{Ga}$ & 2.001477 & 0.020167 & 2.833690 \\
$\mathrm{Ga}$ & -0.757023 & 3.346616 & -0.059981 \\
$\mathrm{Ga}$ & -1.342991 & 2.164927 & 2.331750 \\
$\mathrm{Ga}$ & -0.708192 & 1.280843 & -1.910192 \\
$\mathrm{Ga}$ & 1.244516 & 2.527698 & 2.652487 \\
$\mathrm{Ga}$ & 1.799238 & 2.332432 & -2.005083 \\
$\mathrm{Ga}$ & 2.042826 & 3.264446 & 0.434606 \\
$\mathrm{Ga}$ & 0.544010 & 0.942479 & 0.465475 \\
\hline & & & \\
\hline
\end{tabular}

Table B.14. Atomic coordinates for Fig. 5.3, $\mathrm{LC} \mathrm{Ga}_{20}$ column, PT structure. 


\begin{tabular}{lrrr}
\hline $20 \mathrm{Ga}$ & & & \\
xyz & & & \\
$\mathrm{Ga}$ & -3.995037 & -0.144596 & -0.140019 \\
$\mathrm{Ga}$ & -2.180284 & -0.852114 & -2.745489 \\
$\mathrm{Ga}$ & -2.788602 & 0.697371 & 2.133964 \\
$\mathrm{Ga}$ & 1.726455 & 0.019767 & 0.027690 \\
$\mathrm{Ga}$ & 2.732274 & -0.764379 & -2.342053 \\
$\mathrm{Ga}$ & 2.547023 & 0.879143 & 2.610700 \\
$\mathrm{Ga}$ & -0.034526 & 0.471334 & 2.444359 \\
$\mathrm{Ga}$ & 2.653644 & -2.668063 & -0.566432 \\
$\mathrm{Ga}$ & -2.148974 & -1.837487 & 2.229589 \\
$\mathrm{Ga}$ & 0.294880 & -2.136404 & 1.136324 \\
$\mathrm{Ga}$ & 2.922679 & -1.598737 & 1.821497 \\
$\mathrm{Ga}$ & -1.091647 & 0.041113 & -0.002474 \\
$\mathrm{Ga}$ & 0.201224 & -1.820751 & -1.729656 \\
$\mathrm{Ga}$ & -2.281820 & -2.307170 & -0.426540 \\
$\mathrm{Ga}$ & -2.565474 & 1.604214 & -1.685448 \\
$\mathrm{Ga}$ & 2.644368 & 1.824283 & -2.030425 \\
$\mathrm{Ga}$ & 0.376176 & 2.384447 & 0.302002 \\
$\mathrm{Ga}$ & 0.096327 & 1.094398 & -2.223178 \\
$\mathrm{Ga}$ & 3.081727 & 2.333013 & 0.503430 \\
$\mathrm{Ga}$ & -2.190412 & 2.780615 & 0.682159 \\
\hline & & & \\
\hline
\end{tabular}

Table B.15. Atomic coordinates for Fig. 5.3, SC Ga ${ }_{20}^{+}$column, ST structure. 


\begin{tabular}{lrrr}
\hline $20 \mathrm{Ga}$ & & & \\
$\mathrm{xyz}$ & & & \\
$\mathrm{Ga}$ & 1.025482 & -3.098212 & -1.278673 \\
$\mathrm{Ga}$ & 3.851883 & -4.568960 & -0.788043 \\
$\mathrm{Ga}$ & 2.062860 & -6.115168 & -2.045458 \\
$\mathrm{Ga}$ & -0.298719 & -3.717904 & 1.710196 \\
$\mathrm{Ga}$ & 0.295459 & -3.157442 & -4.383698 \\
$\mathrm{Ga}$ & -0.278331 & -5.333826 & -3.001058 \\
$\mathrm{Ga}$ & 0.971790 & -6.004664 & 0.336075 \\
$\mathrm{Ga}$ & 2.193819 & -0.128788 & -1.412653 \\
$\mathrm{Ga}$ & -2.764546 & -0.368956 & -1.631989 \\
$\mathrm{Ga}$ & 3.406061 & -1.863729 & 0.093686 \\
$\mathrm{Ga}$ & -1.311411 & -5.026450 & -0.618742 \\
$\mathrm{Ga}$ & -0.216130 & -0.224469 & -0.350612 \\
$\mathrm{Ga}$ & -0.443376 & -0.747566 & -3.043610 \\
$\mathrm{Ga}$ & 3.881854 & -2.523314 & -2.451187 \\
$\mathrm{Ga}$ & 2.067662 & -1.199267 & -3.856089 \\
$\mathrm{Ga}$ & 1.160515 & -1.463435 & 1.476847 \\
$\mathrm{Ga}$ & -1.756770 & -3.059226 & -2.567404 \\
$\mathrm{Ga}$ & -1.842148 & -2.373860 & 0.017124 \\
$\mathrm{Ga}$ & 2.693000 & -4.383663 & -4.037862 \\
$\mathrm{Ga}$ & 2.438375 & -4.030132 & 1.389920 \\
\hline
\end{tabular}

Table B.16. Atomic coordinates for Fig. 5.3, SC Ga ${ }_{20}^{+}$column, PT2 structure. 


\begin{tabular}{lrrr}
\hline $20 \mathrm{Ga}$ & & & \\
xyz & & & \\
$\mathrm{Ga}$ & -0.845762 & 1.070861 & 1.075903 \\
$\mathrm{Ga}$ & 1.012144 & 2.518604 & 2.496202 \\
$\mathrm{Ga}$ & -0.184134 & 3.703463 & 0.521891 \\
$\mathrm{Ga}$ & -2.585370 & 2.949815 & -0.073394 \\
$\mathrm{Ga}$ & 2.761954 & -1.609961 & -2.227255 \\
$\mathrm{Ga}$ & 0.529800 & -0.673668 & -0.718376 \\
$\mathrm{Ga}$ & -1.990927 & -1.322309 & -0.154315 \\
$\mathrm{Ga}$ & -1.458288 & 1.138641 & -1.726189 \\
$\mathrm{Ga}$ & -1.273479 & -1.284829 & -2.930552 \\
$\mathrm{Ga}$ & 0.685743 & -3.033565 & -2.444266 \\
$\mathrm{Ga}$ & -0.137208 & -3.453730 & 0.048248 \\
$\mathrm{Ga}$ & 1.130976 & 1.930336 & -0.983174 \\
$\mathrm{Ga}$ & 0.985185 & 0.307107 & -3.305574 \\
$\mathrm{Ga}$ & -2.512754 & -0.426755 & 2.805987 \\
$\mathrm{Ga}$ & -0.276221 & 0.620471 & 3.811618 \\
$\mathrm{Ga}$ & 3.463843 & 0.505123 & -0.827758 \\
$\mathrm{Ga}$ & -0.054547 & -1.554510 & 1.981551 \\
$\mathrm{Ga}$ & 1.974861 & 0.238698 & 1.456572 \\
$\mathrm{Ga}$ & 2.358287 & -2.320308 & 0.486408 \\
$\mathrm{Ga}$ & -3.584104 & 0.696517 & 0.706471 \\
\hline & & & \\
\hline & & &
\end{tabular}

Table B.17. Atomic coordinates for Fig. 5.3, $\mathrm{SC} \mathrm{Ga}_{20}^{+}$column, PT structure. 


\begin{tabular}{lrrr}
\hline $20 \mathrm{Ga}$ & & & \\
xyz & & & \\
$\mathrm{Ga}$ & -2.922132 & 1.009584 & -0.765906 \\
$\mathrm{Ga}$ & -1.915011 & 2.283679 & 1.516188 \\
$\mathrm{Ga}$ & 1.452601 & 1.302771 & 2.826620 \\
$\mathrm{Ga}$ & 2.826204 & -1.402735 & -0.469202 \\
$\mathrm{Ga}$ & -0.985093 & 2.920387 & -0.927238 \\
$\mathrm{Ga}$ & 1.604562 & 2.300504 & -1.531126 \\
$\mathrm{Ga}$ & -1.695587 & -2.686097 & 0.281061 \\
$\mathrm{Ga}$ & -2.747503 & -0.536083 & 1.330917 \\
$\mathrm{Ga}$ & 2.206072 & -1.093069 & 2.243732 \\
$\mathrm{Ga}$ & 2.875237 & 1.069820 & 0.406804 \\
$\mathrm{Ga}$ & -0.273387 & -1.200394 & -3.889174 \\
$\mathrm{Ga}$ & 0.010877 & 0.044830 & 0.148487 \\
$\mathrm{Ga}$ & -0.813721 & 1.161544 & -2.864616 \\
$\mathrm{Ga}$ & 0.832488 & 3.060559 & 1.043611 \\
$\mathrm{Ga}$ & 0.974955 & -2.970942 & 0.755549 \\
$\mathrm{Ga}$ & 0.437943 & -2.614708 & -1.787477 \\
$\mathrm{Ga}$ & 1.817156 & -0.102320 & -2.461608 \\
$\mathrm{Ga}$ & -0.530767 & -1.903860 & 2.708349 \\
$\mathrm{Ga}$ & -1.036089 & 0.573002 & 3.243064 \\
$\mathrm{Ga}$ & -2.118784 & -1.216496 & -1.808027 \\
\hline & & & \\
\hline
\end{tabular}

Table B.18. Atomic coordinates for Fig. 5.3, $\mathrm{LC} \mathrm{Ga}_{20}^{+}$column, ST structure. 


\begin{tabular}{lrrr}
\hline $20 \mathrm{Ga}$ & & & \\
xyz & & & \\
$\mathrm{Ga}$ & -0.809484 & 0.592037 & -3.060306 \\
$\mathrm{Ga}$ & 0.019729 & 3.367044 & 0.669921 \\
$\mathrm{Ga}$ & -0.384260 & 1.854001 & 2.719651 \\
$\mathrm{Ga}$ & 2.370985 & -1.597296 & 1.405847 \\
$\mathrm{Ga}$ & -1.224684 & 2.705697 & -1.487685 \\
$\mathrm{Ga}$ & 2.312259 & 1.034444 & 2.173729 \\
$\mathrm{Ga}$ & -1.137392 & -3.876466 & -0.557519 \\
$\mathrm{Ga}$ & -2.889216 & 0.375591 & -1.364525 \\
$\mathrm{Ga}$ & -2.157783 & -0.375925 & 2.363848 \\
$\mathrm{Ga}$ & 0.408799 & -0.661726 & 3.126933 \\
$\mathrm{Ga}$ & 1.566509 & -0.770356 & -2.660393 \\
$\mathrm{Ga}$ & 0.035791 & 0.144162 & 0.020895 \\
$\mathrm{Ga}$ & 1.419507 & 1.950198 & -2.302549 \\
$\mathrm{Ga}$ & 2.418932 & 2.477541 & 0.027358 \\
$\mathrm{Ga}$ & -0.270399 & -2.699849 & 1.628567 \\
$\mathrm{Ga}$ & 1.336803 & -2.602128 & -0.792356 \\
$\mathrm{Ga}$ & 3.068702 & -0.081112 & -0.622491 \\
$\mathrm{Ga}$ & -2.606867 & -1.667648 & 0.180284 \\
$\mathrm{Ga}$ & -2.431710 & 1.738596 & 0.831586 \\
$\mathrm{Ga}$ & -1.046223 & -1.906805 & -2.300797 \\
\hline & & & \\
\hline
\end{tabular}

Table B.19. Atomic coordinates for Fig. 5.3, $\mathrm{LC} \mathrm{Ga}_{20}^{+}$column, PT structure. 


\begin{tabular}{lrrr}
\hline $20 \mathrm{Ga}$ & & & \\
xyz & & & \\
$\mathrm{Ga}$ & 1.138850 & -1.019324 & -1.953106 \\
$\mathrm{Ga}$ & -0.731407 & 3.349739 & -0.837134 \\
$\mathrm{Ga}$ & -0.053832 & -3.459855 & -1.671554 \\
$\mathrm{Ga}$ & 0.020506 & -3.569441 & 0.936542 \\
$\mathrm{Ga}$ & -0.807940 & 0.922664 & -2.139886 \\
$\mathrm{Ga}$ & 1.899899 & 0.509379 & 2.903419 \\
$\mathrm{Ga}$ & -3.243708 & -0.305794 & -1.583711 \\
$\mathrm{Ga}$ & 0.702386 & 0.843762 & 0.175491 \\
$\mathrm{Ga}$ & -1.578037 & -1.773459 & -3.004614 \\
$\mathrm{Ga}$ & -3.572753 & -0.759567 & 0.963283 \\
$\mathrm{Ga}$ & 1.674946 & -1.497630 & 0.862564 \\
$\mathrm{Ga}$ & -1.098421 & -1.330919 & -0.233529 \\
$\mathrm{Ga}$ & 1.688026 & 3.535409 & 0.194349 \\
$\mathrm{Ga}$ & -1.936246 & 1.388875 & 0.612491 \\
$\mathrm{Ga}$ & 3.400360 & -0.038915 & -0.873246 \\
$\mathrm{Ga}$ & 0.089269 & 2.495946 & 2.266406 \\
$\mathrm{Ga}$ & 1.809857 & 1.846066 & -2.074520 \\
$\mathrm{Ga}$ & -2.086665 & -2.570534 & 2.044129 \\
$\mathrm{Ga}$ & 3.293810 & 1.788488 & 1.042255 \\
$\mathrm{Ga}$ & -0.608859 & -0.354910 & 2.370393 \\
\hline & & & \\
\hline
\end{tabular}

Table B.20. Atomic coordinates for Fig. 5.3, $\mathrm{LC} \mathrm{Ga}_{20}^{+}$column, PT2 structure. 


\begin{tabular}{lrrr}
\hline $7 \mathrm{Ga}$ & & & \\
xyz & & & \\
$\mathrm{Ga}$ & -1.289912 & -1.300196 & -0.835131 \\
$\mathrm{Ga}$ & -1.553153 & -0.103886 & 1.398669 \\
$\mathrm{Ga}$ & -0.781926 & 1.855803 & -0.037288 \\
$\mathrm{Ga}$ & 1.589559 & 1.063843 & 0.150991 \\
$\mathrm{Ga}$ & 1.190809 & -1.422263 & -0.488524 \\
$\mathrm{Ga}$ & -0.045396 & 0.578934 & -2.253959 \\
$\mathrm{Ga}$ & 0.890019 & -0.672234 & 2.065242 \\
\hline
\end{tabular}

Table B.21. Atomic coordinates for the initial $\mathrm{Ga}_{7}$ structure.

\begin{tabular}{lrrr}
\hline $8 \mathrm{Ga}$ & & & \\
xyz & & & \\
$\mathrm{Ga}$ & -1.364549 & -2.069674 & -1.432328 \\
$\mathrm{Ga}$ & -0.974354 & -0.794874 & 0.930699 \\
$\mathrm{Ga}$ & -0.251478 & 1.272866 & -0.262840 \\
$\mathrm{Ga}$ & 2.204725 & 1.118755 & 0.924348 \\
$\mathrm{Ga}$ & 1.085983 & -2.195557 & -0.272806 \\
$\mathrm{Ga}$ & -0.676823 & 0.020159 & -2.647391 \\
$\mathrm{Ga}$ & 1.782247 & -0.123907 & -1.476135 \\
$\mathrm{Ga}$ & 1.463687 & -0.967528 & 2.115293 \\
\hline
\end{tabular}

Table B.22. Atomic coordinates for the initial $\mathrm{Ga}_{8}$ structure. 


\begin{tabular}{lrrr}
\hline $9 \mathrm{Ga}$ & & & \\
xyz & & & \\
$\mathrm{Ga}$ & -1.222953 & -2.063369 & -1.509341 \\
$\mathrm{Ga}$ & -0.939092 & -1.912361 & 1.237398 \\
$\mathrm{Ga}$ & 0.653276 & 2.436269 & -1.289356 \\
$\mathrm{Ga}$ & 1.752329 & 1.464147 & 1.080626 \\
$\mathrm{Ga}$ & 1.028914 & -2.703034 & -0.225699 \\
$\mathrm{Ga}$ & -0.919761 & 0.350824 & -2.280025 \\
$\mathrm{Ga}$ & 1.373124 & -0.336475 & -0.999783 \\
$\mathrm{Ga}$ & 1.543602 & -0.975761 & 1.865020 \\
$\mathrm{Ga}$ & -0.892592 & 0.451025 & 0.501883 \\
\hline
\end{tabular}

Table B.23. Atomic coordinates for the initial $\mathrm{Ga}_{9}$ structure.

\begin{tabular}{lrrr}
\hline $10 \mathrm{Ga}$ & & & \\
xyz & & & \\
$\mathrm{Ga}$ & -1.291224 & -1.988776 & -1.620851 \\
$\mathrm{Ga}$ & -0.669159 & -2.107362 & 1.534940 \\
$\mathrm{Ga}$ & 1.339019 & 2.320284 & -1.415292 \\
$\mathrm{Ga}$ & 1.387276 & 1.608287 & 1.144625 \\
$\mathrm{Ga}$ & 0.968404 & -2.725843 & -0.448636 \\
$\mathrm{Ga}$ & -1.176305 & 0.454333 & -2.317442 \\
$\mathrm{Ga}$ & 1.203258 & -0.269967 & -1.012039 \\
$\mathrm{Ga}$ & -1.156613 & 2.527154 & -0.660373 \\
$\mathrm{Ga}$ & 1.587121 & -0.782593 & 1.984488 \\
$\mathrm{Ga}$ & -0.971544 & 0.202904 & 0.530929 \\
\hline
\end{tabular}

Table B.24. Atomic coordinates for the initial $\mathrm{Ga}_{10}$ structure. 


\begin{tabular}{lrrr}
\hline $11 \mathrm{Ga}$ & & & \\
xyz & & & \\
$\mathrm{Ga}$ & -1.220233 & 0.761577 & 2.279651 \\
$\mathrm{Ga}$ & -1.129460 & -1.995308 & -1.461286 \\
$\mathrm{Ga}$ & -1.129460 & -1.995308 & 1.461286 \\
$\mathrm{Ga}$ & 1.188461 & 1.945854 & -1.382006 \\
$\mathrm{Ga}$ & 1.188461 & 1.945854 & 1.382006 \\
$\mathrm{Ga}$ & 1.215578 & -2.407571 & -0.000000 \\
$\mathrm{Ga}$ & -1.220233 & 0.761577 & -2.279651 \\
$\mathrm{Ga}$ & 1.198613 & -0.684774 & -2.306161 \\
$\mathrm{Ga}$ & -1.244397 & 2.381125 & 0.000000 \\
$\mathrm{Ga}$ & 1.198613 & -0.684774 & 2.306162 \\
$\mathrm{Ga}$ & -0.045943 & -0.028255 & -0.000000 \\
\hline
\end{tabular}

Table B.25. Atomic coordinates for the initial $\mathrm{Ga}_{11}$ structure.

\begin{tabular}{lrrr}
\hline $\begin{array}{l}12 \mathrm{Ga} \\
\text { xyz }\end{array}$ & & & \\
$\mathrm{Ga}$ & 0.000968 & -0.735658 & -2.448964 \\
$\mathrm{Ga}$ & -2.012798 & -1.522479 & -0.814074 \\
$\mathrm{Ga}$ & -1.732366 & 1.112479 & -1.641393 \\
$\mathrm{Ga}$ & 0.946136 & 1.733948 & -1.739984 \\
$\mathrm{Ga}$ & 2.293939 & -0.475975 & -0.933625 \\
$\mathrm{Ga}$ & 0.482849 & -2.482840 & -0.374164 \\
$\mathrm{Ga}$ & -0.000472 & 0.735892 & 2.449197 \\
$\mathrm{Ga}$ & 2.012866 & 1.522774 & 0.813977 \\
$\mathrm{Ga}$ & 1.732373 & -1.112540 & 1.640889 \\
$\mathrm{Ga}$ & -0.945902 & -1.733528 & 1.740266 \\
$\mathrm{Ga}$ & -2.294086 & 0.475728 & 0.933702 \\
$\mathrm{Ga}$ & -0.483507 & 2.482200 & 0.374172 \\
\hline
\end{tabular}

Table B.26. Atomic coordinates for the initial $\mathrm{Ga}_{12}$ structure. 


\begin{tabular}{lrrr}
\hline $7 \mathrm{Ga}$ & & & \\
xyz & & & \\
$\mathrm{Ga}$ & -0.842366 & -1.716791 & 0.156455 \\
$\mathrm{Ga}$ & 0.491966 & -0.629541 & 2.206831 \\
$\mathrm{Ga}$ & -0.252761 & -0.205870 & -2.304808 \\
$\mathrm{Ga}$ & 1.629360 & -0.992893 & -0.189841 \\
$\mathrm{Ga}$ & -1.922217 & 0.488798 & -0.349773 \\
$\mathrm{Ga}$ & 1.210147 & 1.410033 & -0.773160 \\
$\mathrm{Ga}$ & -0.314129 & 1.646262 & 1.254292 \\
\hline
\end{tabular}

Table B.27. Atomic coordinates for the PT-derived lowest energy structure for $\mathrm{Ga}_{7}$.

\begin{tabular}{lrrr}
\hline $9 \mathrm{Ga}$ & & & \\
xyz & & & \\
$\mathrm{Ga}$ & -1.370053 & 1.748598 & 2.088432 \\
$\mathrm{Ga}$ & -1.279505 & 0.030909 & -1.269277 \\
$\mathrm{Ga}$ & 1.388466 & -1.521898 & 0.698137 \\
$\mathrm{Ga}$ & 1.109861 & 0.920845 & 1.330485 \\
$\mathrm{Ga}$ & -0.804437 & 2.743548 & -0.206636 \\
$\mathrm{Ga}$ & -1.184136 & -0.751526 & 1.084287 \\
$\mathrm{Ga}$ & 1.262086 & -1.211200 & -1.884003 \\
$\mathrm{Ga}$ & 1.142673 & 1.271833 & -1.209313 \\
$\mathrm{Ga}$ & -0.264952 & -3.231105 & -0.632111 \\
\hline
\end{tabular}

Table B.28. Atomic coordinates for the PT-derived lowest energy structure for $\mathrm{Ga}_{9}$. 


\begin{tabular}{lrrr}
\hline $11 \mathrm{Ga}$ & & & \\
xyz & & & \\
$\mathrm{Ga}$ & -1.684818 & 1.196975 & 1.820930 \\
$\mathrm{Ga}$ & -0.262046 & -0.853304 & -0.483260 \\
$\mathrm{Ga}$ & 2.415790 & -1.375979 & -0.210067 \\
$\mathrm{Ga}$ & 0.918878 & 0.465074 & 1.675441 \\
$\mathrm{Ga}$ & 2.424532 & 1.225473 & -0.445666 \\
$\mathrm{Ga}$ & -1.455372 & -1.405162 & 1.921456 \\
$\mathrm{Ga}$ & 0.905660 & -2.483434 & 1.612136 \\
$\mathrm{Ga}$ & 1.129343 & 0.329929 & -2.530736 \\
$\mathrm{Ga}$ & -0.186523 & 1.930417 & -0.315767 \\
$\mathrm{Ga}$ & -2.744556 & 0.312074 & -0.396832 \\
$\mathrm{Ga}$ & -1.460888 & 0.657936 & -2.647637 \\
\hline
\end{tabular}

Table B.29. Atomic coordinates for the PT-derived lowest energy structure for $\mathrm{Ga}_{11}$.

\begin{tabular}{lrrr}
\hline $\begin{array}{l}12 \mathrm{Ga} \\
\text { xyz }\end{array}$ & & & \\
$\mathrm{Ga}$ & 1.366667 & 0.356558 & 2.759997 \\
$\mathrm{Ga}$ & 0.846222 & 2.692143 & 1.491101 \\
$\mathrm{Ga}$ & -2.159087 & -1.464214 & 0.873775 \\
$\mathrm{Ga}$ & -0.124846 & 0.879617 & -0.326968 \\
$\mathrm{Ga}$ & -2.998101 & 0.522117 & -0.607382 \\
$\mathrm{Ga}$ & -0.692481 & -1.982235 & -1.236756 \\
$\mathrm{Ga}$ & -1.261080 & 0.255233 & -2.614536 \\
$\mathrm{Ga}$ & -1.250353 & 0.831098 & 2.036638 \\
$\mathrm{Ga}$ & 0.547362 & -1.453866 & 1.249744 \\
$\mathrm{Ga}$ & 1.902847 & -1.645127 & -1.116869 \\
$\mathrm{Ga}$ & 2.423448 & 0.667179 & 0.235334 \\
$\mathrm{Ga}$ & 1.399403 & 0.341501 & -2.744077 \\
\hline
\end{tabular}

Table B.30. Atomic coordinates for the PT-derived lowest energy structure for $\mathrm{Ga}_{12}$. 


\begin{tabular}{|c|c|c|c|}
\hline \multicolumn{4}{|c|}{$32 \mathrm{Ga}$} \\
\hline$x y z$ & & & \\
\hline $\mathrm{Ga}$ & -0.116944 & -3.604583 & 2.033354 \\
\hline $\mathrm{Ga}$ & -0.247000 & -0.327641 & 4.300804 \\
\hline $\mathrm{Ga}$ & -1.848520 & -2.010756 & -4.641936 \\
\hline $\mathrm{Ga}$ & -2.230637 & 0.366519 & -3.015791 \\
\hline $\mathrm{Ga}$ & 2.229565 & 2.753885 & -1.240687 \\
\hline $\mathrm{Ga}$ & -0.361403 & 4.272389 & 1.775902 \\
\hline $\mathrm{Ga}$ & 0.186831 & -3.138138 & -3.113433 \\
\hline $\mathrm{Ga}$ & 2.322153 & -1.928758 & -4.413799 \\
\hline $\mathrm{Ga}$ & -0.232149 & 4.213147 & -1.241240 \\
\hline $\mathrm{Ga}$ & -2.549313 & 0.502659 & 2.530416 \\
\hline $\mathrm{Ga}$ & 2.288668 & -2.351438 & 0.961999 \\
\hline $\mathrm{Ga}$ & -2.443394 & -2.533393 & 0.700076 \\
\hline $\mathrm{Ga}$ & 2.173860 & 0.455086 & 2.840595 \\
\hline $\mathrm{Ga}$ & 0.197961 & -0.147598 & -4.264143 \\
\hline $\mathrm{Ga}$ & 0.092686 & 2.053056 & -2.828485 \\
\hline $\mathrm{Ga}$ & 2.230938 & 2.754755 & 1.594109 \\
\hline $\mathrm{Ga}$ & -2.360400 & -2.205719 & -1.920842 \\
\hline $\mathrm{Ga}$ & 2.527173 & -2.103084 & -1.647118 \\
\hline $\mathrm{Ga}$ & -2.649163 & 2.834994 & 1.203653 \\
\hline $\mathrm{Ga}$ & 0.004206 & -3.488739 & -0.531892 \\
\hline $\mathrm{Ga}$ & -0.280748 & 2.004586 & 3.163668 \\
\hline $\mathrm{Ga}$ & 2.456963 & 0.466955 & -2.737069 \\
\hline $\mathrm{Ga}$ & -2.346065 & 2.580536 & -1.484248 \\
\hline $\mathrm{Ga}$ & -2.184690 & 0.097119 & -0.153541 \\
\hline $\mathrm{Ga}$ & 2.087406 & 0.261094 & 0.065518 \\
\hline $\mathrm{Ga}$ & -0.116087 & 1.830928 & 0.262796 \\
\hline $\mathrm{Ga}$ & 0.047611 & -0.747302 & -1.551257 \\
\hline $\mathrm{Ga}$ & -0.116568 & -0.843344 & 1.521324 \\
\hline $\mathrm{Ga}$ & 1.915763 & -2.074961 & 3.562714 \\
\hline $\mathrm{Ga}$ & -2.287180 & -2.005868 & 3.269588 \\
\hline $\mathrm{Ga}$ & -0.281694 & -3.114127 & 4.689456 \\
\hline $\mathrm{Ga}$ & 1.890171 & 5.177741 & 0.309510 \\
\hline
\end{tabular}

Table B.31. Atomic coordinates for the $\mathrm{Ga}_{32}^{+}$initial structure. 


\begin{tabular}{lrrr}
\hline $33 \mathrm{Ga}$ & & & \\
xyz & & & \\
$\mathrm{Ga}$ & 0.586330 & 5.398514 & 1.335100 \\
$\mathrm{Ga}$ & 2.245239 & 3.276817 & 0.983442 \\
$\mathrm{Ga}$ & 1.457957 & -0.109114 & -2.508465 \\
$\mathrm{Ga}$ & 3.985849 & 0.092918 & -1.603311 \\
$\mathrm{Ga}$ & 0.292500 & 3.936451 & -1.078838 \\
$\mathrm{Ga}$ & 2.425919 & 2.314149 & -1.669701 \\
$\mathrm{Ga}$ & -0.081534 & 2.070094 & -3.118761 \\
$\mathrm{Ga}$ & 3.713178 & 0.987803 & 0.867408 \\
$\mathrm{Ga}$ & 5.054023 & -1.714097 & 0.307411 \\
$\mathrm{Ga}$ & 3.256877 & -2.570205 & -1.832498 \\
$\mathrm{Ga}$ & 2.777432 & -3.444039 & 0.783721 \\
$\mathrm{Ga}$ & -2.816532 & 1.019773 & -3.612885 \\
$\mathrm{Ga}$ & -2.361353 & 2.611527 & -1.414033 \\
$\mathrm{Ga}$ & 0.736755 & -2.760045 & -2.385067 \\
$\mathrm{Ga}$ & -1.805598 & -3.215860 & -1.549488 \\
$\mathrm{Ga}$ & 0.214805 & -3.580280 & 0.305844 \\
$\mathrm{Ga}$ & -3.645596 & -1.644851 & -2.799081 \\
$\mathrm{Ga}$ & -1.118629 & -0.598304 & -2.212934 \\
$\mathrm{Ga}$ & -0.092702 & 1.273876 & -0.263403 \\
$\mathrm{Ga}$ & -1.208795 & -1.047547 & 0.535313 \\
$\mathrm{Ga}$ & 1.808829 & -0.951840 & 0.063930 \\
$\mathrm{Ga}$ & -1.879954 & 4.327885 & 0.605745 \\
$\mathrm{Ga}$ & -3.376578 & 0.250709 & -0.747466 \\
$\mathrm{Ga}$ & 1.331684 & 0.775582 & 2.147303 \\
$\mathrm{Ga}$ & 0.839750 & -1.841335 & 2.598678 \\
$\mathrm{Ga}$ & -0.169481 & 2.881037 & 2.274775 \\
$\mathrm{Ga}$ & 3.462496 & -1.304130 & 2.598060 \\
$\mathrm{Ga}$ & -1.010608 & 0.555414 & 3.637272 \\
$\mathrm{Ga}$ & -2.431795 & 1.585539 & 1.392224 \\
$\mathrm{Ga}$ & -3.937882 & -0.498388 & 2.087700 \\
$\mathrm{Ga}$ & -4.105345 & -2.287812 & -0.006636 \\
$\mathrm{Ga}$ & -1.835340 & -1.920480 & 3.021513 \\
$\mathrm{Ga}$ & -2.311901 & -3.869737 & 1.257151 \\
\hline & & & \\
& & & \\
$\mathrm{Ga}$ & & & \\
$\mathrm{Ga}$ & & & \\
$\mathrm{Ga}$ & & & \\
$\mathrm{Ga}$ & & & \\
$\mathrm{Ga}$ & & & \\
$\mathrm{Ga}$ & & & \\
$\mathrm{Ga}$ & & & \\
$\mathrm{Ga}$ & & & \\
$\mathrm{Ga}$ & & &
\end{tabular}

Table B.32. Atomic coordinates for the $\mathrm{Ga}_{33}^{+}$initial structure. 


\begin{tabular}{lrrr}
\hline $34 \mathrm{Ga}$ & & & \\
xyz & & & \\
$\mathrm{Ga}$ & 0.948644 & 2.429639 & -3.517865 \\
$\mathrm{Ga}$ & 3.329583 & -1.175991 & 2.799841 \\
$\mathrm{Ga}$ & -1.489962 & -0.043484 & 0.766064 \\
$\mathrm{Ga}$ & -4.300358 & 0.047659 & 0.431339 \\
$\mathrm{Ga}$ & 1.996293 & 1.327575 & 3.188788 \\
$\mathrm{Ga}$ & -3.169087 & -2.339569 & 0.837327 \\
$\mathrm{Ga}$ & -1.920875 & 2.864111 & -3.239963 \\
$\mathrm{Ga}$ & 4.300357 & -0.047658 & 0.431341 \\
$\mathrm{Ga}$ & -0.568865 & -3.011892 & 1.389616 \\
$\mathrm{Ga}$ & -1.772820 & -3.359025 & -1.180241 \\
$\mathrm{Ga}$ & 2.154036 & -2.924827 & 1.183510 \\
$\mathrm{Ga}$ & -2.925398 & -0.924927 & -1.823460 \\
$\mathrm{Ga}$ & -0.215455 & 1.313488 & -1.237832 \\
$\mathrm{Ga}$ & -2.154038 & 2.924829 & 1.183508 \\
$\mathrm{Ga}$ & -1.360341 & 0.255597 & -3.699539 \\
$\mathrm{Ga}$ & 0.215456 & -1.313487 & -1.237832 \\
$\mathrm{Ga}$ & 2.925398 & 0.924924 & -1.823461 \\
$\mathrm{Ga}$ & 1.489962 & 0.043482 & 0.766067 \\
$\mathrm{Ga}$ & -0.948644 & -2.429638 & -3.517865 \\
$\mathrm{Ga}$ & -3.139165 & 1.781499 & -1.133504 \\
$\mathrm{Ga}$ & 0.624878 & -1.242130 & 3.189637 \\
$\mathrm{Ga}$ & -3.329582 & 1.175988 & 2.799840 \\
$\mathrm{Ga}$ & 0.887621 & -3.999403 & -0.944015 \\
$\mathrm{Ga}$ & -0.887618 & 3.999404 & -0.944014 \\
$\mathrm{Ga}$ & -1.996295 & -1.327575 & 3.188786 \\
$\mathrm{Ga}$ & -0.624874 & 1.242129 & 3.189637 \\
$\mathrm{Ga}$ & 0.568862 & 3.011889 & 1.389617 \\
$\mathrm{Ga}$ & 4.762029 & 1.171608 & 2.990991 \\
$\mathrm{Ga}$ & 3.169083 & 2.339569 & 0.837327 \\
$\mathrm{Ga}$ & 1.772822 & 3.359025 & -1.180241 \\
$\mathrm{Ga}$ & -4.762029 & -1.171608 & 2.990988 \\
$\mathrm{Ga}$ & 3.139168 & -1.781500 & -1.133496 \\
$\mathrm{Ga}$ & 1.360336 & -0.255595 & -3.699542 \\
$\mathrm{Ga}$ & 1.920877 & -2.864107 & -3.239960 \\
\hline & & & \\
& & &
\end{tabular}

Table B.33. Atomic coordinates for the $\mathrm{Ga}_{34}^{+}$initial structure. 


\begin{tabular}{lrrr}
\hline 35 Ga & & & \\
xyz & & & \\
$\mathrm{Ga}$ & -4.429164 & -0.135755 & -0.283820 \\
$\mathrm{Ga}$ & -2.718211 & -3.539923 & -0.299749 \\
$\mathrm{Ga}$ & 0.082470 & 4.933294 & 1.141599 \\
$\mathrm{Ga}$ & 1.444860 & 2.636293 & 1.968867 \\
$\mathrm{Ga}$ & 2.885989 & -0.035704 & -2.718198 \\
$\mathrm{Ga}$ & 2.542504 & -3.660523 & -0.303452 \\
$\mathrm{Ga}$ & -1.680684 & 4.245093 & -0.828544 \\
$\mathrm{Ga}$ & 0.131266 & 4.784234 & -2.858423 \\
$\mathrm{Ga}$ & 4.424132 & -0.316714 & -0.244413 \\
$\mathrm{Ga}$ & -1.362423 & -2.358628 & 1.909019 \\
$\mathrm{Ga}$ & -2.858890 & 0.095759 & -2.736665 \\
$\mathrm{Ga}$ & -2.787676 & 0.475456 & 1.982916 \\
$\mathrm{Ga}$ & -1.388356 & -2.316495 & -2.526337 \\
$\mathrm{Ga}$ & 1.859904 & 4.169665 & -0.780423 \\
$\mathrm{Ga}$ & 3.140804 & 1.929521 & -0.334007 \\
$\mathrm{Ga}$ & 1.294529 & -2.372416 & -2.516141 \\
$\mathrm{Ga}$ & -1.362616 & 2.700267 & 1.953711 \\
$\mathrm{Ga}$ & -1.387432 & 2.287233 & -2.845109 \\
$\mathrm{Ga}$ & 1.234749 & -2.422971 & 1.900047 \\
$\mathrm{Ga}$ & -3.051633 & 2.063385 & -0.387357 \\
$\mathrm{Ga}$ & -0.091028 & -3.661656 & -0.214382 \\
$\mathrm{Ga}$ & 1.534802 & 2.228107 & -2.809217 \\
$\mathrm{Ga}$ & 2.776270 & 0.348279 & 2.025558 \\
$\mathrm{Ga}$ & -0.005471 & 0.191753 & 1.425839 \\
$\mathrm{Ga}$ & 0.012063 & 0.034235 & -2.105086 \\
$\mathrm{Ga}$ & 1.748253 & -0.888132 & -0.265711 \\
$\mathrm{Ga}$ & 0.039342 & 2.104285 & -0.413062 \\
$\mathrm{Ga}$ & -1.776214 & -0.818749 & -0.277579 \\
$\mathrm{Ga}$ & -4.326211 & -2.251344 & -2.085756 \\
$\mathrm{Ga}$ & -2.885682 & -1.306539 & 4.128360 \\
\hline $\mathrm{Ga}$ & -4.097085 & -2.084699 & 1.690782 \\
$\mathrm{Ga}$ & 3.247564 & -2.433635 & -2.048929 \\
$\mathrm{Ga}$ & -0.003217 & -0.867771 & 3.945536 \\
$\mathrm{Ga}$ & 2.836948 & -1.489354 & 4.126606 \\
$\mathrm{Ga}$ & & \\
$\mathrm{Ga}$ & & & \\
$\mathrm{Ga}$ & & & \\
$\mathrm{Ga}$ & & & \\
$\mathrm{Ga}$ & & & \\
$\mathrm{Ga}$ & & & \\
$\mathrm{Ga}$ & & & \\
$\mathrm{Ga}$ & & & \\
$\mathrm{Ga}$ & & & \\
$\mathrm{Ga}$ & & &
\end{tabular}

Table B.34. Atomic coordinates for the $\mathrm{Ga}_{35}^{+}$initial structure. 


\begin{tabular}{lrrr}
\hline $33 \mathrm{Ga}$ & & & \\
xyz & & & \\
$\mathrm{Ga}$ & 0.564097 & -2.755053 & -2.162355 \\
$\mathrm{Ga}$ & 2.909634 & -2.172788 & -1.046598 \\
$\mathrm{Ga}$ & -2.652642 & 2.443697 & 1.158312 \\
$\mathrm{Ga}$ & 2.050724 & 2.594422 & -1.726275 \\
$\mathrm{Ga}$ & -0.382126 & 2.153851 & -3.081470 \\
$\mathrm{Ga}$ & 0.788391 & 4.223533 & 0.155786 \\
$\mathrm{Ga}$ & -1.277036 & -0.340369 & -3.293527 \\
$\mathrm{Ga}$ & -3.456524 & -0.057412 & 0.802538 \\
$\mathrm{Ga}$ & -1.193181 & -1.170145 & -0.556017 \\
$\mathrm{Ga}$ & 5.177080 & -1.544741 & 0.485370 \\
$\mathrm{Ga}$ & 3.040672 & -2.803438 & 1.787084 \\
$\mathrm{Ga}$ & -1.994799 & 3.813487 & -1.322288 \\
$\mathrm{Ga}$ & -1.589431 & -2.509973 & 1.944792 \\
$\mathrm{Ga}$ & -3.822799 & -3.736977 & -1.762720 \\
$\mathrm{Ga}$ & -2.984601 & 1.445120 & -1.691622 \\
$\mathrm{Ga}$ & -3.621298 & -1.042628 & -1.850988 \\
$\mathrm{Ga}$ & -1.632108 & 5.047562 & 1.167345 \\
$\mathrm{Ga}$ & 1.429568 & -0.636171 & 0.799234 \\
$\mathrm{Ga}$ & -1.848937 & -2.973116 & -3.354068 \\
$\mathrm{Ga}$ & 0.348211 & 4.798254 & -2.504938 \\
$\mathrm{Ga}$ & 0.832589 & -3.570181 & 0.656483 \\
$\mathrm{Ga}$ & -0.351081 & 1.694582 & -0.294824 \\
$\mathrm{Ga}$ & -0.163479 & 2.856294 & 2.393003 \\
$\mathrm{Ga}$ & -1.427948 & -3.915548 & -0.514450 \\
$\mathrm{Ga}$ & -0.931814 & 0.191611 & 1.927724 \\
$\mathrm{Ga}$ & 1.085052 & -0.046843 & -1.853917 \\
$\mathrm{Ga}$ & -3.942523 & -2.698036 & 0.666595 \\
$\mathrm{Ga}$ & 0.668813 & -1.905157 & 3.200520 \\
$\mathrm{Ga}$ & 3.793038 & 0.288746 & 2.007048 \\
$\mathrm{Ga}$ & 2.335513 & 2.302072 & 1.108153 \\
$\mathrm{Ga}$ & 1.405454 & 0.803453 & 3.362727 \\
$\mathrm{Ga}$ & 3.610600 & 0.542059 & -0.768205 \\
$\mathrm{Ga}$ & 3.232891 & -1.320170 & 4.161533 \\
\hline & & &
\end{tabular}

Table B.35. Atomic coordinates for the $\mathrm{Ga}_{33}^{+}$PT-derived structure. 


\begin{tabular}{lrrr}
\hline 34 Ga & & & \\
xyz & & & \\
$\mathrm{Ga}$ & -2.956132 & -2.389504 & 1.676966 \\
$\mathrm{Ga}$ & 3.818990 & -2.101005 & 1.410634 \\
$\mathrm{Ga}$ & 0.404307 & 1.513052 & -0.447321 \\
$\mathrm{Ga}$ & 1.555176 & 1.698308 & -3.509240 \\
$\mathrm{Ga}$ & 3.548766 & -1.643453 & -1.252716 \\
$\mathrm{Ga}$ & -1.138155 & 2.543819 & -3.169563 \\
$\mathrm{Ga}$ & -5.402464 & -1.662796 & 0.489959 \\
$\mathrm{Ga}$ & 1.531964 & -3.653851 & 1.242096 \\
$\mathrm{Ga}$ & -0.229177 & 3.621096 & 1.582859 \\
$\mathrm{Ga}$ & -0.336143 & 4.202181 & -0.984041 \\
$\mathrm{Ga}$ & 3.414385 & 0.566717 & 1.440804 \\
$\mathrm{Ga}$ & -2.554478 & 2.710607 & -0.705494 \\
$\mathrm{Ga}$ & -1.426997 & -0.245273 & 0.665705 \\
$\mathrm{Ga}$ & -0.527233 & -0.098116 & -4.402083 \\
$\mathrm{Ga}$ & -3.967747 & 0.579064 & -0.398976 \\
$\mathrm{Ga}$ & -0.416998 & -2.393217 & 2.700453 \\
$\mathrm{Ga}$ & 1.952073 & -1.244629 & 3.153374 \\
$\mathrm{Ga}$ & 1.209606 & -0.992631 & 0.374199 \\
$\mathrm{Ga}$ & -2.351306 & 2.164041 & 2.018759 \\
$\mathrm{Ga}$ & -1.687384 & 0.246699 & -1.974588 \\
$\mathrm{Ga}$ & 3.396354 & 0.922862 & -1.324065 \\
$\mathrm{Ga}$ & 2.374640 & 3.365541 & -1.396605 \\
$\mathrm{Ga}$ & -1.645106 & -0.171222 & 3.501606 \\
$\mathrm{Ga}$ & -3.137999 & -2.050371 & -1.167226 \\
$\mathrm{Ga}$ & 2.399977 & 2.982410 & 1.236056 \\
$\mathrm{Ga}$ & 1.152132 & -0.600480 & -2.290186 \\
$\mathrm{Ga}$ & -0.961543 & -2.323218 & -2.800983 \\
$\mathrm{Ga}$ & 3.697360 & -4.314223 & -0.407590 \\
$\mathrm{Ga}$ & 1.291841 & -3.333718 & -1.587952 \\
$\mathrm{Ga}$ & -0.915172 & -3.218564 & 0.081749 \\
$\mathrm{Ga}$ & 0.865019 & 4.356194 & -3.590808 \\
$\mathrm{Ga}$ & 0.631226 & 1.149239 & 2.524520 \\
$\mathrm{Ga}$ & -4.044426 & 0.073297 & 2.277329 \\
$\mathrm{Ga}$ & 0.454634 & -0.258853 & 5.032356 \\
\hline & & & \\
\hline
\end{tabular}

Table B.36. Atomic coordinates for the $\mathrm{Ga}_{34}^{+}$PT-derived structure. 


\begin{tabular}{lrrr}
\hline 34 Ga & & & \\
xyz & & & \\
$\mathrm{Ga}$ & 1.948430 & 3.505813 & -0.296626 \\
$\mathrm{Ga}$ & -1.883471 & 3.554170 & -0.256129 \\
$\mathrm{Ga}$ & 4.113946 & -2.575249 & 1.925788 \\
$\mathrm{Ga}$ & 1.404986 & -2.440409 & 2.187757 \\
$\mathrm{Ga}$ & -1.367489 & -2.471596 & -2.175233 \\
$\mathrm{Ga}$ & -4.271680 & -0.749725 & 0.034915 \\
$\mathrm{Ga}$ & 4.197264 & -0.935720 & -0.217601 \\
$\mathrm{Ga}$ & 3.989054 & -2.722080 & -2.250097 \\
$\mathrm{Ga}$ & -2.502992 & -3.217626 & 0.048187 \\
$\mathrm{Ga}$ & -1.708806 & 2.132432 & 1.991208 \\
$\mathrm{Ga}$ & 1.611867 & 1.909939 & -2.483576 \\
$\mathrm{Ga}$ & 1.753088 & 2.032161 & 2.006711 \\
$\mathrm{Ga}$ & -1.723443 & 1.934361 & -2.399833 \\
$\mathrm{Ga}$ & 2.542333 & -3.239263 & -0.093518 \\
$\mathrm{Ga}$ & 0.018468 & -3.295451 & 0.002593 \\
$\mathrm{Ga}$ & -2.874072 & -0.313652 & -2.315265 \\
$\mathrm{Ga}$ & 2.871359 & -0.216799 & 2.088542 \\
$\mathrm{Ga}$ & 2.746387 & -0.354350 & -2.429966 \\
$\mathrm{Ga}$ & -2.689557 & -0.172397 & 2.304770 \\
$\mathrm{Ga}$ & 3.216691 & 1.404429 & -0.232804 \\
$\mathrm{Ga}$ & -3.217659 & 1.499694 & -0.095778 \\
$\mathrm{Ga}$ & 1.242261 & -2.537911 & -2.291203 \\
$\mathrm{Ga}$ & -1.235085 & -2.425913 & 2.252070 \\
$\mathrm{Ga}$ & 0.054676 & -0.069301 & 1.871579 \\
$\mathrm{Ga}$ & -0.057527 & -0.098324 & -2.140864 \\
$\mathrm{Ga}$ & -1.504882 & -0.730343 & -0.033238 \\
$\mathrm{Ga}$ & 1.448353 & -0.785070 & -0.118569 \\
$\mathrm{Ga}$ & 0.030323 & 1.782689 & -0.166479 \\
$\mathrm{Ga}$ & -0.029154 & 3.866087 & -2.511677 \\
$\mathrm{Ga}$ & 0.074427 & 3.997026 & 1.894865 \\
$\mathrm{Ga}$ & 0.052917 & 5.261450 & -0.327383 \\
$\mathrm{Ga}$ & -4.183144 & -2.629605 & -1.868593 \\
$\mathrm{Ga}$ & -4.001409 & -2.484058 & 2.074668 \\
& -0.066460 & 1.584592 & 4.020779 \\
\hline & & &
\end{tabular}

Table B.37. Atomic coordinates for the $\mathrm{Ga}_{34}^{+} \mathrm{GM}$ structure, as given by Ref. [46]. 
Appendix C

Basis Sets 


\begin{tabular}{crr}
\hline & Exponent & Coefficient \\
\hline \hline $\mathrm{S}$ & & \\
& 104.3829980 & 0.0031560 \\
10.9005580 & 0.0239720 \\
& 2.2685170 & -0.3310080 \\
& 0.9567350 & 1.0000000 \\
& 0.3943800 & 1.0000000 \\
& 0.1380120 & 1.0000000 \\
$\mathrm{P}$ & & \\
& & \\
& 17.9293820 & 0.0029790 \\
& 3.2048610 & -0.0600800 \\
& 1.5221960 & 0.0690590 \\
& 0.6753990 & 1.0000000 \\
& 0.2541180 & 1.0000000 \\
$\mathrm{D}$ & & \\
& & \\
& 1.046000 & 1.0000000 \\
& 0.344000 & 1.0000000 \\
\hline
\end{tabular}

Table C.1. Basis set for $\mathrm{Cl}_{2}$ DFT calculations. This basis was also used as the small, less diffuse (reference) basis set of the $\mathrm{Cl}_{2}$ bulk HF calculation in the dual basis set scheme. 


\begin{tabular}{|c|c|c|}
\hline & Exponent & Coefficient \\
\hline \multicolumn{3}{|c|}{$S$} \\
\hline & 104.3829980 & 0.0031560 \\
\hline & 10.9005580 & 0.0239720 \\
\hline & 2.2685170 & -0.3310080 \\
\hline & 0.9567350 & 1.0000000 \\
\hline & 0.3943800 & 1.0000000 \\
\hline & 0.1380120 & 1.0000000 \\
\hline & 0.0600000 & 1.0000000 \\
\hline \multicolumn{3}{|c|}{$\mathrm{P}$} \\
\hline & 17.9293820 & 0.0029790 \\
\hline & 3.2048610 & -0.0600800 \\
\hline & 1.5221960 & 0.0690590 \\
\hline & 0.6753990 & 1.0000000 \\
\hline & 0.2541180 & 1.0000000 \\
\hline & 0.0787660 & 1.0000000 \\
\hline \multicolumn{3}{|c|}{$\mathrm{D}$} \\
\hline & 1.046000 & 1.0000000 \\
\hline & 0.344000 & 1.0000000 \\
\hline & 0.135000 & 1.0000000 \\
\hline \multicolumn{3}{|c|}{$\mathrm{F}$} \\
\hline & 0.706000 & 1.0000000 \\
\hline & 0.312000 & 1.0000000 \\
\hline
\end{tabular}

Table C.2. The large, diffuse basis set used to boost the correlation energy of the HF bulk calculations in the dual basis set scheme. 


\begin{tabular}{|c|c|c|}
\hline & Exponent & Coefficient \\
\hline \multicolumn{3}{|c|}{$S$} \\
\hline & 104.3829980 & 0.0031560 \\
\hline & 10.9005580 & 0.0239720 \\
\hline & 2.2685170 & -0.3310080 \\
\hline & 0.9567350 & 1.0000000 \\
\hline & 0.3943800 & 1.0000000 \\
\hline & 0.1380120 & 1.0000000 \\
\hline & 0.0591000 & 1.0000000 \\
\hline \multicolumn{3}{|c|}{$\mathrm{P}$} \\
\hline & 17.9293820 & 0.0029790 \\
\hline & 3.2048610 & -0.0600800 \\
\hline & 1.5221960 & 0.0690590 \\
\hline & 0.6753990 & 1.0000000 \\
\hline & 0.2541180 & 1.0000000 \\
\hline & 0.0787660 & 1.0000000 \\
\hline & 0.0376000 & 1.0000000 \\
\hline \multicolumn{3}{|c|}{$\mathrm{D}$} \\
\hline & 1.046000 & 1.0000000 \\
\hline & 0.344000 & 1.0000000 \\
\hline & 0.135000 & 1.0000000 \\
\hline \multicolumn{3}{|c|}{$\mathrm{F}$} \\
\hline & 0.706000 & 1.0000000 \\
\hline & 0.312000 & 1.0000000 \\
\hline
\end{tabular}

Table C.3. Basis set for correlated molecules of the $\mathrm{Cl}_{2}$ incremental calculations. This same basis, without the $d$ - and $f$-functions, was used for the uncorrelated molecules. 


\begin{tabular}{|c|c|c|c|c|c|}
\hline & Exponent & Coefficient & & Exponent & Coefficient \\
\hline$S$ & & & $\mathrm{P}$ & & \\
\hline & 0.0901270 & 0.0897840 & & 0.0641740 & 0.0906150 \\
\hline & 0.1919290 & 0.4553640 & & 0.1442090 & 0.3433450 \\
\hline & 0.3988620 & 0.5908340 & & 0.3131070 & 0.4482900 \\
\hline & 0.9528400 & 0.2885970 & & 0.6656170 & 0.2831560 \\
\hline & 762.0066790 & 0.0000520 & & 77.0646890 & 0.0000120 \\
\hline & 376.6365900 & -0.0000230 & & 33.1900760 & 0.0001780 \\
\hline & 186.1599440 & 0.0001490 & & 14.2942400 & -0.0008620 \\
\hline & 92.0131660 & 0.0001420 & & 6.1562170 & 0.0165350 \\
\hline & 45.4792930 & 0.0001820 & & 2.6513480 & -0.1173210 \\
\hline & 22.4790230 & 0.0014490 & & 1.1418780 & -0.0129850 \\
\hline & 11.1106930 & -0.0118330 & & 0.0510520 & 1.0000000 \\
\hline & 5.4916760 & 0.1119000 & \multirow{3}{*}{$\mathrm{P}$} & & \\
\hline & 2.7143670 & -0.2561560 & & & \\
\hline & 1.3416290 & -0.4007910 & & 90 & 1.00 \\
\hline & 0.0808210 & 1.0000000 & $\mathrm{P}$ & & \\
\hline \multirow[t]{2}{*}{$S$} & & & & 0.6656170 & 1.0000000 \\
\hline & 0.1919290 & 1.0000000 & $\mathrm{D}$ & & \\
\hline \multirow{6}{*}{$S$} & \multirow{6}{*}{0.9528400} & \multirow{6}{*}{1.0000000} & & 0.6013860 & 1.0000000 \\
\hline & & & & 0.2523660 & 1.0000000 \\
\hline & & & & 0.0967350 & 1.0000000 \\
\hline & & & $\mathrm{F}$ & & \\
\hline & & & & 0.5812580 & 1.0000000 \\
\hline & & & & 0.2592890 & 1.0000000 \\
\hline
\end{tabular}

Table C.4. Basis set for correlated molecules of the $\mathrm{LC} \mathrm{Br}_{2}$ incremental calculations. This same basis, without the $d$ - and $f$-functions, was used for the uncorrelated molecules. 


\begin{tabular}{|c|c|c|c|c|c|}
\hline & Exponent & Coefficient & & Exponent & Coefficient \\
\hline \multirow[t]{16}{*}{$S$} & & & $\mathrm{P}$ & & \\
\hline & 0.0775970 & 0.1220050 & & 0.0496780 & 0.0744090 \\
\hline & 0.1656200 & 0.5526420 & & 0.1059870 & 0.3244220 \\
\hline & 0.3405190 & 0.5919630 & & 0.2183660 & 0.4617230 \\
\hline & 0.6858370 & 0.0320750 & & 0.4373980 & 0.3096330 \\
\hline & 208.3634160 & 0.0000170 & & 9.5386090 & 0.0019140 \\
\hline & 117.8734120 & 0.0000430 & & 5.9554680 & -0.0213810 \\
\hline & 66.6822490 & -0.0002200 & & 3.7183190 & 0.0942320 \\
\hline & 37.7228610 & 0.0008860 & & 2.3215470 & -0.0995310 \\
\hline & 21.3402260 & -0.0029400 & & 1.4494670 & -0.1457100 \\
\hline & 12.0723940 & 0.0131530 & & 0.9049800 & -0.0001730 \\
\hline & 6.8294820 & -0.0737860 & & 0.0418350 & 1.0000000 \\
\hline & 3.8635110 & 0.2725530 & $\mathrm{P}$ & & \\
\hline & 2.1856300 & -0.2496550 & & 0.1059870 & 1.0000000 \\
\hline & 1.2364340 & -0.4287590 & & & \\
\hline & 0.0657390 & 1.0000000 & $\mathrm{P}$ & & \\
\hline \multirow[t]{2}{*}{ S } & & & & 0.4373980 & 1.0000000 \\
\hline & 0.1656200 & 1.0000000 & $\mathrm{D}$ & & \\
\hline \multirow{9}{*}{ S } & \multirow{9}{*}{0.6858370} & & & 1.0796940 & -0.1205570 \\
\hline & & \multirow{3}{*}{1.0000000} & & 0.4488510 & 0.5583480 \\
\hline & & & & 0.1865970 & 0.5829660 \\
\hline & & & & 0.0738310 & 1.0000000 \\
\hline & & & $\mathrm{D}$ & & \\
\hline & & & & 0.1865970 & 1.0000000 \\
\hline & & & $\mathrm{F}$ & & \\
\hline & & & & 0.4293190 & 1.0000000 \\
\hline & & & & 0.1939730 & 1.0000000 \\
\hline
\end{tabular}

Table C.5. Basis set for correlated molecules of the $I_{2}$ incremental calculations. This same basis, without the $d$ - and $f$-functions, was used for the uncorrelated molecules. 


\begin{tabular}{|c|c|c|c|c|c|}
\hline & Exponent & Coefficient & & Exponent & Coefficient \\
\hline \multirow[t]{20}{*}{$S$} & & & $S$ & & \\
\hline & 9659.3900000 & 0.0003710 & & 9659.3900000 & -0.0001520 \\
\hline & 1455.9600000 & 0.0025710 & & 1455.9600000 & -0.0010080 \\
\hline & 325.9140000 & 0.0084850 & & 325.9140000 & -0.0036090 \\
\hline & 46.5657000 & 0.0782220 & & 46.5657000 & -0.0278720 \\
\hline & 18.2469000 & -0.3971490 & & 18.2469000 & 0.1513170 \\
\hline & 3.8020100 & 0.6858640 & & 3.8020100 & -0.3514970 \\
\hline & 1.7863200 & 0.4868190 & & 1.7863200 & -0.4107030 \\
\hline & 0.6027140 & 0.0349130 & & 0.6027140 & 0.3473160 \\
\hline & 0.2840140 & -0.0065730 & & 0.2840140 & 0.6754910 \\
\hline & 0.1202710 & 0.0016420 & & 0.1202710 & 0.2440080 \\
\hline & 7.0742000 & 1.0000000 & & 7.0742000 & 1.0000000 \\
\hline & 1.4954000 & 1.0000000 & & 1.4954000 & 1.0000000 \\
\hline & 0.0600000 & 1.0000000 & & 0.0600000 & 1.0000000 \\
\hline & & & $S$ & & \\
\hline & & & & 0.6027140 & 1.0000000 \\
\hline & & & $S$ & & \\
\hline & & & & 0.2840140 & 1.0000000 \\
\hline & & & $S$ & & \\
\hline & & & & 0.1202710 & 1.0000000 \\
\hline
\end{tabular}

Table C.6. Basis set for correlated molecules of the SC $\mathrm{Br}_{2}$ incremental calculations - $s$-functions. 


\begin{tabular}{|c|c|c|c|}
\hline Exponent & Coefficient & Exponent & Coefficient \\
\hline $\mathrm{P}$ & & $\mathrm{P}$ & \\
\hline 492.1870000 & 0.0004720 & 492.1870000 & -0.0001580 \\
\hline 92.3642000 & 0.0054940 & 92.3642000 & -0.0013440 \\
\hline 27.0718000 & -0.0880300 & 27.0718000 & 0.0246160 \\
\hline 7.2653900 & 0.3434850 & 7.2653900 & -0.1125320 \\
\hline 3.4848100 & 0.5102080 & 3.4848100 & -0.1797750 \\
\hline 1.6404800 & 0.2486310 & 1.6404800 & -0.0736610 \\
\hline 0.6485700 & 0.0222010 & 0.6485700 & 0.3487340 \\
\hline 0.2564070 & -0.0004190 & 0.2564070 & 0.5579480 \\
\hline 0.0953040 & 0.0006560 & 0.0953040 & 0.2664560 \\
\hline 0.7666000 & 1.0000000 & 0.7666000 & 1.0000000 \\
\hline 0.1182000 & 1.0000000 & 0.1182000 & 1.0000000 \\
\hline 10.1640000 & 1.0000000 & 10.1640000 & 1.0000000 \\
\hline 2.1057000 & 1.0000000 & 2.1057000 & 1.0000000 \\
\hline
\end{tabular}

Table C.7. Basis set for correlated molecules of the $\mathrm{Br}_{2}$ incremental calculations - $p$-functions. 


\begin{tabular}{|c|c|c|c|c|c|}
\hline & Exponent & Coefficient & & Exponent & Coefficient \\
\hline \multirow[t]{20}{*}{$\mathrm{D}$} & & & $\mathrm{D}$ & & \\
\hline & 338.9960000 & 0.0015240 & & 338.9960000 & 1.0000000 \\
\hline & 103.2170000 & 0.0156730 & & 103.2170000 & 1.0000000 \\
\hline & 42.3638000 & 0.0724000 & & 42.3638000 & 1.0000000 \\
\hline & 18.4356000 & 0.1863030 & & 18.4356000 & 1.0000000 \\
\hline & 8.3725400 & 0.3238810 & & 8.3725400 & 1.0000000 \\
\hline & 3.8022200 & 0.3745340 & & 3.8022200 & 1.0000000 \\
\hline & 1.6867700 & 0.2574180 & & 1.6867700 & 1.0000000 \\
\hline & 0.6775200 & 0.0680510 & & 0.6775200 & 1.0000000 \\
\hline & 0.2553000 & 1.0000000 & & 0.2553000 & 1.0000000 \\
\hline & 4.1557000 & 1.0000000 & & 4.1557000 & 1.0000000 \\
\hline & 1.8326000 & 1.0000000 & & 1.8326000 & 1.0000000 \\
\hline & 0.1009000 & 1.0000000 & & 0.1009000 & 1.0000000 \\
\hline & & & $\mathrm{F}$ & & \\
\hline & & & & 0.5606000 & 1.0000000 \\
\hline & & & & 8.3131000 & 1.0000000 \\
\hline & & & & 2.1829000 & 1.0000000 \\
\hline & & & & 0.2454000 & 1.0000000 \\
\hline & & & $\mathrm{G}$ & & \\
\hline & & & & 2.5366000 & 1.0000000 \\
\hline
\end{tabular}

Table C.8. Basis set for correlated molecules of the $\mathrm{Br}_{2}$ incremental calculations - $d-, f$ - and $g$-functions. The LC basis, as listed in Tab. C.4, without $d$ - and $f$-functions is used for the uncorrelated molecules. 


\section{Bibliography}

[1] P. Dirac, Proc. R. Soc. Lond. A 123, 714 (1929).

[2] K. Albe, K. Nordlund, J. Nord, and A. Kuronen, Phys. Rev. B 66, 035205 (2002).

[3] J. Shealy and J. Woodall, App. Phys. Lett. 41, 88 (1982).

[4] A. Schnepf and H. Schnockel, Angew. Chem. Int. Ed. 41, 3532 (2002).

[5] E. Voloshina, K. Rosciszewski, and B. Paulus, Phys. Rev. B 79, 045113 (2009).

[6] M. Bernasconi, G. Chiarotti, and E. Tosatti, Phys. Rev. B 52, 9988 (1995).

[7] T. Kenichi, K. Kazuaki, and A. Masao, Phys. Rev. B 58, 2482 (1998).

[8] F. Greuter and P. Oelhafen, Z. Phys. B 34, 123 (1979).

[9] S. Barman and D. Sarma, Phys. Rev. B 51, 4007 (1995).

[10] C. Søndergaard et al., Phys. Rev. B. 67, 205105 (2003).

[11] W. Reed, Phys. Rev. 188, 1184 (1969).

[12] J. Hafner and W. Jank, Phys. Rev. B 42, 11530 (1990).

[13] X. Gong, G. Chiarotti, M. Parrinello, and E. Tosatti, Phys. Rev. B 43, 14277 (1991). 
[14] S. Moré et al., Phys. Rev. B 68, 075414 (2003).

[15] D. Walko, I. Robinson, C. Grütter, and J. Bilgram, Phys. Rev. Lett. 81, 626 (1998).

[16] X. Gong, G. Chiarotti, M. Parrinello, and E. Tosatti, Europhys. Lett. 21, 469 (1993).

[17] K. Tsai, T. Wu, and S. Tsay, J. Chem. Phys. 132, 034502 (2010).

[18] J. Yang, S. John, and T. Iitaka, J. Chem. Phys. 135, 044507 (2011).

[19] L. E. González and D. J. González, Phys. Rev. B 77, 1 (2008).

[20] S. Hendy and N. Gaston, Superheating of nanoparticles, in Handbook of Nanophysics: Nanoparticles and Quantum Dots, edited by K. Sattler, pages 200-206, CRC Press Taylor and Francis, Boca Raton, FL, 2010.

[21] R. Powell, Proc. R. Soc. A 209, 525 (1951).

[22] R. W. Powell, M. J. Woodman, and R. P. Tye, Br. J. Appl. Phys. 14, 432 (1963).

[23] O. Züger and U. Dürig, Ultramicroscopy 42, 520 (1992).

[24] O. Züger and U. Dürig, Phys. Rev. B 46, 7319 (1992).

[25] G. Breaux, R. Benirschke, T. Sugai, B. Kinnear, and M. Jarrold, Phys. Rev. Lett. 91, 215508 (2003).

[26] P. Buffat and J. Borel, Phys. Rev. A 13, 2287 (1976).

[27] C. Neal, A. Starace, and M. Jarrold, Phys. Rev. B 76, 54113 (2007).

[28] A. K. Starace, B. Cao, O. H. Judd, I. Bhattacharyya, and M. F. Jarrold, J. Chem. Phys. 132, 034302 (2010).

[29] A. Shvartsburg and M. Jarrold, Phys. Rev. Lett. 85, 2530 (2000). 
[30] G. Breaux, D. Hillman, C. Neal, R. Benirschke, and M. Jarrold, J. Am. Chem. Soc. 126, 8628 (2004).

[31] G. A. Breaux, B. Cao, and M. F. Jarrold, J. Phys. Chem. B 109, 16575 (2005).

[32] S. Krishnamurty et al., Phys. Rev. B 73, 045406 (2006).

[33] M. Schmidt, R. Kusche, W. Kronmüller, B. V. Issendorff, and H. Haberland, Phys. Rev. Lett. 79, 99 (1997).

[34] G. A. Breaux, D. A. Hillman, C. M. Neal, R. C. Benirschke, and M. F. Jarrold, J. Am. Chem. Soc. 126, 8628 (2004).

[35] A. Aguado and M. Jarrold, Annu. Rev. Phys. Chem. 62, 151 (2011).

[36] M. Jarrold, J. Phys. Chem. 99, 11 (1995).

[37] C. Neal, A. Starace, and M. Jarrold, J. Am. Soc. Mass Spectrom. 18, 74 (2007).

[38] M. Daw and M. Baskes, Phys. Rev. Lett. 50, 1285 (1983).

[39] M. Daw and M. Baskes, Phys. Rev. B 29, 6443 (1984).

[40] S. Foiles, M. Baskes, and M. Daw, Phys. Rev. B 33, 7983 (1986).

[41] D. Schebarchov and S. Hendy, Phys. Rev. Lett. 96, 256101 (2006).

[42] S. Hendy and D. Schebarchov, Eur. Phys. J. D 53, 63 (2009).

[43] F. Ercolessi and J. Adams, Europhys. Lett. 26, 583 (1994).

[44] E. Noya, J. Doye, and F. Calvo, Phys. Rev. B 73, 125407 (2006).

[45] S. Chacko, K. Joshi, D. Kanhere, and S. Blundell, Phys. Rev. Lett. 92, 135506 (2004).

[46] S. Núñez, J. López, and A. Aguado, Nanoscale 4, 6481 (2012). 
[47] K. Joshi, S. Krishnamurty, and D. Kanhere, Phys. Rev. Lett. 96, 135703 (2006).

[48] B. Song and P. Cao, J. Chem. Phys. 123, 144312 (2005).

[49] N. Gaston and A. Parker, Chem. Phys. Lett. 501, 375 (2011).

[50] N. Drebov, F. Weigend, and R. Ahlrichs, J. Chem. Phys. 135, 044314 (2011).

[51] L. Sai, J. Zhao, X. Huang, and J. Wang, J. Nanosci. Nanotechnol. 12, 132 (2012).

[52] F. Calvo and F. Spiegelmann, Phys. Rev. Lett. 82, 2270 (1999).

[53] F. Calvo and F. Spiegelmann, J. Chem. Phys. 112, 2888 (2000).

[54] A. Aguado, J. Phys. Chem. B 109, 13043 (2005).

[55] A. Aguado and J. López, Phys. Rev. Lett. 94, 233401 (2005).

[56] A. Aguado, J. López, J. Alonso, and M. Stott, J. Chem. Phys. 111, 6026 (1999).

[57] A. Starace et al., J. Chem. Phys. 131, 044307 (2009).

[58] A. Starace et al., J. Chem. Phys. 129, 144702 (2008).

[59] A. Clarke and H. Jónsson, Phys. Rev. E 47, 3975 (1993).

[60] S. Hendy and B. Hall, Phys. Rev. B 64, 85425 (2001).

[61] S. Hendy and J. Doye, Phys. Rev. B 66, 235402 (2002).

[62] P. Steinhardt, D. Nelson, and M. Ronchetti, Phys. Rev. B 28, 784 (1983).

[63] J. Neirotti, F. Calvo, D. Freeman, and J. Doll, J. Chem. Phys. 112, 10340 (2000). 
[64] S. Krishnamurty, K. Joshi, S. Zorriasatein, and D. G. Kanhere, J. Chem. Phys. 127, 054308 (2007).

[65] S. Ghazi, M. Lee, and D. Kanhere, J. Chem. Phys. 128, 104701 (2008).

[66] R. Collin, Acta Crystallogr. 5, 431 (1952).

[67] B. Vonnegut and B. Warren, J. Am. Chem. Soc. 58, 2459 (1936).

[68] K. Toukan and S. Chen, Mol. Phys. 44, 693 (1981).

[69] A. Pasternak, A. Anderson, and J. Leech, J. Phys. C: Solid State Phys. 10, 3261 (1977).

[70] A. Pasternak, A. Anderson, and J. Leech, J. Phys. C: Solid State Phys. 11, 1563 (1978).

[71] Z. Gamba, E. Halac, and H. Bonadeo, J. Chem. Phys. 80, 2756 (1984).

[72] R. Lynch and H. Drickamer, J. Chem. Phys. 45, 1020 (1966).

[73] K. Takemura, S. Minomura, O. Shimomura, and Y. Fujii, Phys. Rev. Lett. 45, 1881 (1980).

[74] P. Johannsen, C. Wefringhaus, and W. Holzapfel, J. Phys. C: Solid State Phys. 20, L151 (1983).

[75] H. Fujihisa, Y. Fujii, K. Takemura, and O. Shimomura, J. Phys. Chem. Sol. 56, 1439 (1995).

[76] K. Yamasaki, J. Phys. Soc. Jap. 17 (1962).

[77] S. Nyburg, J. Chem. Phys. 40, 2493 (1964).

[78] S. Nyburg and W. Wong-Ng, Proc. R. Soc. A 367, 29 (1979).

[79] P. English and J. Leech, Chem. Phys. Lett. 23, 24 (1973).

[80] R. Wheatley and S. Price, Mol. Phys. 71, 1381 (1990). 
[81] K. Mukose, R. Fukano, H. Miyagi, and K. Yamaguchi, J. Phys.: Condens. Matter 14, 10441 (2002).

[82] P. Li, G. Gao, and Y. Ma, J. Chem. Phys. 137, 064502 (2012).

[83] D. Williams and D. Gao, Inorg. Chem. 36, 782 (1997).

[84] D. Duan et al., Phys. Rev. B 76, 104113 (2007).

[85] M. Born and R. Oppenheimer, Ann. Phys. 389, 457 (1927).

[86] A. Szabo and N. S. Ostlund, Modern Quantum Chemistry: Introduction to Advanced Electronic Structure Theory, (Dover Publications, Inc., Mineola, New York, 1996).

[87] P. Echenique and J. Alonso, Mol. Phys. 105, 3057 (2007).

[88] M. Born and V. Fock, Z. Phys. A 51, 165 (1928).

[89] T. Kato, J. Phys. Soc. Jpn. 5, 435 (1950).

[90] D. Hartree, Proc. Cambridge Philos. Soc. 24, 89 (1928).

[91] V. Fock, Z. Phys. A 61, 126 (1930).

[92] J. Slater, Phys. Rev. 35, 210 (1930).

[93] J. O. Trygve Helgaker, Poul Jørgensen, Molecular Electronic-Structure Theory, (John Wiley and Sons, Ltd., Chichester, West Sussex, 2002).

[94] M. Schütz and F. Manby, Phys. Chem. Chem. Phys. 5, 3349 (2003).

[95] S. Saebo and P. Pulay, Ann. Rev. Phys. Chem. 44, 213 (1993).

[96] L. Thomas, Math. Proc. Cambridge Philos. Soc 23, 542 (1927).

[97] E. Fermi, Z. Physik. 48, 73 (1928). 
[98] R. O. Jones, Introduction to density functional theory and exchangecorrelation energy functionals, in Computational Nanoscience: Do It Yourself!, edited by D. M. J. Grotendorst, S. Blügel, Jülich, Germany, 2006, 31, 45-70.

[99] D. Marx and J. Hutter, Ab Initio Molecular Dynamics: Basic Theory and Advanced Methods, (Cambridge University Press, New York, New York, 2009), pp. 68-72.

[100] P. Hohenberg and W. Kohn, Phys. Rev. 136, B864 (1964).

[101] W. Kohn and L. Sham, Phys. Rev. 140, A1133 (1965).

[102] L. Sham and W. Kohn, Phys. Rev. 145, 561 (1966).

[103] S. Vosko, L. Wilk, and M. Nusair, Can. J. Phys. 58, 1200 (1980).

[104] J. Perdew and Y. Wang, Phys. Rev. B 45, 13244 (1992).

[105] J. Perdew, K. Burke, and M. Ernzerhof, Phys. Rev. Lett. 77, 3865 (1996).

[106] A. Becke, Phys. Rev. A 38, 3098 (1988).

[107] C. Lee, W. Yang, and R. Parr, Phys. Rev. B 37, 785 (1988).

[108] A. Becke, J. Chem. Phys. 98, 5648 (1993).

[109] R. Jones and O. Gunnarsson, Rev. Mod. Phys. 61, 689 (1989).

[110] E. Engel, A. Hock, and R. Dreizler, Phys. Rev. A 61, 32502 (2000).

[111] N. Gaston and P. Schwerdtfeger, Phys. Rev. B 74, 024105 (2006).

[112] N. Gaston, B. Paulus, K. Rosciszewski, P. Schwerdtfeger, and H. Stoll, Phys. Rev. B 74, 094102 (2006).

[113] G. Kresse and J. Hafner, Phys. Rev. B 47, 558 (1993). 
[114] G. Kresse and J. Hafner, Phys. Rev. B 49, 14251 (1994).

[115] G. Kresse and J. Furthmüller, Comp. Mat. Sci. 6, 15 (1996).

[116] G. Kresse and J. Furthmüller, Phys. Rev. B 54, 11169 (1996).

[117] H. Hellman, Einführung in die Quantenchemie, (Deuticke, Wien, 1937).

[118] R. Feynman, Phys. Rev. 56, 340 (1939).

[119] L. Verlet, Phys. Rev. 165, 201 (1968).

[120] H. Stoll, J. Chem. Phys. 97, 8449 (1992).

[121] H. Stoll, Chem. Phys. Lett. 191, 548 (1992).

[122] H. Stoll, Phys. Rev. B 46, 6700 (1992).

[123] B. Paulus, Phys. Rep. 428, 1 (2006).

[124] C. Müller and B. Paulus, Phys. Chem. Chem. Phys. 14, 7605 (2012).

[125] M. Valiev et al., Comp. Phys. Comm. 181, 1477 (2010).

[126] A. Wilson, D. Woon, K. Peterson, and T. Dunning, J. Chem. Phys. 110, 7667 (1999).

[127] J. Perdew et al., Phys. Rev. B 46, 6671 (1992).

[128] D. Schebarchov and N. Gaston, Phys. Chem. Chem. Phys. 14, 9912 (2012).

[129] N. Drebov and R. Ahlrichs, J. Chem. Phys. 132, 164703 (2010).

[130] P. Blöchl, Phys. Rev. B 50, 17953 (1994).

[131] G. Kresse and D. Joubert, Phys. Rev. B 59, 1758 (1999).

[132] J. Perdew et al., Phys. Rev. B 48, 4978 (1993). 
[133] http://lammps.sandia.gov.

[134] S. Plimpton, J. Comp. Phys. 117, 1 (1995).

[135] W. Hoover, Phys. Rev. A 31, 1695 (1985).

[136] J. Tersoff, Phys. Rev. B 39, 5566 (1989).

[137] J. Tersoff, Phys. Rev. Lett. 61, 2879 (1988).

[138] S. Nosé, J. Chem. Phys. 81, 511 (1984).

[139] G. J. Martyna, G. J. Martyna, and M. Tuckerman, J. Chem. Phys. 97, 2635 (1992).

[140] F. Calvo, J. Galindez, and F. Gadéa, J. Phys. Chem. A 106, 4145 (2002).

[141] A. Vichare, D. Kanhere, and S. Blundell, Phys. Rev. B 64, 045408 (2001).

[142] F. Calvo, J. Neirotti, D. Freeman, and J. Doll, J. Chem. Phys. 112, 10350 (2000).

[143] N. Metropolis, A. Rosenbluth, M. Rosenbluth, and A. Teller, J. Chem. Phys. (1953).

[144] T. Beck and R. Berry, J. Chem. Phys. 88, 3910 (1988).

[145] U. Röthlisberger and W. Andreoni, J. Chem. Phys. 94, 8129 (1991).

[146] R. Poteau, F. Spiegelmann, and P. Labastie, Z. Phys. D 30, 57 (1994).

[147] W. Curtin and N. Ashcroft, Phys. Rev. Lett. 56, 2775 (1986).

[148] R. Bader., Atoms in Molecules: a quantum theory, International series of monographs on chemistry, volume 22, Clarendon Press, Oxford, UK, 1990. 
[149] D. Schebarchov and N. Gaston, Phys. Chem. Chem. Phys. 13, 21109 (2011).

[150] M. Cohen, M. Chou, W. Knight, and W. de Heer, J. Phys. Chem. 91, 3141 (1987).

[151] M. Brack, Rev. Mod. Phys. 65, 677 (1993).

[152] A. Becke and K. Edgecombe, J. Chem. Phys. 92, 5397 (1990).

[153] B. Silvi and A. Savin, Nature 371, 683 (1994).

[154] A. Savin, R. Nesper, S. Wengert, and T. Fässler, Angew. Chem., Int. Ed. Engl. 36, 1808 (1997).

[155] J. Jellinek and A. Goldberg, J. Chem. Phys. 113, 2570 (2000).

[156] C. Bichara, J. Gaspard, and J. Mathieu, Phys. Lett. A 119, 462 (1987).

[157] A. Ferrenberg and R. Swendsen, Phys. Rev. Lett. 61, 2635 (1988).

[158] P. Labastie and R. Whetten, Phys. Rev. Lett. 65, 1567 (1990).

[159] F. Calvo and P. Labastie, Chem. Phys. Lett. 247, 395 (1995).

[160] P. Blaise and S. Blundell, Phys. Rev. B 63, 235409 (2001).

[161] K. Steenbergen, D. Schebarchov, and N. Gaston, J. Chem. Phys. 137, 144307 (2012).

[162] A. Aguado and J. López, J. Chem. Phys. 130, 064704 (2009).

[163] M. Schmidt, R. Kusche, B. von Issendorff, and H. Haberland, Nature 393, 21 (1998).

[164] M. Haruta, N. Yamada, T. Kobayashi, and S. Iijima, J. Catal. 115, 301 (1989).

[165] M. Haruta, Chem. Rec. 3, 75 (2003). 
[166] B. Cao, A. Starace, O. Judd, and M. Jarrold, J. Am. Chem. Soc. 131, 2446 (2009).

[167] F. Baletto and R. Ferrando, Rev. Mod. Phys. 77, 371 (2005).

[168] B. Assadollahzadeh, S. Schäfer, and P. Schwerdtfeger, J. Comp. Chem. 31, 929 (2010).

[169] F. Chuang, C. Wang, and K. Ho, Phys. Rev. B 73, 125431 (2006).

[170] D. Wales and R. Berry, J. Chem. Phys. 92, 4283 (1990).

[171] T. Beck, D. Leitner, and R. Berry, J. Chem. Phys. 89, 1681 (1988).

[172] I. Garzón and J. Jellinek, Z. Phys. D 20, 235 (1991).

[173] I. Garzón, I. Kaplan, R. Santamaria, and O. Novaro, J. Chem. Phys. 109, 2176 (1998).

[174] V. Bonačić-Koutecký, J. Jellinek, M. Wiechert, and P. Fantucci, J. Chem. Phys. 107, 6321 (1997).

[175] K. Joshi, D. Kanhere, and S. Blundell, Phys. Rev. B 66, 155329 (2002).

[176] R. Werner, Eur. Phys. J. B 43, 47 (2005).

[177] M. Schmidt, J. Donges, T. Hippler, and H. Haberland, Phys. Rev. Lett. 90, 103401 (2003).

[178] H.-J. Werner et al., Molpro, version 2010.1, a package of ab initio programs, 2010, see http://www.molpro.net.

[179] R. Dovesi et al., Z. Kristallogr. 220, 571 (2005).

[180] R. Dovesi et al., CRYSTAL09 User's Manual, (University of Torino, Torino, 2009).

[181] C. Pisani et al., J. Comp. Chem. 29, 2113 (2008). 
[182] A. Erba and M. Halo, CRYSCOR09 User's Manual, (University of Torino, Torino, 2009, www.cryscor.unito.it).

[183] C. Adamo and V. Barone, J. Chem. Phys. 110, 6158 (1999).

[184] S. Grimme, J. Comp. Chem. 27, 1787 (2006).

[185] A. Bergner, M. Dolg, W. Küchle, H. Stoll, and H. Preuß, Mol. Phys. 80, 1431 (1993).

[186] D. Woon and T. Dunning, J. Chem. Phys. 100, 2975 (1994).

[187] B. Paulus, K. Rosciszewski, N. Gaston, P. Schwerdtfeger, and H. Stoll, Phys. Rev. B 70, 165106 (2004).

[188] B. Powell, K. Heal, and B. Torrie, Mol. Phys. 53, 929 (1984).

[189] P. Harris, E. Mack, and F. Blake, J. Am. Chem. Soc. 50, 1583 (1928).

[190] B. Sharma and J. Donohue, Z. Krist. 117, 293 (1962).

[191] K. Peterson, D. Figgen, E. Goll, H. Stoll, and M. Dolg, J. Chem. Phys. 119, 11113 (2003).

[192] K. A. Peterson and K. E. Yousaf, J. Chem. Phys. 133, 174116 (2010).

[193] J. Boughton and P. Pulay, J. Comp. Chem. 14, 736 (1993).

[194] C. Hampel and H. Werner, J. Chem. Phys. 104, 6286 (1996).

[195] L. Hsu and D. Williams, Inorg. Chem. 18, 79 (1979).

[196] K. Kobashi and R. Etters, J. Chem. Phys. 79, 3018 (1983). 